\title{
ale Kooperation
}

\section{$12156 a$}

Eberhard Spittler

Funktionsanalyse, Wirksamkeit und Alternativen der Sysmin-Regelung in den AKP-EWG-Abkommen von Lomé

Nomos Verlagsgesellschaft

Baden-Baden 
Funktionsanalyse, Wirksamkeit und Alternativen der Sysmin-Regelung in den AKP-EWG-Abkommen von Lomé

Von der Fakultät für Wirtschaftswissenschaften der Rheinisch-Westfälischen Technischen Hochschule Aachen zur Erlangung des akademischen Grades eines Doktors der Wirtschafts- und Sozialwissenschaften genehmigte Dissertation

\author{
vorgelegt von \\ Diplom-Geophysiker \\ Eberhard Spittler \\ aus Frankfurt am Main
}

Referent: Universitätsprofessor Dr. Dr. Werner Gocht Korreferent: Universitätsprofessor Dr. Klaus Dorner

Tag der mündlichen Prüfung: 6. Juli 1993 


\section{Internationale Kooperation}

Aachener Studien zur internationalen technischwirtschaftlichen Zusammenarbeit

Eine Schriftenreihe des Forschungsinstituts für Internationale Technische und Wirtschaftliche Zusammenarbeit der Rheinisch-Westfälischen Technischen Hochschule Aachen

Herausgegeben von Werner Gocht und Hubertus Seifert Band 41 
Se 1218 ra

Eberhard Spittler

Funktionsanalyse, Wirksamkeit und Alternativen der SysminRegelung in den AKP-EWGAbkommen von Lomé

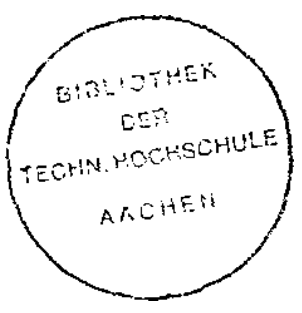


Schriftleitung: Hubertus Seifert, Aachen

Die Deutsche Bibliothek - CIP-Einheitsaufnahme

Spittler, Eberhard:

Funktionsanalyse, Wirksamkeit und Alternativen der Sysmin-Regelung in den AKP-EWG-Abkommen von Lomé / Eberhard Spittler. - 1. Aufl, - Baden-Baden: Nomos Verl.-Ges., 1994

(Internationale Kooperation; Bd. 41)

Zugl.: Aachen, Techn. Hochsch., Diss., 1993

ISBN 3-7890-3319-7

NE: GT

D 82 (Diss. T.H. Aachen)

1. Auflage 1994

(c) Nomos Verlagsgesellschaft, Baden-Baden 1994. Printed in Germany. Alle Rechte, auch die des Nachdrucks von Auszügen, der photomechanischen Wiedergabe und der Übersetzung, vorbehalten. 


\section{VORWORT DER HERAUSGEBER}

Die bisherige Entwicklungspolitik der Europäischen Gemeinschaft steht seit der Entscheidung des Europäischen Rates über den Maastricht-Vertrag im Dezember 1991 auf dem Prüfstand. Es gilt, das eher unkoordinierte Nebeneinander von Entwicklungshilfe der 12 Mitgliedsstaaten und der EG-Kommission zu einem einheitlichen Konzept mit adäquater Arbeitsteilung auf Gemeinschafts- und Länderebene zu schmieden.

Die Rohstoffpolitik war seit der 1. Lome-Konvention ein Kernstück der Zusammenarbeit mit den AKP-Ländern. Verschiedene Instrumente der Rohstoffpolitik wurden getestet und verändert, wobei die Stabilisierung von Rohstofferlösen der Entwicklungsländer als herausragendes Ziel definiert wird. Das Erlösstabilisierungssystem STABEX und das äbnliche Bergbauunterstützungssystem SYSMIN gehören zu den bemerkenswerten Instrumenten der EG-Entwicklungspolitik, die aber im Laufe der Jahre immer stärkerer Kritik ausgesetzt waren, weil deutliche Schwächen in der Mittelverwendung und in der administrativen Abwicklung auftraten.

Es war deshalb das verdienstvolle Anliegen der Arbeit von Herrn Spittler, eine umfassende Analyse von SYSMIN durchzuführen und vielfältige Anregungen für Verbesserungen aufzuzeigen. Die vorliegende Studie beweist dann auch, da $B$ durch verschiedene Modifikationen des SYSMIN die Effizienz gesteigert werden könnte und SYSMIN nicht notwendigerweise ganz aufgegeben werden muß. 


\section{VORWORT DES VERFASSERS}

Die Entstehung der vorliegenden Arbeit, deren Thema unterschiedliche Facetten der internationalen Zusammenarbeit berührt, wurde von vielen Personen begleitet.

Ganz besonderer Dank gilt Herrn Professor Dr. Dr. Werner Gocht, der das Thema der Arbeit formulierte, die empirischen Erhebungen in Sambia initiierte und die Arbeit in Form von Anregungen, Hinweisen und moralischer Unterstützung betreute.

Herrn Professor Dr. Klaus Dorner ist zu danken für die Übernahme des Korreferates und die konstruktiven Anregungen zur Abrundung der Arbeit.

Herr Dr. E. Pluhar, Ziraco Sambia hat den Aufenthalt in Sambia arrangiert und die Kontakte zur Zambia Consolidated Copper Mines hergestellt. Herr Dr. K. Kolokko hat in großzügiger Weise die Begehung vieler Abbau- und Hüttenbetriebe im Kupfergürtel Sambias ermöglicht und einen ungehinderten Informationstransfer zugelassen.

Informationen von europäischer Seite wurden im wesentlichen von Herrn Van Den Heuvel, EG-Kommission, Brüssel sowie Herrn Walendy, Bundesministerium für Wirtschaft, Bonn beigetragen; allen dafür meinen Dank.

Sorgfältig und rasch wurde die Arbeit und ihre Modifikationen von Frau Uschi Rüttener und Frau Irene Siebcke geschrieben; ihnen sei hier ebenso herzlich gedankt wie Herrn Peter Fix, der die redaktionelle Überarbeitung übernommen hat. 


\section{INHALTSVERZEICHNIS}

Seite

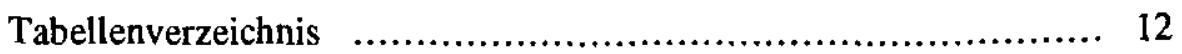

Abbildungsverzeichnis ........................................... 15

Kartenverzeichnis ................................................... 16

Abkürzungsverzeichnis ............................................ 17

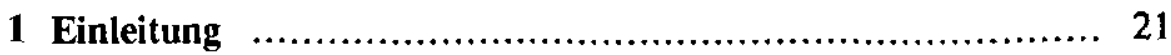

1.1 Problemstellung und Zielsetzungen $\ldots \ldots \ldots \ldots \ldots \ldots \ldots \ldots \ldots . \ldots \ldots \ldots$

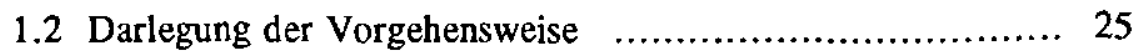

1.3 Bisherige Forschungsansätze ............................. 27

2 Die AKP-EWG-Abkommen von Lomé $\ldots \ldots \ldots \ldots \ldots \ldots \ldots \ldots . \ldots \ldots \ldots \ldots$

2.1 Darstellung von Theorien zur Erklärung von Unterentwicklung sowie von Strategien zur Ubberwindung von Unterentwicklung

2.1.1 Theorien zur Erklärung von Unterentwicklung (Entwicklungstheorien)

2.1.2 Strategien zur Überwindung von Unterentwicklung (Entwicklungsstrategien)

2.2 Die Grundsätze der Entwicklungspolitik der Europäischen Gemeinschaft 
Seite

2.3 Die Entstehung, Zielsetzungen und Konzepte der Lomé-Abkommen

2.4 Die Organe der Lomé-Abkommen .......................... 64

2.5 Die Instrumente der Lomé-Abkommen ....................... 65

2.5.1 Die handelspolitischen Instrumente $\ldots \ldots \ldots \ldots \ldots \ldots \ldots 66$

2.5.2 Stabilisierung der Erlöse aus der Ausfuhr von landwirtschaftlichen Grundstoffen (Stabex) ..........6696

2.5.3 Besondere Finanzierungsfazilität für Bergbauerzeugnisse (Sysmin)

2.5.4 Sonstige Instrumente der Lomé-Abkommen $\ldots \ldots \ldots . . .86$

2.5.5 Funktionelle Abgrenzung von Stabex und Sysmin .... 94

2.6 Anmerkungen zum Europäischen Entwicklungsfonds (EEF) und der Europäischen Recheneinheit (ECU)

2.7 Weiterführende Aspekte der besonderen Finanzierungsfazilität für Bergbauerzeugnisse (Sysmin) $\ldots \ldots \ldots \ldots \ldots \ldots . . . . .101$

2.8 Diskussion und kritische Würdigung der Lome-Abkommen 108 
Seite

3 Mineralrohstoffwirtschaftliche Aspekte der Zusammenarbeit AKP-EG

3.1 Die Versorgung der Europäischen Gemeinschaft mit mineralischen Rohstoffen

3.2 Das Bergbaupotential und die Mineralrohstoffproduktion der AKP-Staaten

3.3 Der Rohstoffhandel zwischen der Europäischen Gemeinschaft und den AKP-Staaten

3.4 Die Situation auf den Märkten der Sysmin-Rohstoffe in den siebziger und achtziger Jahren

4 Die Stärken und Schwächen der Sysmin-Regelung

4.1 Die Durchführbarkeitsbestimmungen der SysminRegelung

4.2 Die Leistungen unter der Sysmin-Regelung 164

4.3 Die Analyse der Durchführbarkeit der Sysmin-Regelung anhand von zwei Fallbeispielen

4.3.1 Fallbeispiel Sambia 168

4.3.2 Fallbeispiel Simbabwe 185

4.4 Die Möglichkeiten der Sysmin-Regelung zur Schaffung von Verknüpfungs- und Sekundäreffekten

4.5 Die Einflüsse der Sysmin-Regelung auf die Exportstruktur von AKP-Bergbaustaaten 
Seite

4.6 Die Signifikanz der Sysmin-Regelung für den rohstoffpolitischen Bereich der Europäischen Gemeinschaft

5 Die Sysmin-Regelung als entwicklungspolitisches Instrument

5.1 Die Ansätze der Sysmin-Regelung zur Umsetzung von Strategien zur Überwindung von Unterentwicklung (Entwicklungsstrategien)

5.2 Die Signifikanz der Sysmin-Regelung für den entwicklungspolitischen Bereich

6 Die Möglichkeiten zur Verbesserung der Sysmin-Regelung in den Lomé-Abkommen

6.1 Ergänzungen zur Sysmin-Regelung

6.2 Modifikationen der Sysmin-Schwellen

6.3 Die Sysmin-Erweiterung auf Produkte aus der Weiterverarbeitung

6.4 Die Modifikation der Zahlungsmodalitäten 243

6.5 Ansatzpunkte für weitere Maßnahmen der Europäischen Gemeinschaft auf dem AKP-Bergbausektor 253

6.6 Ansatzpunkte für eine Erweiterung des Sysmin-Verfahrens über das Lomé-Abkommen hinaus 
Seite

7 Zusammenfassung

Summary 269

Anhang 1: Wichtige Eckdaten der AKP-Staaten 271

Anhang 2: Die Bedeutung des Bergbausektors für einige AKP-Staaten

Anhang 3: Die Verwendung der Sysmin-Rohstoffe in den

EG-Industrien 283

Anhang 4: Die Sysmin-Regelung des Zweiten, Dritten und Vierten AKP-EWG-Abkommens von Lomé

Anhang 5: Gegenüberstellung der Sysmin-Artikel des Lomé-II-, Lomé-III- und Lomé-IV-Abkommens 309

Literaturverzeichnis 


\section{TABELLENVERZEICHINIS}

Seite

Tabelle 1: Die finanziellen Aufwendungen der Europäischen

Gemeinschaft zugunsten der AKP-Staaten

(in Mio. ECU)

Tabelle 2: Stabex-Transfer für Eisenerze während des Lomé-I-

Abkommens (in Mio. ECU)

Tabelle 3: Eisenerz-Produktion und -Export nach der EG der vier AKP-Staaten, die für den Bergbausektor

Stabex-Darlehen erhalten haben

Tabelle 4: Lomé-III-Abkommen: Finanzielle Mittel, die zugunsten des Bergbau-Sektors eingesetzt werden konnten

Tabelle 5: Die Verteilung und Förderung mineralischer Rohstoffe im Vergleich mit dem finanziellen Engagement europäischer Bergbauunternehmen

Tabelle 6: Finanzierungen der Europäischen Investitionsbank im AKP-Mineralrohstoffbereich in den Jahren 1981,1983 und 1989

Tabelle 7: Beiträge der EG-Mitgliedstaaten zu den Europäischen Entwicklungsfonds (EEF)

Tabelle 8: Importanteil der Europäischen Gemeinschaft an mineralischen Rohstoffen bezogen auf die Gesamtheit der Industrieländer (in Prozent)

Tabelle 9: Importe mineralischer Rohstoffe nach der Europäischen Gemeinschaft 1985 und deren Anteil am Gesamtbedarf 
Seite

Tabelle 10: Die Sysmin-Bergbauprodukte und mineralischen Rohstoffe mit hoher EG-Importrate (=85 \%) nach Herkunft 1982 (in Prozent)

Tabelle 11: Der Rang wichtiger AKP-Staaten unter den Bergbauproduzenten im Jahre 1985

Tabelle 12: Die Lieferungen von Bergbauerzeugnissen (außer Energierohstoffen und Edelmetallen) der AKP-Staaten nach der Europäischen Gemeinschaft 1980

Tabelle 13: AKP-Staaten als Mitglieder von Rohstoffvereinbarungen

Tabelle 14: Die Unterschiede zwischen mineralischen und agrarischen Rohstoffen

Tabelle 15: Rohstoffpreisindizes für wesentliche AKP-Rohstoffe (Basis $1980=100$ )

Tabelle 16: Die größten AKP-Lieferanten von Sysmin-Bergbauprodukten nach der Europäischen Gemeinschaft in den Jahren 1975, 1980 und 1983

Tabelle 17: Sambias Leistungsbilanz für die Jahre 1987 und 1988 (in Mio. Kwacha)

Tabelle 18: Die Zusammensetzung des Bruttoinlandsproduktes von Sambia (in Prozent) 176

Tabelle 19: Die Bergbauproduktion der ZCCM 177

Tabelle 20: Abnehmerstaaten der ZCCM-Kupferproduktion 178

Tabelle 21: Die Bergbauproduktion Simbabwes 189 
Seite

Tabelle 22: Sysmin-Rohstoffe nach der EG aus AKP-Staaten, deren Exporterlöse für diese Produkte unter der erforderlichen Abhängigkeitsschwelle blieben

Tabelle 23: Konditionen und Auswirkungen der Sysmin-Regelung auf das Bergbauunternehmen und den AKP-Staat ....... 224

Tabelle 24: Modell für variable Schuldendienstleistungen an den Europäischen Entwicklungsfonds (EEF) 


\section{ABBILDUNGSVERZEICHNIS}

Seite

Abbildung 1: Die unterschiedlichen Zielvorstellungen der LoméVertragspartner auf dem Bergbausektor

Abbildung 2: Die Qualifizierung der unterschiedlichen Interessen an den Lomé-Abkommen .......................... 59

Abbildung 3: Der Handel zwischen EG, AKP-Staaten und übriger Welt 1981 und 1991

Abbildung 4: Der EG-AKP-Handel 1988, gesamt sowie einige SITC-Warenklassen

Abbildung 5: Eisenerz-Exporte Liberias und Mauretaniens nach der EG sowie Stabex-Darlehen, die durch Exporterlösrückgänge der Eisenerze ausgelöst wurden

Abbildung 6: Die Auslösebedingungen des Sysmin-Verfahrens nach Artikel 215 des Lomé-IV-Abkommens

Abbildung 7: Zusammengefaßte Rohstoffpreisindizes $(1970$ - 1987)

Abbildung 8: Die Preisentwicklung der Sysmin-Rohstoffe (Basis $1973=100$ ) 138

Abbildung 9a-g: Die Produktions- und Preisentwicklung der Sysmin-Rohstoffe (1973 - 1989) 140

Abbildung 10: Anknüpfungspunkte und Auswirkungen der Sysmin-Regelung in Form von Multiplikatoreffekten auf die beteiligten Volkswirtschaften 


\section{KARTENVERZEICHNIS}

Seite

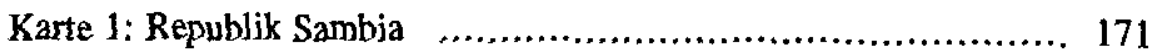

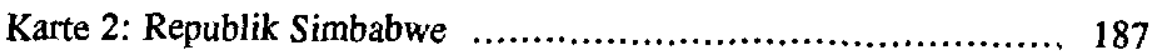




\section{ABKÜRZUNGSVERZEICHNIS}

a

AASM Assoziation Afrikanischer Staaten und Madagaskar

Abs.

Abschn.

ADB

AKP-Staaten Entwicklungsländer des afrikanischen, karibischen und pazifischen Raumes, Mitunterzeichner der LoméAbkommen (s. Anhang 1)

$\mathrm{Al}$

APEF

Art.

ATPC

$\mathrm{Au}$

ASEAN

BfAI

BIP

BMWi

BMZ

BPL

Jahr/Jahre

Absatz

Abschnitt

African Development Bank

Aluminium

Association des Pays Exportateurs de Minerai de Fer

Artikel

Association of Tin Producing Countries

Gold

Association of South East Asian Nations

Bundesstelle für Außenhandelsinformation

Bruttoinlandsprodukt

Bundeswirtschaftsministerium, Bonn

Bundesministerium für wirtschaftliche Zusammenarbeit, Bonn

bone phosphate of lime 

BSP
Bruttosozialprodukt
CIMNF Centre d'Information de Métaux Non Ferreux
CIPEC Conseil Intergouvernemental de Pays Exportateurs de Cuivere
Co
Kobalt
Comex
New York Commodity Exchange
$\mathrm{Cr}$
Chrom
$\mathrm{Cu}$
Kupfer
DAC
Development Assistance Committee
DIW
Deutsches Institut für Wirtschaftsforschung
DM
Deutsche Mark
ECU
European Currency Unit
EDF
European Development Bank
EEF
Europäischer Entwicklungsfonds
EG
Europäische Gemeinschaften
EIB
Europäische Investitionsbank, Luxemburg
ERE
Europäische Rechnungseinheit (seit 1975)
EWG
Europäische Wirtschaftsgemeinschaft
FAZ
Frankfurter Allgemeine Zeitung
$\mathrm{Fe}$
Eisen
GBP
Britisches Pfund 
GRIP Guaranteed Recovery Investment Principal

IBRD International Bank for Reconstruction and Development; Organisation der Weltbankgruppe

EFC International Finance Corporation

IMF International Monetary Fund

ITA International Tin Agreement

ite Institut zur Erforschung technologischer Entwicklungslinien, Hamburg

i.w. im wesentlichen

k.A. keine Angaben

KLCE Kuala Lumpur Commodity Exchange

KWS Kohlenwasserstoffe

LDC Least Developed Country

LME London Metal Exchange

Mn Mangan

Mo Molybdän

$\mathrm{Nb} \quad \mathrm{Niob}$

Nimexe Warenverzeichnis für die Statistik des Außenhandels der Europäischen Gemeinschaft und des Handels zwischen den Mitgliedstaaten

OECD Organisation for Economic Cooperation and Development

o.J. ohne Jahresangabe

o.O. ohne Ortsangabe 


$\begin{array}{ll}\text { o.V. } & \text { ohne Verfasserangabe } \\ \mathrm{P}_{2} \mathrm{O}_{5} & \text { Phosphate } \\ \text { PTA } & \text { Primary Tungsten Association } \\ \text { RE } & \text { Rechnungseinheit der EWG (bis 1975) } \\ \text { SITC } & \text { Internationales Warenverzeichnis für den Außenhandel } \\ \text { Sn } & \text { Zinn }\end{array}$

Stabex System zur Stabilisierung der Erlöse aus der Ausfuhr von landwirtschaftlichen Grundstoffen

Sysmin Système Minière; Besondere Finanzierungsfazilität für Bergbauerzeugnisse

$\mathrm{t}$ metrische Tonne

Ta Tantal

Ti Titan

U Uran

UI Uranium Institute

ÜLG Überseeische Länder und Gebiete (einzelner EG-Staaten)

UN(O) United Nations (Organization)

UNCTAD United Nations Conference on Trade and Development

UNCED United Nations Conference on Environment and Development

US-\$ US-Dollar

V Vanadium 
VWD/NfA Vereinigter Wirtschaftsdienst/Nachrichten für den Außenhandel

$\mathrm{Zr}$

Zirkon

ZCCM

Zambia Consolidated Copper Mines

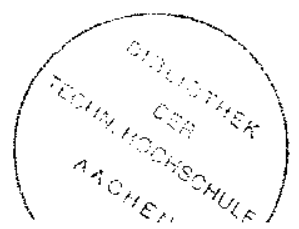





\section{EINLEITUNG}

\subsection{Problemstellung und Zielsetzung}

Der rasante politische Wandel in den ehemaligen osteuropäischen Staatshandelsländern zu Beginn der neunziger Jahre sowie die jüngsten Erkenntnisse im Umweltbereich haben der Diskussion über die Beziehungen der Industrieländer zur Dritten Welt neue Nahrung gegeben.

In den letzten Jahrzehnten fühlten sich die Industrieländer den Entwicklungsländern gegenüber häufig enger verbunden beziehungsweise stärker verpflichtet als gegenüber den Ländern des Ostblocks. Der Bezug von Rohstoffen, der Export von Investitionsgütern sowie das seit Mitte der achtziger Jahre gemeinsame Bemühen um eine Lösung der Schuldenkrise führte zu engeren Beziehungen zwischen den Industrieländern und einem Teil der Entwicklungsländer als zwischen den Industrieländern und dem größten Teil der Staatshandelsländer.

Durch den in den meisten osteuropäischen Staatshandelsländern unerwartet raschen Zerfall der bestehenden Strukturen Ende der achtziger und zu Beginn der neunziger Jahre sowie die Bereitschaft der Industrieländer im ehemaligen Ostblock unterstützend aktiv zu werden ${ }^{1}$, trifft diese Tatsache nicht mehr uneingeschränkt zu.

Die aufgrund der Größenordnung der in den ehemaligen Staatshandelsländern erforderlichen Maßnahmen ${ }^{2}$ geäußerte Sorge der Entwicklungsländer, sie könnten durch die Industrieländer nunmehr weniger Unterstützung bei ihrer Entwicklungsarbeit erfahren, ist nicht unberechtigt. Zusätzlich verstärkt wird diese Befürchtung durch die Bemühungen der Europäischen Gemeinschaft, nach dem Jahre 1992 einen Europäischen Binnenmarkt zu etablieren. ${ }^{3}$

1 Zum Beispiel durch Gründung der Europäischen Bank für Wiederaufbau und Entwicklung im April 1991 in London, vgl.: O.V. EuropaBank wird eröffnet, in: Frankfurter Allgemeine Zeitung, 13. April 1991.

2 o.V.: Umfangreiche EG-Hilfe für Osteuropa, in: Blick durch die Wirtschaft, 12. Dezember 1990.

3 Vgl. O. V.: Europas Pflichten für den Rest der Welt, in: Europa ohne Grenzen, Alarm für die Umwelt, Spiegel Spezial, 1/1992, S. 152 f. 
Gleichzeitig tritt im Rahmen der Diskussion um die globale Umweltkrise die Beziehung zwischen den Industriestaaten und der Dritten Welt in eine neue Dimension. Auf dem im Juni 1992 in Rio de Janeiro abgehaltenen Umweltgipfel (UNCED) wurde deutlich, daß nur ein gemeinsames Herangehen an die Umweltprobleme eine Möglichkeit zur Lösung der anstehenden Aufgaben bietet. ${ }^{4}$

Insofern erscheint es in dieser Phase der Umorientierung und Neugewichtung der internationalen Beziehungen mehr als angebracht zu sein, die bisherigen Wege der Zusammenarbeit aufzuzeigen, kritisch zu bewerten und Vorschläge für eine Optimierung und Effizienzsteigerung zu unterbreiten.

Entwicklungshilfe, Nord-Süd-Dialog, internationale Zusammenarbeit und Schuldenkrise - diese Schlagworte standen für eine Veränderung, die während der letzten Jahrzehnte in den Beziehungen zwischen den Industrienationen und den Entwicklungsländern stattgefunden hatte.

Besonders seit der ersten UN-Konferenz für Handel und Entwicklung (UNCTAD) im Jahre 1964 in Genf sind die Bemühungen intensiviert worden, um neue Formen der Zusammenarbeit zu finden. Kein Industrieland selbst wenn es sich nicht um eine ehemalige Kolonialmacht handelte konnte und wollte sich dieser Entwicklung entziehen. Dies trifft ebenfalls für die Organisationen und Institutionen mit internationalem oder supranationalem Charakter zu, darunter auch für die Europäische Gemeinschaft.

Die Europäische Gemeinschaft machte den Anfang mit Abkommen zwischen der Europäischen Wirtschaftsgemeinschaft und Entwicklungsländern, zu denen besondere historische Beziehungen bestehen. Mit diesen mittlerweile 69 - Staaten des a frikanischen, pazifischen und karibischen Raumes, den sogenannten AKP-Staaten (s. Anhang 1), wurden völkerrechtlich verbindliche Verträge vereinbart, die bei einer Laufzeit von zunächst fünf, seit dem jüngsten Abkommen 10 Jahren, sämtliche Bereiche der wirtschaftlichen und entwicklungspolitischen Zusammenarbeit umfassen. Diese AKP-EWG-Abkommen von Lomé - das vierte, Lomé IV, wurde im Dezember 1989 in der Hauptstadt Togos unterzeichnet - sind im Laufe der Zeit auf breiter Basis ausgebaut und erweitert worden.

4 Vgl. O. V.: Ressourcen-Optimierung, in: Handelsblatt, 15. Juni 1992; O.V.: Verstärkte Kooperation für den Technologietransfer, ebenda. 
In den Lome-Abkommen spielen die agrarischen Rohstoffe eine wichtige Rolle, die mineralischen Rohstoffe nehmen dagegen nur eine sekundäre Position ein. Diese Tatsache erklärt sich aus der wirtschaftlichen Dominanz des Agrarsektors in den meisten der 69 AKP-Staaten, wohingegen der Bergbau bei der überwiegenden Zahl der AKP-Staaten nur einen vernachlässigbaren Faktor darstellt.

Bei einer qualitativen Analyse der Lomé-Abkommen verdienen die Stabex- und die Sysmin-Regelung besondere Aufmerksamkeit. "Stabex" steht für "Stabilisierung der Exporterlöse" und "Sysmin" für "Système Minière" (vgl. Abkürzungsverzeichnis).

Beide Verfahren berücksichtigen die Tatsache, daß die Volkswirtschaften einer Großzahl von Entwicklungsländern nach wie vor in erheblichem Umfange von den Exportmöglichkeiten eines oder weniger Rohstoffe abhängen. Plötzliche Preis- oder Absatzschwankungen verursachen daher oft Auswirkungen, die der ohnehin nur mühsamen wirtschaftlichen Entwicklung der betroffenen Länder entgegenwirken. Mit Hilfe der Stabilisierungsverfahren Stabex und Sysmin soll dieser Problematik entgegengewirkt werden.

Stabex, das System zur Stabilisierung von Exporterlösen findet Anwendung, wenn Erlösausfälle bei der Ausfuhr von agrarischen Rohstoffen der AKP-Staaten eintreten und die vereinbarten Abhängigkeits- und Auslöseschwellen erreicht oder überschritten worden sind| Eisenerze und Schwefelkiesabbrände waren die einzigen mineralischen Rohstoffe, die durch die Stabex-Regelung des Lomé-I-Abkommens erfaßt wurden.

In Lomé II ist diesen und 7 weiteren mineralischen Produkten - Kupfer und Kobalt, Phosphate, Mangan, Bauxit und Aluminiumoxid sowie Zinn in der Sysmin-Regelung, dem System für Bergbauerzeugnisse, eine Sonderstellung eingeräumt worden. Seit Lomé III kann dieses Verfahren unter gewissen Bedingungen auch für andere mineralische Rohstoffe angewendet werden.] Weitere Optimierungen sind in der Sysmin-Regelung von Lomé IV vorgenommen worden; unter anderem werden die Mittel aus der Sysmin-Fazilität den AKP-Staaten jetzt als Zuschüsse zur Verfügung gestellt.

Obwohl die Ausfuhr von Bergbauprodukten für die meisten AKP-Staaten nur eine untergeordnete Position einnimmt, war während der Laufzeit von Lomé I deutlich geworden, daß die Bergbauindustrie in einigen wenigen 
AKP-Staaten eine dominierende Rolle einnimmt. Daher ist mit Einführung der Sysmin-Regelung der Versuch unternommen worden, dem Wunsch der AKP-Bergbaustaaten nach einer Stabilisierung der Exporterlöse für ihre mineralischen Rohstoffe entgegenzukommen. Seither wird diesem Wirtschaftszweig in den Lomé-Abkommen eine stärkere Aufmerksamkeit zuteil.

Finanzielle Engpässe und die gegenüber Agrarprodukten anders gearteten Produktions- und Vermarktungsstrukturen verlangten allerdings eine Neukonzeption außerhalb der Stabex-Regelung.

Darüber hinaus sollte mit dem neugeschaffenen Bergbauverfahren auch dem Interesse der Europäischen Gemeinschaft an einer ausgewogenen Rohstoffpolitik Rechnung getragen werden. Diese Zielsetzung ist in den einzelnen Sysmin-Regelungen jedoch immer weiter in den Hintergrund getreten.

Die vorliegende Arbeit befaßt sich mit der Rolle der Sysmin-Fazilität in den AKP-EWG-Abkommen von Lomé.

Die Idee der Arbeit resultiert aus dem Interesse, die noch wenig bekannte Position der Bergbau-Fazilität in den Lome-Abkommen zu definieren. Dies ist aufgrund des Nebencharakters der Sysmin-Regelung und der im Vergleich zum Stabex-Verfahren geringen $\mathrm{ZahJ}$ von Anwendungen bisher nur sehr oberflächlich geschehen (s. Kap. 1.3). Diskussionsbeitrăge, die wegen der unterschiedlichen Interessenslagen häufig subjektiv und emotional geführt wurden, trugen vielfach nicht zu einer wertneutralen Analyse bei.

Insofern erschien es erforderlich, in einer umfassenden Studie die Veränderungen in den Sysmin-Verfahren der einzelnen Lome-Abkommen aufzuzeigen, die bisher nur wenig untersuchten Auswirkungen und Erfahrungen zu erfassen und neue akzeptable Optimierungsmöglichkeiten herauszuarbeiten. 
Die Ziele der Untersuchung sind:

- Die Situation der Märkte für mineralische Rohstoffe in den letzten zwei Jahrzehnten darzustellen, unter besonderer Berücksichtigung der Lage in der Europäischen Gemeinschaft und in den AKP-Staaten.

- Die unterschiedlichen Positionen der einzelnen Lomé-Vertragspartner, das heißt der EG-Kommission, AKP-Staaten und EG-Mitgliedstaaten herauszuarbeiten.

- Die verschiedenen Instrumente und Maßnahmen zugunsten der mineralischen Rohstoffe in den Lomé-Abkommen aufzuzeigen.

- Die bisherigen Aktionen des Sysmin-Verfahrens und ihre Auswirkungen zu analysieren.

- Akzeptable Alternativen zu der gegenwärtigen Sysmin-Regelung darzulegen.

- Den Bezug der Sysmin-Regelung für entwicklungsstrategische Modelle aufzuzeigen.

\subsection{Darlegung der Vorgehensweise}

In der vorliegenden Arbeit wird zunächst in Form einer zusammenfassenden Abhandlung auf die wesentlichen Theorien zur Erklärung von Unterentwicklung sowie die Strategien zur Überwindung von Unterentwicklung eingegangen. Daran anschließend wird die Entwicklungspolitik der Europäischen Gemeinschaft, die Entstehung und der Inhalt der Lomé-Abkommen sowie deren Fortentwicklung bis zum jüngsten Abkommen (Lomé IV) ausführlich geschildert. Es folgt eine detaillierte Darstellung der Märkte für die von der Sysmin-Regelung erfaßten Produkte in den siebziger und achtziger Jahren, wobei besonders auf die exportwirtschaftlichen Aspekte für die AKP-Bergbauländer und auf die versorgungspolitischen Aspekte für die Europäische Gemeinschaft eingegangen wird.

Bereits durch die intensive Auseinandersetzung mit den recht komplexen Durchführbarkeitsbestimmungen können die Schwächen der Sysmin-Regelung aufgezeigt werden. Die kritische Gegenüberstellung von Erwar- 
tungen und Ansprüchen mit der bisherigen Praxis - dies wurde durch empirische Erhebungen in den Abbaubetrieben der Zambia Consolidated Copper Mines, wo es zur ersten Anwendung der Sysmin-Regelung kam, möglich - macht deutlich, wo es zu einer Diskrepanz zwischen Theorie und Realität kommt.

Das Herausarbeiten von Möglichkeiten zur Verbesserung des Sysmin-Verfahrens in Form von Erweiterungen, Ergänzungen oder anderen Modifikationen läßt erneut die Schwächen aber auch die Stärken der Sysmin-Regelung deutlich werden.

Eine Vielzahl von Gesprächen mit Repräsentanten der verschiedenen Lomé-Vertragspartner und ein umfangreiches Literaturstudium bilden die Grundlage dieser Untersuchung. Auf diese Weise ist versucht worden, der vorliegenden Arbeit einen möglichst objektiven Charakter zu geben.

Es erscheint wichtig an dieser Stelle festzuhalten, daß die einzelnen, zum Teil gegensätzlichen Standpunkte der Lomé-Unterzeichner singulär gesehen durchaus ihre Berechtigung haben, im Kontext betrachtet spiegeln sie jedoch nur einseitige Blickwinkel wider.

Neben Unterredungen bei der EG-Kommission in Brüssel, deutschen Bundesministerien und anderen Institutionen wurden während zwei Aufenthalten in Sambia empirische Daten bei der staatlichen Kupfergesellschaft Zambia Consolidated Copper Mines (ZCCM) erhoben, wozu 5 der damals noch 7 Produktionsabteilungen besucht wurden. Die ZCCM bekam als erstes Unternehmen Darlehen aus der Bergbaufazilität Sysmin des Lome-II-Abkommens zugesprochen. Im Jahre 1985 wurden weitere Mittel genehmigt, zur Zeit wird ein dritter Antrag von den zuständigen Gremien bearbeitet. Daher war das Unternehmen für die Ermittlung der Akzeptanz und Effizienz der Sysmin-Regelung prädestiniert.

Durch die Beschränkung der empirischen Untersuchung auf nur eine Bergbaugesellschaft, einen AKP-Staat und die mineralischen Rohstoffe Kupfer und Kobalt erscheint die allgemeine Aussagekraft der Arbeit eingeschränkt zu sein. Aufgrund der diversen Gespräche und Literaturstudien ist es jedoch gelungen, die in Sambia gesammelten Daten zu extrapolieren und so die entscheidenden Engpässe der Regelung für Bergbauerzeugnisse herauszuarbeiten. In diesem Zusammenhang ist zu erwähnen, daß die in dieser Arbeit getroffenen Optimierungsvorschläge auch für die neueste Sysmin-Regelung des Lomé-IV-Abkommens Gültigkeit haben. 
In der Arbeit wird die Bezeichnung "Europäische Gemeinschaft" (EG) synonym mit Europäischen Gemeinschaften - verwendet, obwohl die Abkommen von Lomé offiziell als AKP-EWG-Abkommen bezeichnet werden, also mit der Europäischen Wirtschaftsgemeinschaft abgeschlossen wurden.

Durch den Vertrag vom 8. April 1965 sind mit Wirkung vom 1. Juli 1967 die Ministerräte und die Exekutivorgane der 3 Teilgemeinschaften: die Hohe Behörde der Europäischen Gemeinschaft für Kohle und Stahl, die Kommission der Europäischen Wirtschaftsgemeinschaft und die Kommission der Europäischen Atomgemeinschaft zu einem gemeinsamen Rat, zur Kommission der Europäischen Gemeinschaften zusammengefaßt worden. Der Erfolg und die Akzeptanz der Verschmelzung der Organe dokumentiert sich auch in der zunehmenden Verwendung des Begriffes Europäische Gemeinschaft. In der vorliegenden Arbeit wird daher nur dann auf die Europäische Wirtschaftsgemeinschaft oder eine der anderen Teilgemeinschaften eingegangen, wenn dies explizit erforderlich ist.

\subsection{Bisherige Forschungsansätze}

Bedingt durch die im Gesamtkomplex der Lomé-Abkommen zweitrangige Bedeutung der Sysmin-Regelung und die im Vergleich zu dem StabexVerfahren geringere Zahl von Anwendungsfällen findet man zu dem Verfahren für Bergbauerzeugnisse nur gelegentlich Kommentare, beziehungsweise Untersuchungen. Bei der Vielzahl von Abhandlungen über die Lomé-Abkommen - meistens wird die Erlösstabilisierung der Stabex-Regelung analysiert - ist es trotzdem verwunderlich, daß die mineralischen Rohstoffe derart unterrepräsentiert sind.

Zwar wird in solchen Studien die Existenz einer Regelung für Bergbauerzeugnisse erwähnt, selten enthalten sie jedoch eine ausführliche Schilderung über das Prozedere der Sysmin-Fazilität. Bei den kurz nach der Einführung des Systems erschienenen Artikeln fällt auf, daß oft nur oberflächlich recherchiert worden war und daher das nicht ganz unkomplizierte Verfahren unrichtig oder unvollständig wiedergegeben wurde.

Dagegen werden in Untersuchungen, die nach der Unterzeichnung von Lomé III veröffentlicht wurden, häufig nur die Veränderungen gegenüber der Sysmin-Regelung des Lomé-II-Abkommens aufgeführt. Abhandlungen 
in dem AKP-EWG-Magazin "The Courier" haben sich gelegentlich mit dem Sysmin-Verfahren auseinandergesetzt, ${ }^{5}$ allerdings wurden in diesen Fällen nur einzelne Fragestellungen aufgegriffen. Selbst in einem umfassenden Bericht der EG-Kommission über die Durchführung der finanziellen und technischen Zusammenarbeit im Rahmen der ersten beiden LomeAbkommen ist das Sysmin-Verfahren nur zwei Mal aufgeführt. Dabei wird die Regelung kurz beschrieben, und es werden die bisher genehmigten Transfer-Zahlungen aufgelistet. ${ }^{6}$ Erst im Jahre 1986 legte die EGKommission in einer kurzen, internen Abhandlung an den Europarat das komplizierte Sysmin-Beschlußfassungsverfahren näher dar. ${ }^{7}$ Auch in Publikationen zu dem Lomé-IV-Abkommen werden die Modifikationen der Sysmin-Regelung gar nicht oder nur sehr oberflächlich diskutiert. ${ }^{8}$ Gelegentlich sind auch noch in den neueren Veröffentlichungen unkor-

5 Vgl. u.a.: O.V.: First Sysmin operation, in: The Courier, Nr. 73, Mai - Juni 1982, S. gelb I; Grand, B.: Sysmin, in: The Courier, Nr. 79, Mai - Juni 1983, S. 7 - 8; O.V.: Negotiations update, in: The Courier, Nr. 85, Mai - Juni 1984, S. gelb II f.; Pagni, L.: Getting out of the copper straitjacket, in: The Courier, Nr. 85, Mai - Juni 1984, S. 18 f.; Grand, B.: Sysmin-Mines as instruments of international cooperation, in: The Courier, Nr. 94, November - Dezember 1985, S. 82 - 85; Kommission der EG/Europäische Investitionsbank: Financing of mining projects, in: The Courier, Nr. 109, Mai - Juni 1988, S. 11 - 14.

6 Vgl. Kommission der EG (Hrsg.): Zehn Jahre Lome, Geschichte der Partnerschaft AKP-EWG 1976 - 1985, SEK (86) 1295, Brüssel, 22. Juli 1986, S. 22 und 39.

7 Vgl. Kommission der EG (Hrsg.): Beschlußfassungsverfahren für die besondere Finanzierungsfazilität für Bergbauerzeugnisse gem. Titel II, Kapitel III des dritten AKP-EWG-Abkommens, Kom (16) 183 endg., Brüssel, 10. April 1986.

8 Vgl. Stevens, C.: Die Lehren aus Lomé III, in: epd-Entwicklungspolitik: Vorausinformation 7/88, Frankfurt/M. 1988; Schmuck, O.: Lomé-Zusammenarbeit auf neuer Grundlage, in: Entwicklung + Zusammenarbeit 3/90, S. 16 - 17; O.V.: Lomé-IV-Abkommen weitgehend in Kraft, in: Commerzbank, Außenhandelsblätter 4/90, S. 7 - 8; Oyowe, A.: A convention for a changing world, in: The Courier, Nr. 120, März - April 1990, S. 2 - 4. 
rekte beziehungsweise unpräzise Aussagen über die Sysmin-Regelung anzutreffen 9 .

Die europäische Rohstoffversorgungspolitik ist zuletzt im Jahre 1972 in einem umfassenden Memorandum dargestellt und analysiert worden. ${ }^{10}$ Eine Neuauflage dieser ausfuihrlichen Studie ist vorläufig - aufgrund der seit Beginn der achtziger Jahre entspannten Lage bei der Rohstoffversorgung - nicht vorgesehen.

In einem Bericht an den EG-Ministerrat geht die EG-Kommission im Jahre 1983 auf die Lage der EG-Rohstoffversorgung und den AKP-Bergbau ein. ${ }^{11}$ Erstaunlich ist, daß in den dort publizierten Tabellen kein aktuelles Zahlenmaterial Verwendung findet. Auch wird nicht auf die Bergbau-Regelung des Lomé-II-Abkommens und etwaige Alternativen eingegangen.

Eine in diesem Sinne weiterführende Studie wurde vom Overseas Development Institute, London publiziert, ${ }^{12}$ wobei aber auch in diesem Falle dem Stabex-Verfahren Priorität eingeräumt wird. In einem UNCTAD-Bericht wird unter anderem das langwierige Prozedere der Sysmin-Regelung kritisiert, ${ }^{13}$ auf die daraus resultierenden Schwierigkeiten und die Möglichkeiten zu deren Vermeidung wird jedoch nicht näher eingegangen.

9 Beispielsweise wird behauptet, Sysmin sein ein Verfahren zur Exporterlösstabilisierung, vgl. Lingnau, H.: Neue Elemente in der Entwicklungszusammenarbeit der Europäischen Gemeinschaft zu Beginn der 90er Jahre, DIE, Berlin, Mai 1991, S. 19, oder es wird die nicht mehr aktuelle Version von Lomé II zitiert, vgl. Nuscheler, F.: Lernund Arbeitsbuch Entwicklungspolitik, 3. Aufl., Bonn 1991, S.307.

10 Vgl. Michaelis, M .: Memorandum über eine Europäische Rohstoffversorgungspolitik, Gutachten im Auftrag der Kommission der EG, o.O., September 1972.

11 Vgl. Kommission der EG (Hrsg.): Communication de la Commission au Conseil: Les relations de la Communaute Européene et les Etats ACP dans le domaine minier, Com (83) 651 final, Brüssel, 8. November 1983.

$12 \mathrm{Vgl}$. Stevens, C.: EEC and Third World, A Survey 4: Renegotiating Lomé, London 1984.

13 Vgl. UNCTAD (Hrsg.): Les systemes Stabex et Sysmin, TD/B/C.1/237, Genf, 26. Januar 1983, S. $42-48$. 
Auch in einer im Jahre 1984 vom Bundesministerium fuir wirtschaftliche Zusammenarbeit herausgegebenen Studie über Exportstabilisierungsmaßnahmen wird die kurze Abhandlung der Sysmin-Fazilität mit "bisher nur sehr geringen Erfahrungen" begründet. ${ }^{14}$ Während der Vorbereitungsphase für die Verhandlungen zu Lome IV wurden gelegentlich kritische Studien verfaßt, ${ }^{15}$ die zum Teil jedoch nur als unveröffentlichte Arbeitspapiere dienten. Derartige Analysen befassen sich in der Regel nur mit einzelnen Aspekten oder werden aus einem bestimmten Blickwinkel heraus erstellt (z.B. Gruppe der Europäischen Metallindustrie).

Während auf der einen Seite die unzureichende Kenntnis über das SysminVerfahren bedauert wurde, hatte auf der anderen Seite bis zum Sommer 1984 - mehr als zwei Jahre nach der Zuteilung - noch kein Kommentator die Möglichkeit genutzt, vor Ort - in den begünstigten Bergbaubetrieben Sambias - Informationen zu sammeln. Wie bei sämtlichen Gesprächen in den einzelnen Abbaubetrieben der Zambia Consolidated Copper Mines (ZCCM) von den sambischen Gesprächspartnern betont wurde, hatte sich bis zu diesem Zeitpunkt kein Besucher nach den Auswirkungen, Engpässen oder Verfahrensschwierigkeiten der Sysmin-Regelung erkundigt. Dies traf sowohl für Gäste zu, die in ihrer Funktion als Regierungsvertreter eines EG-Mitgliedsstaates kamen, als auch für Repräsentanten von Industrie- und Handelsunternehmungen beziehungsweise von wissenschaftlichen Institutionen. Erst nach Beantragung eines dritten SysminTransfers durch Sambia wurde von der EG-Kommission eine Studie in Auftrag gegeben, welche die Verwendung der bisher eingesetzten SysminMittel untersuchen sollte.

Die anfangs getroffene Behauptung, daß die Sysmin-Regelung in den Augen vieler unberechtigterweise nur eine sekundäre Rolle in dem vielfältigen Entwicklungsinstrumentarium der Lomé-Abkommen spielt, wird durch die geringe Aufmerksamkeit, die dem Thema in der Lomé-Literatur widerfährt, bestätigt.

14 Vgl. Borrmann, A., et al.: Ausgewählte Modelle und Reformmöglichkeiten der Exporterlösstabilisierung, Forschungsberichte des BMZ, 56, Köln 1984.

15 Vgl. u.a.: Maennig, W.: Zur Kritik des EG-Sonderfonds für Bergbauerzeugnisse (Sysmin), in: Metall, 41. Jg., Heft 10, Oktober 1987, S. 1042 - 1046; Eurometaux (Hrsg.): Lome IV-Sysmin, Comments of the non-ferrous metals industry; unveröffentlichter Entwurf, Brüssel, 21. September 1988. 


\section{DIE AKP-EWG-ABKOMMEN VON LOMÉ}

\subsection{Darstellung von Theorien zur Erklärung von Unterentwicklung sowie von Strategien zur Überwindung von Unterentwicklung}

Seit den sechziger Jahren sind die unterschiedlichsten Interpretationen beziehungsweise Theorien über die Situation in den Entwicklungsländern aufgestellt worden (Entwicklungstheorien). Darüber hinaus wurden Konzepte und Strategien entwickelt, die aufzeigen sollten, wie die Unterentwicklung in der Dritten Welt zu überwinden sei.

Durch historische Entwicklungen wurden einige Entwicklungstheorien beziehungsweise ihre wesentlichen Ansätze als nicht relevant widerlegt, weil die Argumentationsführung entweder politisch einseitig orientiert war oder empirischen Untersuchungen nicht standhielt.

Generell läßt sich die Aussage treffen, daß es eine allgemeine Entwicklungstheorie zur Lage in den Entwicklungsländern nicht gibt. ${ }^{1}$ Vielmehr sind Konstellationen denkbar, wo sogar in einem Entwicklungsland mehrere entwicklungstheoretische Interpretationen zum Ansatz kommen können.

Diese Tatsache erklärt auch, weshalb eine Vielzahl von Lösungsansätzen und Strategien zur Überwindung der Unterentwicklung existieren. Eine allgemeine, für sämtliche Entwicklungsländer gültige Strategie gibt es wie auch bei den Theorien zur Erklärung der Unterentwicklung - nicht.

Im nachfolgenden werden die für diese Arbeit wesentlichen Entwicklungstheorien vorgestellt sowie Lösungsansätze zur Behebung der Unterentwicklung aufgezeigt.

1 Vgl. Hemmer, H.R.: Wirtschaftsprobleme der Entwicklungsländer, 2. Auflage, München 1988, S. $119 \mathrm{f}$. 


\subsubsection{Theorien zur Erklärung von Unterentwicklung (Entwicklungs- theorien)}

Die Entwicklungstheorien lassen sich, je nachdem welche Ursache für die unbefriedigende Situation in den Entwicklungsländern zugrundegelegt wird, in unterschiedliche Gruppen zusammenfassen:2

1) interne Ursachen: a) Faktorbestandsdefizite und -engpässe,

b) Strukturprobleme,

c) schnelles Bevölkerungswachstum.

2) externe Ursachen:

a) historische Dependenzen,

b) internationale Wirtschaftsbeziehungen.

ad 1)

a) Eine unzureichende Faktorausstattung kann zu erheblichen Hemmnissen bei der wirtschaftlichen Entwicklung eines Landes führen. Im nachfolgenden wird nicht näher auf die Problematik einer unzureichenden Rohstoffausstattung beziehungsweise widriger klimatischer Bedingungen eingegangen, da sich diese Arbeit mit Entwicklungsinstrumenten auseinandersetzt, die für AKP-Staaten mit ausreichender Rohstoffausstattung konzipiert wurden.

Es ist unumstritten, daß Defizite im Faktor Kapital, das heißt in der Kapitalausstattung wie in der Kapitalbildung, eine angemessene wirtschaftliche Entwicklung verhindern. ${ }^{3}$ Statistische Vergleiche dokumentieren, daß in Industrieländern eine durchschnittlich zwei- bis dreimal größere Kapitalbildung erfolgt als in Entwicklungsländern. ${ }^{4}$

2 Vgl. Hemmer, H.R.: Wirtschaftsprobleme der Entwicklungsländer, a.a.O., S. 120.

3 Vgl. Kaiser, M.; Wagner, N.: Entwicklungspolitik: Grundlagen - Probleme - Aufgaben, Heidelberg 1986, S. 83 f; Ochel, W.: Die Entwicklungsländer in der Weltwirtschaft, Köln 1982, S. 69 ff.

4 Vgl. Hemmer, H.R.: Wirtschaftsprobleme der Entwicklungsländer, a.a.O., S. $140 \mathrm{f}$. 
Es ist darauf hinzuweisen, daß sich die Definition des Begriffes Kapital nicht allein auf "Sach"-Kapital beschränkt, sondern auch das "Human"Kapital miterfaßt. ${ }^{5}$

Viele Entwicklungsländer sehen sich nicht in der Lage, das Defizit an Humankapital durch den vermehrten Einsatz von anderen Produktionsfaktoren auszugleichen, da einerseits der Substituierbarkeit des Humankapitals Grenzen gesetzt sind, andererseits die in den Produktionsprozessen eingesetzte Arbeit zunehmend anspruchsvoller wird. Unterschiede in der Ausstattung mit Humankapital trugen, so wurde in empirischen Untersuchungen festgestellt, in stärkerem Maße zu Differenzen im Pro-Kopf-Einkommen bei als unterschiedliche Ausstattungen mit Sachkapital oder natürlichen Ressourcen. ${ }^{6}$

Die unzureichende Ausstattung mit dem Produktionsfaktor Kapital läßt sich im wesentlichen als Folge einer ungenügenden Ersparnisbildung beziehungsweise ungenügender produktiver Investitionen erklären. Als Ursachen werden mangelnde Fähigkeit oder mangelnde Bereitschaft aufgeführt, Ersparnisse zu bilden beziehungsweise Investitionen zu tätigen. Diese Verhaltensweisen sind beispielsweise anzutreffen, wenn pessimistische Gewinnerwartungen oder institutionelle Hemmnisse bestehen.?

b) Nach verbreiteter Auffassung bedingen auch Strukturprobleme aufgrund der Polarisierung von Gesellschaften in traditionelle und moderne Bereiche eine unzureichende wirtschaftliche Entwicklung. ${ }^{8}$ Durch die Spaltung zwischen dem modernen Bereich mit häutig komplexen Arbeitsprozessen sowie kapitalintensiven Techniken und dem traditionellen Bereich mit in der Regel einfachen Produktionstech-

5 Vgl. Hemmer, H.R.: Wirtschaftsprobleme der Entwicklungsländer, a.a.O., S. 142 f.; Ochel, W.: Die Entwicklungsländer in der Weltwirtschaft, a.a.O., S. 70.

- Vgl. Hemmer, H.R.: Wirtschaftsprobleme der Entwicklungsländer, a.a.O., S. 143.

7 Vgl. Nuscheler, F.: Lern- und Arbeitsbuch Entwicklungspolitik, a.a.O., S. $82 \mathrm{f}$.

$8 \mathrm{Vgl}$. ebenda, S. 81 f; Hemmer, H. R.: Wirtschaftsprobleme der Entwicklungsländer, a.a.O., S. 189; Ochel, W.: Die Entwicklungsländer in der Weltwirtschaft, a.a.O., S. 76 f.; Grimm, K.: Theorien der Unterentwicklung und Entwicklungsstrategien, Opladen 1979, S. $46 \mathrm{ff}$. 
niken sowie geringer Kapitalausstattung werden Transmissionen von modernen zum herkömmlichen Bereich erschwert. Durch zunehmend unterschiedliche Einkommensverhältnisse, Ausbildungsniveaus, soziale Verhaltensweisen, aber auch unterschiedliche ökonomische Randbedingungen (z.B. Kapitalbeschaffungsmöglichkeiten), entsteht ein wirtschaftlicher Dualismus, der eine ausgewogene Entwicklung eines Landes unmöglich macht.

c) Das Bevölkerungswachstum in den Entwicklungsländern, es liegt in der Regel deutlich über dem der Industrieländer, wird nach herrschender Meinung in den überwiegenden Fällen ebenfalls als entwicklungshemmende Größe eingestuft.9 Obwohl ein Anstieg des Arbeitskräftepotentiales zu verzeichnen ist, fehlen in den meisten Ländern diejenigen Faktoren, die komplementär zur Arbeit benötigt werden, um entwicklungsfördernde Effekte zu erzielen. Die Folge ist ein sinkendes Pro-Kopf-Einkommen. Diese Tatsache wird noch dadurch verstärkt, daß insbesondere der Anteil der Jugendlichen an der Bevölkerung zunimmt, wodurch zunächst umfangreiche - private wie staatliche - Investitionen zur Ernährung, Ausbildung etc. erbracht werden müssen. ${ }^{10}$ Entwicklungshemmende Effekte aufgrund einer beispielsweise unzureichenden Kapitalausstattung (s.a. 1.a) werden so verstärkt.

a) Mehrere Wirtschaftstheoretiker versuchten die wirtschaftshistorische Entwicklung von Staaten in idealisierten Wirtschafts- beziehungsweise Gesellschaftsstadien darzustellen. Als die wesentlichen Stufenlehren der Entwicklung sind die beiden Theorien von Marx und Rostow zu bezeichnen. ${ }^{11}$ Die zunächst für die Industriestaaten aufgestellten Theorien wurden später an die Situation der Entwicklungsländer adaptiert. Dabei ist von der Prämisse ausgegangen worden, daß jedes Land in je-

9 Vgl. Kaiser, M.; Wagner, N.: Entwicklungspolitik, a.a.O., S. 86 f.

${ }^{10} \mathrm{Vgl}$. Hemmer, H.R.: Wirtschaftsprobleme der Entwicklungsländer, a.a.O., S. 273 und $282 \mathrm{f}$.

11 Rostow, W.W.: Stadien des wirtschaftlichen Wachstums. Eine Alternative zur marxistischen Entwicklungstheorie, Göttingen 1960; vgl.: Kaiser, M.; Wagner, N.: Entwicklungspolitik, a.a.O., S. 124. 
weils individuellem Tempo die jeweiligen Wirtschaftsstufen zu absolvieren habe. ${ }^{12}$

Während in der marxistischen Theorie die historischen Beziehungen zwischen den entwickelten und den weniger entwickelten Staaten beziehungsweise Gesellschaftsformen (Kolonialismus) über die Ausbeutungsthese erklärt wird, unterstellt Rostow der Kolonialisierung sogar eine Anregung der - durch interne Impulse nicht ausreichend geförderten - Entwicklung in den Kolonien. ${ }^{13}$ Beide Thesen, wobei besonders die erstere in Form von Abhängigkeitstheorien weiterentwickelt wurde, sind so nicht haltbar beziehungsweise lassen sich nicht für die gesamte Gruppe der Entwicklungsländer verallgemeinern. ${ }^{14}$

So gibt es ehemalige Kolonien, die sich trotz einer gewissen Ausbeutung durch ihre einstmalige Kolonialmacht zu einem Industrieland entwickelt haben. Zum anderen lassen sich genügend Beispiele von ehemaligen Kolonien auffuihren, die keine hinreichende Entwicklung erfahren haben. ${ }^{15}$

b) Eine beachtliche Anzahl von Autoren sieht in den bestehenden Wirtschaftsbeziehungen zwischen den Industrie- und Entwicklungsländern (Nord-Süd-Beziehungen) ein ausschlaggebendes Hemmnis für eine akzeptable Entwicklung in der Dritten Welt, da die Entwicklungsanstrengungen in den Entwicklungsländern durch die starken, für sie negativen Auswirkungen dieser Wirtschaftsbeziehungen gedämpft oder sogar zunichte gemacht würden. ${ }^{16}$ Als entwicklungshemmende Kontereffekte werden u.a. Faktorabwanderungen - auch Arbeitskräfte -, eine Verschlechterung der Terms of Trade sowie Exporterlösschwankungen bei

${ }^{12} \mathrm{Vgl}$. Hemmer, H.R.: Wirtschaftsprobleme der Entwicklungsländer, a.a.O., S. 94 f.; Grimm, K.: Theorien der Unterentwicklung und Entwicklungsstrategien, a.a.O., S. $134 \mathrm{ff}$.

${ }^{13} \mathrm{Vgl}$. Hermmer, H.R.: Wirtschaftsprobleme der Entwicklungsländer, a.a.O., S. $109 \mathrm{ff}$.

${ }^{14} \mathrm{Vgl}$. Nuscheler, F.: Lern- und Arbeitsbuch Entwicklungspolitik, a.a.O., S. 89.

15 Vgl.: Kaiser, M; Wagner, N.: Entwicklungspolitik, a.a.O., S. 114.

${ }^{16} \mathrm{Vgl}$. Hemmer, H.R.: Wirtschaftsprobleme der Entwicklungsländer, a.a.O., S. 205; Grimm, K.: Theorien der Unterentwicklung und Entwicklungs-strategien, a.a.O., S. $54 \mathrm{ff}$. 
Primärerzeugnissen aufgeführt, wodurch sich die Diskrepanz zwischen den beiden Ländergruppen weiter verstärken würde. ${ }^{17}$

Auch solche Aussagen haben in dieser Form als allgemeingültige These keine Gültigkeit. Häufig sind die Kontereffekte nicht den internationalen Wirtschaftsbeziehungen zuzuschreiben, sondern müssen in den internen Defiziten des jeweiligen Landes gesucht werden. Prinzipiell läßt sich feststellen, daß der Außenhandel eines Landes sowohl entwicklungsfördernd als auch entwicklungsneutral oder sogar entwicklungshemmend sein kann. ${ }^{18}$

\subsubsection{Strategien zur Überwindung von Unterentwicklung (Entwick- lungsstrategien)}

Ähnlich wie die Entwicklungstheorien lassen sich auch die Entwicklungsstrategien in verschiedenen Gruppen zusammenfassen. Im nachfolgenden wird näher auf die sektoralen und die zielgruppenorientierten Strategien eingegangen. Nicht näher erläutert werden hier die gelegentlich als regionale Entwicklungsstrategien bezeichneten Konzepte, ${ }^{19}$ da sich diese aus den beiden anderen Gruppen ableiten lassen.

Als die traditionellen Konzepte unter den sektoralen Strategien sind die Strategie des ausgewogenen Wachstums (balanced growth) und die Strategie des unausgewogenen Wachstums (unbalanced growth) anzusehen.

Die zuerst genannte Strategie hat eine zueinander ausgewogene Entwicklung sämtlicher Wirtschaftszweige des jeweiligen Landes zum Ziel, um so die Arbeitsproduktivität, das Realeinkommen sowie die Absatzmärkte insgesamt anzuheben beziehungsweise zu vergrößern. Das Ziel der Strate-

${ }^{17}$ Vgl. Hemmer, H.R.: Wirtschaftsprobleme der Entwicklungsländer, a.a.O., S. 220 ff. und S. 238 ff; Kaiser, M; Wagner, N.: Entwicklungspolitik, a.a.O., S. 115.

${ }^{18}$ Vgl. Hemmer, H.R.: Wirtschaftsprobleme der Entwicklungsländer, a.a.O., S. 243.

${ }^{19}$ Ebenda, S. $587 \mathrm{ff}$. 
gie des unausgewogenen Wachstums dagegen ist, durch Ungleichgewichtssituationen in Teilmärkten neue ökonomische Impulse zu geben. ${ }^{20}$

Wie Erfahrungen zeigen, lassen sich beide Strategien in Ansätzen durchaus erfolgreich realisieren, vorausgesetzt es gibt keine Randbedingungen, durch welche der Entwicklungsprozeß behindert wird. Als eine Hauptschwäche beider Konzepte wird das Nichtberücksichtigen der internationalen Wirtschaftsbeziehungen angesehen. ${ }^{21}$

Zwei Strategien, welche die externen Wirtschaftsbeziehungen berücksichtigen, sind die sogenannte Exportförderungs- und die Importsubstitutionsstrategie.

Die Exportförderungsstrategie hat zum Ziele, durch eine Steigerung der Exporterlöse verstärkt Devisen zu erwirtschaften, die zum Import von im Lande nicht produzierten, jedoch benötigten Produkten oder Dienstleistungen eingesetzt werden können. Darüber hinaus sollen direkte EntwickJungseffekte erzielt werden, indem Impulse von der exportorientierten Wirtschaft auf die übrigen wirtschaftlichen Aktivitäten des jeweiligen Landes übertragen werden. ${ }^{22}$

Die Importsubstitutionsstrategie will erreichen, daß durch eine Förderung der heimischen Industrie Produkte erzeugt und Dienstleistungen offeriert werden können, die bislang importiert werden mußten. Neben Impulsen, die auf die anderen Wirtschaftssektoren einwirken, können so die Einfuhren von diversen Produkten reduziert werden. Daraus resultiert ein Rückgang der Devisenausgaben bezjehungsweise die Devisen können für andere, nicht substituierbare Güter eingesetzt werden. ${ }^{23}$

${ }^{20} \mathrm{Vgl}$. Grimm, K.: Theorien der Unterentwicklung und Entwicklungsstrategien, a.a.O., S. 90 ff; Hemmer, H.R.: Wirtschaftsprobleme der Entwicklungsländer, a.a.O., S. 438 f. und S. $447 \mathrm{f}$.

${ }^{21}$ Ebenda, S. $460 \mathrm{f}$.

$22 \mathrm{Vgl}$. Hemmer, H.R.: Wirtschaftsprobleme der Entwicklungsländer, a.a.O., S. 509 f; Grimm, K.: Theorien der Unterentwicklung und Entwicklungsstrategien, a.a.O., S. $106 \mathrm{f}$.

${ }^{23} \mathrm{Vgl}$. Hemmer, H.R.: Wirtschaftsprobleme der Entwicklungsländer, a.a.O., S. 527; Grimm, K.: Theorien der Unterentwicklung und Entwicklungsstrategien, a.a.O., S. $102 \mathrm{f}$. 
Beide Strategien gehen davon aus, daß die im jeweiligen Entwicklungsland erzeugten Produkte und Dienstleistungen wettbewerbsfähig sind, entweder im Ausland (Exportförderung) oder im Inland (Substitution). ${ }^{24}$

Die zielgruppenorientierten Strategien zielen primär nicht auf das Wachstum ökonomischer Strukturen, sondern sollen eine Verbesserung der Verhältnisse, besonders der ärmeren Bevölkerung in Entwicklungsländern bewirken.

Die Strategie der Grundbedürfnisbefriedigung zählt zu den traditionellen, zielgruppenorientierten Strategien, im Laufe der Zeit kamen weitere Konzepte wie die Anhebung der Produktivität der Armen oder Erhöhung der Beschäftigung hinzu. Seit den achtziger Jahren werden diese Strategien unter dem Begriff "Entwicklung menschlicher Ressourcen" zusammengefaßt. ${ }^{25}$

Pauschale Aussagen über die Qualtitäten der einzelnen Strategien zur Überwindung der Unterentwicklung sollen hier nicht getroffen werden. Genauso wenig kann an dieser Stelle über die Zweckmäßigkeit des Einsatzes der einen oder anderen Strategie beziehungsweise über die gemeinsame Umsetzung mehrerer Strategien diskutiert werden. Wie die obige Darstellung deutlich werden läßt, gibt es die unterschiedlichsten Alternativen, wo Maßnahmen gegen die Unterentwicklung ansetzen können.

Jedoch heißt es nicht zwangsläufig, daß eine bereits erfolgreich umgesetzte Strategie in einem anderen Falle ebenfalls positive Effekte erzeugt. Vielmehr sind, unter Berücksichtigung der jeweiligen Rahmenbedingungen, individuelle Konzepte zu erstellen, worin die einzelnen Strategien auf die kurz- und langfristigen Entwicklungsziele des einzelnen Landes abzustimmen und zu einer Gesamtstrategie zusammenzufügen sind.

$24 \mathrm{Vgl}$. Hemmer, H.R.: Wirtschaftsprobleme der Entwicklungsländer, a.a.O., S. 532 f.

25 Vgl. Hemmer, H.R.: Wirtschaftsprobleme der Entwicklungsländer, a.a.O., S. 553 ff; Kaiser, M.; Wagner, N.: Entwicklungspolitik, S. $129 \mathrm{ff}$. 


\subsection{Die Grundsätze der Entwicklungspolitik der Europäischen Ge- meinschaft}

Mit Unterzeichnung der Römischen Verträge zur Gründung der Europäischen Wirtschaftsgemeinschaft (EWG) im Jahre 1957 wurde auf Betreiben Frankreichs auch ein Partnerschaftsverhältnis mit einem Teil der damals noch nicht unabhängigen Länder in Übersee begründet. In einem Zusatzprotokoll zu den Römischen Verträgen wurden auch Marokko und Tunesien aufgrund ihrer engen Beziehungen zu Frankreich besonders erwähnt.

Die zum Teil noch von den 6 EWG-Gründerstaaten abhängigen Länder und Gebiete wurden in eine Art Freihandelszone mit der EWG assoziiert. Im Jahre 1958 errichtete die Gemeinschaft den ersten Europäischen Entwicklungsfonds (EEF), der diesen zum Großteil in Afrika befindlichen, sogenannten Überseeischen Ländern und Gebieten (ÜLG) zugute kam. ${ }^{26}$

Der Begriff "Entwicklungszusammenarbeit" erscheint in den Römischen Verträgen noch nicht. Bis in die siebziger Jahre hinein war daher die Kompetenz der EG-Kommission auf die Handelspolitik beschränkt, die Konzeption einer finanziellen und technischen Zusammenarbeit (Auswärtige Beziehungen) behielten sich die einzelnen EG-Mitgliedstaaten vor. Erst mit der sogenannten Erklärung von Paris im Oktober 1972 wurde der Weg für eine globale Entwicklungspolitik der EG-Kommission geöffnet.

Die EG-Kommission erhielt das Mandat sowohl zur Fortführung der $\mathrm{Zu}$ sammenarbeit im regionalen Rahmen als auch zum Aufbau einer weltweiten Entwicklungspolitik, besonders für die ärmeren Länder in Lateinamerika und Asien. ${ }^{27}$

Mit dem im Jahre 1973 vollzogenen Beitritt des Vereinigten Königreiches, Dänemarks und Irlands zur Europäischen Gemeinschaft, der Unabhängigkeit früherer Kolonien, dem wachsenden Selbstbewußtsein der Entwick-

${ }^{26}$ Vgl. Kommission der EG (Hrsg.): Europa - Dritte Welt, ein Dialog, Luxemburg 1989, S. 22 f.; Ochel, W.: Die Entwicklungsländer in der Weltwirtschaft, a.a.O., S. 219.

${ }^{27}$ Vgl. Kommission der EG (Hrsg.): Europa - Dritte Welt, a.a.O., S. 70 und $76 \mathrm{f}$.; Derselbe: Die europäische Gemeinschaft in der Welt, Luxemburg 1988, S. 2 f. 
lungsländer sowie der Erkenntnis der gegenseitigen Abhängigkeit wurde die Entwicklungszusammenarbeit allmählich auf ganz Afrika, die Karibik, den Pazifik, den Mittelmeerraum, Asien und Lateinamerika ausgedehnt. ${ }^{28}$

Das Kernstück der europäischen Entwicklungszusammenarbeit ist in den Abkommen von Lomé zu sehen. Dies erklärt sich aus dem besonderen Verhältnis der Europäischen Gemeinschaft zu den Ländern des afrikanischen, karibischen und pazifischen Raumes (AKP-Staaten). Ein weiterer Schwerpunkt der Europäischen Entwicklungszusammenarbeit liegt in den Assoziierungs- und Kooperationsverträgen mit den Mittelmeeranrainern. Sofort nach der Gründung der EWG im Jahre 1958 waren sämtliche unabhängigen Länder des Mittelmeerraumes um einen freien Zugang zum EWG-Markt bemübt. So entstanden ab dem Jahre 1963 nach und nach bilaterale Abkommen mit den Mittelmeeranrainern, die eine Annäherung an die Europäische Gemeinschaft wünschten.

Erst ab Beginn der siebziger Jahre wurden dann, basierend auf dem EGKonzept der "globalen Mittelmeer-Politik", bilaterale Abkommen geschlossen, die bis auf Jugoslawien (Sonderabkommen) für die nördlichen Mittelmeeranrainer ein Assoziierungsverhältnis zur Europäischen Gemeinschaft begründeten. Mit den südlichen und östlichen Ländern des Mittelmeerraumes konnten in der zweiten Hälfte der siebziger Jahre globale Kooperationsabkommen geschlossen werden. Die Abkommen hatten im wesentlichen Handelspräferenzen sowie technische und finanzielle Hilfe zum Inhalt und waren zeitlich unbefristet. Kollektive, überregionale Ansätze wie für die AKP-Staaten ließen die historischen und politischen $\mathrm{Be}$ dingungen in der Region nicht $\mathrm{zu}^{29}$

Durch die Süderweiterung der Gemeinschaft (Griechenland 1981; Spanien und Portugal 1986) waren die Beziehungen zu den Mittelmeeranrainern nicht immer spannungsfrei; Anpassungen der Mittelmeerabkommen an die neuen Gegebenheiten waren erforderlich. Während sich die finanzielle Zusammenarbeit positiv entwickelte, blieben Enttäuschungen im handels-

${ }^{28}$ Vgl. Köhler, V.: Die Entwicklungspolitik der Europäischen Gemeinschaft, in: Europa-Archiv, Folge 24, 1987, S. 709 f.

${ }^{29} \mathrm{Vgl}$. Schipulle, H.: Die Entwicklungspolitik der Europäischen Gemeinschaft in 1980, (Brüssel) 1981, S. 7 ff.; Ochel, W.: Die Entwicklungsländer in der Weltwirtschaft, a.a.O., S. $226 \mathrm{f}$. 
politischen Bereich nicht aus. ${ }^{30}$ Als Ursachen werden unter anderem angeführt: Die Aufweichung von Präferenzen zugunsten anderer Entwicklungsländer, Protektionismus zugunsten der EG-Industrie sowie die EG-Agrarpolitik, die ohne Rücksicht auf die Interessen der traditionellen Lieferanten etabliert wurde. ${ }^{31}$

In den achtziger Jahren sind neben den Abkommen mit den MittelmeerStaaten auch Kooperationsabkommen mit dem Jemen (1984) sowie den Golfstaaten (1988) geschlossen worden. Dabei bekam der Jemen als erstes Land, welches nicht zu den AKP-Staaten gehört, die Möglichkeit eingeräumt, Ausgleichszahlungen für Exportverluste (Stabex; s. Abschn. 2.5.2) bei der Europäischen Gemeinschaft zu beantragen. Ein spezieller Fonds für Entwicklungsländer, die nicht zu den Unterzeichnern des Lomé-Abkommens gehören, wurde für solche Fälle eingerichtet. ${ }^{32}$

Die besonderen Beziehungen der Europäischen Gemeinschaft zu den AKP-Staaten und den Mittelmeeranrainern drängte die Verbindung mit den Ländern in Asien und Lateinamerika zwangsläufig in den Hintergrund. In den letzten Jahren entwickelte die Gemeinschaft jedoch im Rahmen ihrer globalen Kooperationspolitik ein Instrumentarium für diejenigen Entwicklungsländer, zu denen bisher noch keine besonderen Bezjehungen bestanden. ${ }^{33}$ Für viele von diesen Staaten ist die Europäische Gemeinschaft mittlerweile der wichtigste Geber offizieller Entwicklungs- und humanitärer Hilfe. Schwerpunkt dieser Entwicklungszusammenarbeit ist ein "Allgemeines Präferenzsystem", welches für 127 unabhängige Staaten gilt, tatsächlich aber nur 59 Ländern in Asien und Lateinamerika zugute kommt, da den anderen Entwicklungsländern - AKP-Staaten, Mittelmeeranrainer - diese Präferenzen bereits vertraglich zugesichert waren. Das jährlich an neue Entwicklungen anzupassende Präferenzsystem sieht Zollerleichterungen für alle Industrieprodukte vor sowie für etwa 400 landwirtschaftliche Erzeugnisse.

${ }^{30} \mathrm{Vgl}$. Kommission der EG (Hrsg.): Europa - Dritte Welt .., a.a.O., S. $37 \mathrm{ff}$.

31 Vgl. derselbe: Bericht der Kommission an den Rat über die Sondierungsgespräche mit den Mittelmeer- und den Beitrittsländern, Kom (84) 107 vom 11.5.1984, zit. in: derselbe: Europa - Dritte Welt ..., a.a.O., S. 43.

32 Vgl. derselbe: Europa - Dritte Welt, ..., a.a.O., S. 59 f.

${ }^{33} \mathrm{Vgl}$. Nuscheler, F.: Entwicklungspolitik, a.a.O., S. 308 f. 
Weiterhin sind Vorhaben zur finanziellen und technischen $\mathrm{Zu}$ sammenarbeit vorgesehen, wobei folgende Prioritäten gesetzt werden: Deckung des Nahrungsmittelbedarfs, regionale Integration der Entwicklungsländer und die Überwindung von Krisensituationen.

Diesem entwicklungspolitischen Konzept entsprechend wurden in den achtziger Jahren 11 Kooperationsabkommen geschlossen, darunter mit China, aber auch mit regionalen Vereinigungen wie den ASEAN-Staaten, dem Andenpakt und Mittelamerika. Dabei wurden völlig neue Formen der Zusammenarbeit entwickelt, besonders in den Bereichen regionale Integration, ländliche Entwicklung, Energiewirtschaft.

Seit dem Jahre 1987 kann das in den Lomé-Abkommen etablierte System zum Ausgleich von Exporterlöseinbußen (Stabex) auch von den ärmeren (LDC), den sogenannten Nicht-assoziierten Entwicklungsländern angewendet werden. Aus dem eigens für diese Länder etablierten Fonds wurden rückwirkend für das Jahr 1986 Ausgleichzahlungen in Höhe von über 7 Mio. ECU an Bangladesch, Jemen und Nepal gebilligt. Im Jahre 1987 beanspruchten Haiti sowie erneut Bangladesch und Nepal Mittel aus dem Fonds. Bereits im Jahre 1988 zeigte sich, daß die Mittelaustattung in Höhe von 50 Mio. ECU (für 5 Jahre) nicht ausreicht, berechtigte Forderungen mußten gekürzt werden. ${ }^{34}$

Die Mittel für die Nicht-assoziierten Entwicklungsländer werden im Gegensatz zu den Mitteln für die AKP-Staaten jährlich festgesetzt. In den letzten Jahren flossen etwa 70 Prozent der Mittel nach Asien (i.w. Indien, Bangladesch) und etwa 30 Prozent nach Lateinamerika (i.w. Peru, Bolivien, Guatemala, Nicaragua). Dabei sind über 80 Prozent der Mittel für Vorhaben zur ländlichen Entwicklung zur Verfügung gestellt worden. ${ }^{35}$ Insgesamt wurden für die wirtschaftliche Kooperation der Europäischen

34 Verbert, M.: The Stabex system for Latin America and Asian developing countries, in: The Courier, Nr. 117, September - Oktober 1989, S. 95.

35 Vgl. Kommission der EG (Hrsg.): Europa - Dritte Welt ..., a.a.O., S. 67 ff.; derselbe: Die Europäische Gemeinschaft und Lateinamerika, Luxemburg, Juni/Juli 1986, S. 7 ff.; O.V.: Mit zahlreichen Programmen will die EG Firmen beider Regionen zusammenfuihren, in: Handelsblatt vom 27. Februar 1990. 
Gemeinschaft mit Entwicklungsländern in Lateinamerika und Asien in den Jahren 1976 bis 1988 über 171 Mio. ECU aufgebracht. ${ }^{36}$

Neben den bisher aufgeführten Formen der Entwicklungszusammenarbeit unterstützt die Gemeinschaft auch Entwicklungshilfeprojekte von europäischen Nichtregierungsorganisationen. Im wesentlichen handelt es sich um Kleinvorhaben in ländlichen Regionen von Entwicklungsländern, an denen Vertreter der Organisation selbst aktiv beteiligt sind. Dabei wird das Handeln der EG-Kommission weitgehend durch die Erfahrungen der nichtstaatlichen Organisationen beeinflußt.

Im Jahre 1987 betätigte sich die Gemeinschaft an der Finanzierung von 423 Projekten.

Weiterhin stellt die Europäische Gemeinschaft Nahrungsmittelhilfe im Rahmen der Internationalen Nahrungsmittelhilfe-Übereinkunft aus dem Jahre 1986, Soforthilfe sowie Flüchtlingshilfe zur Verfügung. ${ }^{37}$

Bis Anfang 1988 hatte die Europäische Gemeinschaft insgesamt etwa 14 Mrd. ECU für Vorhaben in Entwicklungsländern zugesagt, davon waren nahezu 12 Mrd. ECU ausgezahlt. ${ }^{38}$

Seit jeher fand auf dem Gebiet der Entwicklungspolitik ein enger Austausch statt zwischen der EG-Kommission, den EG-Mitgliedstaaten, dem Ausschuß für Entwicklungshilfe der OECD (DAC) sowie der Weltbankgruppe und dem Internationalen Währungsfonds. So hat die EG-Kommission in den letzten Jahren unter anderem bei der Suche nach Ansätzen zur Lösung des Schuldenproblems in der Dritten Welt mitgewirkt, an der UNCTAD-Initiative zur rückwirkenden Anpassung der EntwicklungshilfeKonditionen und an der Vorbereitung für den Wirtschaftsgipfel von Vene-

${ }^{36} \mathrm{Vgl}$. Kommission der EG (Hrsg.): Reviewing aid to the Latin American and Asian LLDC's, in: The Courier, Nr. 117, September - Oktober 1989, S. 48 - 49.

${ }^{37} \mathrm{Vgl}$. Bundesministerium für wirtschaftliche Zusammen-arbeit (Hrsg.): Journalistenhandbuch, Entwicklungspolitik '89/90, Bonn 1989, S. 141 f.; Kommission der EG (Hrsg).: Europa - Dritte Welt ..., a.a.O., S. 78 .

${ }^{38}$ Eigene Berechnung aus: Kommission der EG (Hrsg.): Europa - Dritte Welt ..., a.a.O., S. 5 und Bundesministerium für wirtschaftliche $\mathrm{Zu}-$ sammenarbeit (Hrsg.): Journalistenhandbuch ..., a.a.O., S. 137. 
dig im Jahre 1987. Dort wurde die Notwendigkeit einer Sonderaktion hinsichtlich der Schulden der afrikanischen Staaten aufgezeigt.

Die EG-Kommission hat auch zur Festlegung der DAC-Prioritäten für die Entwicklungszusammenarbeit in den neunziger Jahren aktive Beiträge geleistet (Entwicklungshilfe - Koordination, Strukturanpassung). ${ }^{39}$

\subsection{Die Entstehung, Zielsetzungen und Konzepte der Lomé-Ab- kommen}

Das Abkommen von Lomé wurde im Jahre 1975 zum ersten Male zwischen der Europäischen Gemeinschaft und Ländern des afrikanischen, karibischen und pazifischen Raumes - den AKP-Staaten - geschlossen. Bei der Unterzeichnung des vierten Lomé-Abkommens - Lomé IV - im Dezember 1989 war die Zahl der AKP-Staaten auf 69 angewachsen (s. Anhang 1 ).

Die Bevorzugung gewisser Entwicklungsländer seitens der Europäischen Gemeinschaft durch das Einräumen weitgehender Präferenz resultiert aus den kolonialen Verhältnissen, die Mitte der fünfziger Jahre bei den Verhandlungen zur Etablierung der Europäischen Wirtschaftsgemeinschaft (EWG) noch aktuell waren. Von den 6 EWG-Gründungsmitgliedern besaBen Frankreich, Belgien und die Niederlande noch Kolonien, während Italien im Auftrag der Vereinten Nationen das damalige Somaliland verwaltete. Im Teil IV des EWG-Gründungsvertrages vom 20. März 1957, Art. 131 - 136, schlugen sich diese historischen Bindungen von Überseeischen Ländern und Gebieten (ÜLG) an einzelne Gemeinschaftsmitglieder nieder; die mit ihnen verbundenen außereuropäischen Hoheitsgebiete wurden mit der EWG assoziiert.

Besonders Frankreich machte seine Zustimmung zum EWG-Gründungsvertrag von einer Regelung für die ÜLG abhängig. Neben einer Förderung der sozialen und wirtschaftlichen Entwicklung sollten enge Wirt-

${ }^{39}$ Vgl. Kommission der EG (Hrsg.): Europa - Dritte Welt ..., a.a.O., S. 14 und $92 \mathrm{f}$.; Organisation für wirtschaftliche Zusammenarbeit und Entwicklung (Hrsg.): Zusammenarbeit im Dienst der Entwicklung, Bericht 1988, Paris 1988, S. 22 ff. und S. $143 \mathrm{ff}$. 
schaftsbeziehungen geknüpft werden, mit gleichen Handelsbedingungen wie unter den Staaten der Europäischen Wirtschaftsgemeinschaft.

Neben den AKP-Staaten sind auch die ÜLG - sie stehen auch heute noch unter britischer, französischer und niederländischer Verwaltung - mit der Europäischen Gemeinschaft assoziiert. Ein im Jahre 1976 gefaßter Beschluß des europäischen Ministerrates ermögicht es, die entscheidenden Instrumente der Lomé-Abkommen auch bei den ÜLG anzuwenden. Die dazu notwendigen Mittel sind von der Europäischen Gemeinschaft über die Europäischen Entwicklungsfonds bereitgestellt worden, sie gehören jedoch nicht in den Rahmen der Lomé-Abkommen. Diese Beträge haben sich während der Laufzeit der jeweiligen Lomé-Abkommen verringert, da sämtliche ÜLG, die unabhängig geworden waren, den Lomé-Abkommen beigetreten sind, worauf die vorgesehenen Mittel in die AKP-Titel übertragen wurden. ${ }^{40}$

Trotz des EG-Beitritts von Spanien und Portugal sind von den ehemaligen Kolonien dieser jüngsten EG-Mitglieder keine Beitrittswünsche zu dem Lomé-Abkommen laut geworden. Dies resultiert aus der Tatsache, daß die entsprechenden Entwicklungsländer die Abkommen bereits unterzeichnet haben beziehungsweise, da sie die geographischen Voraussetzungen für einen Beitritt zu den Lomé-Abkommen nicht erfüllen, durch andere Verträge mit der Europäischen Gemeinschaft assoziiert sind.

Schon bald nach der Gründung der Europäischen Gemeinschaft forderte die Phase der Entkolonialisierung eine Neugestaltung der im Teil IV des Gründungsvertrages geregelten Beziehungen. Als Anpassung an die veränderten außenpolitischen Realitäten schloB die Europäische Gemeinschaft mit 18 unabhängig gewordenen afrikanischen Staaten und Madagaskar (AASM Staaten) einen Assoziierungsvertrag - das Abkommen von Jaunde, welches im Jahre 1964 für $5 \mathrm{~J}$ ahre in Kraft trat. Bei diesem Vertrag und dem Folgeabkommen (Jaunde II vom 1. Januar 1971 bis zum 31. Januar 1975) ging es im wesentlichen um einen beiderseitigen freien Marktzutritt und um ein Hilfsprogramm der Europäischen Gemeinschaft für die

${ }^{40}$ Vgl. Schipulle, H.: Die Entwicklungspolitik der Europäischen Gemeinschaft, a.a.O., S. 34. 
AASM-Staaten, welches durch den ersten bis dritten Europäischen Entwicklungsfonds (EEF) finanziert wurde. ${ }^{41}$

Gleichzeitig mit Jaunde II wurde noch ein weiterer Assoziationsvertrag mit 3 ostafrikanischen Staaten - später kam noch Mauritius hinzu - geschlossen: das Abkommen von Arusha. Eine absolute Gleichstellung mit den "Altassoziierten" von Jaunde ist jedoch bewußt unterlassen worden, da deren Position sonst geschwächt worden wäre. ${ }^{42}$

Das auslösende Element für die Verhandlungen zum Lomé-I-Abkommen war der EG-Beitritt Großbritanniens im Jahre 1973. Die bisherige Entwicklungspolitik der Europäischen Gemeinschaft mit ihrem Schwerpunkt im frankophonen Afrika war für Großbritannien - wegen seiner Beziehungen zum Commonwealth - nicht akzeptabel. ${ }^{43}$

Zusätzlich formulierten in den Jahren 1974 und 1975 die Entwicklungsländer auf der sechsten und siebten UN-Sondergeneralversammlung Forderungen zur Errichtung einer Neuen Weltwirtschaftsordnung. Nach gut einem Jahrzehnt der Selbständigkeit hatten sich die erwarteten Erfolge einer eigenen Entwicklung immer noch nicht eingestellt, zum Beispiel hatten die starken Exportabhängigkeiten von nur wenigen Rohstoffen weiterhin Bestand.

Aus dieser Situation heraus war die Europäische Gemeinschaft zu einigen Zugeständnissen bereit, zumal außerdem ein großes Interesse bestand, die Sicherung der Exportgütermärkte und der Rohstoffversorgung - auch im Hinblick auf amerikanische und japanische Konkurrenten - auszubauen.

So wurden von November 1973 bis Januar 1975 Verhandlungen geführt, die trotz der 55 Teilnehmerstaaten formal als bilateral anzusehen waren. Die Erarbeitung einer gemeinsamen Verhandlungsbasis stellte sich sowohl bei der Europäischen Gemeinschaft als auch bei den damals 46 Staaten

$41 \mathrm{Vgl}$. Wawrzik, K.: Bericht im Namen des Ausschusses für Entwicklung und Zusammenarbeit, 17. November 1980, S. 19; Glaser, T.: EECACP cooperation: the historical perspective, in: The Courier, Nr. 120, März - April 1990, S. 26.

${ }^{42}$ Das Abkommen von Arusha sah keine finanzielle und technische Hilfe vor.

$43 \mathrm{Vgl}$. Krohn, H.B.: Das Abkommen von Lomé (I) zwischen der EG und den AKP-Staaten, in: Europa-Archiv, Folge 6, 1975, S. 177. 
Afrikas, der Karibik und des Pazifiks (AKP-Staaten ${ }^{44}$ ) als problematisch heraus. ${ }^{45}$ Die Komplexität der zu lösenden Schwierigkeiten war sowohl durch die unterschiedlichen politischen, wirtschaftlichen und geographischen Verhältnisse bedingt, als auch durch die verschiedensten Auffassungen über die Inhalte der Konvention. ${ }^{46}$

Infolge der verhandlungstaktischen Geschicklichkeit - besonders der Commonwealth-Länder - und der Option der Europäer, den Partnern sehr weitgehendes Entgegenkommen zu zeigen, ${ }^{47}$ befand sich die Gemeinschaft gegenüber den Forderungen der AKP-Staaten meistens in der Defensive. Die Unterzeichnung des Lomé-I-Abkommens erfolgte am 28. Februar 1975. Auf die Schwerpunkte der Abkommen von Lomé wird im Kapitel 2.5 näher eingegangen.

Das zweite AKP-EG-Abkommen von Lomé ist am 31. Oktober 1979 unterzeichnet worden und trat am 1. Januar 1981 in Kraft, um so das am 1. März 1980 ausgelaufene Lomé-I-Abkommen zu ersetzen. Neben den 10 EG-Mitgliedstaaten ratifizierten letztlich 64 Entwicklungsländer das Lome-II-Abkommen.

Die teilweise recht kontrovers geführten Verhandlungen begannen - wie in Artikel 91 des Lomé-I-Abkommens vorgesehen - 18 Monate vor Ablauf von Lomé I.

Nach der grundsätzlich positiven Einschätzung der Erfahrungen mit dem ersten Abkommen war die Europäische Gemeinschaft bemüht, eine Fortführung von Lomé I mit einer Anpassung an die neuen Gegebenheiten zu

4419 altassoziierte Länder (AASM), 21 Commonwealth-Staaten und 6 weitere afrikanische Länder.

${ }^{45}$ Vgl. Krohn, H.B.: Das Abkommen von Lomé (I) ..., a.a.O., S. 179.

${ }^{46}$ Vgl. O.V.: Abkommen AKP-EWG von Lomé (I), in: Europa-Archiv, Dokumente, Folge 6, 1975, S. D 165.

${ }^{47}$ Festgelegt durch das Protokoll 22 im Anhang der Beitrittsakte; Vgl. Wawrzik, K.: Bericht ..., a.a.O., S. 20 f. 
erreichen. Die AKP-Staaten dagegen verlangten radikale Änderungen, durch die ihre Zukunft gesichert werden sollte. ${ }^{48}$

Wiederum blieb die Europäische Gemeinschaft während der Lomé-II-Verhandlungen in der Defensive, da sich für sie nur die Frage stellte, inwieweit die Forderungen der AKP-Staaten "angesichts ihrer eigenen Möglichkeiten und Grenzen akzeptabel waren".49 Trotz erheblicher Zugeständnisse seitens der Europãer ${ }^{50}$ beklagte die AKP-Seite nach Abschluß der Verhandlungen, daß sie zu viele Kompromisse habe schließen müssen und daß die Aufstockung der Finanzmittel unangemessen niedrig sei. ${ }^{51}$ Ein Autor spricht sogar von einem den AKP-Staaten aufgedrückten Partnerschaftskonzept, welches kaum dazu beitrüge, die Struktur der Abhängigkeit zu verbessern. ${ }^{52}$ Die Enttäuschung, die sich im AKP-Lager über das vermeintlich zu geringe Entgegenkommen der Europäischen Gemeinschaft breit machte, kam bei der Abstimmung über die Annahme des Lomé-II-Abkommens zum Ausdruck. Es ist nur eine knappe Mehrheit von $26 \mathrm{zu} 22$ Stimmen und mehreren Enthaltungen zugunsten des Lomé-II-Abkommens erreicht worden. ${ }^{53}$

Einer der Gründe für die Unzufriedenheit war, daß sich die Lage der AKP-Staaten trotz Lomé I verschlechtert hatte. Die finanziellen Mittel waren nur bedingt in der Lage, die Folgen der wirtschaftlichen Probleme in den AKP-Staaten zu mildern, behoben werden konnten sie nicht. Hinzu kam, daß die positiven Auswirkungen des Abkommens überschätzt wur-

$48 \mathrm{Vgl}$. Bundesrat (Hrsg.): Entwurf eines Gesetzes zu dem Zweiten AKPEWG-Abkommen von Lomé vom 31. Oktober 1979, Bundesratsdrucksache 160/80, Bonn 1980, S. 53; Meyer, K.: Die zweite Konvention von Lomé, in: Europa-Archiv, Folge 1, 1980, S. 11; Asante, S.K.B.: Lomé-Il: Alte Abhängigkeit im neuen Gewand?, in: Entwicklung und Zusammenarbeit, Vol. 22, Oktober 1981, S. 7.

${ }^{49} \mathrm{Vgl}$. Meyer, K.: Die zweite Konvention ..., a.a.O., S. 12.

so Vgl. Bundesrat (Hrsg.): Entwurf eines Gesetzes ..., a.a.O., 1980, S. 53.

51 Vgl. Strizek, H.: Die neue AKP-EWG-Konvention (Lomé II), in: Entwicklungspolitik, Materialien Nr. 66, Bonn 1980, S. 6; St. John, B.: "The groundwork of our continued cooperation in the 1980s", in: The Courier, Nr. 58, November 1979, S. 5.

52 Vgl. Asante, S.K.B.: Lomé-II ..., a.a.O., S. 7.

${ }^{53} \mathrm{Vgl}$. Twitchett, C.C.: Lomé II: die Enttäuschungen der AKP-Länder, in: Europa-Archiv, Folge 3, 1980, S. 83. 
den $^{54}$ und die dadurch hochgesteckten Erwartungen nicht erfüllt werden konnten.

Trotz des europäischen Entgegenkommens waren auch seitens der Europäer kritische Stimmen zu vernehmen. So wurde in einer Untersuchung Anfang der achtziger Jahre festgestellt, daß die Öffnung der EG-Märkte allein nicht genüge, um den für die AKP-Staaten so wichtigen Handel mit der Europäischen Gemeinschaft zu aktivieren. ${ }^{55}$

Aber eine wesentliche Steigerung der finanziellen Leistungen war für die Europäische Gemeinschaft nicht akzeptabel, vor allem mit Rücksicht auf Verpflichtungen aus den Assoziierungsverträgen mit den Mittelmeeranrainern und den weltweiten Kooperationsabkommen. ${ }^{56}$

Die Alternative zu einer drastischen Mittelaufstockung wurde bei den Nachfolge-Abkommen in einer verbesserten Qualität der Kooperationsmöglichkeiten gesucht. Dieses Bestreben läßt sich bereits in der Strukturierung des Lome-III-Abkommens erkennen, wo die einzelnen Instrumente der Kooperation deutlich herausgearbeitet und im dritten Teil zusammengefaßt wurden. Dies aber bedeutete auch eine Detaillierung und Spezialisierung der Maßnahmen, wodurch eine Vergrößerung des Verwaltungsapparates und eine Ausweitung der Kontrollmaßnahmen nicht zu vermeiden war.

Das Dritte Abkommen von Lomé ist am 8. Dezember 1984 unterzeichnet worden und trat am 1. Mai 1986 in Kraft, nachdem es von den EG-Mitgliedstaaten und 44 AKP-Staaten (erforderliche 2/3-Mehrkeit) ratifiziert worden war.

${ }^{54}$ Vgl. Helldorf, K.v.: Die Abkommen von Lomé: Immer noch Wegweiser?, in: Entwicklung und Zusammenarbeit, Vol. 22, Oktober 1981, S. 9.

${ }^{55}$ Vgl. Focke, K.: Von Lomé 1 zu Lomé 2, Luxemburg 1980, S. 16.

${ }^{56} \mathrm{Vgl}$. Claus, B.: Lomé II, Verhandlungspositionen und Ergebnisse, in: GTZ (Hrsg.): Technische Zusammenarbeit in der Entwicklungsplanung, Dokumentation einer Fachtagung vom 24. Nov. bis 5. Dez. 1979, Wölfersheim, S. 160; Cheysson, C.: "Our experiment has been converted into a policy, the Lomé-policy", in: The Courier, Nr. 58, November 1979, S. 9. 
Nach intensiven, zeitweilig sehr kontrovers - auch innerhalb der EGGruppe - geführten, 13-monatigen Verhandlungen ${ }^{57}$ konnte das Vierte Lomé-Abkommen am 15. Dezember 1989 unterzeichnet werden. Am 1. März 1990 traten Übergangsregelungen in Kraft, die die Kontinuität der Lomé-Zusammenarbeit bis zur vollständigen Inkraftsetzung von Lomé IV nach der erforderlichen Ratifizierung durch die 12 EG-Staaten und zwei Drittel der AKP-Staaten gewährleistet. ${ }^{58}$ Das Lomé-IV-Abkommen ist am 1. September 1991 in Kraft getreten. ${ }^{59}$

Das Lome-IV-Abkommen ist - wie bereits die vorherigen Abkommen gekennzeichnet durch die aktuelle Situation der wirtschaftlichen und politischen Verhältnisse in den Unterzeichnerstaaten.

Während sich in Lomé I und Lomé II noch die euphorische Stimmung widerspiegelt, die ehemaligen Kolonien wurden nunmehr als Partner anerkannt und es herrschte die Meinung, daß allein einige "Anschubhilfen" ausreichen würden, um in den AXP-Staaten florierende Wirtschaften zu etablieren, dokumentiert sich in den Nachfolgeabkommen Lomé III und Lome IV ein wesentlich differenzierteres Bild.

Besonders das wirtschaftliche Umfeld hatte sich mittlerweile gewandelt. So konnten noch bis Anfang der achtziger Jahre die AKP-Staaten der Europäischen Gemeinschaft beziehungsweise den Mitgliedstaaten vorhalten, daß dort aufgrund rezessiver Märkte nur reduzierte Volumina von AKPProdukten importiert würden. Seit dem Jahre 1983 jedoch hat sich in den Industriestaaten eine bis zum Ende der achtziger Jahre stetig verlaufende Aufschwungphase herausgebildet, so daß die obige Argumentation der AKP-Staaten nicht verfängt, um die unbefriedigende wirtschaftliche Situation in den meisten AKP-Staaten zu erklären. Die stetige Verschlechterung der wirtschaftlichen Verhältnisse in den AKP-Staaten in den achtzi-

$57 \mathrm{Vgl}$. Oyowe, A.: A convention for a changing world, a.a.O., S. 4; O.V.: AKP und EG ïber Hilfe einjg, in: Blick durch die Wirtschaft, 7. Dezember 1989; O.V.: Zahl der AKP-Staaten soll erhöht werden, in: Frankfurter Allgemeine Zeitung, 29. November 1989; O.V.: Italien und Griechenland blockieren die Verhandlungen mit den AKP-Staaten, in: Handelsblatt, 19. Juli 1989.

$58 \mathrm{Vgl}$. Kommission der EG (Hrsg.): Mitteilungen an die Presse, IP (90) 467, 13. Juni 1990.

59 Vgl. O.V.: Möglichkeiten für europäische Unternehmen im Rahmen von Lomé, in: Blick durch die Wirtschaft, 1. Januar 1992. 
ger Jahren war zum einen durch den dramatischen Verfall der Rohstoffpreise bedingt, zum anderen durch die Schuldenkrise der Dritten Welt. ${ }^{60}$

Dieser Entwicklung wurde in den Lome-Abkommen durch eine deutliche Schwerpunktverschiebung Rechnung getragen. Dies wird zum Beispiel daran deutlich, daß sowohl ein erheblicher Teil der ursprünglich als Sonderdarlehen ausgezahiten Mittel nunmehr in Zuschüsse umgewandelt wurde, als auch die Rohstoffsicherungskomponenten bei den Verfahren Sysmin und Stabex völlig in den Hintergrund getreten sind (s. Kap. 2.6 und 4.8).

Bei den Verhandlungen zum Lomé-I-Abkommen verpflichtete sich die Europäische Gemeinschaft, die neuen AKP-Staaten nicht schlechter zu stellen als die AASM-Staaten (19 ehemalig Assoziierte Afrikanische Staaten und Madagaskar, die Unterzeichner des Jaunde-Abkommens). Der AKP-Forderung nach $8 \mathrm{Mrd}$. ECU jedoch konnte die Gemeinschaft bei weitem nicht entsprechen. Das Angebot von 3,4 Mrd. ECU - davon 40 Mio. ECU Darlehen der EIB - mußte schließlich von den AKP-Staaten akzeptiert werden, wobei jedoch der Beginn der Zahlungen rückwirkend zum 28. Februar 1975 angesetzt wurde. ${ }^{6 t}$

Für die fünfjährige Laufzeit der Lomé-II-Konvention wurden insgesamt etwa 5,6 Mrd. ECU bereitgestellt. Rechnet man 10 Mio. ECU zugunsten der Überseeischen Länder und Gebiete (ÜLG) Frankreichs, der Niederlande und des Vereinigten Königreiches hinzu, bedeutete dies eine Steigerung von etwa 60 Prozent im Vergleich zum Lomé-I-Abkommen. ${ }^{62}$

Gleichwohl konnten diese Aufwendungen angesichts der AKP-Probleme bereits im Jahre 1980 betrug die Staatsverschuldung allein der afrikanischen AKP-Staaten rund 30 Mrd. ECU ${ }^{63}$ - nur als Tropfen auf dem heißen Stein angesehen werden. Dabei ist zu berücksichtigen, daß durch den inflationsbedingten Kaufkraftschwund der EG-Währungen und die Erweite-

${ }^{60} \mathrm{Vgl}$. Oyowe, A.: A convention for a changing world, a.a.O., S. 3.

${ }^{61}$ Vgl. Krohn, H.B.: Das Abkommen von Lomé (I) ..., a.a.O., S. 185 f.

${ }^{62} \mathrm{Vgl}$. Bundesrat (Hrsg.): Entwurf eines Gesetzes ..., a.a.O., 1980, S. 60 .

${ }^{63}$ Vgl. Focke, K.: Von Lomé 1 zu Lomé 2, a.a.O., S. 71. 
rung der AKP-Staaten die Aufstockung der Mittel keine reale Verbesserung für die AKP-Staaten darstellte. ${ }^{64}$

Auch die Erhöhung der Mittel für Lomé III auf 8,5 Mrd. ECU entsprach nicht der AKP-Forderung, die eine Verdoppelung des Lomé-II-Etats vorsah. Diese Forderung, sie erscheint angesichts der respektablen Höhe der bereitgestellten Mittel unangemessen, kann relativiert werden, wenn dem Betrag andere Ausgaben der Europäischen Gemeinschaft gegenübergestellt werden. So betrugen allein die Ausgaben im Rahmen des landwirtschaftlichen Struktur- und Garantiefonds für Milch und Milcherzeugnisse im Jahre 1983 etwa 4,4 Mrd. ECU, im Jahre 1986 bereits 6,6 Mrd. ECU. ${ }^{65}$ Die von der Europäischen Gemeinschaft im Rahmen des VII. Europäischen Entwicklungsfonds (EEF) sowie von der Europäischen Investitionsbank für Lomé IV zur Verfügung gestellten Mittel betragen 12 Mrd. ECU (s. Tab. 1); hinzukommen noch Mittel in Höhe von 165 Mio. ECU für die ÜLG. Dabei ist anzumerken, daß auch der VII. EEF genau wie die vorherigen Fonds nur eine fünfjährige Laufzeit hat, wohingegen erstmals für das Lomé-IV-Abkommen ein Vertragsdauer von zehn Jahren vereinbart wurde.

Die Gesamtsumme, welche für das Lome-IV-Abkommen (Lomé III) zur Verfügung gestellt wurde, läßt sich nach der Mittelbereitstellung in folgende Gruppen unterteilen:

- 10.800 (7.400) Mio. ECU für den VII. (VI.) Europäischen Entwicklungsfonds (EFF). Davon sind 1.500 (925) Mio. ECU für das Stabex-System und 480 (415) Mio. ECU für die Sysmin-Regelung vorbehalten. Für den neu etablierten Strukturanpassungsfonds sind 1.150 Mio. ECU reserviert.

- 1.200 (1.100) Mio. ECU zinsvergünstigte Darlehen aus den eigenen Mitteln der Europäischen Investitionsbank (EIB), die in der Regel rentablen Vorhaben in den Bereichen Industrie, Bergbau und Tourismus vorbehalten bleiben.

${ }^{64}$ Vgl. Tetzlaff, R.: Lomé II: "Nachgeschmack von Frustration", in: epdEntwicklungspolitik 4/80, S. 12; St. John, B.: "The groundwork ..., a.a.O., S. 5.

${ }^{65} \mathrm{Vgl}$. O.V.: Die EG-Agrarausgaben im vergangenen Jahr, in: Neue Zürcher Zeitung, 10. Oktober 1984; Cherrington, J.: The EEC's dairy desperation, in: Financial Times, 18. November 1986. 
Tabelle 1: Dle flnanziellen Aufwendungen der Europäischen Gemeinschaft zugunsten der AKP-Staaten

\begin{tabular}{|c|c|c|c|c|c|}
\hline & $\begin{aligned} \text { Lomé } & \\
1976 & -1980\end{aligned}$ & $\begin{array}{c}\text { Lomé II } \\
1981-1985\end{array}$ & $\begin{array}{c}\text { Lomé III } \\
1985-1990\end{array}$ & $\begin{array}{l}\text { Lon } \\
1990\end{array}$ & $\begin{array}{l}\text { mé IV } \\
-1995\end{array}$ \\
\hline Gesamt & 3373 & 5530 & 8500 & 12 & 000 \\
\hline \multicolumn{6}{|l|}{ Europäische Entwicklungsfonds } \\
\hline Darlehen & 2058 & 2998 & 4860 & 7 & $995 *$ \\
\hline Sonderdarlehen & 446 & 524 & 600 & & - \\
\hline Rislkokapital & 97 & 284 & 600 & & 825 \\
\hline stabex & 382 & 557 & 925 & 1 & 500 \\
\hline Sysmin & - & 282 & 415 & & 480 \\
\hline \multicolumn{6}{|l|}{ Europäische Invest1t1onsbank } \\
\hline Darlehen mit ermäß1gtem zins & 390 & 685 & 1100 & 1 & 200 \\
\hline $\begin{array}{l}\text { Darlehen auf dem Bergbau- und } \\
\text { Energie-Sektor (bei beidersei- } \\
\text { tigem Interesse) }\end{array}$ & - & 200 & - & & - \\
\hline
\end{tabular}

Anm.: Angaben gerundet; in Lomé I zusätzlich + 88 Mio. ECU als Unterhaltskosten für die EGVertretungen in den AKP-Staaten

*) Davon 1150 Mlo. ECU für den strukturanpassungsfonds.

Quelle : Eigene zusammenstellung nach: Europäische Investitionsbank (Hrsg.): 25 Jahre 1958 - 1983 , o.0., Apr11 1983, S. 80; Kommission der EG (Hrsg.): Lomé-III, Europe-Information: Development, Brüssel, Dezember 1984, Anhang 6; 0.V.: Detalls on principal developments and Innovations, in: The Courfer, Nr. 120, März - April 1990, S. $14 \mathrm{f}$. 
Aus den Lomé-Abkommen, wo im Jahre 1975 erstmals der Versuch unternommen wurde, in einem einheitlichen Vertragswerk zwischen einer Gruppe von Industrie- und Entwicklungsländern alle Bereiche, die für die Nord-Süd-Zusammenarbeit von Relevanz sind, zu erfassen, erwachsen den Vertragspartnern Rechte und Pflichten. ${ }^{66}$ Letztere sind allerdings nicht immer paritätisch ausgewogen. Als Beispiel sei hier der Verzicht der Gemeinschaft auf Reziprozität im Handelsverkehr aufgeführt. Hier kommt deutlich die entwicklungspolitische Komponente der Lomé-Abkommen zum Ausdruck. Die Aussage, "die AKP-Staaten fordern, die Europäische Gemeinschaft zahlt", ${ }^{67}$ erscheint etwas plakativ formuliert zu sein, trifft im Kern jedoch durchaus zu. Insgesamt können die Lome-Abkommen als "kombinierte Wirtschafts-, Entwicklungs- und Handelsverträge mit politischen Akzenten" bezeichnet werden. ${ }^{68}$

Wie in den verschiedenen Verhandlungen zu den einzelnen Abkommen deutlich wurde, sind die Vertragspartner stets mit unterschiedlichen, teilweise konträren Zielvorstellungen an den Verhandlungstisch gegangen. Abbildung 1 soll dies mit einer Darstellung der unterschiedlichen Schwerpunkte für den Bergbausektor veranschaulichen.

Obwohl dieses Modell nicht auf alle Verknüpfungen und Komponenten der Realität eingehen kann, genügt es dem Anspruch, schematisch die verschiedenen Erwartungen und möglichen Ziele der Lomé-Partner aufzuzeigen:

Die EG-Mitgliedsstaaten waren bereit, die Zusammenarbeit mit den AKPStaaten auf Basis der Lomé-Abkommen auszubauen und sehen darüberhinaus in den Vereinbarungen eine Möglichkeit zur Realisierung ihrer Rohstoffpolitik. Die internationale Rohstoffpolitik rohstoffabhängiger

66 Vgl. Köhler, V.: Die Entwicklungspolitik der Europäischen Gemeinschaft, a.a.O., S. 710.

${ }^{67}$ Persönliches Gespräch, Bundeswirtschaftsministerium Bonn.

${ }^{68}$ Der Begriff "Assoziierung" war von den AKP-Staaten als diskriminierend empfunden worden; vgl. Wawrzik, K.: Bericht ..., a.a.O., S. 24. 
Industrieländer ${ }^{69}$ - aber auch der Europäischen Gemeinschaft - läßt sich unter anderem in folgende Komponenten aufgliedern:

- Sicherung der Rohstoffversorgung durch Absprachen auf politischer Ebene und vertragliche Vereinbarungen;

- Zusammenarbeit bei der Suche, Gewinnung und Verarbeitung von Rohstoffen mit dem Ausland;

- Unterstützung der nationalen Industrie bei Bergbauvorhaben im Ausland;

- Technologietransfer;

- Reduktion der Belastungen von Mensch und Umwelt durch Bergbauvorhaben.

Wie aus der Auflistung ersichtlich wird, kann zwischen den einzelnen Zielen nicht immer eine scharfe Trennung vorgenommen werden, da zum Teil Überschneidungen auftreten. Daher wird im Rahmen dieser Arbeit nur auf zwei Hauptziele der Europäischen Gemeinschaft im Hinblick auf die Zusammenarbeit mit den AKP-Staaten eingegangen: Sicherung der Rohstoffversorgung sowie Aspekte der Entwicklungszusammenarbeit. Da auch bei dieser Zweiteilung noch Überschneidungen möglich sind, wird sofern eine Differenzierung notwendig ist - das Primärziel für eine $\mathrm{Zu}$ ordnung ausschlaggebend sein.

Im Verlaufe dieser Arbeit wird deutlich, daß bei den jeweiligen Verhandlungen zur Sysmin-Regelung die Sicherung der EG-Rohstoffversorgung nicht im Vordergrund gestanden hat. Dennoch waren in den ersten Versionen des Sysmin-Verfahrens - wie auch in den gesamten Lomé-Abkommen - deutlich rohstoffpolitische Ziele enthalten, die zwar primär der entwicklungspolitischen Komponente zuzuordnen sind, aber sekundär auch versorgungspolitische Belange der Gemeinschaft berühren (s. Abb. 1). In Lomé IV jedoch ist diese Komponente noch stärker in den Hintergrund getreten.

${ }^{69}$ Vgl. Gocht, W.R.; Zantop, H.; Eggert, R.G.: International mineral economics, Berlin/Heidelberg/New York/Tokyo 1988, S. 219 f.; Gocht, W.: Wirtschaftsgeologie und Rohstoffpolitik, 2. Aufl., Berlin/ Heidelberg/New York/Tokyo 1983, S. 204 f. 
Abbildung 1:Die unterschiedlichen Zielvorstellungen der Lomé-Vertragspartner auf dem Bergbausektor

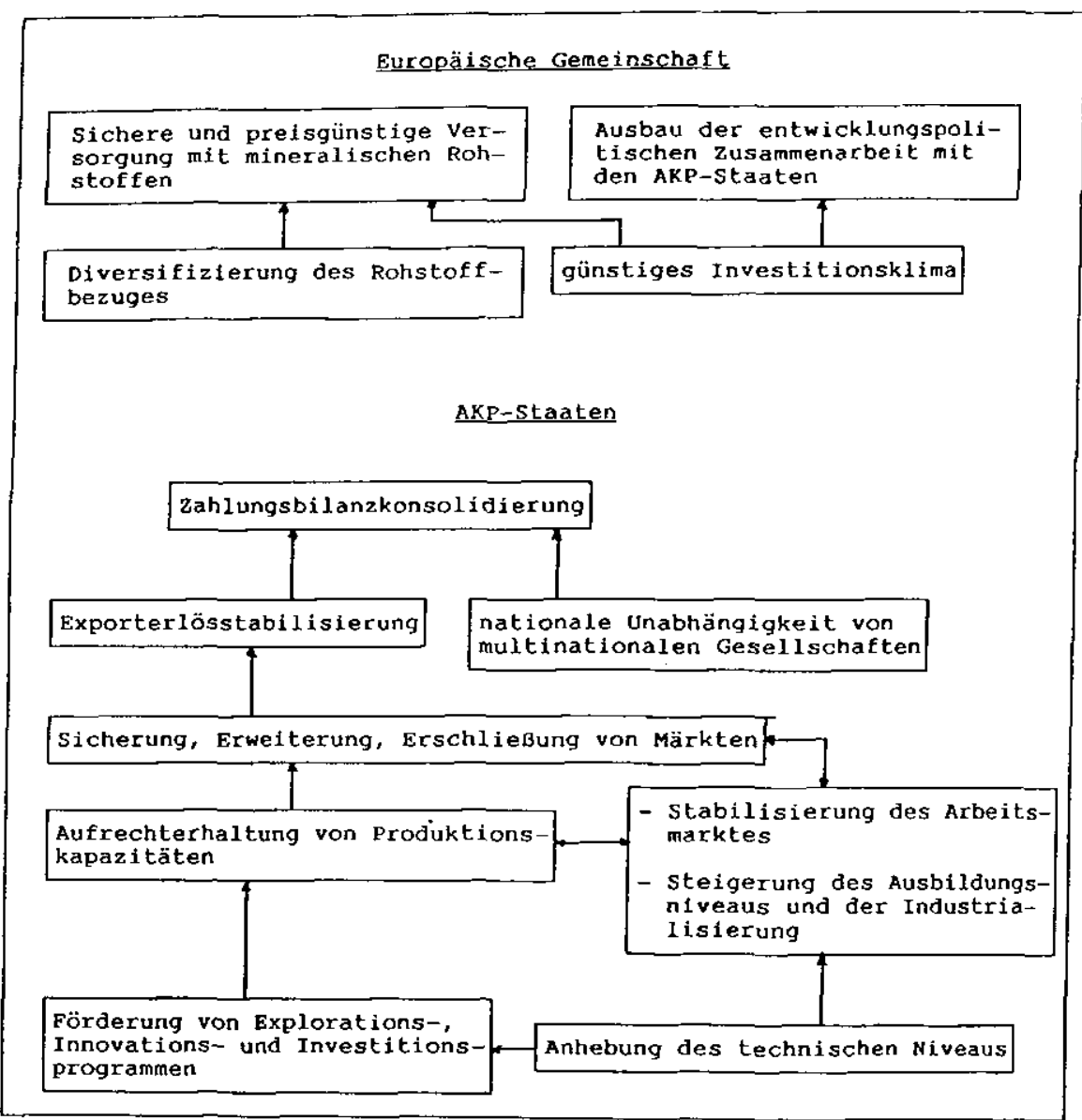

Quelle: Eigener Entwurf. 
Die AKP-Staaten dagegen haben möglicherweise erwartet, mit Hilfe der Lomé-Abkommen unter anderem der Konsolidierung ihrer Zahlungsbilanzen näherzukommen. Nach ihren Vorstellungen wäre dies bei stabilen Erlösen für ihre Exporte sowie durch eine stärkere Unabhängigkeit von ausländischen Gesellschaften, da auf diese Weise der Kapitalabfluß ins Ausland vermindert wäre, erreicht worden.

Zur Erlösstabilisierung kann auch eine Erweiterung bestehender und Erschließung neuer Absatzmärkte beitragen; dazu sind jedoch intakte Produktionskapazitäten unabdingbar. Diese Kapazitäten sind unter anderem nur aufrechtzuerhalten, wenn stabile Arbeitsmarktbedingungen herrschen und das Ausbildungsniveau gesteigert wird. Staatliche Förderprogramme könnten auf die diversen Aktivitäten stimulierend wirken.

Neben divergierenden Zielvorstellungen bei den jeweiligen Lomé-Unterzeichnern lassen sich auch gemeinsame Interessen aufzeigen, wobei die Vertragspartner jedoch häufig unterschiedliche Gewichtungen vornehmen. Anhand einer Aufstellung der Interessen wird deutlich, daß auch den Europāern an den Lome-Abkommen gelegen ist - nicht nur aus entwicklungspolitischen Gründen (s. Abb. 2). Davon unberührt bleibt, daß mit Ausnahme der Entwicklungszusammenarbeit die Interessen der Europäer in den Vertragswerken Lomé III und Lomé IV immer weniger berücksichtigt werden.

Das generell stark ausgeprägte Interesse der AKP-Staaten an den LoméAbkommen ist evident. Es erklärt sich aus der Tatsache, daß die LoméVerträge direkt auf die Bedürfnisse der AKP-Staaten zugeschnitten wurden. Weiterhin resultiert das vehemente Interesse der AKP-Länder an den einzelnen Instrumenten der Lomé-Abkommen (s. Abb. 2) aus der - zwischen den AKP-Staaten und der Europäischen Gemeinschaft - unterschiedlichen Handelsstruktur. Während für die Europäische Gemeinschaft der Handel mit den AKP-Ländern nur eine zweitrangige Rolle spielt, da der Warenaustausch mit den Industrieländern dominiert, wickeln die AKPStaaten einen Großteil ihres Handels mit der Europäischen Gemeinschaft beziehungsweise den einzelnen Mitgliedstaaten ab. So wurden im Jahre 1983 trotz rückläufiger Tendenz über 35 Prozent der AKP-Einfuhren (Warenwert) aus der Europäischen Gemeinschaft importiert, ${ }^{70}$ die Europäische

${ }^{70} \mathrm{Vgl}$. Statistisches Amt der EG (Hrsg.): Foreign trade: third countries, statistical yearbook 1977 - 1983, Vol. A, ACP-Countries, Luxemburg 1986, S. 26. 
Abbildung 2: Die Qual1fizierung der unterschiedlichen Interessen an den Lomé-Abkommen

\begin{tabular}{|c|c|c|c|c|}
\hline & stark & rege & mäßig & gering \\
\hline $\begin{array}{l}\text { Erlösstabilisierung } \\
\text { Erhöhung der Deviseneinnahmen } \\
\text { Steigerung des volkselnkommens in den AKP-Staaten } \\
\text { Diversifizierung der produktion agrartscher und } \\
\text { mineralischer Rohstoffe } \\
\text { Sicherung der versorgung mit mineralischen Rohstoffen } \\
\text { Sicherung der versorgung mit agrarischen Rohstoffen } \\
\text { Prelsstabilisierung bei Rohstoff-Importen der EG } \\
\text { Infrastrukturverbesserung in den AKP-Staaten } \\
\text { Industrialisierung in den AKP-Staaten } \\
\text { Arbeitsplatzschaffung/-sicherung in den AKP-Staaten } \\
\text { Know-How-Transfer } \\
\text { Reduzierung der Abhängigkeiten von multinationalen } \\
\text { Bergbaugeselischaften nicht europäischen Ursprungs } \\
\text { Vermeiden von Konkurrenz bei Halb- und Fertigprodukten } \\
\text { Investitionsschutz } \\
\text { Schaffung und Ausweitung von Märkten } \\
\text { Entwickiungszusammenarbeit }\end{array}$ & $\begin{array}{l}+ \\
+ \\
+ \\
0 \\
+ \\
+0\end{array}$ & $\begin{array}{l}0 \\
0 \\
0\end{array}$ & 0 & 0 \\
\hline
\end{tabular}

Anm.: AKP-Staaten $(+)$, EG $(0)$, nähere Erläuterungen im Text

Quelle: Elgener Entwurf. 
Gemeinschaft dagegen bezog im Jahre 1984 weniger als 5 Prozent ihrer Gesamteinfuhr aus den AKP-Staaten. ${ }^{71}$

Das starke Interesse der AKP-Staaten an den rein finanziellen Aspekten der Lomé-Abkommen (Erlösstabilisierung, Erhöhung der Deviseneinnahmen, Steigerung des Volkseinkommens) resultiert aus dem erheblichen Finanzbedarf dieser Länder.

In Entwicklungsländern werden Staatseinnahmen in der Hauptsache - speziell die dringend benötigten Devisen harter Währungen - aus den Erlösen exportierter Waren bezogen (s. Kap. 3.2).

Nur mit einer Steigerung der Exporterlöse läßt sich "Entwicklung" importieren, ${ }^{72}$ ist es möglich, den Schuldendienst zu leisten, erforderliche Importe zu realisieren und notwendige Infrastrukturmaßnahmen durchzuführen. Insofern sind die Mittel, welche derartige Aktionen unterstützen beziehungsweise fördern, bei den AKP-Staaten hoch willkommen, zumal sie zu sehr günstigen Konditionen bereitgestellt werden.

Anders verhält es sich bei der Europäischen Gemeinschaft. Abgesehen von entwicklungspolitischen Überlegungen spielt es für die europäischen Staaten keine gravierende Rolle, ob ein AKP-Land über einen Zeitabschnitt hinaus konstante Erlöse für seine Exportprodukte erzielt oder unter Schwankungen der Preise oder der Mengen seiner Exportgüter zu leiden hat.

Ein etwas stärkeres Interesse kann der Europäischen Gemeinschaft an steigenden Deviseneinnahmen bzw. Volkseinkommen in den AKP-Staaten unterstellt werden.

Neben den entwicklungspolitischen Aspekten sind in diesem Fall die Erwartungen im Hinblick auf die Erschließung zusätzlicher Exportmärkte für die EG-Staaten zu berücksichtigen.

Das Interesse beider Vertragsgruppen an einer Diversifizierung der AKPIndustrie beruht auf der Überlegung, den Export auf eine Vielzahl von

${ }^{11}$ Vgl. Statistisches Amt der EG (Hrsg.): ACP basic statistics 1986, Luxemburg 1986, S. 55.

$72 \mathrm{Vgl}$. Hemmer, H.R. Wirtschaftsprobleme der Entwicklungsländer, a.a.O., S. $509 \mathrm{ff}$. 
Produkten und Rohstoffen auszudehnen, um so eventuelle Schwankungen im Preis oder Absatzvolumen einer Ware besser ausgleichen zu können. Weiterhin eröffnet sich so die Möglichkeit, durch das Erschließen neuer Märkte zusätzliche Devisenquellen zu erschließen.

Bezüglich der Versorgungssicherheit mit mineralischen Rohstoffen ist das Interesse der Europäischen Gemeinschaft prinzipiell stark ausgeprägt. Zwar besteht gegenwärtig auf den Märkten mineralischer Rohstoffe keine Gefahr eines totalen Belieferungsstopps, jedoch ist die Europäische Gemeinschaft in Anbetracht einer möglichen, kurzfristigen Unterversorgung und der Tatsache, daß sie selbst nur mit geringen Rohstoffvorräten ausgestattet ist, bestrebt, ihre Bezugsquellen zu diversifizieren. Auf diese Weise wird der Versuch unternommen, die Abhängigkeit von wenigen Rohstofflieferanten $\mathrm{zu}$ reduzieren ${ }^{73}$ sowie durch ein ausreichendes Angebot unangemessene Preisentwicklungen zu vermeiden. Da die AKP-Staaten jedoch auf den Weltrohstoffmärkten in der Regel keine dominierende Rolle spielen, ist allein durch das Lome-Abkommen eine Stabilisierung des Preisniveaus nicht zu erreichen.

In der Europäischen Gemeinschaft ist das Bedürfnis der Versorgungssicherheit bei agrarischen Rohstoffen - im Gegensatz zu den mineralischen Rohstoffen - nicht ganz so deutlich ausgeprägt. Die andersgeartete Marktstruktur - es herrscht hauptsächlich eine polypolistische Anbieterseite vor - und die Tatsache, daß es sich bei den in den AKP-Staaten produzierten, agrarischen Rohstoffen häufig um substituierbare (z.B. Kopra, Kautschuk), respektive verzichtbare (z.B. Früchte) Produkte handelt, begründet zwar ein gewisses Interesse an einer gesicherten Belieferung, als besonders intensiv kann es aber nicht eingestuft werden.

Bezüglich Infrastrukturverbesserungen und der Industrialisierung in den AKP-Staaten zeigen verständlicherweise beide Lomé-Vertragsgruppen ein starkes Interesse. An den Infrastrukturmaßnahmen hat die Europäische Gemeinschaft ein reges Interesse, sowohl aus entwicklungs- als auch aus

${ }^{73}$ Zum Beispiel von den westlich orientierten Bergbauländern, besonders von der in den achziger Jahren politisch umstrittenen Republik Südafrika, die einige mineralische Rohstoffe in bedeutenden Mengen nach dergf Europäischen Gemeinschaft liefert: im Jahre 1983 u.a. Chrom 46,8 \%, Zirkon 40,1 \%, Mangan 37,7 \%, Flour 20,9 \%; Vgl. Statistisches Amt der EG (Hrsg.): Rohstoffe, Versorgung der EG 1975 . 1983, Luxemburg 1986, S. 64 - 66. 
wirtschaftspolitischen Gründen. Letzteres erklärt sich wiederum aus einer möglichen Markterschließung (bei Auftragserteilung an europäische Unternehmen) und ist vermutlich stärker ausgeprägt als bei Industrialisierungsprojekten, welche sich unter Umständen einmal zur Konkurrenz gegenüber europäischen Unternehmen entwickeln könnten.

Aufgrund des Lomé-Abkommens werden sich in der Europäischen Gemeinschaft schwerlich neue Industrien etablieren. Anders verhält es sich bei der Arbeitsplatzbeschaffung oder -sicherung. Hier herrscht naturgemäß sowohl bei den AKP-Staaten als auch bei der Europäischen Gemeinschaft äußerstes Interesse. Zu Beginn der achtziger Jahre wurde der Europäischen Gemeinschaft noch von engagierter Seite der Vorwurf gemacht, daß unter dem Deckmantel der Zusammenarbeit wieder nur eigene Belange verfolgt würden. ${ }^{74}$ Hierzu sei angemerkt, daß in internationalen Kooperationsverträgen neben entwicklungspolitischen Aspekten üblicherweise auch wirtschaftliche Belange eine entscheidende Rolle spielen. Dies erscheint auch eine Erklärung für den Austausch der Begriffe Entwicklungshilfe in Entwicklungszusammenarbeit zu sein, wodurch suggeriert werden soll, daß die Entwicklungsländer ebenbürtige Wirtschaftspartner der Industrienationen seien. Dennoch, wie bereits erwähnt, sind seit Lomé III die entwicklungspolitischen Ziele noch stärker in den Vordergrund getreten, die anderen Interessen der Europäischen Gemeinschaft werden nurmehr ansatzweise berücksichtigt.

Technologietransfer ist in den AKP-Staaten sehr erwünscht und muß für den - im Hinblick auf entwicklungspolitische Gesichtspunkte wichtigen weiteren Aus- und Aufbau von Industrien in Entwicklungsländern als grundlegend angesehen werden. Insofern offenbart sich auch in diesem Punkt ein entwicklungspolitisches Interesse der Europäer zusätzlich zu dem wirtschaftspolitischen, welches sich durch die Möglichkeit der Lizenzvergaben und Nachfolgeaufträge erklären läßt - selbst, wenn daraus der europäischen Industrie einmal Mitbewerber erwachsen könnten.

Neben der Schaffung und Ausweitung von Märkten und dem Interesse an der Entwicklungszusammenarbeit läßt sich ein gemeinsames, starkes Interesse an der Reduzierung der Abhängigkeiten von multinationalen Berg-

74 Vgl. Dennhardt, J.; Pater, S. (Hrsg.): Entwicklung muß von unten kommen, Reinbek 1980, S. $40 \mathrm{f}$. 
bau-Unternehmen, sofern sie nicht europäischen Ursprungs sind, feststellen. ${ }^{75}$

Dieser Wunsch, der von seiten der AKP-Staaten durch deren nationalstaatliche Unabhängigkeitsbestrebungen entstanden war, erscheint nur auf den ersten Blick nahe zu liegen.

Es ist richtig, daß ein gewisser Teil der von solchen Unternehmen erwirtschafteten Überschüsse nicht in den Produzentenländern reinvestiert wird, sondern in die Stammhäuser der Konzerne transferiert wird. Diese Tatsache ist für Staaten, die ihre Einnahmen fast ausschließlich aus Rohstoffexporten beziehen, unbefriedigend und führte und führt zu Verstaatlichungsbestrebungen. Auf der anderen Seite aber erweist sich das Einschalten multinationaler Gesellschaften in der Regel als unvermeidbar, wenn überhaupt ein Überschuß erwirtschaftet werden soll. Grund dafür ist ein großes Erfahrungspotential, ein effizientes Management, der Einsatz hochentwickelter Technologien, die Marktkenntnis und die Fähigkeit, das nötige Kapital zu mobiliseren, um so den großen Investitionsbedarf zu decken. ${ }^{76}$

Ähnlich polarisiert sind die Interessen auf europäischer Seite. Zwar besitzt die Bergbauindustrie der Europäischen Gemeinschaft einen hohen Standard, die Möglichkeiten der Einflußnahme auf national-staatliche Entscheidungen sind außerhalb Europas jedoch - von Ausnahmen abgesehen verhältnismäßig gering. ${ }^{77}$ Darüber hinaus ist die Europäische Gemeinschaft in erheblichem Umfange auf die Versorgung durch Bergbaugesellschaften angewiesen, deren Planungs- und Entscheidungszentren außer-

$75 \mathrm{Vgl}$. Kommission der EG (Hrsg.): Bericht der Kommission an den AKP-EWG-Ministerrat über die finanzielle und technische Zusammenarbeit 1982, Kom. (83) 486 endg., Brüssel 29. Juli 1983, S. 8; O.V.: ECC proposes ACP minex programme, in: Mining journal, Vol. 22, 2. Dezember 1983, S. 401; Persönliche Gespräche, Kommission der EG, Brüssel und Bundesministerium für Wirtschaft, Bonn.

${ }^{76} \mathrm{Vgl}$. Kommission der EG (Hrsg.): Bericht der Kommission an den AKP-EWG-Ministerrat ..., a.a.O., S. 8.

$77 \mathrm{Vgl}$. Kommission der EG (Hrsg.): Bericht der Kommission an den AKP-EWG-Ministerrat ..., a.a.O., S. 8. 
halb Europas liegen. ${ }^{78}$ Daher ist prinzipiell die Gefahr einer Unterversorgung in eventuellen Krisenzeiten nicht auszuschließen. Ein Versorgungsengpaß kann besonders dann sehr schnell akut werden, wenn von europäischer Seite keine ausreichende Kontrolle auf die Bezugsquellen ausgeübt werden kann. Diese Tatsache wird durch die seit den siebziger Jahren feststellbaren Veränderungen in den Investitions- und Produktionsbedingungen zugunsten nationaler Interessen - auch in den westlich orientierten Bergbauländern - verstärkt. ${ }^{79}$

Bisher jedoch sind die multinationalen Unternehmen der westlich orientierten Bergbauländer - selbst in Engpaßsituationen ${ }^{80}$ - generell ihren Lieferverpflichtungen nachgekommen. ${ }^{81}$ Vielmehr sind sie wohl am ehesten in der Lage, durch ihre internationalen Beziehungen regionale Versorgungsschwierigkeiten - zum Beispiel auch durch Streiks - zu kompensieren. Dennoch ist das starke Interesse der Gemeinschaft an einer Diversifizierung des Rohstoffbezuges und einer Reduzierung der Abhängigkeit von wenigen außereuropäischen Bergbauunternehmen berechtigt.

Eng verbunden mit den Diversifikationsbestrebungen ist das Interesse europäischer Unternehmen am Schutz ihrer Investitionen in den AKP-Staaten. Daß auf diesem Gebiet noch große Unsicherheit herrscht, zeigen die geringen Investitionen europäischer Bergbaugesellschaften in den Entwicklungsländern. Für Anfang der achtziger Jahre werden folgende Prozentsätze angegeben:

Afrika 2 Prozent, Lateinamerika 15 Prozent, Asien und Ozeanien 1 Prozent, OECD-Länder 82 Prozent $^{82}$ (s. Tab. 5).

${ }^{78}$ Dies trifft sowohl in den Bergbaunationen USA, Kanada, Australien und Republik Südafrika als auch in Entwicklungsländern zu, wo nationale Bergbaugesellschaften immer mehr an Bedeutung gewonnen haben.

${ }^{79} \mathrm{Vgl}$. Harms, U., et al.: Die Investitionspolitik der NE-Bergbaugesellschaften, ite, Hamburg 1978, S. 1.

${ }^{80}$ Es sei an die Ölkrise $1973 / 74$ erinnert, wo beispielsweise Exxon auch weiterhin Öl nach Europa lieferte.

${ }^{81}$ Vgl. Harms, U. et al.: Die Investitionspolitik ..., a.a.O., S. 1

${ }^{82}$ Kommission der EG (Hrsg.): Bericht der Kommission an den EG-Ministerrat ..., a.a.O., S. 13; Vgl. auch Harms, U. et al.: Die Investitionspolitik ..., a.a.O., S. 6. 
Zusammenfassend läßt sich feststellen, daß die AKP-Staaten ein vehementes Interesse an den Lome-Abkommen haben und auch in Zukunft an einer Fortführung dieser Vereinbarungen sehr interessiert sein werden. Wesentlich differenzierter sieht die Interessenslage bei der Europäischen Gemeinschaft aus. Anhand der tabellarischen Auflistung (Abb. 2) ist jedoch ersichtlich, daß auch hier ein über entwicklungspolitische Aspekte hinausgehendes Interesse besteht, diese Art von Abkommen künftig weiterzuführen, auch wenn Positionen, die für die europäischen Belange von Interesse sind, im Text der folgenden Verträge wahrscheinlich immer weniger Berücksichtigung finden.

\subsection{Die Organe der Lomé-Abkommen}

In den Lome-Abkommen sind drei Organe vorgesehen:

- der Ministerrat,

- der Botschafterausschuß,

- die Paritätische Versammlung (bis Lomé II: Beratende Versammlung AKP-EWG).

Diese Organe bestehen seit dem ersten Abkommen, ihre Funktionsfähigkeit wurde besonders in den Lome-III-Abkommen optimiert. In Lomé III wird erstmals getrennt von den Aufgaben der Organe (Teil I) die Arbeitsweise der einzelnen Organe (Teil 4) festgelegt.

Der Ministerrat ist die oberste beschlußfähige Instanz und tritt mindestens einmal jährlich zusammen. Er besteht aus den Mitgliedern des Rates der Europäischen Gemeinschaften, Mitgliedern der EG-Kommission, je einem Mitglied der AKP-Staaten und unter Umständen einem Vertreter der EIB. Der Ministerrat publiziert einen jährlichen Tätigkeitsbericht, für wichtige Aufgaben kann er Ausschüsse einsetzen. In Lomé IV erhält der Ministerrat weitere Kompetenzen zur Überprüfung seiner Initiativen (Lomé IV: Art. 343).

Der Botschafterausschuß besteht aus Vertretern der EG-Staaten, der AKPLänder und der EG-Kommission sowie unter Umständen einem Vertreter der EIB. Der Ausschuß unterstützt den Ministerrat und übernimmt vom 
Ministerrat übertragende Aufgaben. Des weiteren überwacht er die Arbeiten aller Gremien, Ausschüsse oder Arbeitsgruppen.

Die Paritätische Versammlung besteht zu gleichen Teilen aus den Mitgliedern des Europa-Parlamentes und aus Parlamentsmitgliedern der AKPStaaten. Sie tritt zweimal jährlich zusammen. Dabei erhält sie den Tätigkeitsbericht des Ministerrates, verabschiedet Entschließungen, die das Abkommen betreffen und legt Empfehlungen vor, um die EG-AKP-Zusammenarbeit $\mathrm{zu}$ intensivieren.

Der Ministerrat - und in seiner Vertretung der Botschafterausschuß - äuBert sich nur im gegenseitigen Einvernehmen der Gemeinschaft einerseits und det AKP-Staaten andererseits (Lomé IV: Art. 338). Durch diese Regelung existiert zum einen die Möglichkeit, daß beide Verhandlungsgruppen ihre unterschiedlichen Auffassungen dokumentieren können. Zum anderen wird jedoch verhindert, daß Differenzen innerhalb der einzelnen Vertragspartner nach außen getragen werden.

Im Falle von Streitigkeiten fällt zunãchst dem Botschafterausschuß, dann dem Ministerrat die Vermittlerrolle zu. Sind die Unstimmigkeiten nicht beizulegen, wird vom Ministerrat ein Schiedsverfahren eingeleitet. Beide Kontrahenten ernennen je einen Schiedsrichter, diese - oder der Kopräsident des Ministerrates - wiederum einen dritten.

Dem Schiedsspruch der drei Richter ist von den beteiligten Parteien folge zu leisten (Lomé IV: Art. 352).

\subsection{Die Instrumente der Lomé-Abkommen}

Während das Lomé-II-Abkommen lediglich eine Weiterentwicklung der Vorgängerversion darstellte, handelte es sich bei Lomé III - wie bereits am Aufbau zu erkennen - um eine Neukonzeption. Das Lomé-IV-Abkommen ist auf der Grundlage von Lome III weiterentwickelt worden. Dabei wurden jedoch zum Teil wesentliche Modifikationen vorgenommen beziehungsweise es sind zusätzlich neue Instrumente vereinbart worden. Besonders zu erwähnen ist hier die Verlängerung der Laufzeit von fünf auf zehn Jahre, wobei die Mittel der Europäischen Gemeinschaft (EEF sowie 
EIB-Mittel) nach wie vor im Fünf-Jahres-Turnus bereitgestellt und dann neu verhandelt werden. ${ }^{83}$

In den beiden ersten Abkommen sind nur die Instrumente der AKP-EGKooperation definiert worden, seit Lomé III dagegen wird zunächst der Definition der Zusammenarbeit und der Festlegung der Kooperationsgebiete und -ziele breiter Raum eingeräumt. Erst im dritten Teil werden die einzelnen Instrumente der Zusammenarbeit aufgeführt.

In Lomé IV wurden zu den im zweiten Teil des Lomé-III-Abkommens festgehaltenen, sogenannten Bereichen der Zusammenarbeit, wie Entwicklung der ländlichen Regionen, Entwicklung von Landwirtschaft, Industrie und Handel sowie des Bergbau- und Energiepotentiales neu hinzugenommenen unter anderem Umwelt, Entwicklung von Handwerk und Weiterverarbeitung sowie Dienstleistungen einschließlich Tourismus. ${ }^{84}$

Die im dritten Teil des Lomé-IV-Abkommens definierten Instrumente der AKP-EG-Kooperation unterscheiden sich prinzipiell nicht von denjenigen der Vorgängerkonventionen. Jedoch wurden die einzelnen Maßnahmen zum Teil radikal überarbeitet und häufig erweitert, um so deren Anwendung zweckmäßiger und wirkungsvoller zu gestalten. In Lomé IV neu vereinbart wurde unter anderem die Unterstützung bei der Schulden- und Strukturanpassung. ${ }^{85}$

\subsubsection{Die handelspolitischen Instrumente}

Aufgrund der handelspolitischen Regelungen können die AKP-Staaten den weitaus größten Teil ihrer Erzeugnisse zollfrei in die Gemeinschaft exportieren, ohne den europäischen Produkten ihrerseits Zollfreiheit einräumen zu müssen.

Diese Vereinbarungen sind bei den jeweiligen Neuverhandlungen weiter zugunsten der AKP-Staaten verbessert worden, die zollfreie Einfuhr nach

${ }^{83} \mathrm{Vgl}$. Finanzprotokoll im Anhang zum Lomé-IV-Abkommen, Artikel 1.

${ }^{84} \mathrm{Vgl}$. O.V.: Details on principal developments and innovations, in: The Courier, Nr. 120, März - April 1990, S. 14.

${ }^{85}$ Ebenda, S. 14 f. 
der Europäischen Gemeinschaft wurde bis auf wenige Agrarerzeugnisse auf sämtliche AKP-Ursprungswaren ausgedehnt. Das Präferenzsystem für diese Produkte, sie fallen unter die EG-Marktordnung, ist auch bei den Verhandlungen zu Lomé IV weiter optimiert worden. ${ }^{86}$

Diese Vereinbarungen galten bei ihrer Einführung als große, politisch fundamentale Neuerung ${ }^{87}$ zumal die Idee der Freihandelszone mit beiderseitigem Abbau der Handelshemmnisse schon bei den Verhandlungen zum Lome-I-Abkommen auf Druck der AKP-Staaten fallen gelassen wurde. Daher existiert auch in Lomé IV nur eine Meistbegünstigten-Klausel, worin sich die AKP-Staaten verpflichten, die Europäische Gemeinschaft nicht schlechter zu stellen als Drittländer (Lomé IV: Art. 174).

In der ersten Hälfte der achtziger Jahre wurden zwischen 32 Prozent im Jahre 1981 und 42 Prozent im Jahre 1983 der AKP-Exporte (Warenwert) nach der Europäischen Gemeinschaft geliefert; die AKP-Staaten spielen für den Handel der Europäischen Gemeinschaft dagegen nach wie vor nur eine untergeordnete Rolle. (s. Abb. 3).

Trotz der weitgehenden Zugeständnisse in den Lomé-Abkommen gegenüber den AKP-Staaten hat sich die Struktur des Warentausches mit der Europäischen Gemeinschaft in den achtziger Jahren nicht wesentlich verändert, sie ist immer noch unausgewogen ${ }^{88}$ (s. Abb. 4).

${ }^{86} \mathrm{Vgl}$. O.V.: Das neue Lomé-Abkommen (II), in: Europa-Archiv, Dokumente, Folge 21, 1979, S. D 578; Bundesstelle für Außenhandelsinformation (Hrsg.): EWG und AKP-Staaten, Lome-III-Abkommen, in: Markt-Information, Reihe Ml-C, Köln, Februar 1985, S. 3; O.V.: Details on principal development and innovations, a.a.O., S. $15 \mathrm{f}$.

${ }^{87} \mathrm{Vgl}$. Krohn, H.B.: Das Abkommen von Lomé (I) ..., a.a.O., S. 181.

${ }^{88} \mathrm{Vgl}$. O.V.: EEC-ACP trade, statistical analysis 1970 - 1981, in: The Courier, Nr. 79, Mai - Juni 1983, S. 59; Statistisches Amt der EG (Hrsg.): Foreign trade: third countries ..., a.a.O., S. 28; Institut für Weltwirtschaft (Hrsg.): ACP-EEC trade (1984), in: The Courier, Nr. 98, Juli - August 1986, S. 64 f; BMZ (Hrsg.): Lomé IV, in: Entwicklungspolitik, Materialien 82, Bonn (1991), S. 131. 
Abbildung 3: Der Handel zwischen EG, AKP-Staaten und übriger Welt 1981 und 1991 (in Mrd. ECU; nominal)
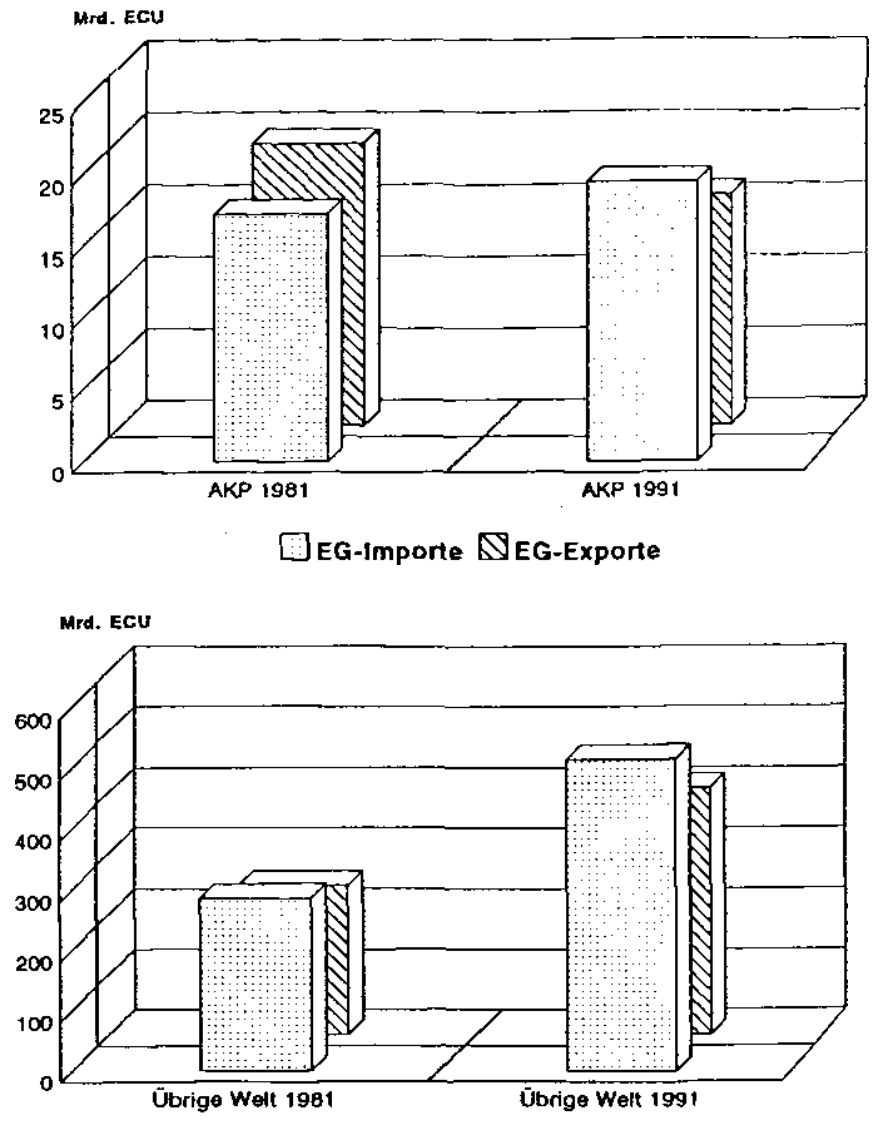

BEG-Importe QEG-Exporte

Quelle: Eigene Zusammenstellung nach: Statistisches Amt der EG (Hrsg.): Key figures, Supplement - Target 92, No 6-1992, S.3. 
Somit hat die Kritik, die zu Beginn der Laufzeit von Lomé II geäußert wurde, daß die Öffnung des EG-Marktes zugunsten der AKP-Staaten als eine eher "eindrucksvolle" als "wirkungsvolle Geste" zu bezeichnen sei, ${ }^{89}$ durchaus ihre Rechtfertigung. Dieser Tatsache wurde bei den Verhandlungen zum Vierten Abkommen von Lomé insofern Rechnung getragen, als ein neuer Titel Handelsentwicklung aufgenommen wurde. Dadurch soll gewährleistet werden, daß die AKP-Staaten einen optimalen Nutzen aus den handelspolitischen Vereinbarungen des Abkommens erzielen können (Lomé IV: Art. 135 ff.).

Abbildung 4: Der EG-AKP-Handel 1988, gesamt sowie einige SITC-Warenklassen (in Mrd. ECU)

Mrd. ECU

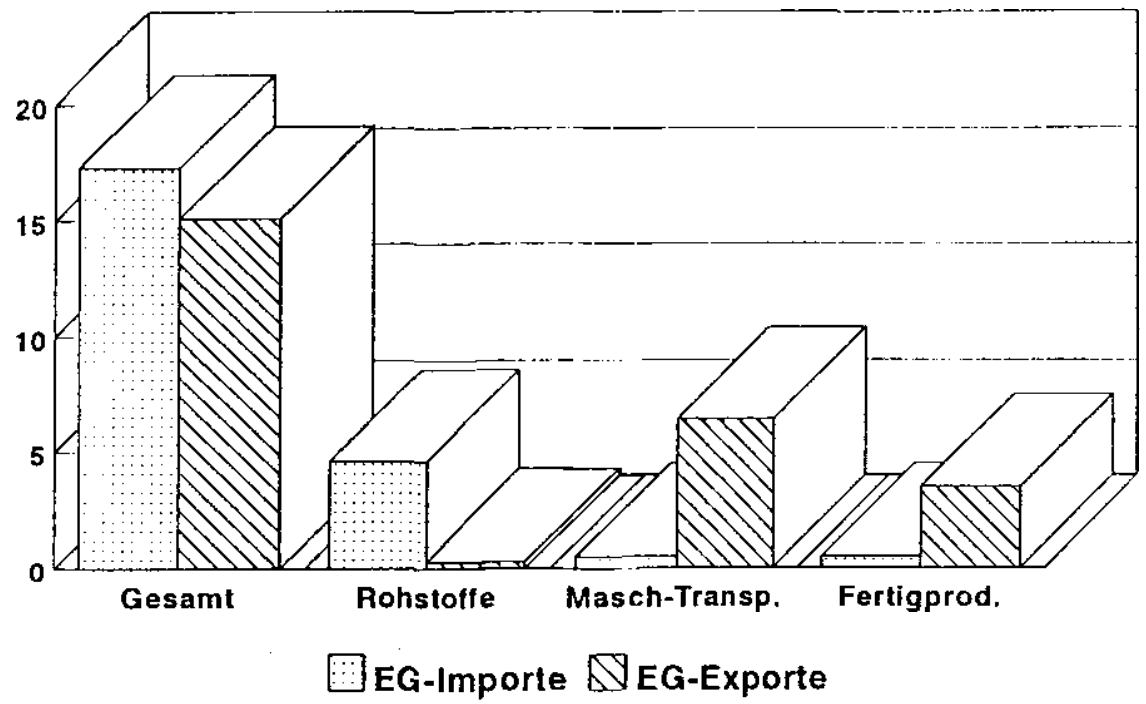

Quelle: Eigene Zusammenstellung nach: Eurostat, in: Bundesministerium für Wirtschaftliche Zusammenarbeit (Hrsg.): Lomé IV, Entwicklungspolitik, Materialien Nr. 82, Bonn (1991), S. 131.

${ }^{89} \mathrm{Vgl}$. Focke, K.: Von Lomé 1 zu Lomé 2, a.a.O., S. 14. 
2.5.2 Stabilisierung der Erlöse aus der Ausfuhr von landwirtschaftlichen Grundstoffen (Stahex)

Für Entwicklungsländer sind die Erlöse aus dem Export von Roh- und Grundstoffen nach wie vor die wichtigste Quelle für ihre Deviseneinnahmen. Negative Einflüsse durch Schwankungen der Ausfuhrerlöse auf die Zahlungsbilanz eines Entwicklungslandes sind erheblich, besonders wenn die Exporte, wie in den meisten AKP-Staaten, im wesentlichen nur aus einem oder wenigen Rohstoffen bestehen.

Neben einer ungünstigen Wirkung auf das Haushaltsgleichgewicht - öffentliche Ausgaben können bei sinkenden Einnahmen nicht schnell genug gedrosselt werden - verhindern reduzierte Deviseneinnahmen, daß unter Umständen notwendige Einfuhren getätigt werden können; beispielsweise sah sich Sambias Bergbauindustrie in der ersten Hälfte der achtziger Jahre nicht in der Lage, ausreichende Mengen von Dieselkraftstoff und dringend benötigte Ersatzteile zu beschaffen, da Devisen fehlten. ${ }^{90}$ In extremen Fällen können die Exporterlösschwankungen dazu beitragen, daß die internationale Kreditwürdigkeit eines betroffenen Entwicklungslandes zusätzlich beeinträchtigt wird ${ }^{91}$ Somit sind Aktionen zur Stabilisierung der Devisenerlöse als notwendige Vorbereitung für eine gesteigerte Selbsthilfe der Entwicklungsländer anzusehen. ${ }^{92}$

Entsprechend dieser Erkenntnis ist bereits im Lomé-I-Abkommen ein System zur Stabilisierung der Exporterlöse (Stabex) eingeführt worden.

Das Konzept des Stabex-Verfahrens basiert auf einem produktbezogenen Erlösausgleich. Sobald ein AKP-Staat bei seinen Exporten nach der Europäischen Gemeinschaft innerhalb einer Referenzperiode von 6 Jahren $^{93}$

${ }^{90}$ Persönliche Gespräche, ZCCM, Sambia 1984.

${ }^{91}$ Vgl. Kommission der EG (Hrsg.): Zusammenfassender Bericht über das mit dem Abkommen von Lomé eingeführte System zur Stabilisierung der Ausfuhrerlöse in den Anwendungsjahren 1975 bis 1979, Sek (81) 1104, Brüssel 1981, S. 3 und S. 68.

92 Vgl. Kröner, E.: Das Stabex-System, zum Ausgleich der Exporterlösschwankungen, in: Dams, T.; Grohs, G.: Kontroversen in der internationalen Rohstoffpolitik, München 1977, S. 145; Hemmer, H.R.: Wirtschaftsprobleme der Entwicklungsländer, a.a.O., S. 748 f.

${ }^{93}$ Abzüglich der zwei Jahre mit den höchsten bzw. niedrigsten Ergebnissen (Lomé IV: Art. 197 Abs. 2). 
Erlöseinbußen erleidet, die eine Auslöseschwelle von 4,5 Prozent (Lome I: 7,5 Prozent) erreichen oder überschreiten, entsteht automatisch ein Anspruch auf Ausgleichszahlungen aus dem Stabex-Fonds. ${ }^{94}$ Die Voraussetzung für einen Anspruch auf Stabex-Transfers ist allerdings die Exportabhängigkeit des AKP-Staates von den betroffenen Produkten in Höhe von mindestens 5 Prozent (Lomé I: 7,5 Prozent); für Sisal beträgt die Abhängigkeitsschwelle nur 4 Prozent.

Bei den am wenigsten entwickelten Länder (LDC), Binnen- und Inselstaaten reduzieren sich der Schwellenwert auf 1 Prozent (Lomé I: 2,5 Prozent).

Die zunächst 12 Warengruppen und 29 Produkte, die von der "Versicherung gegen schlechte Jahre" erfaßt wurden, ${ }^{95}$ sind in Lomé IV auf 32 Gruppen und 49 Produkte erweitert worden. Bei einer Warengruppe handelt es sich beispielsweise um Kakao, der sich aus den Produkten Kakaobohnen, -schalen, -paste, -butter oder -puder zusammensetzt. Stabex-Leistungen werden sowohl für ein einzelnes Produkt als auch für eine Warengruppe gewährt. Auf Antrag können weitere Produkte in den Stabex-Katalog aufgenommen werden.

Die Berücksichtigung der Abhängigkeit einiger AKP-Staaten vom Export mineralischer Rohstoffe war im Ersten Abkommen von Lomé noch wenig ausgeprägt. Lediglich die Ausfuhren von Eisenerzen und -konzentraten und Schwefelkiesabbränden waren auf Antrag Liberias im Stabex-Verfahren von Lomé I erfaßt worden. Dieser Aufnahme stimmte die EG-Verhandlungsdelegation nur zu, "um die gesamte Regelung nicht in Frage zu stellen". .96

Vier afrikanische Staaten machten von der Möglichkeit Gebrauch, Transferleistungen aufgrund von Erlösrückgängen bei Eisenerzen zu beantragen. In den Jahren 1976 bis 1979 erhielten die Länder Swasiland, Sierra

${ }^{94}$ Seit Lome IV werden nunmehr auch Exporte nach anderen AKP-Staaten oder unter gewissen Voraussetzungen auch nach sonstigen Bestimmungen berücksichtigt; in Lomé III war dies nur auf Antrag möglich (Lomé IV: Art. 179; Lomé III: Art. 150).

${ }^{95}$ Focke, K.: Von Lomé 1 zu Lomé 2, a.a.O., S. 22.

$96 \mathrm{Vgl}$. Kommission der EG (Hrsg.): Zusammenfassender Bericht ..., a.a.O., S. 10. 
Leone, Mauretanien und Liberia Ausgleichszahlungen in Höhe von rund 62 Mio. $\operatorname{ECU}^{97}$ (s. Tab. 2).

Der Rückgang der Exporterlöse resultierte sowohl aus konjunkturellen als auch aus regionalen Ereignissen. Die Ansprüche von Mauretanien und $\mathrm{Li}$ beria auf Stabex-Transfers wurden durch konjunkturelle Ursachen ausge-löst. Der Weltmarktpreis für Eisenerze nahm seit dem Jahre 1975 ab und erreichte im Jahre 1978 einen Tiefpunkt. Selbst im Jahre 1980 war der inflationsbereinigte Jahrespreis noch nicht wieder auf dem Stand des Jahres $1977^{98}$ (s. Abb. 5).

Das Stabex-Verfahren erscheint seinen Zweck erfüllt zu haben: Die Exporterlöse für Eisenerze sanken, und die Stabex-Transfers stiegen proportional dazu an. Die in Höhe und Auszahlungszeitraum unterschiedlichen Stabex-Darlehen dokumentieren die individuelle Handhabung dieses Verfahrens (s. Tab. 2 und Abb. 5).

Obwohl in Mauretanien und Liberia aufgrund der wieder angestiegenen Exporterlöse keine weiteren Ansprüche auf Darlehen aus dem StabexFonds erwachsen waren, konnte weder in der Produktion noch im Exportvolumen nach der Europäischen Gemeinschaft eine wesentliche Trendumkehr im Vergleich zu den Vorjahren verzeichnet werden (s. Tab. 3 und Abb. 5). Aussagen, inwieweit die Mittel der Stabex-Darlehen in die betroffenen Bergwerke geflossen sind oder für andere Vorhaben der jeweiligen Regierung verwendet wurden, lassen sich allein aufgrund der Produktions- und Exportdaten nicht treffen.

Spekulationen, ob die Bergbauunternehmen vor der Antragstellung auf Stabex-Darlehen ihren Exportausstoß nochmals kurzfristig gesteigert hatten, so daß der darauffolgende Rückgang drastischer ausgefallen ist, und somit eventuell ein größerer Transferbetrag zugestanden werden mußte, ${ }^{99}$ erscheinen in diesen Fällen eher unwahrscheinlich zu sein. Während bei agrarischen Erzeugnissen solche Manipulationen durchaus schon nachge-

$97 \mathrm{Vgl}$. Kommission der EG (Hrsg.): Zusammenfassender Bericht ..., a.a.O., S. 33.

98 Vgl. Saager, R.: Metallische Rohstoffe von Antimon bis Zirkonium, Zürich 1984, S. 106.

99 Persönliches Gespräch, Bundesministerium für Wirtschaft, Bonn; Kommission der EG, Brüssel. 
wiesen werden konnten, ${ }^{100}$ ist ein solches Verhalten bei mineralischen Rohstoffen weniger zu erwarten. Zum einen sind Preisentwicklungen bei mineralischen Rohstoffen nur selten mit hinreichender Genauigkeit zu antizipieren, zum anderen bestehen bei Bergbauerzeugnissen in der Regel längerfristige Lieferkontrakte. ${ }^{101}$ Darüber hinaus ist das Risiko, daß die Manipulation entdeckt wird, gegenüber der geringen Transferhöhe unverhältnismäßig hoch.

Die Stabex-Transfers an Sierra Leone und Swaziland bestätigen die Aussage, daß Stabex-Darlehen nicht zur Sicherung der EG-Versorgung beitragen. In beiden Fällen sind die Ansprüche - außer von Swasiland im Jahre 1977 - aus regionalen Schwierigkeiten erwachsen und wurden zur Schließung der Bergwerke verwendet. ${ }^{102}$

Damit besaßen diese Maßnahmen nahezu ausschließlich sozialen Charakter (Weiterbeschäftigungsmöglichkeiten, Umschulungsprogramme), welches den Grundsätzen des Stabex-Verfahrens entsprach und entspricht: "... den wirtschaftlichen und sozialen Fortschritt ihrer Bevölkerung zu sichern und zum Schutz ihrer Kaufkraft beizutragen" (Lomé IV: Art. 186, Abs. 1; Lomé II: Art. 23, Abs. 1). Mit rohstoffpolitischen Konzepten der Europäischen Gemeinschaft können solche Aktionen jedoch nicht in Einklang gebracht werden.

Verdeutlicht wird dieser Umstand darüber hinaus durch die Tatsache, daß Sierra Leone abbauwürdige Eisenerzvorkommen besitzt, in welchen ab Mitte 1982 die Produktion wieder aufgenommen wurde; seit Februar 1983 wird wieder exportiert. ${ }^{103}$

${ }^{100}$ In 1988 hatte die Elfenbeinküste in Erwartung höherer Preise einen Großteil ihrer Kakaoexporte zurückgestellt. Nach Ausbleiben der Preiserhöhung wurden Stabex-Ansprüche geltend gemacht; vgl. O.V.: Stabex: Teillösung für das Defizit im Anwendungsjahr 1988, in: Europa Entwicklung, Oktober 1989, Nr.7, Brüssel, S. 4.

$101 \mathrm{Vgl}$. Wagner, W. et al.: Die wirtschaftliche und rohstoffpolitische Bedeutung Afrikas, ite, Hamburg, Januar 1981, S. 245.

$102 \mathrm{Vgl}$. Kommission der EG (Hrsg.): Zusammenfassender Bericht der Kommission an den AKP-EWG-Ministerrat ..., a.a.O., Tabellen I, 11 und I, 13.

${ }^{103}$ Vgl. Glaser, T.: Sierra Leone, in: The Courier, Nr. 86, Juli - August 1984, S. 25. 
Tabelle 2: Stabex-Transfers für Eisenerze während des Lomé-I-Abkommens (in Mio. ECU)

\begin{tabular}{|c|c|c|c|c|c|}
\hline & 1976 & 1977 & 1978 & 1979 & Total \\
\hline Slerra Leone & 3,977 & - & - & - & \\
\hline Swasiland & - & 3,369 & 5,490 & 4,366 & \\
\hline Mauretanien & - & 3,606 & 33,395 & - & 61,788 \\
\hline Liberta & - & - & 7,587 & - & \\
\hline $\begin{array}{l}\text { der Stabex- } \\
\text { Transfers }\end{array}$ & 10,71 & $20 ; 68$ & 28,34 & 6,98 & 16,7 \\
\hline
\end{tabular}

Anm.: Angabe gerundet

Quelle: Kommission der EG (Hrsg.): Zusammenfassender Berlcht über das mit dem Abkommen von Lomé elngeführte System zur Stabilisierung der Ausfuhrerlöse in den Anwendungsjahren 1975 bis 1979, Brüssel 1981, Tabellen I, 1 und $I, 8$. 
Tabelle 3: Elaenerz-produktion und-Export nach der EG dex vier Akp-Staaten, die für den Bergbausektor stabex-Darlehen erhalten haben

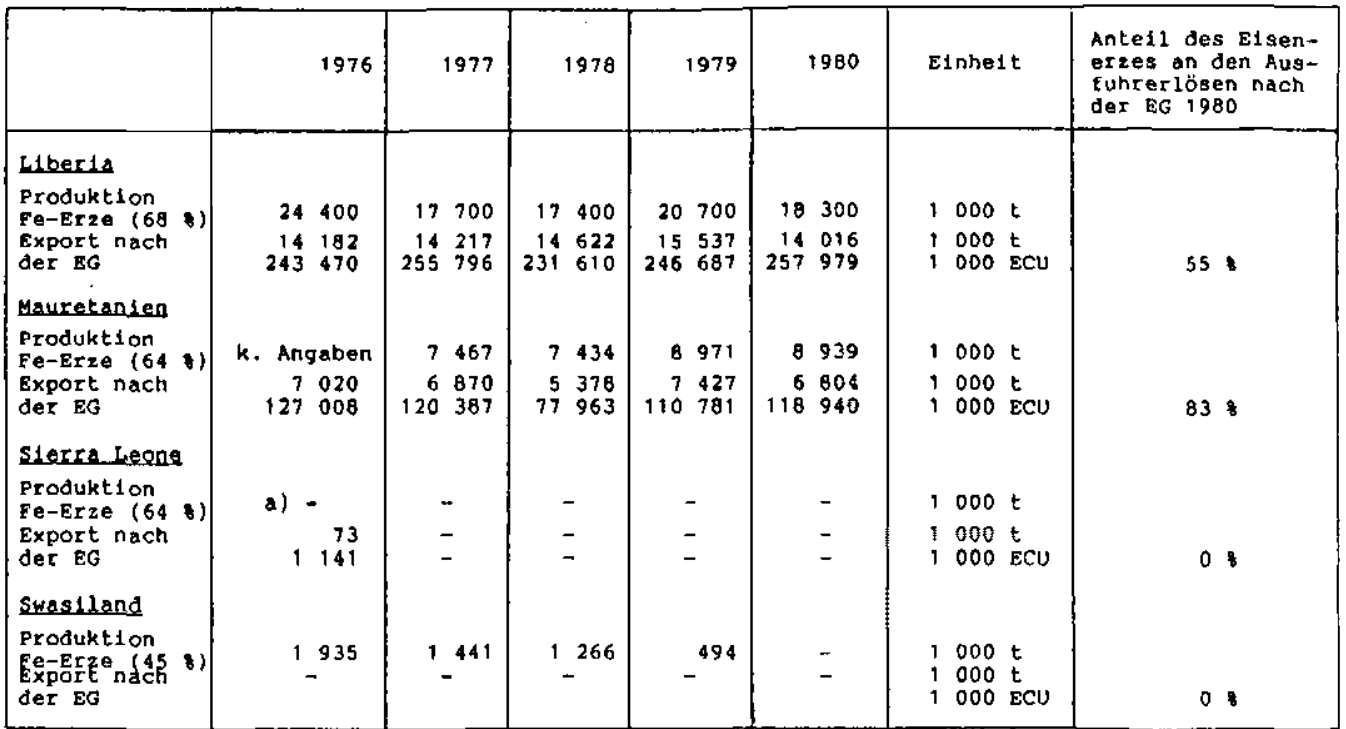

Anm.: Die fn Klammern gesetzten Prozentzahlen beziehen alch auf den durchachnittlichen E1sena) Dle Produktion wurde Ende 1975 eingestellt; vgl. Mining Annual Review 1979, S. 497.

Quelien: Kommission der EG (Hrsg.): Importations CEE des principaux prodults, 0.0. ,

3. Dezember 1981, s. $40-71$; Statistisches Bundesamt wiesbaden (Hrsg.):

Länderkurzberichte Liberia; Mauretanien; Sierza Leone; Swasiland,

wesbaden 1982 - 19B3 
Abbildung 5: Eisenerz-Exporte Liberias und Mauretaniens nach der EG sowie Stabex-Darlehen, die durch Exporterlösrückgänge der Eisenerze ausgelöst wurden

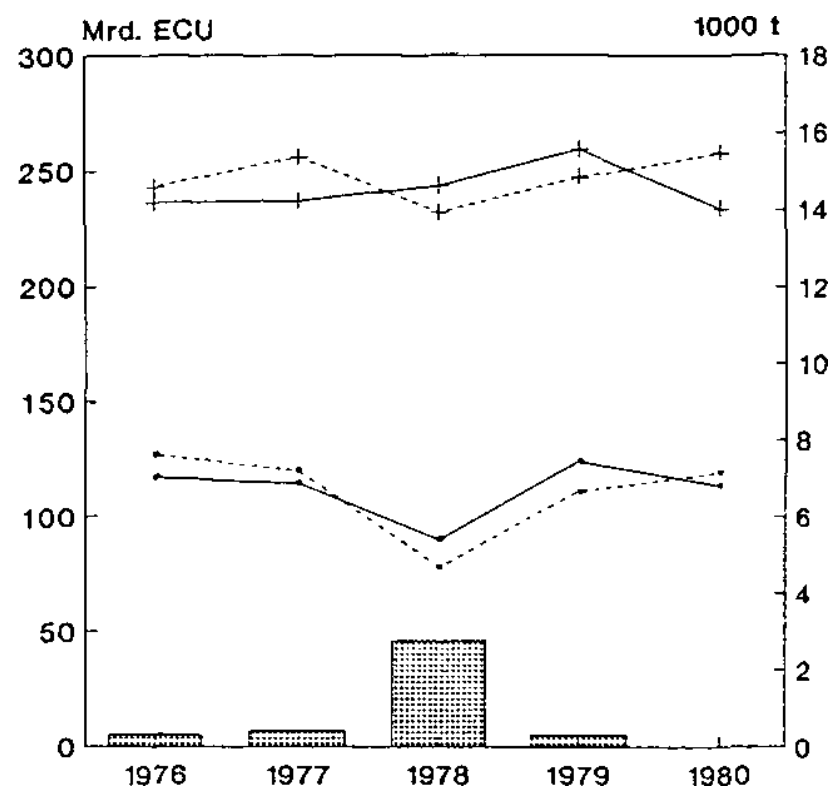

Anm :

-t Menge Liberia - Menge Mauretanien

+. Exporterlöse Liberia … Exporterlöse Mauretanien Stabex-Darlehen ausgelöst durch Eisenerz Exporterlös-Rückgänge

Quelle: Eigene Zusammenstellung, Angaben nach Tabelle 2 und 3. 
Ein Transfer nach dem Sysmin-Verfahren hätte in diesem Falle weitergehende Maßnahmen ausgelöst - nicht Stillegung, sondern Aufrechterhaltung der Produktionskapazitäten - und wäre so deutlicher den Interessen einer europäischen Rohstoffversorgungspolitik entgegengekommen.

Bei den Neuverhandlungen zu Lomé II sprach sich die Europäische Gemeinschaft gegen eine weitere Aufnahme von Bergbauerzeugnissen in das Stabex-System aus. Als ausschlaggebendes Argument wurde der enge Finanzrahmen angegeben. ${ }^{104}$

Eine Übergangsregelung in Lomé II bezog Eisenerze und Schwefelkiesabbrände noch in das Stabex-Verfahren ein, falls sie in Bergwerken gefördert wurden, die bereits vor der Lomé-II-Unterzeichnung erschlossen waren. Zu einer Anwendung dieser Regelung ist es allerdings nicht gekommen.

Während des Lomé-I-Abkommens wurden 123 Transferbeschlüsse zugunsten von 37 der damals 52 AKP-Länder und 2 überseeischen Gebieten (ÜLG) durchgeführt. Von den 32 Waren, die in Lomé I erfaßt wurden, sind 24 durch das Stabex-System gestützt worden. Zwei Drittel der Transferzahlungen allerdings wurden Jediglich durch 3 Produkte bzw. Produktgruppen in Anspruch genommen: Erdnußwaren, Eisenerze und Baumwolle. Unter Lomé II wurden Stabex-Leistungen in Höhe von 659,5 Mio. ECU - gegenüber einem ursprünglichen Budget von 557 Mio. ECU - an 46 AKP-Staaten und ÜLG ausgezahlt. Die Transfers waren zu 75 Prozent auf nur 3 Rohstoffe: Kaffee, Kakao und Erdnüsse konzentriert. Die größten Nutznießer der Stabex-Regelung während der Laufzeit des Lomé-Il-Abkommens sind die Länder Senegal (13,7 Prozent), Ghana (12,9 Prozent) und Elfenbeinküste (11,9 Prozent) gewesen. ${ }^{105}$ Unter dem Stabex-Verfahren von Lomé III wurden bis zum 31. Dezember 1988 Transfers in Höhe von 919 Mio. ECU genehmigt; davon wurden nahezu 60 Prozent der Mittel für Kaffee ausgezahlt, die 3 AKP-Staaten mit den

104 Fralon, J. et al:: The new EEC-ACP-convention: from Lomé I to Lomé II, Brüssel 1980, S. 166, zit. in: Rajana, C.: Europe undermined, the Lome response, in: Africa Development, Vol. VI, Nr. 2, April - Juni 1982, S. 10.

${ }^{105} \mathrm{Vgl}$. Kommission der EG (Hrsg.): Ten years of Lome, Europe Information Development, September 1986, S. 24 f. 
größten Transfers waren die Elfenbeinküste (29,7 Prozent), Äthiopien (15,2 Prozent) und Kamerun (11,3 Prozent). ${ }^{106}$

Bei den durch das Stabex-Verfahren begünstigten AKP-Staaten waren im Vergleich zu einem - seit Lomé III vierjährigen - Referenzniveau Einnahmeminderungen eingetreten, die aus einem Erlösrückgang der jeweiligen Produkte bei der Ausfuhr nach der Europäischen Gemeinschaft resultierten.

Da es sich bei dem Stabex-System um ein Verfahren der Erlös- und nicht der Preisstabilisierung handelt, werden auch die Einnahmeverluste berücksichtigt, die sich aus einer Mengenreduzierung ergeben haben.

Diese Abnahme der Exportmengen kann einmal aus lokalen Ereignissen resultieren, wobei - neben politischen, sozialen und technischen Ursachen - besonders bei agrarischen Erzeugnissen Natürkatastrophen wie Dürre, Wirbelstürme, Kälteeinbrüche oder Pflanzenkrankheiten eine Rolle spielen können. Weiterhin können Nachfrageänderungen in Europa die Exporterlöse eines AKP-Staates mindern, sei es durch einen konjunkturell bedingten Nachfragerückgang oder durch das Auftreten weiterer Anbieter.

Etwa 69 Prozent der Stabex-Transfers während des Lome-I-Abkommens resultierten aus lokalen Ereignissen, in der Regel aus Naturkatastrophen, wie z.B. Dürre, Schädlingsbefall. Etwa 31 Prozent wurden durch konjunkturbedingte Verluste, d.h. Nachfrageänderungen in Europa bedingt. ${ }^{107}$

Die finanzielle Ausstattung des Stabex-Fonds in Lomé I (382 Mio. ECU) reichte aus, um sämtliche Transferanträge zu befriedigen. ${ }^{108}$ Die Vermutung, daß eine Aufstockung der Stabex-Mittel um nahezu 50 Prozent in Lomé II nicht ausreichen würde - bei einer Absenkung der Abhängigkeits-

106 Eigene Berechnung nach: O.V.: Stabex, in: The Courier, Nr. 100, November - Dezember 1986, S. III; Derselbe, Nr. 105, September Oktober 1987, S. IX; Derselbe, Nr. 112, November - Dezember 1988, S. XV; Kommission der EG (Hrsg.): Lomé III - Mid term review 1986 - 1988, Brüssel 1989, S. 16 f; BMZ (Hrsg.): Lomé IV, in: Entwicklungspolitik, Materialien 82, a.a.O., S. 132.

${ }_{107}$ Vgl. Kommission der EG (Hrsg.): Zusammenfassender Bericht ..., a.a.O., S. 33 und Tabelle I, 8.

108 Vgl. Wawrzik, K.: Bericht ..., a.a.O., S. 50. 
und Auslöseschwellen und einer Erweiterung der erfaßten Produkte - bewahrheitete sich bereits im Jahre 1980. Entgegen ursprünglichen Vermutungen der EG-Kommission verschärfte sich die Situation im darauffolgenden Jahr, besonders wegen der hohen Erlösausfälle bei Kaffee und Erdnüssen. Die Differenz zwischen berechtigten Anträgen und verfügbaren Mitteln hatte sich im Jahre 1981 gegenüber dem Vorjahr fast verdreifacht: Ansprüchen in Höhe von 453 Mio. ECU standen nur 112 Mio. ECU der Stabex-Jahrestranche gegenüber. ${ }^{109}$

Durch einen einmaligen Zuschuß, vereinbart auf dem EWG-AKP-Ministerrat in Libreville im Jahre 1982, konnten dann wenigstens 40 Prozent der Forderungen erfüllt werden. ${ }^{110}$

Diese massive Erhöhung der Anträge ergab sich jedoch nicht aus den Änderungen des Systems im Vergleich zu Lomé I, sondern resultierte sowohl aus Naturkatastrophen und der Wirtschaftsrezession (ungünstige Mengenentwicklung) als auch aus der Verschlechterung der Wettbewerbsposition der AKP-Staaten (Preisverfall und Verteuerung der Erdöleinfuhren). ${ }^{111}$

In Lomé IV ist in der Stabex-Regelung neben einigen Verbesserungen angesichts der desolaten finanziellen Situation in den meisten AKP-Staaten auf den Grundsatz der Rückzahlung (Transfer auf Darlehensbasis) verzichtet worden. ${ }^{112}$ Bisher waren nur die am wenigsten entwickelten Länder (LDC), Binnen- und Inselstaaten von dieser Verpflichtung befreit.

${ }^{109}$ Vgl. O.V.: Bonn will die Zusammenarbeit mit den Lomé-Entwicklungsländern fördern, in: BfA/NfA, 22. Februar 1983, O.V.: Stabex, Financing decisions for 1981, in: The Courier, Nr. 76, November - Dezember 1982, S. gelb I; Vergeer, U.: Bericht im Namen des Paritätischen Ausschusses, Teil B: Begründung, Beratende Versammlung AKP-EWG-Sitzungsdokumente 1982 - 1983, 3. November 1982, S. 27; O.V.: ACP memorandum on the Stabex system, in: The Courier, Nr. 79, Mai - Juni 1983, S. 83.

${ }^{110} \mathrm{Vgl}$. Sharma, Y.: Welche Zukunft für Stabex, in: Entwicklung und Zusammenarbeit, Vol. 24, November 1983, S. 6.

11 Vgl. Kommission der EG (Hrsg.): Das EWG-AKP-System zur Stabilisierung der Ausfuhrerlöse, Informatorische Aufzeichnung, P 46, Brüssel, Mai 1983, S. 3.

${ }^{112}$ O.V.: Details on principal developments and innovations, a.a.O., S. 16. 
Während der Laufzeit von Lomé 1 betrug deren Anteil an den gesamten Stabex-Zahlungen bereits 67 Prozent. ${ }^{113}$

Im Lome-IV-Abkommen ist der Stabex-Fonds um mehr als 60 Prozent gegenüber Lomé III auf 1,5 Mrd. ECU erhöht worden, während der Laufzeit von Lomé II betrug er lediglich 557 Mio. ECU (s. Tab. 1).

Bei einem Vergleich der Stabex-Verfahren in den einzelnen Lome-Abkommen werden die Erfahrungen aus der fünfzehnjährigen AKP-EG-Zusammenarbeit deutlich:

Während der Laufzeit von Lomé I und Lomé II war der Empfängerstaat bei der Verwendung der Stabex-Darlehen nicht an Auflagen gebunden, abgesehen davon, daß die Mittel in dem betroffenen Sektor oder zur Förderung der Diversifizierung in anderen Sektoren eingesetzt werden mußten (Lomé II: Art. 23, Abs. 2). Seit Lomé III ist nun von dem begünstigten AKP-Staat ein Bericht zu erstellen, woraus die Mittelverwendung des jeweiligen Stabex-Transfers zu ersehen ist (Lomé III: Art. 171, Abs. 1). In Lomé IV ist darüber hinaus der EG-Kommission bereits bei der Antragstellung eine umfassende Analyse vorzulegen, unter anderem über den betroffenen Sektor einschließlich der Darlegung der Gründe für die aufgetretenen Verluste sowie eine Planung über die beabsichtigte Mittelverwendung. Sollen die Mittel in einem anderen als den durch Exporterlösrückgänge betroffenen Sektor eingesetzt werden (Diversifikation), sind der EG-Kommission vorab die Gründe darzulegen (Lomé IV: Art. 209). In einem gemeinsam zwischen AKP-Staat und EGKommission erstellten Protokoll wird dann die Mittelverwendung festgelegt. ${ }^{114}$

Von der noch während der ersten Lomé-Abkommen herrschenden Maxime der Europäischen Gemeinschaft, den AKP-Staaten bei der Gestaltung des Mitteleinsatzes der Stabex-Darlehen möglichst freie Hand zu lassen, ist die Europäische Gemeinschaft nach den Erfahrungen der funf. zehnjährigen AKP-EG-Zusammenarbeit wieder abgekommen.

${ }^{113}$ Vgl. Kommission der EG (Hrsg.): Zusammenfassender Bericht ..., a.a.O., S. 1.

114 Vgl. Kommission der EG (Hrsg.): Lomé IV 1990-2000, Hintergrund, Neuerungen, Verbesserungen, in: Europa Infor-mation, Mărz 1990, S. 8. 


\subsubsection{Besondere Finanzierungsfazilität für Bergbauerzeugnisse (Sysmin)}

Leidet ein AKP-Staat unter einem merklichen Rückgang seiner Einkünfte aufgrund interner oder externer Schwierigkeiten bei einem der begünstigten Erzeugnisse Kupfer und Kobalt, ${ }^{115}$ Mangan, Zinn, Phosphate, Bauxit und Aluminiumoxid, Eisenerz sowie seit Lomé IV auch Gold und Uran, sodaß Entwicklungsvorhaben gefährdet sind, kann durch die Regierung des betroffenen AKP-Bergbaustaates bei der EG-Kommission ein Antrag auf Finanzhilfe aus der Sysmin-Fazilität gestellt werden.

Die Voraussetzung dafür ist - ähnlich wie bei der Stabex-Regelung - die Erfüllung bestimmter Kriterien. Dazu gehört die Abhängigkeitsschwelle, derzufolge mindestens. 15 Prozent der gesamten Exporterlöse durch das betreffende Bergbauerzeugnis erwirtschaftet worden sein müssen. Für die am wenigsten entwickelten Länder (LDC), Insel- und Binnenstaaten liegt diese Abhängigkeitsschwelle bei 10 Prozent. Diese Abhängigkeitsschwelle muß seit Lomé III statt in den letzten 4 Jahren vor Antragstellung nur noch in mindestens 2 der letzten 4 Jahre erreicht werden (Lomé III: Art. 180).

Gemäß der Erweiterung des Sysmin-Verfahrens in Lomé III kann auch ein AKP-Staat Mittel beantragen, der mindestens 20 Prozent (LDC: 12 Prozent) seiner Exporterlöse aus der Ausfuhr seiner gesamten mineralischen Erzeugnisse erwirtschaftet hat. Nicht berücksichtigt werden dabei Edelrohstoffe (außer Gold), Erdōl und -gas (Lomé IV: Art. 215 Abs. 1b).

Während der antragstellende AKP-Bergbaustaat im ersten Falle bis zu 35 Prozent der Sysmin-Jahrestranche (Lomé IV: max. 96 Mio. ECU) erhalten kann, werden ihm im zweiten Falle lediglich bis zu 15 Prozent einer Jahrestranche zugebilligt (Lomé IV: Art. 219 Abs. 4).

Eine weitere Voraussetzung für die Zulässigkeit einer Antragstellung sind die Auslösekriterien (s. Abb. 6). Entweder muß ein merklicher, nicht durch den AKP-Staat oder das Unternehmen verschuldeter ${ }^{116}$ Rückgang

${ }^{115}$ Kupfer- und Kobalterzeugnisse werden als Kuppelprodukte behandelt, d.h. es wird ein Rückgang der Gesamtproduktions- oder Gesamtausfuhrkapazität berücksichtigt.

116 Ein solcher Passus wurde erstmals in Lomé IV aufgenommen. 
Abbildung 6: Die Abhängigkeits- und Auslösebedingungen des SysminVerfahrens nach Artikel 215 des Lome-IV-Abkommens

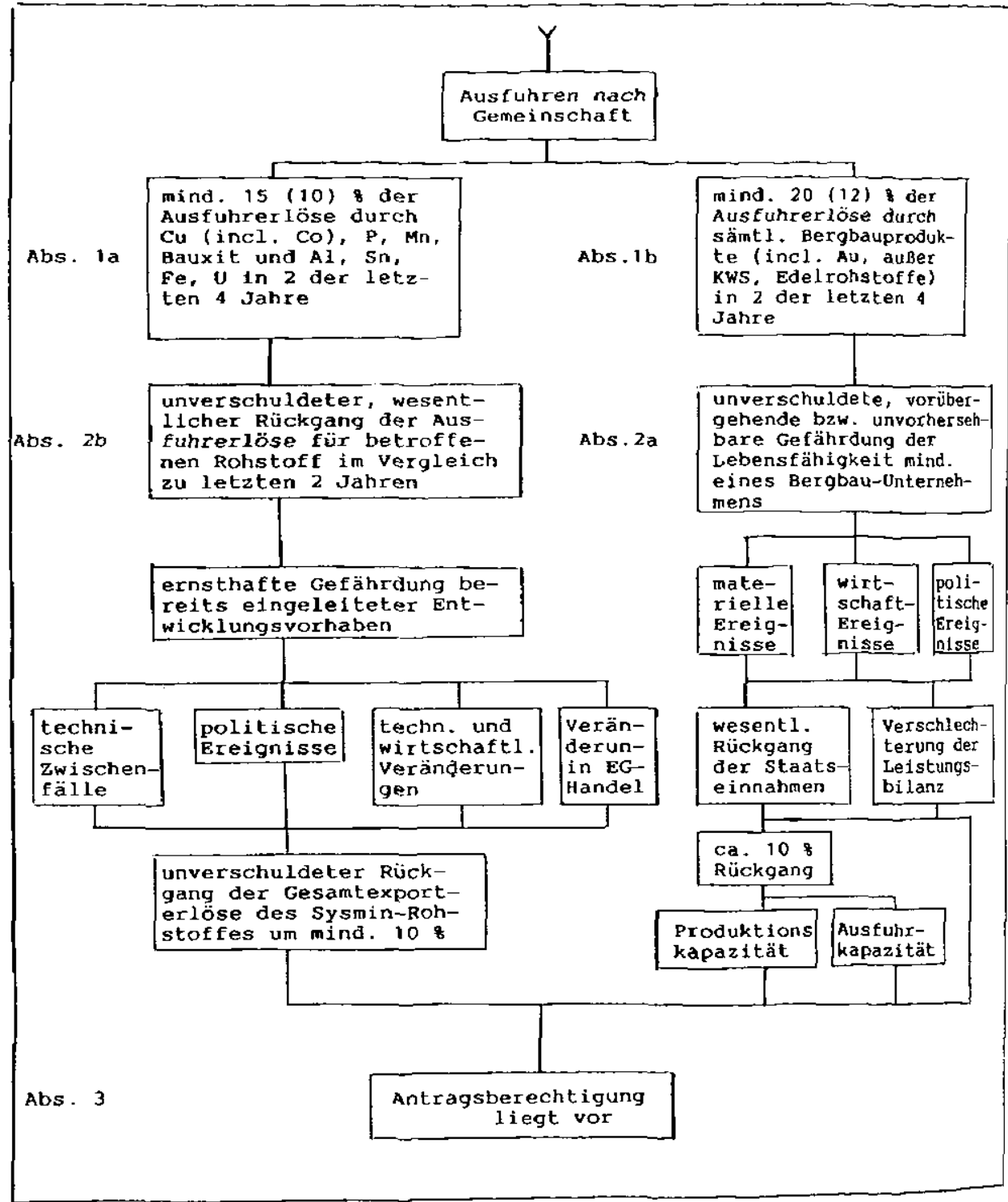

Anm.: Vertikal kumulativ, horizontal fakultativ

Quelle: Eigener Entwurf, vgl. Anhang 4. 
bei den Einkünften des AKP-Staates auftreten, bedingt durch ein Absinken der Produktions- oder Ausfuhrkapazität des jeweiligen Rohstoffes von mindestens 10 Prozent, und/oder es muß eine Verschlechterung der Leistungsbilanz (Lomé III: Rückgang der Exporterlöse) eintreten beziehungsweise eingetreten sein (Lomé IV: Art. 215, Abs. 2a). Im Falle der Abhängigkeit eines AKP-Staates von einem einzelnen Sysmin-Erzeugnis berechtigt ein nicht künstlich hervorgerufener, zehnprozentiger Exporterlösrückgang für das betreffende Produkt, der bereits eingeleitete Entwicklungsvorbaben ernstlich gefährdet, einen Antrag auf Sysmin-Leistungen zu stellen (Lome IV: Art. 215, Abs. 2b).

Anders als beim Stabex-Verfahren ist die Art der Verwendung der Sysmin-Mittel bereits im Text des Sysmin-Verfahrens vorgeschrieben. Erst wenn sich abzeichnet, daß eine wirtschaftlich sinnvolle Wiederherstellung der Produktionsanlagen oder Ausfuhrkapazitäten in dem betroffenen Bergbaubetrieb nicht mehr möglich ist, können die Mittel auch in Alternativ- oder Anschlußprojekten beziehungsweise -programmen Verwendung finden (Lomé IV: Art. 216 Abs. 1). Das automatische Erwachsen von Ansprüchen, wie man es aus dem Stabex-Verfahren kennt, ist in der Sysmin-Regelung nicht vorgesehen. Vielmehr wird die Höhe der bis Lomé III rückzahlbaren Sonderkredite (40 Jahre Laufzeit, 10 Freijahre, 1 \% Zins) - seit Lomé IV Zuschüsse - von der EG-Kommission in Absprache mit dem antragstellenden AKP-Staat festgesetzt. ${ }^{117}$ Dabei werden der Umfang der Schwierigkeiten, die Möglichkeiten der Kofinanzierung und die Pläne zur Sanierung berücksichtigt. Der AKP-Staat leitet die Sysmin-Mittel dann an das betroffene Bergbauunternehmen weiter (Lomé IV: Art. 240 Abs. 1a). Bis einschließlich Lome III wurden die Kredite zu einem gegenüber den Sonderkrediten höheren Zinssatz und einer kürzeren Laufzeit an die Unternehmen weitergeleitet. ${ }^{118}$

Sind aus Sicht eines AKP-Staates die Konditionen der Sysmin-Regelung erfült, stellt dessen Regierung in Zusammenarbeit mit dem betroffenen Unternehmen einen Antrag bei der EG-Kommission in Brüssel. Seit Lomé IV müssen diesem Antrag umfangreiche Zusatzinformationen, über Art

${ }^{117}$ Ende 1991 weist die EG-Kommission in einem Schreiben darauf hin, daß nunmehr die Sysmin-Regelungen von Lomé IV anzuwenden seien; vgl: O.V.: Sysmin, adapting to Lomé IV, in: The Courier, Nr. 131, Januar-Februar 1992, S. gelb VII.

${ }^{118}$ Persönliche Gespräche, Kommission der EG, Brüssel und EG-Delegation, Lusaka, Sambia. 
und Umfang der Schwierigkeiten sowie die geplanten Maßnahmen zur Behebung, beigefügt werden.

Die Kommission überprüft, seit Lome IV anhand einer systematischen technischen, wirtschaftlichen Analyse sowie einer Finanzierungsstudie "in gegenseitigem Einvernehmen" mit dem betroffenen AKP-Staat (Lomé lV: Art. 217 Abs. 2), inwieweit dem Antrag auf Sysmin-Mittel stattgegeben werden kann. Bis einschließlich Lome III handelte es sich hierbei um ein zweistufiges Verfahren, wo vor dem Finanzierungsbeschlub zunächst der Anspruch auf Finanzhilfe festzustellen war. Seit Lome IV erfolgt die Entscheidung in einem einheitlichen Verfahren:

\section{(1) Anwendbarkeit}

- Handelt es sich um ein von der Sysmin-Regelung erfaßtes Erzeugnis? (Lome IV: Art. 215)

- Hat der AKP-Staat zumindest während 2 der 4 vorangegangenen Jahre 15 Prozent seiner Einnahmen aus der Ausfuhr dieses Erzeugnisses bezogen oder 20 Prozent seiner Ausfuhrerlöse aus seinen gesamten Bergbauerzeugnissen, einschließlich Gold und Kohle (ohne sonstige Energie- und Edelrohstoffe) (Art. 215 Abs. 1)?

(2) Zulässigkeit des Antrages

(a) Sind die folgenden Bedingungen (Lomé IV: Art. 215 Abs. 2) erfuillt?

- Das Erzeugnis wird nach der Gemeinschaft ausgeführt;

- Die Produktion ist lebensfähig (Lomé III: Art. 179 Abs. 1b: "und wirtschaftlich");

- Mindestens eine der folgenden Möglichkeiten wird nachgewiesen:

Verschlechterung der Leistungsbilanz;

Festgestellter wesentlicher Rückgang:

- der Produktionskapazität (10 Prozent),

- der Ausfuhrkapazität (10 Prozent), 
- oder (gilt nur für AKP-Staaten mit 15 Prozent Abhängigkeit von einem Sysmin-Erzeugnis) der Ausfuhrerlöse (10 Prozent) und Gefährdung von Entwicklungsvorhaben ;

Voraussehbare Verschlechterung der Leistungsbilanz;

Voraussehbarer wesentlicher Rückgang (in den folgenden Monaten zu erwarten):

- der Produktionskapazität (10 Prozent),

- der Ausfuhrkapazität (10 Prozent).

(b) Die festgestellte oder voraussichtliche Beeinträchtigung der Lebensfähigkeit beziehungsweise der Exporterlösrückgang ist die Folge:

- entweder der Unmöglichkeit, die Produktionsanlagen zu erneuern oder zu erhalten,

- oder schwerwiegender technischer Zwischenfälle,

- oder schwerwiegender innerer oder äuferer politischer Ereignisse,

- technologischer und wirtschaftlicher Änderungen,

- erhebliche Veränderungen im Handel zur Europäischen Gemeinschaft.

(3) Übereinstimmung der Finanzhilfe mit den Zielen der Sysmin-Regelung

- Dies ist nur möglich, wenn die Finanzhilfe den in der Sysmin-Regelung festgehaltenen Zielen entspricht (Lomé IV: Art. 214); sie muß also

- vorübergehender Natur sein,

- Versicherungscharakter haben zugunsten eines AKP-Staates, der unter "von seinem Willen unabhängigen" Störungen leidet,

- ergänzenden Charakter gegenüber "den eigenen Bemühungen des AKPStaates" haben.

- Desweiteren muß die Finanzhilfe für die in der Regelung aufgeführten Malfnahmen (Lomé IV: Art. 216) eingesetzt werden, d.h. für

- Reaktivierungs-, Unterhaltungs- oder Rationalisierungsvorhaben, um die gefährdete(n) Kapazität(en) wieder auf einen wirtschaftlich lebensfähigen Stand zu bringen. Ist das nicht möglich, sind damit Vorhaben zu finanzieren, die die gefährdete(n) Kapazität(en) zumindest teilweise ersetzen. 
Sind diese Bedingungen erfüllt, befindet der Regierungsausschuß über die Höhe der Finanzhilfe. ${ }^{119}$

Neben einem "Versicherungssystem gegen Krisensituationen in den AKPStaaten" sollte die Sysmin-Fazilität ursprünglich auch eine Regelung darstellen, die der EG-Rohstoffpolitik Rechnung trägt. ${ }^{120}$ Dieser Aspekt fällt jedoch immer weniger ins Gewicht wie sich auch an der Entwicklung der Sysmin-Artikel in den einzelnen Lomé-Abkommen (s. Anhang 4) erkennen läßt.

Zusätzlich zu der Sysmin-Regelung ist bei den Verhandlungen zu Lomé Il vereinbart worden, die Entwicklung des Bergbau- und Energiepotentiales in den AKP-Staaten zu unterstützen (Lomé IV: Art. 99 ff. und Art. $105 \mathrm{ff}$.). Hier handelt es sich neben Absichtserklärungen um Instrumente, die - im Gegensatz zum Sysmin-Verfahren - auch außerhalb von wirtschaftlichen Engpässen in Anspruch genommen werden können (s. Kap. 2.5.4).

\subsubsection{Sonstige Instrumente der Lomé-Abkommen}

In den Bereichen finanzielle und technische Zusammenarbeit der LoméAbkommen sind zusätzlich zu den bereits geschilderten noch weitere Instrumente vorgesehen. Dadurch, so beabsichtigten die AKP-EG-Vertragspartner, sollen möglichst viele Sektoren in den AKP-Staaten durch die Lomé-Abkommen profitieren. Die Anzahl der als Kooperations"berechtigt" definierten AKP-Sektoren hat in dem Lomé-IV-Abkommen bisher ihren Höhepunkt erfahren. Unter anderem werden in Lomé IV folgende Kooperationssektoren neu erfaßt:

119 Persönliches Gespräch, Kommission der EG, Brüssel und Bundesministerum für Wirtschaft, Bonn; Vgl. Kommission der EG (Hrsg.): Beschlußfassungsverfahren für die besondere Finanzierungsfazilität für Berg-bauerzeugnisse gemäß Titel II, Kapitel III des Dritten AKP-EWG-Abkommens, Kom (86) 183 endg., Brüssel, 10. April 1986; Bundesrat (Hrsg.): Entwurf eines Gesetzes zu dem Vierten AKP-EWG-Abkommen von Lomé vom 15. Dezember 1989, Bundesratsdrucksache 547/90, Bonn 1990, S. 196.

${ }^{120} \mathrm{Vgl}$. Meyer, K.: Die zweite Konvention von Lomé, a.a.0., S. 15. 
Umwelt, Entwicklung von Handwerk und Weiterverarbeitung, Entwicklung von Unternehmen. Erweitert wurden in Lomé IV unter anderem die Titel Entwicklung der Dienstleistungen sowie Kooperation auf dem Gebiet der Rohstoffe.

Mit Ausnahme der Fonds für Stabex und Sysmin stehen auch in Lomé IV sämtliche Instrumente der Lomé-Zusammenarbeit für die jeweiligen Sektoren zur Verfügung. Das schließt jedoch nicht aus, daß gewisse Sonderinstrumente der finanziellen und technischen Zusammenarbeit nur in einzelnen Sektoren Anwendung finden. Diese Instrumente sind aufgrund der Erfahrungen aus den letzten Lomé-Abkommen in Lomé IV teilweise präzisiert oder modifiziert oder aber auch neu geschaffen worden.

So wurden beispielsweise die Konzepte für das Zentrum für Industrielle Entwicklung (Centre for the Development of Industry, CDI) sowie für das Zentrum für landwirtschaftliche Entwicklung neu gestaltet, nachdem die bisherigen Resultate zeigten, daß die in diese seit Lomé II bestehenden Institutionen gesetzten Erwartungen nicht erfüllt werden konnten. ${ }^{121}$ Im Rahmen der technischen Zusammenarbeit neugeschaffen wurde ein Rohstoff-Ausschuß, dessen Hauptaufgabe die Überprüfung der Anwendungen von Lomé-Maßnahmen für agrarische und mineralische Rohstoffe ist. Darüber hinaus wird dieser Ausschuß bei der Lösung von Fragestellungen im Rahmen der internationalen Zusammenarbeit (z.B. Internationale Rohstoffabkommen) mitarbeiten. ${ }^{122}$

Da sich das Sysmin-Verfahren bis Lomé III auf die Aufrechterhaltung des Status quo beschränkte, wurden den AKP-Staaten durch die Europäische Investitionsbank (EIB) und den Europäischen Entwicklungsfonds (EEF) zusätzlich Mittel zur Entwicklung ihres Bergbau- und Energiesektors zur Verfügung gestellt (s. Tab. 4).

I2I Vgl. Gerth-Wellmann, H.; Kayser, D.: Die industrielle Zusammenarbeit zwischen der EG und den AKP-Staaten, in: AfrikaSpektrum, 15. Jg., 1980/1, S. 48; McQueen, M.: Lomé and industrial co-operation, in: Journal of World Trade Law, 17. Jg., Nr. 6, Dezember 1983, S. 28 f.; O.V. Details on principal developments and innovations, a.a.O., S. 14.

${ }^{122}$ Lome IV: Art. 75 f.; Vgl. O.V.: Details on principal developments and innovations, a.a.O., S. 16. 


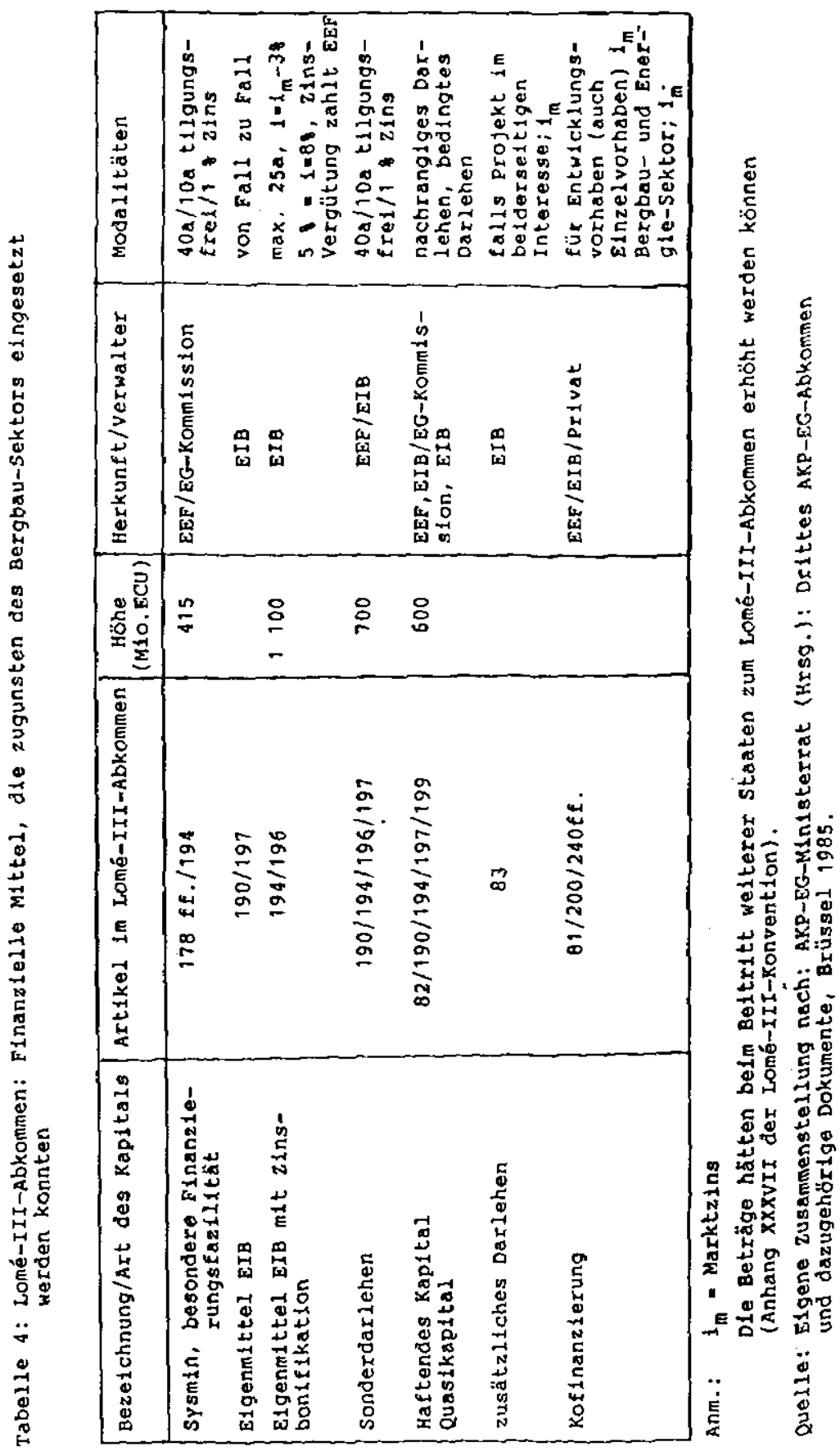


Diese Angebote zur finanziellen aber auch technischen Zusammenarbeit sollten zum einen der schwierigen Lage bei der Energieversorgung vieler AKP-Staaten (hohe Abhängigkeit von Mineralöleinfuhren, Mangel an Brennholz) Rechnung tragen.

Zum anderen sollten die Mittel zum Ausbau der AKP-Bergbauindustrie eingesetzt werden. Zusätzlich zu den entwicklungspolitischen Gesichtspunkten waren die Europäer zu dieser Kooperation bereit, nachdem ihnen bei den Verhandlungen zum Lomé-II-Abkommen der Rückgang der EGInvestitionen in den Entwicklungsländern - speziell im Bergbausektor bewußt geworden war. ${ }^{123}$

Seit Beginn der siebziger Jahre waren die Investitionen der europäischen Bergbaugesellschaften - nach der einsetzenden Unabhängigkeitswelle - in den ehemaligen Kolonien rückläufig, während sie in den Staaten USA, Kanada, Australien und der Republik Südafrika eine deutliche Steigerung erfahren haben ${ }^{124}$ (s. Tab. 5).

Mit Hilfe eines seit Lomé II bestehenden Maßnahmekataloges war beabsichtigt, mehrere Zielgruppen anzusprechen. Zum einen sollte es den nationalen Bergbauunternehmen in den AKP-Staaten leichter gemacht werden, günstige Kredite für kostenintensive Prospektions- und Explorationssvorhaben beziehungsweise für die Aufschluß- und Erweiterungsinvestitionen zu erhalten.

Haftendes Kapital sollte außerdem die Kreditwürdigkeit der AKP-Bergbauunternehmen beziehungsweise der Projekte erhöhen, um so leichter zusätzliche Mittel aus dem Ausland zu mobilisieren. ${ }^{125}$ Auf diese Weise erwartete man, daß die europäischen Bergbaugesellschaften zu verstärkten Investitionen in den AKP-Staaten motiviert werden würden, um auf diese Weise die Konzentration der Aktivitäten auf wenige Bergbauländer zu verringern. ${ }^{126}$

${ }^{123}$ Vgl. Wawrzik, K.: Bericht ..., a.a.O., S. 63; Tetz-laff, R.: Lomé II: "Nachgeschmack ..., a.a.O., S. 13.

${ }^{124}$ Vgl. Rajana, C.: Europe undermined, a.a.O., S. 12.

${ }^{125} \mathrm{Vgl}$. Gerth-Wellmann, H.; Kayser, D.: Die industrielle Zusammenarbeit ..., a.a.O., S. 43.

${ }^{126} \mathrm{Vgl}$. Kommission der EG (Hrsg.): Bericht der Kommission an den AKP-EWG-Ministerrat ..., a.a.O., S. 10. 
Tabelle 5: Die Verteilung und Förderung mineralischer Rohstoffe im Vergleich mit dem finanziellen Engagement europäischer Bergbauunternehmen

\begin{tabular}{|c|c|c|c|c|c|c|c|c|c|}
\hline & \multicolumn{5}{|c|}{$\begin{array}{l}\text { Vorräte mineralischer } \\
\text { Rohstoffe (in Prozent) } \\
\qquad 1981\end{array}$} & \multicolumn{4}{|c|}{$\begin{array}{l}\text { Förderung (in Prozent) } \\
1980\end{array}$} \\
\hline $\begin{array}{l}\text { Indust. - Länder } \\
\text { dayon EG } \\
\text { Staatsh.-Länder } \\
\text { davon AKP/OLG }\end{array}$ & \multicolumn{4}{|c|}{$\begin{array}{r}31,3 \\
2,9 \\
32,7 \\
3,1 \\
\end{array}$} & & \multicolumn{4}{|c|}{$\begin{array}{r}39,3 \\
3,0 \\
33,1 \\
4,4\end{array}$} \\
\hline & \multicolumn{9}{|c|}{$\begin{array}{l}\text { Engagement europ. Berqbaugesellschaften }(*) \\
\begin{array}{l}\text { Prospektions- und Explora- } \\
\text { tions-Ausgaben (in Prozent) }\end{array}\end{array}$} \\
\hline $\begin{array}{l}\text { Indust. -Länder } \\
\text { Entwick.-Länder } \\
\text { davon Afrika } \\
\text { Süd-Amerika } \\
\text { Asien/Ozeanien }\end{array}$ & $\begin{array}{l}1960 \\
60,0 \\
40,0\end{array}$ & $\begin{array}{l}1,970 \\
78,9 \\
21,1\end{array}$ & $\begin{array}{l}1975 \\
84,8 \\
15,2\end{array}$ & $\begin{array}{l}1977 \\
83,1 \\
16,9\end{array}$ & $\begin{array}{l}1980 \\
84,9 \\
15,1 \\
2,8 \\
9,5 \\
2,8\end{array}$ & $\begin{array}{r}1970 \\
38,5 \\
61,5 \\
5,3 \\
4,2 \\
52,0\end{array}$ & $\begin{array}{r}1975 \\
70,4 \\
29,6 \\
13,0 \\
9,8 \\
6,8\end{array}$ & $\begin{array}{l}1977 \\
85,7 \\
14,3 \\
0,4 \\
7,8 \\
6,1\end{array}$ & $\begin{array}{r}1980 \\
81,6 \\
18,4 \\
2,2 \\
15,0 \\
1,2\end{array}$ \\
\hline
\end{tabular}

Quelle: Schmidt, H.; Kruszona, M.: Regionale Verteilung der Weltbergbauproduktion und der Weltvorräte mineralischer Rohstoffe, Bundesanstalt für Geowissenschaften und Rohstoffe (Hrsg.), Hannover 1982, S. 19 und 31; Harms, U. et al.: Die Investitionspolitik der NE-Bergbaugesellschaften, Hamburg 1978, S. 19 u. 60; Kommission der EG (Hrsg.): Communication de la ..., Conseil: Les relations de la CE et les Etats ACP dans le domaine minier, 8. November 1983, Anhang S. 8 - 9.

Vereinbarungsgemäß können im Rahmen einer AKP-EG-Zusammenarbeit bei der Entwicklung des Bergbau- oder Energiepotentiales sämtliche Lomé-Instrumente der technischen und finanziellen Zusammenarbeit eingesetzt werden, gegebenenfalls ist auch der Einsatz anderer Gemein- 
schaftsmittel möglich (Lome IV: Art. 203; Art. 108; Lomé III: Art. 78; Art. 82).

Je nach Art der Projekte - z.B. Forschung und Exploration im Bergbau werden die Anleihen von der Europäischen Investitionsbank (EIB) oder dem Europäischen Entwicklungsfonds (EEF) als Sonderdarlehen, haftendes oder Quasi-Kapital, vergeben (Lomé IV: Art. 234; Lomé III: Art. 199)..$^{127}$

Beim Quasi-Kapital wird zwischen nachrangigen Darlehen - diese Mittel müssen erst zurückgezahlt werden, wenn alle anderen Schuldner befriedigt sind - und Risikokapital - hier kann die Rückzahlung erlassen werden, falls das Projekt erfolglos sein sollte - unterschieden.

Welche der Finanzierungsquellen für eine Maßnahme in Frage kommt, hängt von der Art der zu fördernden Projekte ab beziehungsweise von der Phase, in der sich die Vorhaben befinden. Zur Verdeutlichung der arbeitsteiligen Zuständigkeit werden nachfolgend die Aktionen der EG-Kommission und der EIB im Bergbausektor aufgeführt:

Während die EG-Kommission für die Finanzierungen von Prospektionsvorhaben (u.a. geologische und geophysikalische Untersuchungen, Abschätzung der Vorräte) und Explorationsvorhaben (u.a. Probebohrungen, Schürfarbeiten, gewinntechnische Vorausplanung) zuständig ist, fällt die Finanzierung von vorläufigen Beurteilungen (Pre-Feasibility-Studien) sowohl in den Bereich der EG-Kommission als auch unter die Zuständigkeit der EIB.

Die Ausarbeitung einer Feasibility-Studie sowie die dazugehörigen Tätigkeiten (u.a. Nachweis der Reserven, Abbau- und Aufbereitungstests) werden ausschließlich durch die EIB finanziert. Die Verantwortlichkeit bei der Realisierung von Bergbauvorhaben, d.h. der Finanzierung von Neuoder Erweiterungsinvestitionen liegt hauptsächlich bei der EIB. Der Sys-

${ }^{127} \mathrm{Vgl}$. Kommission der EG/Europäische Investitionsbank (Hrsg.): Die Finanzierung von Projekten zur Erschließung des Bergbau- und Energiepotentiales im Rahmen des 2. Abkommens von Lomé, a.a.O., 1982; Derselbe: Financing of mining projects, in: The Courier, Nr. 109, Mai - Juni 1988, S. 11 f. 
min-Fonds zur Wiederherstellung der Produktions- und Ausfuhrkapazitãten wird allein von der EG-Kommission verwaltet. ${ }^{128}$

Die Aufgaben der technischen Zusammenarbeit beschränken sich hauptsächlich auf Consulting-Leistungen. Dazu gehören Untersuchungen über die Entwicklungs- und Diversifizierungsaussichten sowie die Entsendung von Beratern und Sachverständigen zur Ausführung eines konkreten Auftrages. Bei den zuletzt erwähnten Maßnahmen wird bei gleicher Kompetenz Sachverständigen aus AKP-Staaten der Vorzug gegeben. Diese Vereinbarung soll einem Anliegen der Lomé-Abkommen Rechnung tragen, wonach die Verantwortlichkeit der AKP-Staaten - auch in der technischen Zusammenarbeit - gesteigert werden soll.

Aus Tabelle 6 wird ersichtlich, für welche Vorhaben derartige Darlehen verwendet wurden. Darüber hinaus verdeutlicht dieser Überblick, daß die im AKP-Bergbau gebundenen Mittel der EIB durchaus ein beachtliches Volumen erreichen und von der Größenordnung her zum Teil mit den Finanzierungen aus dem Sysmin-Fonds vergleichbar sind. Für Investitionen im Energie- und Bergbausektor können, sofern an der Realisierung des Vorhabens sowohl ein Interesse des AKP-Staates als auch der Gemeinschaft vorliegt, zusätzliche Darlehen der Europäischen Investitionsbank vergeben werden (Lome IV: Art. 104, Art. 109; Lomé III: Art. 83).

Diese Möglichkeit, über den Rahmen der Lomé-Abkommen hinaus finanzielle Unterstützung zu erhalten, wurde den AKP-Staaten erst in der letzten Verhandlungsphase zum Lomé-II-Abkommen zugestanden. Für diese Vereinbarung wurden in Lome II 200 Mio. ECU in Form von normalen Darlehen, d.h. ohne die sonst übliche 3 Prozent-Zinsvergütung, zur Verfügung gestellt (s. Anhang XXXI des Lome-II-Abkommens: Erklärung der Gemeinschaft zu Art. 59 des Abkommens ${ }^{129}$ ).

${ }^{128} \mathrm{Vgl}$. Kommission der EG/Europäische Investitionsbank (Hrsg.): Die Finanzierung von Projekten zur Erschließung des Bergbau- und Energiepotentiales, a.a.O.; Derselbe: Financing of mining projects, a.a.O., S. $11 \mathrm{f}$.

${ }^{129} \mathrm{Vgl}$. AKP-EWG-Ministerrat (Hrsg.): Zweites AKP-EWG-Abkommen und dazugehörige Dokumente, Brüssel 1981, S. 434. 
Tabelle 6: Finanzierungen der Europäischen Investitionsbank im AKPMineralrohstoffbereich in den Jahren 1981, 1983 und 1989

\begin{tabular}{|c|c|c|c|}
\hline Jahr & staat & $\begin{array}{l}\text { Betrag } \\
\text { lbls Hio. }\end{array}$ & Verwendung \\
\hline 1981 & $\begin{array}{l}\text { Sambia } \\
\text { Gabun } \\
\text { Senegal } \\
\text { Niger } \\
\text { Kenia } \\
\text { Tansania } \\
\text { Malawi } \\
\text { Papua-Neuguinea } \\
\text { Gesamt }\end{array}$ & $\begin{array}{r}33,0 \\
22,0 \\
13,0 \\
10,0 \\
8,0 \\
8,0 \\
1,0 \\
12,0 \\
40,0 \\
147,0\end{array}$ & 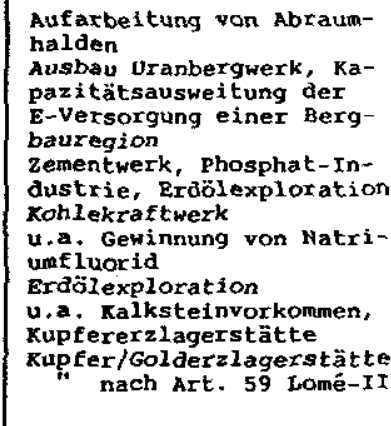 \\
\hline 1983 & $\begin{array}{l}\text { Nigeria } \\
\text { Kamerun } \\
\text { Ghana } \\
\text { Kongo } \\
\text { Malawi } \\
\text { Gabun } \\
\text { Elfenbeinkiuste } \\
\text { Ruanda } \\
\text { Senegal } \\
\text { Sudan } \\
\text { Gesant }\end{array}$ & $\begin{array}{l}10,0 \\
0,5 \\
6,0 \\
1,0 \\
0,5 \\
3,0 \\
+ \\
+ \\
+ \\
+ \\
21,0\end{array}$ & $\begin{array}{l}\text { u.a. kleine und mittlere } \\
\text { Bergbauvorhaben } \\
\text { Etsenerzvorkommen, studie } \\
\text { Investition Manganbergbau } \\
\text { u.a. kleinere und mittler } \\
\text { Bergbauvorhaben } \\
\text { Ralksteinvorkonmen, Studie } \\
\text { Etsenerzvorkommen, Studie } \\
\text { staatl.Betelligung an ze- } \\
\text { nentwerk } \\
\text { studie über diverse Lager } \\
\text { stätten }\end{array}$ \\
\hline 1989 & $\begin{array}{l}\text { Ghana } \\
\text { Ruanda } \\
\text { Burundi } \\
\text { Gesamt }\end{array}$ & $\begin{array}{r}13,0 \\
8,0 \\
4,0 \\
25,0\end{array}$ & $\begin{array}{l}\text { Sanierung von Goldbergwer } \\
\text { ken } \\
\text { fur kleinere Unternehmen } \\
\text { d.a. im Bergbau } \\
\text { Für kletnere Unternehmen } \\
\text { w.a. im Bergbau }\end{array}$ \\
\hline
\end{tabular}

Anm.: +: nicht näher aufgeschlusselt. Es handelt sich vorwiegend um Darlehen aus elgenen Mitteln der Bank und Finanzierungen in Form von haftendem Kapital aus Mitteln des $v$. EEF.

Quedle : Europäische Investitlonsbank (Hrsg.): Jahresbericht 1981 , S. 55 f., Jahresbericht 1982, s. 62 f.. Jahresberlcht 1983, s. 73 f.. Jahresberlcht 1989", S. 93 ff.; 0.V.: European Investment Bank, Breakdown of 1981 investments in the ACP, in: The Courlex, Nr. 73, Mai - Juni 1982, S, $27 \mathrm{f}$. 
Da diese Darlehenskonditionen im Vergleich zu den anderen finanziellen Maßnahmen der Lomé-Abkommen für die AKP-Staaten nicht besonders attraktiv waren, hat während der Laufzeit von Lomé II lediglich PapuaNeuguinea von dieser Finanzhilfe Gebrauch gemacht. Es erhielt im Jahre 1981 von der EIB 52 Mio. ECU für den Abbau goldhaltiger Kupfererze in Ok Tedi (s. Tab. 6). Davon kamen 40 Mio. ECU aus eigenen Mitteln der EIB, gemäß Artikel 59 ohne Zinsvergütung, ${ }^{130} 12$ Mio. ECU wurden in Form von haftendem Kapital vergeben.

Auch bezüglich weiterer spezieller Investitionsvereinbarungen im Bergbau- und Energiesektor (Lome II: Anhang VIII) haben sich die Erwartungen im Hinblick auf eine rege Nutzung durch die AKP-Staaten während der Laufzeit des Lomé-II-Abkommens nicht erfüllt. ${ }^{131}$

\subsubsection{Funktionelle Abgrenzung von Stabex und Sysmin}

In einem nicht unerheblichen Teil der Publikationen über die Lomé-Abkommen wird die Sysmin-Regelung nach wie vor als eine Stabex-Modifikation dargestellt. ${ }^{132}$ Wenn die Sysmin-Regelung in kurzer Form erläutert werden muß, wird auch in jüngeren Veröffentlichungen immer wieder fälschlicherweise das allseits bekannte und bereits in Lomé I etablierte Verfahren zur Stabilisierung der Exporterlöse (Stabex) als Parallele angeführt: Das Sysmin-Verfahren dient der Ausfuhrerlösstabilisierung von Bergbauerzeugnissen. Selbst in EG-Publikationen wird der, nur noch in Ansätzen erkennbare, erlösstabilisierende Aspekt der Sysmin-Fazilität häufig in den Vordergrund gestellt und übergewichtet. ${ }^{133}$

${ }^{130} \mathrm{Vgl}$. O.V.: European Investment Bank, Breakdown of 1981 investments in the ACP, in: The Courier, Nr. 79, Mai - Juni 1982, S. 27; Europäische Investitionsbank (Hrsg.): Jahresbericht 1981, Luxemburg 1982, S. $56 \mathrm{f}$.

${ }^{131}$ Vgl. Grand, B.: Sysmin, in: The Courier, Nr. 79, Mai - Juni 1983, S. $7 \mathrm{f}$.

${ }^{132} \mathrm{Vgl}$. Lingnau, H.: Neue Elemente in der Entwicklungszusammenarbeit der Europäischen Gemeinschaft zu Beginn der 90er Jahre, a.a.O., S. $19 \mathrm{f}$.

${ }^{133}$ Vgl. Kommission der EG (Hrsg.): Lomé IV 1990-2000, a.a.O., S.8. 
Schuld an dieser Tatsache sind zum Teil auch die Formulierungen in den Sysmin-Artikeln der einzelnen Lomé-Abkommen (s. Anhang 4), wo der Begriff "Ausfuhrerlöse" immer wieder zu finden ist.

Bereits im Jahre 1973 hatte die EG-Kommission in einem Memorandum vorgeschlagen, unter anderem auch Kupfererzeugnisse mit in die StabexRegelung einzubeziehen. Durch das Erfassen in dem Stabex-Katalog sollte der Bedeutung dieser Produkte für die Wirtschaft einzelner AKP-Staaten und dem Umfange der Marktinstabilitäten Rechnung getragen werden. Nach einer Abschätzung der aus einer solchen Erweiterung der Stabex-Erzeugnisse erwachsenden Forderungen gegenüber dem Lomé-I-Etat war jedoch deutlich geworden, daß im Lome-I-Abkommen Prioritäten gesetzt werden mußten. Als Konsequenz ist Kupfer aus dem Katalog der StabexProdukte gestrichen worden. ${ }^{134}$ Im September 1978 erklärte die Beratende Versammlung AKP-EWG in einer Entschließung, daß eine Lösung gefunden werden müsse, "um den AKP-Staaten zu helfen, die Probleme des Absatzes von mineralischen Rohstoffen (insbesondere Kupfer, Phosphate) ... zu stabilen und angemessenen Preisen ... zu lösen". ${ }^{135}$

Diese Auffassung wurde auch von dem damaligen Bundeskanzler der Bundesrepublik Deutschland Schmidt unterstützt, nachdem er den afrikanischen Kupfergürtel besucht hatte. ${ }^{136}$

Angesichts der berechtigten Forderungen, auch AKP-Staaten mit einer hohen Exportabhängigkeit von mineralischen Rohstoffen in den LoméAbkommen zu berücksichtigen, wurde bei Beginn der Verhandlungen zu Lomé II seitens der AKP-Staaten vorgeschlagen, das Stabex-Verfahren in dieser Hinsicht zu erweitern. Dieser Position konnte die EG-Verhandlungsdelegation jedoch nicht entsprechen, denn auch im Hinblick auf Lomé II hätte das in der Stabex-Regelung vereinbarte, automatische Erwachsen von Ansprüchen im Falle von Exporterlöseinbußen bei einer Ausweitung auf Bergbauerzeugnisse den Stabex-Etat sehr schnell aufgezehrt. Wäre beispielsweise zusätzlich zu dem bereits erfaßten, mineralischen Rohstoff Eisenerz das Bergbauerzeugnis Kupfer mit in den Katalog aufgenommen worden, hätten, einer Studie zufolge, die in Lomé I zur

${ }^{134} \mathrm{Vgl}$. Kommission der EG (Hrsg.): Zusammenfassender Bericht ..., a.a.O., S. 8.

${ }^{135}$ Ebenda, S. 16.

136 Persönliches Gespräch, Bunđesministerium für Wirtschaft Bonn; Vgl. Rajana, C.: Europe undermined, a.a.O., S. 9. 
Verfügung stehenden Mittel für das Stabex-Verfahren in Höhe von 382 Mio. ECU in etwa verdoppelt werden müssen. ${ }^{137}$

Unrealistisch war diese Schätzung nicht, wurden doch während der Laufzeit des Lomé-I-Abkommens allein für den Erlösausgleich bei Eisenerz 16,4 Prozent der gesamten Stabex-Mittel eingesetzt. ${ }^{138}$

Ein weiterer Grund, Bergbauprodukte nicht im Stabex-Verfahren zu erfassen, waren die Bedenken der Europäischen Gemeinschaft, es könnten aufgrund der oligopolistischen Marktstrukturen - wie sie auf den Märkten mineralischer Rohstoffe vorherrschen - Wettbewerbsverzerrungen eintreten, besonders in den Ländern Asiens und Südamerikas, woher die Europäische Gemeinschaft ebenfalls in erheblichem Maße Bergbauerzeugnisse bezieht $^{139}$ (s. Tab. 16).

Darüber hinaus hielt es die EG-Kommission für erforderlich, die im Vergleich zu agrarischen Rohstoffen anderen Produktions- und Vermarktungsstrukturen bei mineralischen Rohstoffen besonders zu berücksichtigen. Die vertikalen Integrationsstufen von der Erzgewinnung bis hin zu den jeweiligen Weiterverarbeitungsprozessen sind in der Regel stark ausgeprägt. Innerhalb dieser einzelnen Stufen werden von den multinationalen Konzernen häufig interne Verrechnungspreise kalkuliert, die für externe Dritte nicht immer nachvollziehbar sind. Die damaligen Bedenken der EG-Kommission, daß in solchen Fällen Transaktionen manipuliert werden könnten, um ein allein auf Exporterlösen basierendes System zu mißbrauchen, erscheint nicht unbegründet. ${ }^{140}$ In der Sysmin-Regelung des Lome-IV-Abkommens wird diesen Bedenken nochmals explizit Rechnung getragen durch den Passus: "... und nicht künstlich hervorgentfen ..." (Lomé IV: Art. 215, Abs. 2b).

${ }^{137}$ Vgl. Claus, B.: Lomé II, Verhandlungspositionen ..., a.a.O., S. 162. ${ }^{138}$ Vgl. Kommission der EG (Hrsg.): Zusammenfassender Bericht ..., a.a.O., S. 33 und Tabelle 18.

${ }^{139} \mathrm{Vgl}$. Kommission der EG (Hrsg.): Zusammenfassender Bericht ..., a.a.O., S. 42.

${ }^{140} \mathrm{Vgl}$. Rajana, C.: Europe undermined: the Lome response, in: Africa Development, Vol. VI, Nr. 2, April - Juni 1982, S. 10; Kommission der EG (Hrsg.): Zusammenfassender Bericht ..., a.a.O., S. $41 \mathrm{f}$; O.V.: Stabex: Teillösung für das Defizit im Anwendungsjahe 1988, a.a.O., S.4. 
Um dennoch AKP-Staaten mit einem hohen Ausfuhranteil an Bergbauerzeugnissen gegenüber den AKP-Staaten mit Agrarausfuhren, denen das Stabex-System zur Verfügung stand, nicht zu benachteiligen und um die entwicklungspolitische Aussage der Gemeinschaft, wonach "... eine Anhebung der Lebensbedingungen in den AKP-Staaten nur über eine Steigerung der Produktionskapazitäten erreicht werden kann ..."141, zu unterstützen, wurde im Mai 1979 von der EG-Kommission der Vorschlag zur Errichtung einer neuen Fazilität für mineralische Rohstoffe "Système Minière" - genannt Sysmin - unterbreitet. ${ }^{142}$ In diesem Zusammenhang ist anzumerken, daß die offizielle Bezeichnung " Besondere Finanzierungsfazilität für Bergbauerzeugnisse" nicht korrekt ist, da auch Produkte aus weiterführenden Verarbeitungsstufen (z.B. Aluminiumoxid) von der Sysmin-Regelung erfasst werden.

Nachdem sich die AKP-Verhandlungsdelegation im Hinblick auf die Forderung, daß der Begriff "Ausfuhrerlöse" in die Sysmin-Regelung mit aufgenommen werden müsse, durchsetzen konnte, ${ }^{143}$ wurde das SysminKonzept in Anbetracht der kurzen Zeit bis zur Unterzeichnung des LoméII-Abkommens und der engen Verhandlungsspielräume von den AKPStaaten mit der Einstellung: Besser als nichts, akzeptiert. ${ }^{144}$ Damit war die Ausgliederung der mineralischen Rohstoffe aus dem Stabex-Verfahren vollzogen.

Weil das Sysmin-Verfahren aus den oben genannten Überlegungen heraus etabliert wurde, ist die Fazilität häufig im Zusammenhang mit einer Ausfuhrerlösstabilisierung gebracht worden. Da dieser Begriff aus verhandlungstaktischen Gründen auch immer wieder in den jeweiligen Texten der Sysmin-Artikel mehr oder weniger exponiert erscheint, ist bei einer

${ }^{141}$ Irmer, U.: Bericht im Namen des Ausschusses für Entwicklung und Zusammenarbeit über das Umfeld des Nachfolgeabkommens von Lomé II, Teil II, Europa-Parlament Sitzungsdokumente 1983 - 1984, 12. August 1983, S. 135.

142 Vgl. Cavalevu, J.D.V.: Bericht anhand des Jahresberichtes des AKPEWG-Ministerrates für 1982, Teil B: Begründung, Beratende Versammlung AKP-EWG, 13. September 1983, S. 14, Pkt. 24.

${ }^{143}$ Persönliches Gespräch, Bundesministerium für Wirtschaft, Referat EA 6, Bonn.

$144 \mathrm{Vgl}$. Martin, A.: ACP-EEC cooperation in mining, energy and investment, in: The Courier, Nr. 73, Mai - Juni 1982, S. 24. 
nur oberflächlichen Betrachtungsweise eine Parallelisierung zur Exporterlösstabilisierung des Stabex-Verfahrens durchaus verständlich.

Während in den jeweiligen Stabex-Präambeln (Lomé II: Art. 23; Lomé III: Art. 147; Lomé IV: Art. 186) explizit das Ziel der Gewährleistung der Ausfuhrerlösstabilisierung für gewisse Produkte der AKP-Staaten formuliert ist, gehen die Sysmin-Zielsetzungen (Lomé II: Art. 49; Lomé III: Art. 176; Lomé IV: Art. 214) unter anderem davon aus, lediglich eine Unterstützung bei den Bemühungen, einer Verringerung der AKP-Ausfuhrerlöse entgegenwirken, zu leisten.

Deutlich wird der immer geringere Stellenwert der Exporterlösstabilisierung in der Sysmin-Regelung auch bei dem Vergleich der Sysmin-Auslöseschwellen in den jeweiligen Lomé-Abkommen. In Lomé II (Art. 52) und in Lomé III (Art. 179) ist als Auslösekriterium auch ein Rückgang der Exporterlöse vorgesehen, jedoch wurde im Gegensatz zur Stabex-Regelung und zu anderen Sysmin-Auslösekriterien keine Quantifizierung vorgenommen. Andere Kriterien oder ein Von-Fall-zu-Fall-Entscheid der EGGremien regeln das Auslösen des Sysmin-Verfahrens. In Lomé IV (Art. 215 Abs. 2b) nun ist das Auslösekriterium Exporterlösrückgang weiter beschränkt worden, es gilt nur noch für AKP-Staaten, die mindestens 15 Prozent ihrer Exporterlöse aus der Ausfuhr eines der SysminErzeugnisse beziehen. Allerdings wurde erstmalig in der Sysmin-Regelung eine Quantifizierung vorgenommen, die mit 10 Prozent jedoch deutlich über der aktuellen Schwelle der Stabex-Regelung liegt (s. Kap. 2.5.2).

\subsection{Anmerkungen zum Europäischen Entwicklungsfonds (EEF) und der Europäischen Recheneinheit (ECU)}

Der Europäische Entwicklungsfonds (EEF) ist ein Instrument zur Finanzierung der Zusammenarbeit zwischen der Europäischen Gemeinschaft und den Entwicklungsländern, die durch die Lomé-Abkommen mit der Gemeinschaft verbunden sind.

Die EEF-Mittel kommen somit ausschließlich den AKP-Staaten und den Überseeischen Ländern und Gebieten (ÜLG) zugute. 
Während die Lomé-Abkommen durch den IV, bis VII. EEF finanziert wurden bzw. werden, sind die Kooperationsabkommen von Arusha und Jaunde (s. Kap. 2.3) durch Mittel aus dem I. bis III. EEF realisiert worden.

Die Fonds finanzieren sich nicht aus dem EG-Haushalt, sondern werden nach einem speziellen Schlüssel aus den nationalen Etats der EG-Mitgliedsstaaten aufgebracht (s. Tab. 7). Aufgrund dieser Vorgehensweise ist es möglich, die Ausstattung des EEF jeweils für die Laufzeit von fünf

Tabelle 7: Beiträge der EG-Mitgliedsstaaten zu den Europäischen Entwicklungsfonds (EEF)

\begin{tabular}{|l|c|c|c|c|}
\hline & IV. EEF & V. EEF & VI. EEF & VII. EEF \\
\hline & MiO. ECU & Mio. ECU & Mio. ECU & \multicolumn{1}{|c|}{ Mio. ECU } \\
\hline Belgien & 196,87 & 269,35 & 313,45 & 433,23 \\
BR-Deutschland & $\mathbf{8 1 7 , 4 3}$ & 1290,66 & 2062,75 & 2840,48 \\
Dänemark & 75,60 & 114,05 & 164,64 & 227,03 \\
Frankreich & 817,43 & 1172,44 & 1866,45 & 2665,89 \\
Griechenland & - & 74,64 & 98,15 & 133,92 \\
Irland & 18,90 & 27,35 & 43,53 & 60,03 \\
Italien & 378,00 & 524,33 & 995,76 & 1417,77 \\
Luxemburg & 6,30 & 8,35 & 15,04 & 20,74 \\
Niederlande & 250,42 & 337,50 & 446,43 & 609,12 \\
Portugal & - & - & 69,66 & 96,14 \\
Spanien & - & - & 527,17 & 644,99 \\
Vereinigtes & 589,05 & 821,03 & 1312,37 & 1790,64 \\
Königreich & 150,00 & 4639,70 & 7915,40 & 10940,00 \\
\hline Gesamt & 3 & & & \\
\hline
\end{tabular}

Anm.: Angaben gerundet.

Quelle: Eigene Zusammenstellung nach: Persönliches Gespräch BMWI, Bonn 1985; Kommission der EG (Hrsg.): Amtsblatt der EG, Nr. L 24, 29. Januar 1990; BMZ (Hrsg.): Lomé IV, Entwicklungspolitik, Materialien Nr. 82, Bonn (1991), S. 105. 
Jahren (bis Lomé III Laufzeit der Abkommen) festzulegen, wodurch der Disput um jährliche Haushaltsbeiträge umgangen wird. Die Laufzeit des VII. EEF dem Lomé-IV-Abkommen entsprechend auf 10 Jahre zu verlängern, erschien den AKP-EG-Verhandlungspartnern nicht opportun, da in diesem Zeitraum Anpassungen an mittelfristige Entwicklungen nicht möglich wären. Die EG-Kommission verwaltet die Fonds und ist dem AKP-EG-Ministerrat und dem Europäischen Parlament für die ordnungsgemäße Verwendung der Mittel verantwortlich.

Die Europäische Recheneinheit (ERE) löste im Jahre 1975 die alte Recheneinheit der EWG (RE) ab, welche sich noch an der Goldparität des US-Dollars orientierte.

Mit dem Inkrafttreten des Europäischen Währungssystems im März 1979 wurde die ERE als gemeinschaftliche Währungsbasis in allen Tätigkeitsbereichen der Europäischen Gemeinschaft übernommen.

Die Bezeichnung ERE ist seit dem 1. Januar 1981 generell durch die European Currency Unit (ECU) ersetzt worden, wobei die Abkürzung gleichzeitig an die französische Münze "巳̀cu" aus dem 17. Jahrhunderts erinnert.

Die ECU, es handelt sich - ähnlich wie bei der ERE - um eine zusammengesetzte Währungseinheit, wird nach einem vereinbarten Schlüssel aus den Beträgen der einzelnen EG-Währungen gebildet. Dieser Währungskorb setzt sich aus spezifischen Mengen der einzelnen EG-Währungen zusammen, je nach Maßgabe der wirtschaftlichen Bedeutung der einzelnen Mitgliedsstaaten.

Die ECU kann als Basis für das Europäische Währungssystem angesehen werden. Obwohl das Britische Pfund Sterling erst im Oktober 1990 an den Wechselkursmechanismus des Europäischen Währungssystems angeschlossen wurde, gehörte es von Anfang an zum ECU-Korb. Die Griechische Drachme und der Portugiesische Escudo sind noch nicht an den Wechselkursmechanismus angeschlossen. ${ }^{145}$

${ }^{145}$ Vgl. Ypersele, J.; Koeune, J.C.: Das Europäische Währungssystem, Luxemburg 1985, S. 49 f., $60 \mathrm{ff}$. 
Zur Aufstellung des EG-Haushaltes werden immer die Umrechnungskurse des 1 . Februars des vorausgegangenen Jahres verwendet. ${ }^{146}$

Um für die vorliegende Arbeit einheitliche Kurswerte zu erhalten, wurde ERE gleich ECU gesetzt, allgemeine Währungsangaben sind in ECU umgerechnet worden. Soweit nichts anderes vermerkt ist, wurde der jeweils aktuelle Wechselkurs zugrunde gelegt.

\subsection{Weiterführende Aspekte der besonderen Finanzierungsfazilität für Bergbauerzeugnisse (Sysmin)}

Das Schlüsselelement der Sysmin-Regelung liegt seit jeher in der Erhaltung der Produktions- und Ausfuhrkapazitäten (Lomé IV: Art. 214 Abs. 2; Lomé III: Art. 176; Lomé II: Art. 49). Daher ist es unverständlich, weshalb die deutsche Fassung von Lomé IV von der englischen Version insofern abweicht, als sowohl in der Präambel (Art. 214 Abs. 2) als auch in der Definition des Auslösekriteriums des deutschen Textes von "der Produktions- oder Ausfuhrkapazität" gesprochen wird, während in der englischen Fassung von "production or export capacities" die Rede ist.

In den Texten des Lomé-III-Abkommens gab es in diesem Zusammenhang keine Abweichungen.

Während in der Präambel der Sysmin-Regelung in Lomé III die "Kapazität zur Ausfuhr" erwähnt wurde, waren bei der Definition der Sysmin-Auslöseschwelle (Lomé III: Art. 179) "Produktions- oder Ausfuhrkapazitäten" angeführt worden.

Daraus folgte, daß die begriffliche Einheit: "Produktions- oder Ausfuhrkapazitäten" dem zuerst genannten Begriff "Kapazität zur Ausfuhr" subsumiert war. Während sich die untergeordnete Formulierung auf die einzelnen Produktions- und Ausfuhreinrichtungen im betriebswirtschaftlichen Sinne bezog, berücksichtigte der übergeordnete Begriff die Gesamtheit der

${ }^{146}$ Vgl. Kommission der EG (Hrsg.): Der Haushalt der EG, Europäische Dokumentation, 5/1981, Luxemburg 1981, S. 28; Maillet, P.: Die Wirtschaft der EG, Europäische Dokumentation 1-2/1982, Luxemburg 1982, S. 60. 
exportierten Mengen. Im Deutschen wäre der Ausdruck "Kapazität zur Ausfuhr" besser mit "Fähigkeit zur Ausfuhr" übersetzt worden.

Eine weitere Differenzierung ist in den Sysmin-Artikeln von Lome III und Lomé II (Lomé III: Art. 179; Lomé II: Art. 52) vorgenommen worden, wo zwischen den gesamten "Produktions- und Ausfuhrkapazitäten" des betroffenen AKP-Staates - im betriebswirtschaftlichen Sinne - (leur capacité de production ou d'exportation; their capacity to produce or to export) sowie den "Produktionsanlagen und der Ausfuhrkapazität" eines einzelnen Unternehmens (l'outil de production ou de la capacité d'exportation; the production plant or export capacity) unterschieden wurde. Dadurch war deutlich, daß die Sysmin-Fazilität nicht den staatlichen Institutionen der AKP-Staaten zur freien Verfügung steht, sondern als konkrete Projekthilfe primär zur Unterstützung der Modernisierungsund Rehabilitationsanstrengungen der betroffenen Bergbauunternehmen konzipiert wurde. Diese Konzeption ist auch in der neu formulierten Sysmin-Regelung des Lomé-IV-Abkommens durch den Passus: "... die Lebensfähigkeit eines oder mehrerer Unternehmen im Bergbausektor ..." wieder deutlich herausgearbeitet worden (Lomé IV: Art. 215 Abs. 2a).

Die konkrete Unterstützung der betroffenen Bergbaugesellschaften begründete den besonders bei der Einführung des Sysmin-Verfahrens geäuBerten Vorwurf, das Sysmin-Verfahren diene unter dem Deckmantel der Entwicklungszusammenarbeit primär der Rohstoffsicherung der Europäischen Gemeinschaft. Diese Behauptung ist oberflächlich sowie vordergründig und bedarf daher einer differenzierteren Betrachtung.

Es trifft zu, daß der ab Mitte der siebziger Jahre deutliche Rückgang von Explorationen und Investitionen europäischer Bergbaugesellschaften in den AKP-Staaten (s. Tab. 5) der EG-Delegation während der Verhandlungen zu Lomé II die Einwilligung zur Etablierung einer Bergbau-Fazilität erleichterte. ${ }^{147}$ Aus dieser Tatsache jedoch die oben erwähnte Schlubfolgerung zu ziehen, ist falsch.

Bereits die Zusammenstellung der durch Sysmin begünstigten Rohstoffe, wo wesentliche, für die Europäische Gemeinschaft strategische Bergbauerzeugnisse nicht erfaßt sind, sowie die Tatsache, daß allein AKP-Staaten die Aufnahme weiterer Rohstoffe in den Sysmin-Katalog beantragen

$147 \mathrm{Vgl}$. Martin, A.: ACP-EEC cooperation in mining ..., a.a.O., S. 24. 
konnten ${ }^{148}$ (Lomé III: Art. 177 Abs. 2), sind als Indizien für die Nachrangigkeit der EG-Rohstoffsicherung gegenüber entwicklungspolitischen Aspekten anzusehen.

Auf der anderen Seite liegen die positiven Auswirkungen durch die Erhaltung eines Bergbauunternehmens für einen AKP-Staat auf der Hand (s. Kap. 2.3). ${ }^{149}$

Es ist offensichtlich, daß die Sysmin-Regelung von ihrer ursprünglichen Konzeption her speziell auf die beiden AKP-Staaten Sambia und Zaire und deren Probleme im afrikanischen Kupfergürtel zugeschnitten war. Die Konditionen wurden derart festgelegt, daß zur Zeit der Sysmin-Etablierung im Hinblick auf eine Unterstützung der Kupferindustrie (einschl. Kobalt) nur diese beiden AKP-Mitglieder in Frage kamen. ${ }^{150}$

Sambia litt in den Jahren 1975 bis 1986 stark unter den ungünstigen Weltmarktbedingungen und konnte bzw. kann, da ca. 95 Prozent seiner Außenhandelserlöse aus dem Export von Bergbauprodukten (i.w. Kupfer) resultieren, ${ }^{151}$ nicht im erforderlichen Maße notwendigen Investitionen zur Modernisierung des Abbaues, der Aufbereitungsanlagen sowie der Weiterverarbeitung tätigen.

In Zaire ist bei der Union Minière au Haut-Katanga während des KatangaBürgerkrieges aufgrund der politischen Wirrnisse nicht in ausreichendem Maße reinvestiert worden. Nach Beendigung der Unruhen im Jahre 1979 und Verstaatlichung der Minengesellschaft (nunmehr Générale des Carrières et des Mines; Gécamines) wurde zur schnellen Auffüllung der leeren Staatskasse eine rigorose Abbaupolitik betrieben. Wiederum sind die dringend notwendigen Investitionen und Explorationsprojekte nicht durchgeführt worden. Die Konsequenz war ein drastischer Rückgang der Kupfer- und Kobaltproduktion, im Jahre 1979 wurden nur noch $370.000 \mathrm{t}$

${ }^{148}$ Diese Möglichkeit wird den AKP-Staaten in Lomé IV nicht mehr eingeräumt.

149 Vgl. Grand, B.: Sysmin, a.a.O., S. 8.

${ }^{150}$ Persönliche Gespräche, Kommission der EG, Brüssel und Bundesministerium für Wirtschaft, Bonn.

151 Vgl. Pagni, L.: Getting out of the copper straitjacket, in: The Courier, Nr. 85, Mai - Juni 1984, S. 19; Bank of Zambia (Hrsg.): Republic of Zambia, Financial Report, Juni 1984, S. 5; Persönliches Gespräch, Deutsche Botschaft, Lusaka, 1989. 
Kupfer produziert, nachdem es 5 Jahre zuvor noch etwa $500.000 \mathrm{t}$ gewesen waren. Nach Beendigung der Unruhen gelang es, den Bergbau wieder zu rehabilitieren, allerdings nur mit Hilfe massiver internationaler Unterstützung. Im Jahre 1987 wurden wieder etwa 475.000 t Kupfer, $11.000 \mathrm{t}$ Kobalt sowie $55.000 \mathrm{t}$ Zink produziert. ${ }^{152}$

Die anderen in der Sysmin-Regelung erfaßten mineralischen Rohstoffe sind ebenfalls in den Katalog aufgenommen worden, um bestimmten AKPStaaten, die stark vom Export dieser Produkte abhängig sind, die Möglichkeit einzuräumen, ihre Bergbauindustrie zu unterstützen. Um Mauretanien entgegenzukommen, wurden Phosphate berücksichtigt, dem Wunsche weiterer AKP-Staaten entsprechend sind Bauxit (Karibikstaaten), Mangan (Gabun) und Zinn (Ruanda) erfaßt worden, Eisenerze (Liberia, Mauretanien) wurden aus dem Stabex-Verfahren übernommen. In Lomé IV ist darüber hinaus auch Uran in den Sysmin-Katalog aufgenommen worden. Dies erklärt sich aus dem Wunsch der Verhandlungspartner, der AKP-Uranindustrie, welche stark unter den politischen Einflußfaktoren des Uranmarktes leidet, Unterstützung - auch zu Diversifikationsvorhaben - zukommen lassen zu können.

Bei den Verhandlungen zur Sysmin-Regelung in Lomé II und Lomé III ist von den AKP-Staaten der Wunsch geäußert worden, den Sysmin-Katalog auch auf Nickel- und Chromerzeugnisse auszudehnen. ${ }^{153}$ Weshalb jedoch von der Möglichkeit, einen Antrag auf Erweiterung des Kataloges zu stellen (Lomé III: Art. 177 Abs. 2; Lomé II: Art. 50 Abs. 2), kein Gebrauch gemacht wurde, ging aus den Forderungen nicht hervor. In Lomé IV ist ein solcher Passus zur Aufnahme weiterer Bergbauerzeugnisse in das Sysmin-Verfahren nicht mehr enthalten.

Wie bereits ausgeführt, kann seit Lomé III bei der Antragstellung auf Sysmin-Darlehen auch die Abhängigkeit eines AKP-Staates von der Summe seiner gesamten Bergbauerzeugnisse berücksichtigt werden.

152 Persönliches Gespräch, Bundesministerium für Wirtschaft, Bonn 1985; Vgl. O.V.: Shaba and its copper, in: "strong-box" of Zaire, in: The Courier, Nr. 78, März - April 1983, S. 44 f.; Mining Annual Review 1986, S. 417; Gécamines (Hrsg.): Situation actuelle et perspectives, Lumbumbashi, 13. Februar 1988, S. 2.

153 O.V.: Die AKP-Länder verlangen ein besseres NE-System, VWD/NfA, 20. September 1983. 
Daß dabei jedoch einige mineralische Rohstoffe (Kohlenwasserstoffe, Edelmetalle, außer Gold, Diamanten) nicht erfaßt werden, begründet die Europäische Gemeinschaft mit der Konzeption des Sysmin-Verfahrens als Krisenfonds. Danach sollen Bergbauprodukte, deren kontinuierliche Nachfrage weitgehend gesichert ist, nicht durch die Sysmin-Fazilität gestützt werden, deren finanzieller Spielraum ohnehin schon knapp bemessen ist. ${ }^{154}$

Obwohl bisher Sambia insgesamt 83 Mio. ECU und Zaire 81 Mio. ECU als Darlehen aus dem Sysmin-Fonds von Lomé II und Lomé III zugeteilt worden sind (s. Kap. 4.2), gab es angesichts der hohen Zuteilungsquote an nur zwei Begünstigte keinen Widerspruch aus anderen AKP-Ländern.

Die zugebilligten Darlehen sind - wie bereits erwähnt - projektbezogen. In Zusammenarbeit zwischen der EG-Kommission - in nahezu allen AKPStaaten ist eine örtliche EG-Delegation akkreditiert - und dem betroffenen Bergbauunternehmen wird die Mittelverwendung festgelegt. Auf dieser Grundlage werden Bedarfslisten erstellt, welche im Rahmen einer "leicht begrenzten, internationalen Ausschreibung" 155 publiziert werden.

Während der ersten Sysmin-Anwendung für Sambia wurden unter anderem ausgeschrieben: Material zum Austausch von Druckluftrohren, Schaufelradlader, rollendes Material, Gleiswartungsgerăt, Schneckenförderer, Pumpen, Transformatoren, Kabel, Schaltgeräte. Die ersten Ausschreibungen für Zaire betrafen unter anderem: Backenbrecher, schweres Bergbaugerät (5 Löffelbagger), Diesellokomotiven, Radlader, Busse, Lastkraftwagen, Lieferfahrzeuge. ${ }^{156}$

Den Modalitäten entsprechend werden nur Produkte ausgeschrieben, die in Europa oder den AKP-Staaten hergestellt werden. Im Falle Sambias hat man sich, als zum Beispiel Ersatzteile für Bergbauausrüstungen aus anderen Ursprungsländern zu beziehen waren, insofern behelfen können, daß diese Erzeugnisse über die Ausschreibungen der Kofinanzierungen geor-

${ }^{154}$ Persönliches Gespräch, Kommission der EG, Brüssel 1984.

155 Von Ausnahmen abgesehen gelten die Ausschreibungen nur für Unternehmen, die in den Lomé-Unter-zeichnerstaaten niedergelassen sind.

$156 \mathrm{Vgl}$. Kommission der EG (Hrsg.): Supplement zum Amtsblatt der EG, Nr. S 226, 23. November 1982, S. 10 - 13; Ebenda, Nr. S 37, 23. Februar 1983, S. 3 - 5; Ebenda, Nr. S 122, 30. Juni 1982, S. 3 - 7. 
dert wurden (z.B. Darlehen der Weltbank, der Afrikanischen Entwicklungsbank (außer Produkten aus der Republik Südafrika). ${ }^{157}$

In diesem Zusammenhang muß darauf hingewiesen werden, daß die Sysmin-Darlehen nicht nur für bergbautechnische Ausrüstungen oder deren Modernisierung und Instandsetzung eingesetzt werden können. Vielmehr ist es auch möglich, einen Teil der Darlehen zur Unterstützung auf technisch-wirtschaftlichem Gebiet (technische und naturwissenschaftliche Beratung, Marketing, Controlling), Ausbildung sowie für soziale Einrichtungen in den betroffenen Unternehmen (Personentransport, medizinische Einrichtungen) einzusetzen. Unter Lomé II und Lomé III wurden die begünstigten AKP-Regierungen häufiger verpflichtet, die Zinsdifferenz zwischen den Darlehen der Europäischen Gemeinschaft an den AKP-Staat und den Darlehen des Staates an das Unternehmen (s. Kap. 2.4.3) für soziale Zwecke einzusetzen. ${ }^{158}$

Anhand der oben zum Teil aufgeführten, detaillierten Spezifikationen zur Beschaffung von Bergbauausrüstungen wird deutlich, daß man bei der Konzeption des Sysmin-Verfahrens von der in Stabex vertretenen entwicklungspolitischen Maxime - dem einzelnen AKP-Staat die Verantwortung für die Mittelverwendung allein zu überlassen - abgekommen ist. Die zugebilligten Beträge werden in Raten überwiesen, das heißt je nach Stadium und Fortschritt des Projektes beziehungsweise der Lieferungen werden die Mittel transferiert oder die Forderungen der Lieferanten werden von der EG-Kommission aus direkt beglichen.

Die langen Zeiträume von der Antragstellung bis zum Projektbeginn, respektive dem Einsetzen der Lieferung - in der Regel mindestens ein Jahr, im Falle Guayanas sogar über vier Jahre - schränkt die Effizienz der Sysmin-Regelung stark ein. Dies trifft auch dann zu, wenn, wie zum Beispiel im Falle Guayanas, vor der Genehmigung der Sysmin-Tranche ein Sofortkredit in Höhe von ca. 10 Prozent der beantragten Darlehenssumme zur Verfügung gestellt wird.

${ }^{157}$ Persönliches Gespräch, Beratender Ingenieur der ZCCM, Lusaka, Sambia 1989.

${ }^{158}$ Persönliches Gespräch, EG-Delegation, Lusaka, Sambia; ZCCMHeadoffice; Vgl. O.V.: Sysmin, in: The Courier, Nr. 113, Januar Februar 1989, S. XII. 
Die Verzögerungen resultieren in der Regel aus Unzulänglichkeiten bei der Antragsstellung (z.B. Vorlage eines unvollständigen Rehabilitationsprogrammes). Während bis zur Genehmigung des ersten Sysmin-Antrages Sambias viele Monate vergingen, konnte der zweite Antrag innerhalb weniger Wochen genehmigt werden, denn sowohl die Antragsteller aus Sambia als auch die EG-Kommission hatten sich mittlerweile die erforderlichen Kenntnisse angeeignet. ${ }^{159}$ Dennoch vergingen zwischen der Genehmigung dieses Antrages (21. Oktober 1985) und der Unterzeichnung der Darlehensvereinbarung (20. Februar 1986) mehrere Monate. ${ }^{160}$

Wie im Kap. 2.5.3 bereits angedeutet wurde (s. Abb. 6), sollen allein Bergbauunternehmen durch Darlehen aus dem Sysmin-Fonds unterstützt werden können, die vor Eintreten der Schwierigkeiten "lebensfähig" waren (Lomé IV: Art. 215 Abs. 2a).

Auf die Übernahme der in Lomé III noch enger gefaßten Klausel "lebensfähig und wirtschaftlich" (Lomé III: Art. 179 Abs. 1) wurde verzichtet.

Die Einschränkung, nur prinzipiell lebensfähigen Unternehmen Darlehen zu gewähren - eine solche Restriktion sieht das Stabex-Verfahren nicht vor -, basiert auf der Erfahrung, daß unrentable Vorhaben immer weiter gestützt werden müssen, um am Markt bestehen zu können. Darüber hinaus kann es durch solche Maßnahmen zu Überkapazitäten kommen, so daß ein daraus resultierender Preisverfall auch ehedem lebensfähigen Unternehmen in Schwierigkeiten bringen könnte.

Bei Vertretern der europäischen Rohstoffindustrie wird gelegentlich Kritik laut, wenn in ihren Augen diese Klausel eine zu großzügige Interpretation erfährt. Dann wird von den Sysmin-Darlehen als Betriebssubventionen gesprochen, wodurch Bergbauunternehmen in die Lage versetzt würden, auch unrentable Lagerstätten auszubeuten. Dies sei eine potentielle Wettbewerbsverzerrung, welche den Bergbaugesellschaften, die nicht auf die Sysmin-Fazilität zurückgreifen könnten, Schaden zufüge. ${ }^{161}$

${ }^{159}$ Persönliches Gespräch, ZCCM-Headoffice, Lusaka, Sambia 1989.

${ }^{160} \mathrm{Vgl}$. Delegation der Kommission der Europäischen Gemeinschaften (Hrsg.): Co-operation Zambia ..., a.a.O., Vol. 1, S. 43; Vol. 2, S. 78. $161 \mathrm{Vgl}$. Eurometaux (Hrsg.): Lome IV-Sysmin, Comments of the non-ferrons metals industry, a.a.O., S. 1 f.; Vgl. Sames, C.W.: Anaconda, Berichte aus der Rohstoffwelt, München 1986, S. $112 \mathrm{f}$. 
Diese Kritik ist im Ansatz zutreffend. So hat beispielsweise das - unter Lomé III mit über 40 Mio. ECU Sysmin-Darlehen ausgestattete - SelebiPhikwe-Projekt in Botswana seit seiner Produktionsaufnahme im Jahre 1973 kontinuierlich Verluste erwirtschaftet. ${ }^{162}$

Dennoch erscheint es unwahrscheinlich, daß anderen Bergbauunternehmen durch eine großzügige Auslegung der "Lebensfähigkeits"-Klausel direkter Schaden zugefuigt wird. Neben der Tatsache, daß es andere Wettbewerbsverzerrungen gibt, die sich wesentlich gravierender auf die Konkurrenzfähigkeit von Unternehmen auswirken, ermöglicht auch die in der SysminRegelung vorgeschriebene Fall-zu-Fall-Entscheidung eine Abwägung des Umfeldes, welches über betriebswirtschaftliche und nationale Dimensionen hinausgeht.

\subsection{Diskussion und kritische Würdigung der Lomé-Abkommen}

Die seit nunmehr über fünfzehn Jahren bestehenden Lomé-Abkommen haben sowohl positive als auch negative Kritik hervorgerufen. Auf der einen Seite wurden die Lomé-Abkommen glorifiziert, als Modell für Nord-SüdBeziehungen und als Schrittmacher für eine neue Weltwirtschaftsordnung hingestellt. Auf der anderen Seite sind auch negative Stimmen laut geworden. Die Abkommen wurden als Werkzeug des westlichen Imperialismus bezeichnet, es sind die finanziell unzureichende Ausstattung, destabilisierende Effekte der Transferzahlungen sowie das Fördern von Monokulturen durch Stabex bemängelt worden. ${ }^{163}$

Die AKP-Staaten gingen zunächst davon aus, daß für sie mit Beginn des Lome-I-Abkommens eine neue Phase in der Zusammenarbeit mit den

${ }^{162} \mathrm{Vgl}$. Bomsel, O.: Mining and metallurgy investment in the third world; OECD-Development Center Studies, Paris 1990, S. $117 \mathrm{ff}$.

$163 \mathrm{Vgl}$. Bouvier, P.: What to expect from Lomé? Neither the best nor the worst!, in: The Courier, Nr. 113, Januar - Februar 1989, S. 10; St. John, B.: "The groundwork of our continued cooperation in the 1980's", a.a.O., S. 4 - 6; Hermann, R.: On the evaluation of the Stabex-System, in: Intereconomics, Januar - Februar 1982, S. 8 ff.; Wawrzik, K.: Bericht im Namen des Ausschusses für Entwicklung und Zusammenarbeit, a.a.O. 
ehemaligen Kolonialmächten eingeleitet worden sei. Man sprach auch nicht mehr von Assoziations-, sondern von Kooperationsabkommen.

Die anfänglich begeisterte Stimmung über den Abschluß eines derartigen Abkommens hat dann recht schnell einer pragmatischeren Einstellung Platz gemacht. Besonders enttäuscht zeigten sich die AKP-Staaten nach der Unterzeichnung von Lomé II sowohl von den Auswirkungen des Lome-I-Abkommens als auch über die Ergebnisse der Verhandlungen zu dem neuen Abkommen. ${ }^{164}$

Diese Enttäuschung wird verständlich, wenn man berücksichtigt, daß die Entwicklungsländer Ende der siebziger Jahre bereits seit geraumer Zeit, im wesentlichen erfolglos, versuchten, einen eigenen Kurs in die politische und wirtschaftliche Unabhängigkeit zu finden. Mit den Lomé-Abkommen glaubte man dann, eine erfolgversprechende, weil partnerschaftliche Lösung gefunden zu haben, wobei sich jedoch die hochgesteckten Hoffnungen auf einen schnellen Wandel der AKP-Situation als nicht realistisch erwiesen. Häufig sind dann die Gründe für das Nichteintreten der hochgesteckten Erwartungen in den Lomé-Abkommen gesucht worden, zum Beispiel in Vertragspositionen, über welche keine Einigung erzielt werden konnte oder in finanziellen Unzulänglichkeiten der Abkommen.

Anstatt eventuelle Fehler im eigenen System zu suchen, wurde häufig der anderen Vertragspartei die Schuld für ungenügende Fortschritte bei der Entwicklung der AKP-Staaten zugewiesen. Argumente wie: "Die EG muß mehr zahlen", 165 klingen von Seiten der AKP-Staaten durchaus verständlich, besonders auch im Hinblick auf die seit Beginn der neunziger Jahre einsetzende zunehmende Orientierung der Europäischen Gemeinschaft nach Osteuropa. Mit dieser Einstellung allein werden jedoch die Probleme der einzelnen AKP-Staaten nicht behoben. Vielmehr geraten sie auf diese Weise immer tiefer in das Stadium passiver Akzeptanten. Die Erwartungshaltung, die Europäer seien aufgrund der kolonialen Vergangenheit verpflichtet, Zahlungen zu leisten, fördert nicht gerade eigene Anstrengungen in den AKP-Staaten.

Dennoch kann prinzipiell davon ausgegangen werden, daß die Lomé-Abkommen positiv beurteilt werden. Das Abnehmen der negativen Kritik re-

$104 \mathrm{Vgl}$. Twitchett, C.C.: Lomé II: die Enttäuschungen der AKP-Länder, a.a.O., S. $81-88$.

165 Persönliches Gespräch, ZCCM, Sambia, 1989. 
sultiert nicht zuletzt auch daher, daß bisher im fünfjährigen Turnus Neuverhandlungen über das Nachfolgeabkommen abgehalten werden mußten, wobei die Vertragsparteien aus der Erfahrung in der Vergangenheit Optimierungen beziehungsweise Anpassungen an neu entstandene Situationen vornehmen konnten. Dies ist besonders bei den Verhandlungen zu Lomé IV geschehen, wo deutlich Reaktionen auf beziehungsweise Maßnahmen gegen die Verschuldungsproblematik der AKP-Staaten vereinbart worden sind.

Wie aus den Präambeln der jeweiligen Lomé-Abkommen ersichtlich ist, sind die Zielsetzungen der Abkommen sehr ehrgeizig. Dennoch zeigt die Lage in vielen AKP-Staaten, daß die Lomé-Abkommen bisher weder eine treibende Kraft bei der Entwicklung der AKP-Staaten waren, noch einen Katalysator bei der Expansion des AKP-EG-Handels darstellten.

Dies ist allerdings auch nicht das Ziel der Lomé-Abkommen. In den Texten wird immer wieder klargestellt, daß allein die AKP-Staaten für die Festlegung und Umsetzung ihrer eigenen Entwicklungsstrategien verantwortlich sind. Somit dienen die Lomé-Abkommen lediglich zur Unterstützung der Politik der AKP-Staaten. Sie können eine fehlerhafte Entwicklungspolitik der nationalen Regierungen nicht ersetzen. Das heißt mit anderen Worten, daß die in den Abkommen zur Verfügung stehenden Potentiale von den AKP-Staaten ausgeschöpft beziehungsweise aktiviert werden müssen. ${ }^{166}$

Ein Blick in die Statistiken zeigt, daß einige AKP-Staaten die Instrumente und Mittel der Lomé-Abkommen intensiver nutzen beziehungsweise nutzen können als andere AKP-Staaten: Während der Laufzeit des fünften Europäischen Entwicklungsfonds haben beispielsweise 7 Länder in Afrika nahezu ein Drittel der insgesamt bereitgestellten Mittel in Anspruch genommen, 13 von 64 AKP-Staaten haben in dieser Periode über die Hälfte der Mittel beansprucht. ${ }^{167}$ In solchen Fällen sollte seitens der EG-Kommission geprüft werden, ob die "Allokation" der Mittel durch einen systemimmanenten Fehler innerhalb der Abkommen zustande gekommen ist oder, ob es sich hier um wirklich einmalig gravierende Ereignisse innerhalb der durch die Lomé-Mittel begünstigten Länder handelte, so daß die Konzentration der Unterstützungen in diesem Zeitraum notwendig und sinnvoll war. In diesem Zusammenhang wäre auch zu prüfen, inwieweit

$166 \mathrm{Vgl}$. Bouvier, P.: What to expect from Lome?, a.a.O., S. $10 \mathrm{f}$. $167 \mathrm{Vgl}$. ebenda, S. 13. 
AKP-Staaten, die eine erfolgreiche Entwicklungspolitik betreiben, durch andere Konditionen bessergestellt werden sollten als die übrigen AKPStaaten.

Dennoch, trotz der Tatsache, daß die Resultate teilweise hinter den Erwartungen zurückgeblieben sind, handelt es sich bei den Lomé-Abkommen um ein außergewöhnliches, einzigartiges Kooperationsinstrument: Den AKP-Staaten wird durch die Abkommen ein Regelwerk für langfristige, vertraglich fixierte Beziehungen an die Hand gegeben. Neben den bereits erwähnten Interventionsmechanismen, wodurch die AKP-Staaten in die Lage versetzt werden, leichter mit für sie ungünstigen Situationen fertig zu werden, sind besonders die technischen und finanziellen Kooperationsmöglichkeiten sowie die den AKP-Staaten permanent zur Verfügung stehenen Institutionen hervorzuheben. Besonders positiv ist bei der Schwerpunktsetzung von Lome IV zu bemerken, daß zu den in der Regel kurz- und mittelfristig wirkenden Maßnahmen von Lomé I bis Lomé III nun auch langfristige Aspekte stärker berücksichtigt werden. Dazu gehören die Strukturanpassungsmaßnahmen, die regionale, das heißt auch länderübergreifende, Zusammenarbeit sowie der Umweltschutz.

Kritisch zu sehen ist die indirekte Verschlechterung der bevorzugten Position der AKP-Staaten durch die zunehmende Öffnung der Europäischen Gemeinschaft gegenüber anderen Entwicklungsländern (s. Kap. 2.2) sowie gegenüber den ehemaligen Staatshandelsländern, besonders in Osteuropa. Dennoch, das Fazit einer Bewertung der Lome-Abkommen ist im Begleittext des Gesetzentwurfes von Lomé IV für den Bundesrat in der Rubrik Alternativen auf einen Nenner gebracht worden: "Keine."168

${ }^{168}$ Bundesrat (Hrsg.): Entwurf eines Gesetzes zu dem Vierten AKP-EWGAbkommen von Lomé IV vom 15. Dezember 1989 sowie zu den mit diesen Abkommen in Zusammenhang stehenden Abkommen; Bundesratsdrucksache 547/80, Bonn 1990, S. 2. 



\section{MINERALROHSTOFFWIRTSCHAFTLICHEN ASPEKTE DER ZUSAMMENARBEIT AKP-EG}

\subsection{Die Versorgung der Europäischen Gemeinschaft mit mine- ralischen Rohstoffen}

Bereits seit Mitte der siebziger Jahre ist die Europäische Gemeinschaft der weltgrößte Importeur von mineralischen Rohstoffen ${ }^{1}$ (s. auch Tab. 8).

Dies begründet sich im wesentlichen aus der erheblichen Nachfrage der in dieser Region agglomerierten Industrie. Hinzu kommt, daß in den Staaten der Europäischen Gemeinschaft ${ }^{2}$ die Erzförderung aufgrund der Erschöpfung der Lagerstätten stark rückläufig ist, woraus ein zunehmender Importbedarf resultiert. ${ }^{3}$

In den achtziger Jahren mußte die Europäische Gemeinschaft etwa 75 Prozent der mineralischen Rohstoffe importieren (s. Tab. 9). Damit befand sich Europa in einer günstigeren Lage als Japan, dessen Rohstoffeinfuhren ungefähr 90 Prozent betrugen, während die USA nur etwa 15 Prozent ihres Rohstoffbedarfes zu importieren hatten. ${ }^{4}$

Der Import von Bergbauprodukten nach der Europäischen Gemeinschaft erfolgte in den achtziger Jahren zu durchschnittlich mehr als 40 Prozent aus westlich orientierten Bergbauländern. 7 mineralische Rohstoffe wurden zu über 50 Prozent, 14 Bergbauprodukte zu mehr als 30 Prozent aus diesen Staaten bezogen. ${ }^{5}$ Die Staatshandelsländer spielten bei der Versorgung der Europäischen Gemeinschaft mit den in Tabelle 4 aufgeführten

1 Vgl. Kommission der EG (Hrsg.): Communication de la Commission au Conseil: Les relations de la Communauté Européene et les Etats ACP dans le domaine minier, Com (83) 651 final, Brüssel, 8. November 1983, S. 1.

${ }^{2}$ Für Spanien und Portugal trifft diese Aussage nur bedingt zu.

${ }^{3}$ Vgl. Saager, R.: Metallische Rohstoffe ..., a.a.O., S. 26.

4 Vgl. Kommission der EG (Hrsg.): Communication ..., a.a.O., S. 7.

${ }^{5}$ Einschließlich der Republik Südafrika; Eigene Berechnungen nach: Statistisches Amt der EG (Hrsg.): Rohstoffe, Versorgung der EG 1975 1983, a.a.O., S. 64 ff.; Derselbe, EC-ACP-Trade, a statistical analysis 1970 - 1981, Luxemburg 1983, S. 403 f. 
Rohstoffen lediglich eine untergeordnete Rolle. ${ }^{6}$. Nur bei 4 Bergbauerzeugnissen lieferten sie im Jahre 1983 mehr als 10 Prozent. $^{7}$

Tabelle 8: Importanteil der Europäischen Gemeinschaft an mineralischen Rohstoffen bezogen auf die Gesamtheit der Industrieländer (in Prozent)

\begin{tabular}{|l|c|c|}
\hline & 1975 & 1981 \\
\hline Phosphate & & \\
Bleikonzentrat & 51,48 & 49,50 \\
Kupferkonzentrat* & 55,79 & 47,89 \\
Zinkkonzentrat & 57,96 & 46,47 \\
Zinnkonzentrat* & 43,93 & 36,17 \\
Eisenerz/-konzentrat & 36,30 & 34,97 \\
Manganerz/-konzentrat & 38,13 & 34,73 \\
Wolframkonzentrat & 27,87 & 33,46 \\
Bauxit, Aluminiumoxid, Aluminium & 37,86 & 27,17 \\
& 30,92 & 25,52 \\
\hline
\end{tabular}

Anm.: *Statistische Ungenauigkeit, hier Reinmetalle; die Angaben aus 1975 und 1981 erklären das EG-Interesse an Rohstoffvereinbarungen bei der Etablierung der Lomé-Abkommen.

Quelle: Eigene Zusammenstellung nach: Statistisches Amt der EG (Hrsg.): EC-ACP Trade, a statistical analysis 1970-1981, Luxemburg 1983, S. $400 \mathrm{f}$.

${ }^{6}$ Im Jahre 1985 betrug der Import metallischer NE-Rohprodukte aus den Staatshandelsländern nach der EG mengenmäßig unter $10 \%$; Eigene Berechnungen nach: Statistisches Amt der EG (Hrsg.): Analytische Übersichten des Außenhandels, NIMEXE, Import 1985, Bd. B, I, Luxemburg 1986.

7 Vanadium 32,3\% (China), Wolfram 21,2 \% (China), Fluor 13,2 \% (China), Nickel 10,1 \% (UdSSR); Vgl. Statistisches Amt der EG (Hrsg.): Rohstoffe ..., a.a.O., S. $64 \mathrm{ff}$. 
Tabelle 9: Importe mineralischer Rohstoffe nach der Europäischen Gemeinschaft 1985 und deren Anteil am Gesamtbedarf

\begin{tabular}{|c|c|c|c|c|c|c|c|c|c|}
\hline \multirow{2}{*}{\multicolumn{2}{|c|}{$\begin{array}{l}\text { mineralischer } \\
\text { Rofstoff }\end{array}$}} & \multicolumn{2}{|c|}{ Menge } & \multicolumn{3}{|c|}{$\begin{array}{l}\text { davon aus } \\
\text { AKP-Staaten*) }\end{array}$} & \multicolumn{3}{|c|}{$\begin{array}{l}\text { Anteil am Gesamtbe- } \\
\text { darf }\end{array}$} \\
\hline & & \multicolumn{5}{|c|}{$\begin{array}{c}\text { Metall- bzw. Phosphatinhalt } \\
\text { (in t) }\end{array}$} & \multicolumn{2}{|l|}{1982} & 1980 \\
\hline Titan & & 564 & 000 & & 15 & 124 & 100 & & 100 \\
\hline zirkon & & 112 & 000 & & & 10 & 100 & $(96)$ & 100 \\
\hline Mangan & 1 & 145 & 000 & & 380 & 826 & 99 & & 100 \\
\hline Niob & & 2 & 270 & & & 0 & 99 & $(89)$ & 98 \\
\hline Vanadium & & 8 & 152 & & & 0 & 95 & & 96 \\
\hline Molybdän & & 8 & 998 & & & 0 & 94 & & 94 \\
\hline Phosphate & 6 & 913 & 000 & & 607 & 766 & 93 & & 90 \\
\hline Kobalt & & 7 & 086 & & 2 & 253 & 93 & $(62)$ & 97 \\
\hline Tantal & & & 220 & & & 31 & 91 & (44) & 92 \\
\hline Chrom & & 526 & 000 & & 49 & 655 & 86 & & 92 \\
\hline Quecksilber & & & 850 & & & 0 & 81 & $(64)$ & 82 \\
\hline Nickel & & 152 & 000 & & 4 & 332 & 80 & & 84 \\
\hline Wolfram & & 5 & 487 & & & 159 & 72 & $(67)$ & 75 \\
\hline Antimon & & 21 & 193 & & & 280 & 71 & & 55 \\
\hline $\operatorname{zinn}$ & & 48 & 926 & & 2 & 350 & 69 & & 78 \\
\hline Kupfer & 1 & 934 & 000 & & 199 & 144 & 66 & & 70 \\
\hline Aluminium & 3 & 704 & 000 & $1:$ & 336 & 635 & 63 & & 74 \\
\hline zink & 1 & 170 & 000 & & 5 & 176 & 58 & & 55 \\
\hline Eisen & 69 & 207 & 000 & 128 & 854 & 381 & 57 & & 58 \\
\hline Blei & & 652 & 000 & & 1 & 715 & 51 & $(4 B)$ & 50 \\
\hline
\end{tabular}

Anm.: Die Angaben in Klammern berücksichtigen den im Jahre 1982 erfolgten Lagerabbau. Im Gegensatz zu der von der EG vorgenommenen Berechnung der ökonomischen Rohstoffimportabhängigkeit wird in der hier angewandten Formel:

Import $\times 100$

$$
\text { Primär- + Sekundärproduktion + Import }
$$

der Lagerabbau nicht benicksichtigt.

*) Mengen ohne Herkunftsnachweis sind nicht berücksichtigt

Quelle: Eigene Berechnungen nach: Statistisches Amt der EG (Hrsg.): EG Rohstoffbilanzen 1980, Luxemburg 1982; Ebenda, 1979-1982, Luxemburg 1985; Derselbe: Rohstoffe, Versorgung der EG, 1975-1983, Luxemburg 1986; Derselbe: Analytische Übersichten des Außenhandels, NIMEXE, Import 1985, Bd. B,H,I, Luxemburg 1986. 
Ein Großteil der nach der Gemeinschaft eingeführten mineralischen Rohstoffe wurde in den achtziger Jahren demzufolge in Entwicklungsländern abgebaut. Dieses Verhältnis wird sich künftig noch weiter zugunsten dieser Staaten verschieben.

Mineralische Rohstoffe, bei denen die Europäische Gemeinschaft eine hohe Einfuhrabhängigkeit aufweist (Importquote über 85 Prozent) sowie einige andere in der Sysmin-Regelung erfaßte Bergbauprodukte, wurden im Jahre 1982 zum überwiegenden Teil aus den westlich orientierten Bergbauländern importiert. Unternehmen in Afrika - ohne die Republik Südafrika - lieferten nur bei 6 lateinamerikanische Produzenten nur bei 5 Rohstoffen einen nennenswerten Importanteil. Die Bergbaugesellschaften in asiatischen Staaten trugen in den achtziger Jahren lediglich bei Zinn und Vanadium im größeren Maße zur EG-Rohstoffversorgung bei (s. Tab. 10).

Der EG-Import von Bergbauprodukten aus AKP-Staaten spielte keine überragende Rolle (s. Tab. 9, Tab. 16). Im Jahre 1985 wurden - von zwanzig betrachteten Rohstoffen - allein Aluminium, Mangan, Wolfram und Kobalt von der Gemeinschaft zu über 30 Prozent aus den AKP-Staaten bezogen. (Im Jahre 1982 betrug der Kobalt-Import aus den AKPStaaten über 60 Prozent.) Drei weitere Rohstoffe sind zu mehr als 10 Prozent aus den AKP-Staaten eingeführt worden. ${ }^{8}$

Durch den EG-Beitritt der beiden iberischen Staaten Spanien und Portugal im Jahre 1986 ergaben sich keine bedeutenden Änderungen in der Rohstoffabhängigkeit der Gemeinschaft. Nur bei Quecksilber (1983: 66 Prozent) und Fluor (1983: 29 Prozent) liefern Unternehmen aus Spanien gröBere prozentuale Anteile nach anderen EG-Staaten, Produzenten aus Portugal lediglich bei Wolfram (1982: 8 Prozent). ${ }^{9}$

So anschaulich die obigen Tabellen den Rohstoffbezug der Europäischen Gemeinschaft als auch die Herkunft der importierten Rohstoffe darlegen, so wenig reichen sie aus, um exakte Aussagen über das Versorgungsrisiko bei einzelnen Rohstoffen für die Europäische Gemeinschaft zu treffen.

${ }^{8}$ Eigene Berechnungen nach: Statistisches Amt der EG (Hrsg.): Analytische Übersichten ..., a.a.O., Derselbe: Rohstoffe ..., a.a.O., S. $64 \mathrm{ff}$.

9 Vgl. Statistisches Amt der EG (Hrsg.): EG-Rohstoffbilanzen 1979 1982, a.a.O., S. 175; Derselbe: Rohstoffe ..., a.a.O., S. 66. 
Tabelle 10: Die Sysmin-Bergbauprodukte und mineralischen Rohstoffe mit hoher EG-Importrate (= 85\%) nach Herkunft 1982 (in Prozent)

\begin{tabular}{|c|c|c|c|c|c|}
\hline Industrieländer & $\begin{array}{c}\underline{\mathrm{Ti}} \\
82,4 \\
(14,8)\end{array}$ & $\begin{array}{c}\underline{\mathrm{Zr}} \\
96,4 \\
(25,1)\end{array}$ & $\begin{array}{l}\left.\underline{M n}^{*}\right) \\
55,7 \\
(38,8)\end{array}$ & $\frac{\mathrm{Nb}}{16,9}$ & $\begin{array}{c}\underline{v} \\
33,2 \\
(16,7)\end{array}$ \\
\hline Afrika & - & - & 20,3 & - & - \\
\hline Asien & - & - & - & - & 15,9 \\
\hline \multirow{2}{*}{$\begin{array}{l}\text { Latein-Amerika } \\
\text { sonst. a) }\end{array}$} & - & - & - & - & - \\
\hline & 17,6 & 3,6 & 24,0 & 83,2 & 50,9 \\
\hline & $\underline{\text { Mo }}$ & $\left.\underline{\underline{p}}_{2} \underline{\underline{O}}_{5}{ }^{\star}\right)$ & $\left.\mathrm{Co}^{\star}\right)$ & $\underline{\mathrm{T}} \mathbf{a}$ & $\underline{\mathrm{Cr}}$ \\
\hline Industrieländer & 69,1 & 20,3 & 15,0 & 45,6 & $\begin{array}{c}42,3 \\
(42,3)\end{array}$ \\
\hline Afrika & - & 52,7 & 63,7 & - & 12,7 \\
\hline Asien & - & - & - & - & - \\
\hline \multirow{2}{*}{$\begin{array}{l}\text { Latein-Amerika } \\
\text { sonst.a) }\end{array}$} & 27,1 & - & - & - & - \\
\hline & 3,8 & 26,9 & 21,3 & 54,3 & 45,0 \\
\hline \multirow{6}{*}{$\begin{array}{l}\text { Industrieländer } \\
\text { Afrika } \\
\text { Asien } \\
\text { Latein-Amerika } \\
\text { sonst. a) }\end{array}$} & $\left.\mathrm{Sn}^{*}\right)$ & $\left.\underline{A 1}^{\star}\right)$ & $\left.\underline{\mathrm{Cu}}{ }^{\star}\right)$ & $\underline{F e^{\star}}$ & \\
\hline & - & 43,0 & $\begin{array}{l}10,8 \\
(6,6)\end{array}$ & 30,6 & \\
\hline & - & 15,8 & 27,2 & 14,9 & \\
\hline & 59,0 & - & - & - & \\
\hline & 21,7 & 10,7 & 19,0 & 24,7 & \\
\hline & 19,3 & 30,5 & 43,0 & 29,8 & \\
\hline
\end{tabular}

Anm.: Die Angaben in Klammern beziehen sich auf Importe aus der Republik Südafrika

a) Einschl. Mengen ohne Herkunftsnachweis.

*) Im Sysmin-Katalog erfaßte Rohstoffe.

Quelle: Eigene Zusammenstellung nach: Statistisches Amt der EG (Hrsg.): Rohstoffe, Versorgung der EG 1975 - 1983, Luxemburg 1986, S. $64 \mathrm{ff}$. 
Zwar kann davon ausgegangen werden, daß dem jeweiligen Rohstoff aufgrund seiner hohen Importabhängigkeit ein gewisser Stellenwert in der strategischen Versorgungspolitik beigemessen wird, um jedoch konkrete Aussagen treffen zu können, sind weitere Kriterien erforderlich.

Neben der Einfuhrabhängigkeit zählen dazu:

- die Konzentration der Produzenten und der Vorräte (Anbieterstruktur),

- das politische Umfeld der einzelnen Herkunftsstaaten,

- die Möglichkeiten der Wiedergewinnbarkeit (Recycling) und der Substitution,

- Trends in der wirtschaftlichen Entwicklung.

Weitere Indikatoren - zum Beispiel die Elastizität des Angebotes - präzisieren solche Studien. ${ }^{10}$

Nach einer solchen Untersuchung wird den Stahlveredlern Chrom, Kobalt und Mangan das höchste Ausfallrisiko zugewiesen, gefolgt von Platin, Vanadium und Palladium. Dagegen rangieren die metallischen Rohstoffe Zirkon und Ilmenit (Titan) - sie müssen zu 100 Prozent nach der Europäischen Gemeinschaft importiert werden - nach dem politischen Ausfallrisiko lediglich auf Platz 15 bzw. 16. Von den in der Sysmin-Regelung explizit erfaßten Rohstoffen (s. Kap. 2.5.3) befinden sich 3 Bergbauerzeugnisse - Kobalt, Mangan und Kupfer - unter den ersten 10 Bergbauerzeugnissen mit dem höchsten Ausfallrisiko. Zu den ersten 20 Rohstoffen mit strategischer Bedeutung zählen 2 weitere Sysmin-Rohstoffe: Phosphate und Bauxit/Aluminiumoxid. ${ }^{11}$

$10 \mathrm{Vgl}$. Michaelis, M.: Synthese zum Memorandum über eine Europäische Rohstoffversorgungspolitik, a.a.O., S. 4 f.; Bundesministerium für Wirtschaft (Hrsg.): Mineralische Rohstoffe - Märkte und Perspektiven, Bonn 1982, S. 79 f.; Coplin, W.; O'Leary, M.K.: A systematic approach to political risk analysis for extractive industries, CIM Bulletin, 76, Oktober 1983, S. 52 f.

11 Vgl. Presse- und Informationsamt der Bundesregierung (Hrsg.): Aktuelle Beiträge zur Wirtschafts- und Finanzpolitik Nr. 2/1987: Versorgungslage bei Rohstoffen, Kurzfassung einer Untersuchung im Auftrage des BMWi, Bonn 8. Jan. 1987, S. 31. 
In diesem Zusammenhang sei erwähnt, $\mathrm{da} ß$ auch Lieferengpässe bei mineralischen Rohstoffen, deren Anteil am Gesamthandel nur einen unerheblichen Stellenwert einnimmt, zu weitreichenden Folgen führen können. ${ }^{12}$ Einen Einblick in die weitverzweigte Rohstoffabhängigkeit der weiterverarbeitenden Industrie gibt Anhang 3.

Häufig sind bei den oben erwähnten Risiko-Klassifizierungen Abweichungen festzustellen. Dies erklärt sich aus der Gewichtung der einzelnen Parameter, die je nach Autor, Betrachtungsweise oder Schwerpunkt der Analyse unterschiedlich angesetzt wurde.

Bekanntlich erhält man durch die üblichen Produktionsstatistiken keinen Einblick in die Angebotsstruktur der einzelnen mineralischen Rohstoffe, da die länderbezogenen Statistiken keine Aussagen über die Anbieter und deren Verflechtungen mit den übrigen Marktteilnehmern machen..$^{13} \mathrm{Da}$ in der vorliegenden Arbeit im wesentlichen volkswirtschaftliche Aspekte, bezogen auf einzelne Länder, untersucht werden sollen, ist es in diesem Falle möglich, sich schwerpunktmäßig auf die Produktionsstatistiken zu beschränken.

\subsection{Das Bergbaupotential und die Mineralrohstoffproduktion der AKP-Staaten}

Wie andere Entwicklungsländer müssen auch die AKP-Staaten einen Großteil ihrer Produkte ausführen, um mit den Erlösen sowohl Schulden als auch dringend notwendige Importe wie Nahrungsmittel, Erdölprodukte und Investitionsgüter finanzieren zu können. Zum Beispiel exportierten sie im Jahre 1983 durchschnittlich über 20 Prozent ihres Bruttosozialproduktes. ${ }^{14}$ Diese Situation wird sich auch in absehbarer Zeit nicht verbessern,

12 Vgl. Kürsten, M.: Geographische Verteilung und Rohstofforschung Elemente der Rohstoffsicherung, in: Vortragsveröffentlichungen Haus der Technik, Heft 439, Essen 1981, S. 3; Bundesministerium für Wirtschaft (Hrsg.): Mineralische Rohstoffe, a.a.O., S. 81 f.

$13 \mathrm{Vgl}$. Gocht, W.R.; Zantop, H.; Eggert, R.G.: International mineral economics, a.a.O., S. 134.

${ }^{14}$ Eigene Berechnung nach: Statistisches Amt der EG (Hrsg.): ACP basic statistics 1986, a.a.O., S. 39, 40 und 53. 
zumal - einer UN-Studie zufolge - die landwirtschaftliche Produktion der AKP-Staaten in dem letzten Jahrzehnt nicht in der Lage war, mit dem Bevölkerungswachstum Schritt zu halten. In 26 von 37 untersuchten afrikanischen, aber auch in 4 von 5 karibischen AKP-Staaten war ein Rückgang der Lebensmittelproduktion pro Kopf zu verzeichnen. Nur im pazifischen Raum wurde ein solcher Trend nicht beobachtet. ${ }^{\text {is }}$

Die Unausgewogenheit in den Handelsbilanzen vieler AKP-Staaten erklärt die Bestrebungen der betroffenen Länder - neben einer Importreduzierung und -substitution -, eine Steigerung ihrer Exporte zu realisieren. Von derartigen Maßnahmen wird sowohl eine Konsolidierung der Zahlungsbilanzen als auch eine damit einhergehende Entwicklung des Landes erwartet (s. Kap. 2.1). So wird verständlich, daß den AKP-Staaten - wie auch den anderen Entwicklungsländern - sehr daran gelegen ist, ihre heimischen Ressourcen soweit wie möglich zu aktivieren. Neben der Landwirtschaft (cash-crops) dient besonders die Bergbauindustrie diesem Ziel, obwohl dadurch in der Handelsbilanz auch nachteilige Effekte impliziert werden können, zum Beispiel bei der Beschaffung von Investitionsgütern (s. Kap. 4.8.1).

Nach wie vor ist die Landwirtschaft in den AKP-Staaten der dominierende Sektor, obgleich rückläufige Tendenzen feststellbar sind. In 44 von 48 erfaßten Ländern beschäftigte die Agrarwirtschaft mehr als 20 Prozent der Erwerbstätigen. ${ }^{16}$

Der Bergbausektor dagegen spielt nur bei einigen AKP-Staaten eine ausschlaggebende Rolle. Ende der siebziger Jahre erwirtschaftete die Bergbauindustrie in 16 AKP-Ländern mehr als 10 Prozent des Bruttoinlandproduktes (BIP), in 5 Staaten über 20 Prozent. Hierbei handelte es sich um Kiribati (Phosphate: 43 Prozent), Gabun (Manganerze: 37 Prozent), Trinidad und Tobago (Erdöl: 36 Prozent), Nigeria (Erdöl: 23 Prozent) und Surinam (Bauxit: 21 Prozent) ${ }^{17}$ (s. auch Anhang 2).

15 Statistisches Amt der EG (Hrsg.): EC-ACP-Trade, a.a.O., S. 23 f;; O.V.: Afrika sucht einen Ausweg aus der Krise, in: Blick durch die Wirtschaft, 26. April 1988.

16 Statistisches Amt der EG (Hrsg.): EC-ACP-Trade, a.a.O., S. 21.

17 Ebenda, S. 26. 
Größere Bedeutung für den Welthandel mit mineralischen Rohstoffen besitzen lediglich 21 der 69 AKP-Staaten. ${ }^{18} \mathrm{Zu}$ den AKP-Staaten, die als "Land mit herausragendem Geopotential" bezeichnet werden können, zählen Madagaskar, Papua-Neuguinea, Sambia, Simbabwe und Zaire. ${ }^{19}$

Zu den wichtigsten AKP-Bergbauländern gehören: Sambia, Simbabwe und Zaire. Eine nicht unerhebliche Rolle - allerdings in der Regel nur bei einem Rohstoff - spielen: Gabun, Guinea, Guayana, Jamaika, Niger, PapuaNeuguinea, Sierra Leone und $\operatorname{Surinam}^{20}$ (s. Tab. 11).

Die wesentlichen Bergbauprodukte der AKP-Staaten sind: Industriediamanten (1985: über 50 Prozent der Weltproduktion), Bauxit (34 Prozent), Kupfer (17 Prozent) und Kobalt (68 Prozent), Manganerze (11 Prozent), Zinn (2 Prozent) und Uran. ${ }^{21}$ Der AKP-Anteil am Kupferexport sämtlicher Entwicklungsländer beträgt mehr als 30 Prozent, der AKP-Anteil an den Eisenausfuhren der Entwicklungsländer beläuft sich auf etwa 13 Prozent. Bei Bauxit sind es über 40 Prozent. Anfang der achtziger Jahre bezog Sambia etwa 92 Prozent seiner Exporterlöse durch Kupferausfuhren, Zaire etwa 58 Prozent, Papua-Neuguinea etwa 96 Prozent. Im gleichen Zeitraum erwirtschafteten Liberia etwa 63 Prozent seiner Exporterlöse durch die Ausfuhr von Eisenerz, Mauretanien nahezu 55 Prozent. Die AKP-Staaten Guinea, Jamaika, Surinam und Guayana konnten

18 Eigene Berechnungen nach Schmidt, H.; Kruszona, M.: Regionale Verteilung der Weltbergbauproduktion und der Weltvorräte mineralischer Rohstoffe, Bundesanstalt für Geowissenschaften und Rohstoffe (Hrsg.), Hannover 1982, Anhang 2, S. 50 ff.; Statistisches Amt der EG (Hrsg.): EC-ACP-Trade, a.a.O., S. $400 \mathrm{ff}$.

19 Unter dem Geopotential eines Landes versteht man unter anderem die Lagerstätten metallischer und nicht metallischer Rohstoffe, Energieträger, Grundwasser, Böden mit hoher Produktionskapazität, geotechnische Vorteilhaftigkeiten, vgl. Lüttig, G.: Die Entwicklungsländer mit geringem Geopotential, Hannover 1978, S. 8 und 108.

Vgl. Schmidt, H.; Kruszona, M.: Regionale Verteilung ..., a.a.O., S. 29.

21 Die Anteile an der Produktion von Bauxit, Kupfer, Kobalt und Zinn beziehen sich nur auf Länder außerhalb des Ostblocks. Eigene Berechnungen nach: Mining Annual Review 1986. 
zwischen 48 Prozent und 98 Prozent ihrer Exporterlöse durch Bauxitausfuhren erwirtschaften. ${ }^{22}$

Tabelle 11: Der Rang wichtiger AKP-Staaten unter den Bergbauproduzenten im Jahre 1985

\begin{tabular}{|c|c|}
\hline $\begin{array}{l}\text { Kupfer (a): } \\
\text { Kobalt (a): } \\
\text { Phosphat: } \\
\text { Bauxit (a): } \\
\text { Mangan: } \\
\text { Chrom: } \\
\text { Eisenerz: } \\
\text { Zinn (a): } \\
\text { Nickel (b): } \\
\text { Diamanten: }\end{array}$ & $\begin{array}{l}\text { Zaire (4), Sambia (5), Papua-Neuguinea (11) } \\
\text { Zaire (1), Sambia (2) } \\
\text { Togo (8), Senegal (10) } \\
\text { Guinea (2), Jamaika (4), Surinam (6), } \\
\text { Guayana (8) } \\
\text { Gabun (3) } \\
\text { Simbabwe (5), Madagaskar (10), Sudan (14) } \\
\text { Liberia (10), Mauretanien (14) } \\
\text { Zaire (8), Nigeria (9) } \\
\text { Botsuana (10), Simbabwe (11) } \\
\text { Zaire (1), Botsuana (2), Angola (7), } \\
\text { Ghana (9), Zentralafrikanische Republik (10), } \\
\text { Liberia (11), Sierra Leone (11), } \\
\text { Tansania (13) }\end{array}$ \\
\hline
\end{tabular}

Anm.: Angaben geschätzt

a) ohne Produzenten in Staatshandelsländern

b) Angaben von 1980 .

Quelle: Eigene Zusammenstellung nach: Mining Annual Review 1986; Kommission der EG (Hrsg.): Communication de la Commission au Conseil: Les relations de la CE et les Etats ACP dans le domaine minier, Brüssel, 8. November 1983, S. 15 f.

22 Vgl. ACP-EEC-Assembley Report on Commodities (Hrsg.): Commodity studies, in: The Courier, Nr. 116, Juli - August 1989, S. $76 \mathrm{f}$. 
Die Tatsache, daß weniger als ein Drittel der AKP-Staaten bedeutende Bergbauländer sind, darf nicht zu dem Schluß führen, der Bergbau habe für die Volkswirtschaften der restlichen AKP-Staaten keine Bedeutung. Vielmehr kann die Ausbeutung von Lagerstätten auch in diesen Ländern einen respektablen Beitrag zu den Exporterlösen leisten.

Unter den in Tabelle 12 aufgeführten Rohstoffexporten der AKP-Staaten nach der Europäischen Gemeinschaft sind auch Bergbauerzeugnisse aus Ländern erfaßt, deren Ausfuhren von Bergbauerzeugnissen in der Regel in

Tabelle 12: Die Lieferungen von Bergbauerzeugnissen (außer Energierohstoffen und Edelmetallen) der AKP-Staaten nach der Europäischen Gemeinschaft 1980

\begin{tabular}{|l|r|c|}
\hline & 1 000 ECU & $\begin{array}{c}\text { Anteil an } \\
\text { Importen aus } \\
\text { AKP-Staaten (\%) }\end{array}$ \\
\hline Kupfer, raffiniert & 773240 & 4,03 \\
Eisenerz & 376926 & 1,96 \\
Rohkupfer (Blister) & 199693 & 1,20 \\
Aluminiumoxid & 189972 & 0,99 \\
Bauxit & 139544 & 0,73 \\
Rohaluminium & 127011 & 0,66 \\
Kupferkonzentrat & 124245 & 0,65 \\
Phosphat & 103148 & 0,54 \\
Rohkobalt & 67917 & 0,35 \\
Manganerz, -konzentrat & 46947 & 0,24 \\
Zinn & 29004 & 0,15 \\
Zinnkonzentrat & 27 724 & 0,14 \\
Chromkonzentrat & 6891 & 0,04 \\
Rohblei & 4119 & 0,02 \\
Rohzink & 3190 & 0,02 \\
\hline
\end{tabular}

Quelle: Eigene Berechnung nach: Kommission der EG (Hrsg.): Importations CEE des principaux produits en provenance des Etats ACP 1976-1980, o.O., 3. Dezember 1981, S. V ff. 
überregionalen Betrachtungen keine Berücksichtigung finden, da diese für derartige Statistiken zu unbedeutend sind. Gleichwohl liefern auch diese Exporterlöse einen willkommenen Beitrag zur Verbesserung der Außenhandelsbilanz.

So erzielte im Jahre 1980 Burundi 6,3 Prozent seiner Erlöse aus Exporten nach der Gemeinschaft durch mineralische Rohstoffe (hauptsächlich Diamanten), Kamerun 1,3 Prozent, Lesotho 13,4 Prozent (Diamanten), Madagaskar 3,5 Prozent, die Zentralafrikanische Republik 29,4 Prozent (Diamanten) und Tansania 7,4 Prozent. ${ }^{23}$

Weiterhin sind Exporte von mineralischen Rohstoffen zu erwähnen, die im Vergleich zu dem Hauptbergbauprodukt nur eine untergeordnete Position einnehmen. So lieferte beispielsweise Ruanda im Jahre 1985 (1981) außer Zinn noch 265 (160) t Wolframerze bzw. -konzentrate im Werte von 1,8 $(1,4)$ Mio. ECU nach der Europäischen Gemeinschaft. Diese Ausfuhren erlösten etwa 4 Prozent der Deviseneinnahmen Ruandas aus Exporten nach der Europäischen Gemeinschaft und etwa 2 Prozent der Gesamtexporterlöse. Dennoch finden in den meisten der überregionalen Außenhandelsstatistiken nur die Zinnexporte, Ruandas größte Einnahmequelle unter den mineralischen Rohstoffen, Erwähnung. ${ }^{24}$

Obwohl diese "zweitrangigen" Rohstoffexporte ebenfalls einen Beitrag zur Verbesserung der Leistungsbilanz liefern, erfahren die Produzenten derartiger Rohstoffe, sollten einmal ihre Produktionskapazitäten in Schwierigkeiten geraten, keine Unterstützung durch das Sysmin-Verfahren, da die Exportvolumina nie die erforderliche Abhängigkeitsschwelle erreichen werden (s. Kap. 2.5.3).

Gegenwärtig wird die traditionelle Abhängigkeitsschwelle der Sysmin-Regelung von 14 AKP-Staaten erreicht. Das heißt, die unten aufgeführten Länder beziehen 15 Prozent ihrer Ausfuhrerlöse durch den Export eines der Sysmin-Bergbauprodukte und könnten - falls ein etwa zehnprozentiger

23 Eigene Zusammenstellung nach: Kommission der EG (Hrsg.): Importations CEE des principaux produits en provenance des etats ACP 1976 - 1980, 0.O., 3. Dezember 1981, S. $6 \mathrm{ff}$.

24 Eigene Berechnung nach: Statistisches Amt der EG (Hrsg.): ACP basic statistics 1986, a.a.O., S. 123; Derselbe: Anaytische Übersichten des Außenhandels, NIMEXE, Import 1981, Bd. B, S. 26; Ebenda, 1985, S. 33. 
Rückgang ihrer Produktions- oder Ausfuhrkapazität oder eine Verschlechterung der Außenhandelsbilanz eintritt - einen Antrag auf Mittel aus der Sysmin-Fazilität stellen.

Dies trifft im einzelnen zu für: 25

Kupfer, einschließlich Kobalt:

Sambia, Zaire, Papua-Neuguinea;

Phosphate: Togo, Senegal;

Bauxit, Aluminiumoxid: Ghana, Guinea, Guayana, Jamaika, Surinam; Mangan:

Eisenerz:

Zinn: Gabun;

Liberia, Mauretanien;

Ruanda.

Die seit Lomé III zusätzlich in die Sysmin-Regelung aufgenommene Abhängigkeitsschwelle eröffnet nur vier weiteren AKP-Staaten die Möglichkeit, die Sysmin-Fazilităt zu beanspruchen. Gemäß dieser Erweiterung kann ein AKP-Staat, obwohl er für einzelne Sysmin-Bergbauprodukte die Abbängigkeitsschwelle von 15 Prozent nicht überschreitet, dennoch einen Antrag auf Sysmin-Leistung stellen, wenn mindestens 20 Prozent der Exporterlöse aus der Ausfuhr seiner gesamten Bergbauprodukte (einschließlich Gold, außer sonstiger Edelmetallerze, Erdöl und -gas) bezogen werden (s. Kap. 2.5.3); dies ist zur Zeit nur bei Botsuana, Namibia, Niger und Simbabwe der Fall.

An diesem Länderkatalog wird sich kurz- und mittelfristig keine Änderung einstellen, da in den übrigen 51 AKP-Staaten die Bergbauproduktion - wenn überhaupt - nur langfristig in dem notwendigen Maße gesteigert werden kann, um die geforderten Schwellen zu erreichen.

Von 18 AKP-Staaten mit einer signifikanten Bergbauproduktion (ohne Energierohstoffe) gehören 8 Länder zu den 18 AKP-Staaten, die im Jahre 1981 ein Bruttosozialprodukt von über 2 Mrd. US-\$ erwirtschaften konnten. 9 der AKP-Bergbau-Staaten - sie erfüllen alle das Abhängigkeitskriterium der Sysmin-Regelung - wiesen im Jahre 1981 lediglich ein Bruttosozialprodukt von unter $1,5 \mathrm{Mrd}$. US-\$ auf. ${ }^{26}$

25 Persönliches Gespräch, Kommission der EG, Brüssel.

26 Vgl. O.V.: GNP of ACP-Countries (1981), in: The Courier, Nr. 88, November - Dezember 1984, S. 57. 
In der ersten Hälfte der achtziger Jahre hatten die AKP-Staaten bei den im Sysmin-Katalog erfaßten Rohstoffen nahezu ausschließlich einen Produktionsrückgang hinzunehmen. ${ }^{27}$ Beispielsweise konnte die sambische Kupferproduktion erstmals seit Beginn der achtziger Jahre im Jahre 1986 wieder einen gegenüber dem Vorjahr leichten Produktionsanstieg verzeichnen. Das Niveau der Kupfererzgewinnung des Jahres 1981 wurde nicht wieder erreicht. ${ }^{28}$

Über Produktionsrückgänge hinaus sahen sich die AKP-Staaten in den achtziger Jahren mit einem starken Preisverfall für nahezu sämtliche Rohstoffe konfrontiert. Erst im Jahre 1987 konnten bei einigen Rohstoffen wieder leichte Preisstabilisierungen verzeichnet werden. ${ }^{29}$

Auch die diversen Rohstoffvereinbarungen waren nicht in der Lage, den drastischen Rückgang der Rohstoffpreise zus verhindern oder zu dämpfen. Die AKP-Staaten sind in verschiedenen Rohstoffvereinbarungen vertreten (s. Tab. 13), können aber zumindest vorläufig von diesen Organisationen keine große Unterstützung bei der Lösung ihrer Probleme erwarten.

Auch die Bemühungen auf supranationaler Ebene sind nicht allzu erfolgversprechend. So konnten die Verhandlungspartner bisher immer noch keine Einigung über das Integrierte Rohstoffprogramm, dessen ursprüngliches Konzept auf die Vierte UNCTAD-Konferenz im Jahre 1976 zurückgeht, erzielen. Das Abkommen über den im Jahre 1980 ins Leben gerufenen "Gemeinsamen Fonds für Rohstoffe" - das wesentliche Teilstück des Integrierten Rohstoffprogramms - wurde erst im Jahre 1988 von der für ein Inkrafttreten erforderlichen Anzahl von Staaten ratifiziert. Der Gemeinsame Fonds wird jedoch angesichts weiterer erforderlicher Verfahrensabschnitte nicht vor dem Jahre 1992 seine Arbeit aufnehmen. Der sogenannte "Erste Schalter" des Gemeinsamen Fonds, zur Finanzierung von Rohstoffabkommen mit Ausgleichslagern, wird nach jetzigem Stand wohl kaum in Aktion treten, da es an der ausreichenden Anzahl derartiger Rohstoffabkommen fehlt. Darüber hinaus spielen die mineralischen Rohstoffe in dem UN-Programm nur eine untergeordnete Rolle: Unter den

27 Vgl. Kommission der EG (Hrsg.): Communication ..., a.a.O., S. $15 \mathrm{f}$.

Vgl. Zambia Consolidated Copper Mines (Hrsg.): Annual Report 1987, Lusaka 1987, S. 11; Mining Annual Review, Jahrgänge 1982 bis 1986.

$29 \mathrm{Vgl}$. Fortin, C.: Magnitude and impact of price falls on LCD exporters, in: The Courier, Nr. 116, Juli - August 1989, S. $57 \mathrm{ff}$. 
Tabelle 13: AKP-Staaten als Mitglieder von Rohstoffvereinbarungen

$$
\begin{array}{llll}
\text { Produzentenkartelle } & \\
\text { CIPEC } & \begin{array}{l}
\text { (Kupfer) } \\
\text { IBA }
\end{array} & \begin{array}{l}
\text { (Bauxit) } \\
\text { : Sambia, Zaire, Papua-Neuguinea (assoziiert) } \\
\text { Dominikanische Republik, Ghana, Guinea, } \\
\text { Guayana, Jamaika, Sierra Leone, Surinam }
\end{array} \\
\text { APEF } & \text { (Eisenerz) } & \begin{array}{l}
\text { Liberia, Mauretanien, Sierra Leone; Swasiland } \\
\text { machte bisher von seinem Beitrittsrecht keinen } \\
\text { Gebrauch }
\end{array}
\end{array}
$$

Produzentenvereinigungen

PTA (Wolfram) : Ruanda, Zaire

CIMNF (Kobalt) : Sambia, Zaire

UI (Uran) : Gabun, Namibia

Internationale Rohstoffabkommen

ITA (Zinn)

bis 1989 : Nigeria, Zaire

Quelle: Eigene Zusammenstellung nach: Gocht, W.; Zantop, H.; Eggert, R.G.: International Mineral Economics, Berlin/Heidelberg/New York/Tokyo, S. 150 ff.; Jütte-Rauhut, J.: Internationale Marktregulierungen als Risikofaktor bei Projektfinanzierungen im Bergbau, Diss. RWTH Aachen, Internationale Kooperation, Bd. 35, Baden-Baden 1987, S. 191, 234, 246 ff.

10 "core commodities", deren Preise mittels Bufferstocks gestützt werden sollen, befinden sich nur Kupfer und Zinn. Die weiteren - auf insgesamt 18 Rohstoffe zutreffenden - Maßnahmen jedoch würden auch Manganerz, 
Phosphat, Bauxit und Eisenerz berücksichtigen. ${ }^{30}$ Insofern bietet die Sysmin-Regelung der Lomé-Abkommen für die wichtigsten AKP-Bergbaustaaten eine sinnvolle Ergänzung, wenn nicht sogar eine Alternative zu den internationalen Rohstoffvereinbarungen.

\subsection{Der Rohstoffhandel zwischen der Europäischen Gemeinschaft und den AKP-Staaten}

Das Etablieren von Handelsniederlassungen sowie das Gründen von Kolonien in Übersee in den vergangenen Jahrhunderten dokumentierte das starke Interesse der Europäer am Handel mit Rohstoffen, über welche man in Europa selbst nicht verfügte.

Zunächst beschränkte sich das Interesse auf Edelmetalle, Edelsteine und Salz sowie auf agrarische Erzeugnisse (z.B. Gewürze, Genußmittel, Fasern), soweit sie mit den damaligen Fazilitäten transportierbar waren. Mit der industriellen Entwicklung in Europa und dem Aufkommen geeigneter Transportmittel gelangten auch zunehmend Massengüter (z.B. Erze, Konzentrate, Brennstoffe) aber auch agrarische Rohstoffe in erheblichem Umfange in die Industrieländer.

Durch den in den letzten Jahrzehnten immensen Aufschwung des Luftfrachtverkehrs können mittlerweile auch leicht verderbliche, empfindliche und wertvolle Produkte aus nahezu jeder Region der Erde in kurzer Zeit zu ihrem Bestimmungsort transportiert werden.

Diese Entwicklung erklärt die prinzipiell starke Ausweitung des Überseehandels, auch zwischen den AKP-Staaten und der Europäischen Gemeinschaft.

Keine Erklärung liefert die Zunahme der Fracht- und Transportkapazitäten jedoch für die Tatsache, daß sich der Handel mit Produkten von hoher

30 Persönliches Gespräch, Fachvereinigung Auslandsbergbau, Bonn 1989; Vgl. Megzari, A.: The Common Fund for Commodities, in: The Courier, Nr. 116, Juli - August 1989, S. 72 - 75; Gocht, W.R.; Zantop, H.; Eggert, R.G.: International Mineral Economics, a.a.O., S. $198 \mathrm{ff}$;; Laidlaw, K.: The Common Fund: a major break through?, in: ICDA. news, UNCTAD-Bulletin 2. April 1982, S. 2. 
Wertschöpfung zum großen Teil noch auf die Industrieländer und Schwellenländer beziehungsweise auf Exporte nach den Entwicklungsländern beschränkt (s. Abb. 4).

Trotz Bestrebungen in Entwicklungsländern, der entwicklungspolitischen Forderung nach einer verstärkten vertikalen Integration genüge zu leisten, ${ }^{31}$ findet auch heute noch bei einer Vielzahl von Rohstoffen die Weiterverarbeitung nicht an den Produktionsstätten statt. Vielmehr werden die Grundstoffe zunächst auf den internationalen Märkten gehandelt, um darauf in den Verbraucherländern - größtenteils Industrieländern weiterverarbeitet $\mathrm{zu}$ werden. ${ }^{32}$

Prinzipiell lassen sich Rohstoffe in agrarische und mineralische Produkte unterscheiden (s. Tab. 14).

Eine derartige Differenzierung gestattet unterschiedliche Produktions- und Vermarktungsstrukturen zu berücksichtigen, worauf bei entwicklungspolitischen Maßnahmen, wie Aktionen zur Exportförderung, besonders zu achten ist. ${ }^{33}$

Die im Lome-II-Abkommen vorgenommene Ausgliederung der mineralischen Rohstoffe aus der Stabex-Regelung und Erfassung in einer besonderen Finanzierungsfazilität war somit nur die folgerichtige Umsetzung dieser differenzierten Betrachtungsweise.

Besonders deutliche Unterschiede zwischen den agrarischen und mineralischen Rohstoffen treten bei der Produktion auf:

So ist die Gewinnung mineralischer Rohstoffe in der Regel kapitalintensiver als die Produktion von Agrarerzeugnissen, ${ }^{34}$ darüber hinaus

31 Vgl. Fortin, C.: Magnitude and impact of the price falls on LCD exporters, a.a.O., S. 57 - 59; Weltbank (Hrsg.): World Development Report 1983, Washington, D.C. (1983), S. 10.

32 Vgl. Wawrzik, K.: Handel und Märkte, Europa-Parlament, Sitzungsdokumente 1983 - 1984, 12. August 1983, S. 132.

33 Vgl. Hemmer, H.R.: Wirtschaftsprobleme der Entwicklungsländer, a.a.O., S. $513 \mathrm{ff}$.

34 Vgl. Hemmer, H.R.: Wirtschaftsprobleme der Entwicklungsländer, a.a.O., S. 189. 
ist die Erschöpfbarkeit - von Ausnahmen abgesehen ${ }^{35}$ - nur bei Berg. bauprodukten gegeben.

Tabelle 14: Die Unterschiede zwischen mineralischen und agrarischen Rohstoffen

\begin{tabular}{|l|l|}
\hline mineralische Rohstoffe & agrarische Rohstoff \\
\hline engbegrenzte Standortwahl & erheblich erweiterte Standortwahl \\
erschöpfbar & erneuerbar \\
vertikale Integration ausgeprägt & vertikale Integration selten \\
kontinuierliche Produktion & $\begin{array}{l}\text { diskontinuierliche Produktion } \\
\text { (Erntezeit) }\end{array}$ \\
$\begin{array}{l}\text { starke Abhängigkeit der Preise von } \\
\text { zyklischen Angebotsschwankungen } \\
\text { und konjunkturbedingten Nachfra- } \\
\text { geänderungen }\end{array}$ & $\begin{array}{l}\text { starke Abhängigkeit der Preise von } \\
\text { aurre, Kälte, Überschwemmun- } \\
\text { gen }\end{array}$ \\
\hline
\end{tabular}

Quelle: Eigener Entwurf.

Gemeinsam sind beiden Rohstoffkategorien die teilweise erheblichen Erlösschwankungen, die sich sowohl aus Preis- als auch aus Mengenfluktuationen ergeben (s. Abb. 7). Die Bergbauerzeugnisse erfahren eine Beeinflussung îhrer Preise sowohl aufgrund von Änderungen der konjunkturellen Bedingungen in den Wirtschaften der Industrieländer als auch besonders aufgrund von zyklischen Angebotsschwankungen. Während die Rohstoffpreise in den siebziger Jahren beachtliche Steigenungen erfatren haben, trat mit Beginn der achtziger Jahre ein deutlicher Preisverfall ein (s. Abb. 7).

35 Bei den zur Zeit angewendeten Praktiken stellen z.B. tropische Hölzer in der Gruppe der agrarischen Rohstoffe ein nicht erneuerbares Produkt dar. 
Auch nach dem Jahre 1982, als die rezessiven Einflußfaktoren in den OECD-Wirtschaften wieder deutlich in den Hintergrund traten, reduzierten sich die Rohstoffpreisindizes weiter, allein in den Jahren 1984 - 1986 über 20 Prozent (s.Tab. 15).

Abbildung 7: Zusammengefaßte Rohstoffpreisindizes (1970 - 1987)

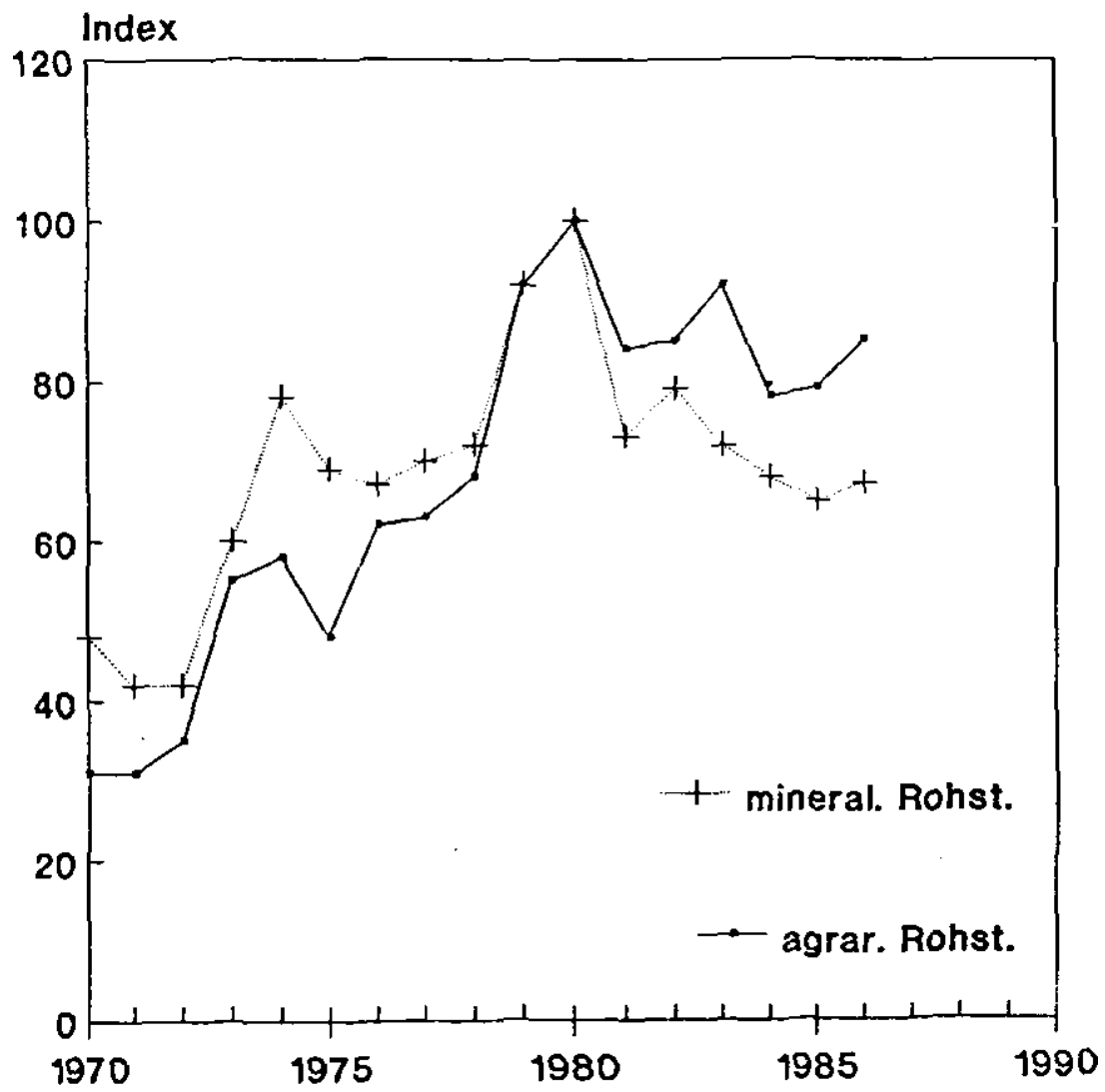

Anm.: *) incl. forstwirtschaftlicher Produkte; Basierend auf Preisen in US-\$; Index $1980=100$

Quelle: Internationaler Währungsfonds (Hrsg.): Primary CommoditiesMarket Developments and Outlook, o.O., Mai 1987. 
Tabelle 15: Rohstoffpreisindizes für wesentliche AKP-Rohstoffe (Basis $1980=100$ )

\begin{tabular}{|l|c|c|c|c|}
\hline & 1981 & 1983 & 1985 & 1987 \\
Rohöl & 111,0 & 86,1 & 84,1 & 51,6 \\
Kaffee & 83,1 & 85,4 & 94,4 & 72,8 \\
Holz & 79,8 & 81,4 & 86,6 & 76,7 \\
Kupfer & 80,0 & 72,1 & 70,4 & K.A. \\
Eisen & 79,8 & 72,9 & 64,9 & 81,5 \\
\hline
\end{tabular}

Anm.: K.A. = Keine Angabe.

Quelle: IMF, International Financial Statistics, in: Stevens, C.: Commodity prices in the 1980s, in: Courier, Nr. 116, Juli - August 1989, S. 60 .

Erst ab dem Jahre 1987 trat auch bei den Rohstoffpreisen eine Wende ein; aufgrund eines schnelleren Preisanstiegs bei Industrieprodukten verschlechterten sich die Terms of Trades für die Rohstoffproduzenten jedoch weiterhin. Auch für die erste Hälfte der neunziger Jahre wird nicht mit einer wesentlichen Erhöhung der Preise für Rohstoffe gerechnet. ${ }^{36}$

Für die meisten AKP-Staaten ist die Europäische Gemeinschaft der wichtigste Handelspartner, sowohl im Hinblick auf Importe als auch auf Exporte. In der Handelsintensität mit der Europäischen Gemeinschaft liegen die AKP-Staaten in den Regionen West- und Zentralafrika sowie Indischer

${ }^{36}$ Vgl. Stevens, C.: Commodity prices in the 1980s: a decade of decline for the ACPs, in: The Courier, Nr. 116, Juli - August 1989, S. 60 f. 
Ozean in der Regel über dem AKP-Durchschnitt (ca. 35 - 40 Prozent), die AKP-Staaten in den Regionen Ostafrika, Karibik und Pazifik darunter. ${ }^{37}$

In den Jahren 1975 bis 1987 nahm der AKP-Anteil an Importen der Europäischen Gemeinschaft aus Entwicklungsländern von 20,5 Prozent auf 16,6 Prozent ab. Dabei ist zu berücksichtigen, daß sich das AKP-ExportVolumen nach der Europäischen Gemeinschaft auf relativ wenige AKPStaaten und Produkte konzentriert. In dem Zeitraum zwischen 1983 und 1987 lieferten die 10 wichtigsten AKP-Lieferanten etwa 70 Prozent der AKP-Exporte nach der Europäischen Gemeinschaft, wobei 10 Hauptprodukte etwa 73 Prozent des AKP-Gesamtexportvolumens nach der Europäischen Gemeinschaft ausmachten. Viele AKP-Staaten exportierten nur ein beziehungsweise wenige Erzeugnisse, lediglich etwa ein Drittel der AKP-Staaten exportieren mehr als 6 Produkte, nur 5 AKP-Staaten mehr als 15 Erzeugnisse. ${ }^{38}$

Die EG-Importe von Bergbauerzeugnissen aus AKP-Staaten und deren prozentualer Anteil an den EG-Gesamtimporten entwickelten sich während der Laufzeit der beiden ersten Lomé-Abkommen (1975 - 1985) unterschiedlich (s. Tab. 16).

Bei den Rohstoffen Kupfer, Kobalt und Zinn waren in dieser Dekade generell rückläufige Einfuhren aus den AKP-Staaten zu verzeichnen. Dies trifft neben dem Liefervolumen auch auf den prozentualen Anteil zu, es mußten folglich - neben einer sinkenden Nachfrage - auch Verluste von Marktanteilen an Produzenten aus Nicht-AKP-Staaten hingenommen werden. Dagegen nahm das Importvolumen in der Periode 1975 - 1980 bei Eisenerzen in geringem, bei Aluminium in größerem Maße zu; der prozentuale Anteil reduzierte sich jedoch. Im Zeitraum 1980 - 1985 änderte sich die Situation, es waren zwar bei diesen Rohstoffen mengenmäBige Einbußen zu akzeptieren, gegenüber den Mitbewerbern aus NichtAKP-Staaten konnten jedoch Marktanteile hinzugewonnen werden. ${ }^{39}$

37 Eigene Berechnungen nach: Statistisches Amt der EG (Hrsg.): Foreign trade: third countries, statistical yearbook 1977 - 1983, a.a.O., S. 26 ff.

38 Vgl. Stevens, C.: Commodity prices in the 1980s: a decade of decline for the ACPs, a.a.O., S. $60 \mathrm{f}$.

39 Eigene Berechnungen nach: Statistisches Amt der EG (Hrsg.): Analytische Übersichten ..., a.a.O.; Derselbe: Rohstoffe ..., a.a.O., S. 64 ff. 
Tabelle 16: Die größten AKP-Lieferanten von Sysmin-Bergbauprodukten nach der Europäischen Gemeinschaft in den Jahren 1975, 1980 und 1983

\begin{tabular}{|c|c|c|c|c|c|c|c|c|c|}
\hline & \multicolumn{3}{|c|}{1975} & \multicolumn{3}{|c|}{1980} & \multicolumn{3}{|c|}{1983} \\
\hline & 11 & $000 \quad t$ & $(8 *)$ & 11 & $000 t)$ & $(8 *)$ & 11 & $000 \mathrm{tl}$ & $(8 *)$ \\
\hline \multicolumn{10}{|l|}{ Aluminium } \\
\hline Guinea & & 419,9 & 11,7 & & 739,2 & 16,6 & & 714,4 & 19,4 \\
\hline Jamaika & & 231,6 & 6,5 & & 278,9 & 6,3 & & 240,4 & 6,5 \\
\hline Surinam & & 238,0 & 6,7 & & 331.7 & 7,5 & & 198,4 & 5,4 \\
\hline \multicolumn{10}{|l|}{ Kupfex } \\
\hline Zaire & & 362,1 & 17,1 & & 332,5 & 14,2 & & 266,4 & 13,8 \\
\hline Sambia & & 370,9 & 17,5 & & 289,4 & 12,4 & & 210,0 & 10,9 \\
\hline \multicolumn{10}{|l|}{ Kobalt } \\
\hline zaire & & 12,9 & 76,0 & & 8,9 & 66,2 & & 1,2 & 16,5 \\
\hline Sambia & & 1,3 & 7,8 & & 1,0 & 7,5 & & 0,8 & 10,7 \\
\hline \multicolumn{10}{|l|}{$\underline{\operatorname{zinn}}$} \\
\hline Zaire & & K. A. & 7,9 & & K.A. & 4.0 & & K.A. & $\mathrm{X} . \mathrm{A}$. \\
\hline Nigeria & & X.A. & 7,9 & & K.A. & 3,7 & & X.A. & R.A. \\
\hline Ruanda & & K.A. & 2,3 & & K.A. & 0,9 & & K.A. & K.A. \\
\hline \multicolumn{10}{|l|}{ Manqan } \\
\hline Gabun & & 321,0 & 20,4 & & 342,0 & 23,7 & & 262,6 & 22,9 \\
\hline Kongo & & K.A. & 0,5 & & K.A. & 3,6 & & K.A. & K.A. \\
\hline \multicolumn{10}{|l|}{ Eisen-Erze } \\
\hline Liberia & $B$ & 232,0 & 11,0 & 8 & 567,0 & 10,0 & 7 & 602,0 & 11,0 \\
\hline Mauritanien & 4 & 271,0 & 5,7 & 4 & 157,0 & 4,9 & 3 & 716,0 & 5,4 \\
\hline \multicolumn{10}{|l|}{ Phosphate } \\
\hline Togo & & K.A. & 11,1 & & K.A. & 12,2 & & K.A. & K.A. \\
\hline Senegal & & X.A. & 9,1 & & K.A. & 5,4 & & K.A. & K.A. \\
\hline
\end{tabular}

Anm.: *) \% der EG-Gesamtimporte K.A.: Keine Angabe

Quelle: Statistisches Amt der EG (Hrsg.): Rohstoffe, Versorgung der EG 1975 - 1983, Luxemburg 1986, S. 64 ff.; Derselbe: EC-ACP Trade, a statistical analysis 1970-1981, Luxemburg 1983, S. $400 \mathrm{ff}$. 
Im Jahre 1985 wurden bei einigen AKP-Bergbauerzeugnissen (Aluminium +13 Prozent, Eisenerze +5 Prozent, Kupfer +4 Prozent) erstmals wieder steigende Importe der Europäischen Gemeinschaft gegenüber dem Vorjahr verzeichnet. ${ }^{40}$ Dabei ist jedoch zu berücksichtigen, daß angesichts der zu dieser Zeit positiven Wirtschaftsentwicklung in den Industrieländern das gesamte Importvolumen an Rohstoffen angestiegen ist, sich der prozentuale AKP-Anteil an den jeweiligen Rohstoffeinfuhren aber nicht wesentlich verändert hat.

Die Europäische Gemeinschaft bezieht die gesamte Exportmenge an AKPEisenerzen und ca. 50 Prozent der AKP-Kupferexporte. ${ }^{41}$

\subsection{Die Situation auf den Märkten der Sysmin-Rohstoffe in den sieb- ziger und achtziger Jahren}

Obwohl die heute bekannten Vorkommen an mineralischen Rohstoffen regional sehr ungleich verteilt sind, nehmen sechs Staaten - die ehemalige UdSSR, USA, Kanada, Australien, die Volksrepublik China und die Republik Südafrika - sowohl in Bezug auf die Anzahl der von ihnen gewonnenen Rohstoffe als auch in Anbetracht ihres Anteils an der Weltproduktion mit über 60 Prozent eine Schlüsselstellung ein. ${ }^{42}$

Bei der Rohstoffproduktion ist die Stellung der Industrieländer stärker als gemeinhin angenommen wird. Dabei ist allerdings zu berücksichtigen, daß in vielen Entwicklungsländern aufgrund der geringen Explorations- und Prospektionstätigkeit noch ein erhebliches, unbekanntes Potential zu erwarten ist. ${ }^{43}$ An der Weltförderung mineralischer Rohstoffe sind 29 Entwicklungsländer mit einem Anteil von über 3 Prozent beteiligt, darunter 9 AKP-Staaten (s. Kap. 3.2). ${ }^{44}$

40 Vgl. Statistisches Amt der EG (Hrsg.): EEC-ACP trade pattern: greater expansion in 1985, a.a.O., S. $58 \mathrm{f}$.

41 ACP-EEC Assembley Report on Commodities (Hrsg.): Commodity studies, a.a.O., S. 76.

42 Vgl. Schmidt, H.; Kruszona, M.: Regionale Verteilung ..., a.a.O., S. 3.

43 Vgl. Kürsten, M.: Geographische Verteilung ..., a.a.O., S. 4.

44 Vgl. Schmidt, H.; Kruszona, M.: Regionale Verteilung ..., a.a.O., S. 3. 
Nach den Energierohstoffen stehen die mineralischen Rohstoffe Eisen und Kupfer auf den Umsatz bezogen an den ersten Positionen, gefolgt von den Phosphaten, den Edel- und den übrigen NE-Metallen und den Stahlveredlern. ${ }^{45}$

Die im Jahre 1972 formulierten Aussagen des "Club of Rome", welche die Erschöpfung von Lagerstätten gewisser Rohstoffe prognostizierten, haben sich nicht bewahrheitet; ${ }^{46}$ zumal die "Erschöpfungs-These" auf falschen Grundannahmen beruhte. Vielmehr riefen die Zunahme der nachgewiesenen Reserven ${ }^{47}$ und der mittelfristige Rückgang der Rohstoffnachfrage $^{48}$ einen Umschwung auf den Rohstoffmärkten hervor. Aus der Sorge vor einer fortschreitenden Verknappung und damit einer anhaltenden und überproportionalen Verteuerung der Rohstoffe entwickelte sich eine Phase des ausreichenden Rohstoffangebotes.

In der ersten Hälfte der achtziger Jahre konnte bei einigen Rohstoffen sogar von einem zyklischen Überangebot gesprochen werden, ${ }^{49}$ eine Tatsache, die sich außer durch einen weltweiten Nachfragerückgang mit einem verstärkten Ausbau der Produktionskapazitäten, besonders in einigen Entwicklungsländern, erklären läßt. Finanzielle Schwierigkeiten, bedingt durch Schuldendienst und Preisverfall, sollten in einigen Produzentenländern (z.B. Brasilien, Chile, Peru) durch einen erhöhten Mengenaussto $B$ kompensiert werden. Die Konsequenz dieser Maßnahmen war ein weiter rückläufiges Preisniveau über die erste Hälfte der achtziger Jahre hinaus. Bei einer Kuppelproduktion, wie sie im Bergbau häufig anzutreffen ist, kann sich ein solches Verhalten auch auf die Märkte anderer Rohstoffe negativ auswirken. ${ }^{50}$

45 Ebenda, S. 8.

46 Vgl. Sames, W.: Welt im Rohstoffüberfluß, in: Metall, 38. Jg., Heft 4, April 1984, S. 325.

47 Vgl. Saager, R.: Metallische Rohstoffe ..., a.a.O., S. 21.

48 Vgl. Hoffmeyer, M.; Schrader, J.V.: Tendenzwandel auf den Rohstoffmärkten, in: Die Weltwirtschaft, Februar 1984, S. 130.

49 Vgl. O.V.: Die merkwürdige Rohstoff-Baisse, in: Frankfurter Allgemeine Zeitung, 10. Oktober 1984.

$50 \mathrm{Vgl}$. Stevens, C.: Commodity prices in the 1980s: a decade of decline for the ACPs, a.a.O., S. 61 f.; Sames, W.: Welt im Rohstoffüberfluß, a.a.O., S. 325. 
Bei einigen Metallen, insbesondere bei Kupfer, sind in den letzten Jahren die Produktionskosten gesunken, teils durch das Erschließen neuer Lagerstätten, teils durch technologischen Fortschritt bei schon bestehenden Abbaubetrieben.

Bedingt durch Interdependenzen zwischen den einzelnen Rohstoffteilmärkten ist für die mineralischen Rohstoffe ein starkes Abweichen von der allgemeinen Preisentwicklung ungewöhnlich.

Trotzdem können auch bei Bergbauerzeugnissen, sofern außergewöhnliche Einflüsse wie Streiks, Veränderung der nationalen Reservebestände oder Spekulationen zum Tragen kommen, Preisdiskontinuitäten eintreten ${ }^{51}$ (s. Abb. 8). Insofern schildern globale Aussagen die Situation nur sehr oberflächlich, da die marktrelevanten Charakteristika, die jedem mineralischen Rohstoff eigen sind, zum Teil erheblich voneinander abweichen können. Daher wird im folgenden auf die im Sysmin-Verfahren explizit erfaßten Bergbauerzeugnisse (außer Uran) näher eingegangen.

\section{Kupfer}

Dieses Buntmetall ist vornehmlich in zwei Lagerstättentypen zu finden, den magmatisch entstandenen Kupferporphyren (z.B. an der Westküste Amerikas und dem ostasiatischen Inselbogen) oder den sekundären Sandsteinvorkommen (z.B. der Kupfergürtel im südlichen Afrika). ${ }^{52}$

Die Entwicklungsländer besaßen im Jahre 1985 einen Anteil von nahezu 60 Prozent an der Weltkupferproduktion, ${ }^{53}$ die USA haben ihre lange Zeit behauptete Vormachtstellung als größter Kupferproduzent verloren. Seit dem Jahre 1982 nimmt Chile vor den USA und der ehemaligen UdSSR diese Position ein, wozu erhebliche Wettbewerbsvorteile ${ }^{54}$ und die

${ }^{51} \mathrm{Vgl}$. Müller-Ohlsen, L.: Die Weltmetallwirtschaft im industriellen Entwicklungsprozeß, Tübingen 1981, S. 188.

52 Vgl. Krüger, J. et al.: Kupfer, in: Gocht, W. (Hrsg.): Handbuch der Metallmärkte, Berlin/Heidelberg/New York 1985, S. $179 \mathrm{ff}$.

${ }^{33}$ Eigene Berechnungen nach Mining Annual Review 1986, S. 27.

${ }^{54}$ O.V.: Chiles Produktionskosten konkurrenzlos günstig, in: Handelsblatt, 30. August 1984. 
Abbildung 8: Die Preisentwicklung der Sysmin-Rohstofe (Basis $1973=100$ )
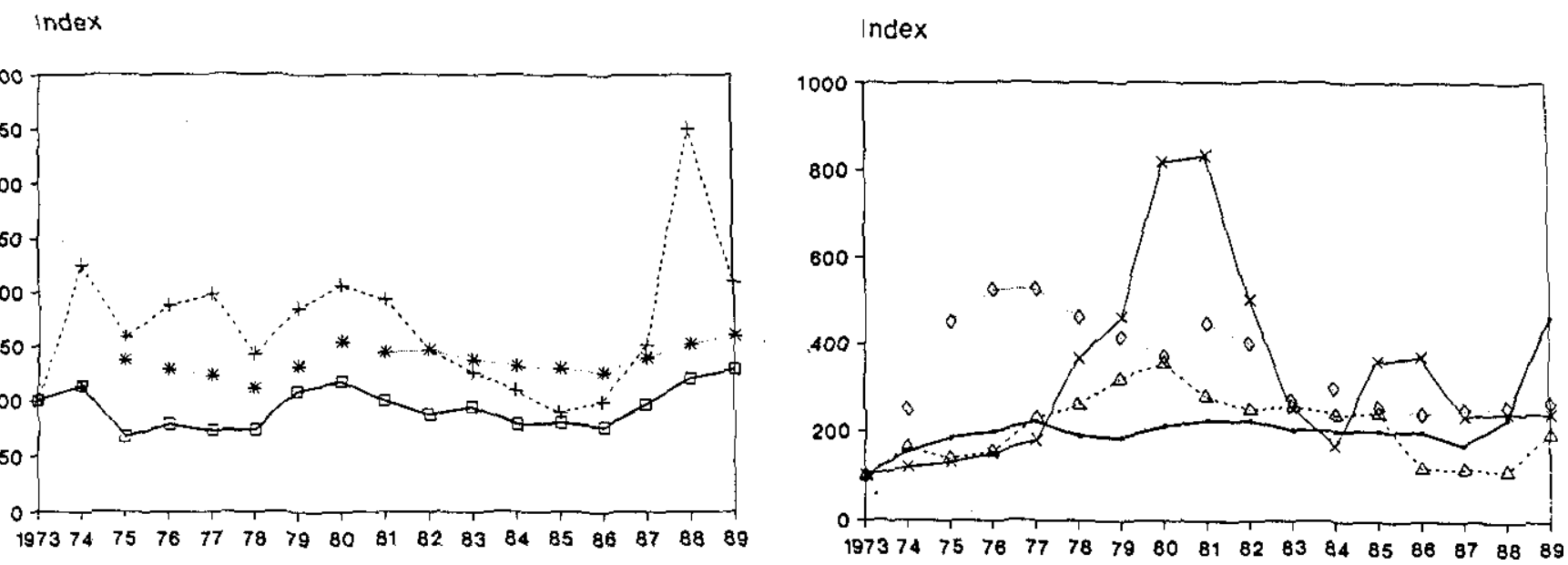

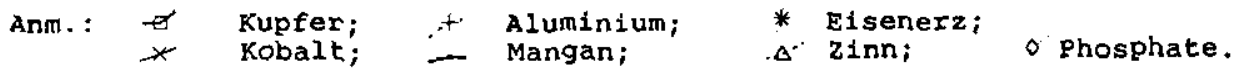

Quelle: Eigene Berechnung nach: Mining Annual Review, Jahrgänge 1976 bis 1990; Metallgesellschaft (Hrsg.): Metallstatistik 1979/1989, Frankfurt/M. 1990. 
Wachstumspolitik der chilenischen Regienung5s beigetragen haben. Die Kupferproduktion ist mit einem Anteil von über 70 Prozent bei 5 Staaten recht stark konzentriert, ${ }^{56}$ auch wenn die Konzentration im Vergleich zu den fünfziger Jahren rückläufig ist. ${ }^{57}$ Die Weiterverarbeitung der geförderten Kupfererze ist in den Produktionsländern - auch in den unterentwickelten - mit über 75 Prozent sehr stark ausgeprägt. ${ }^{58}$

Gehandelt wird Kupfer hauptsächlich als Elektrolytkupfer (Kathoden oder Drahtbarren) mit einem Reinheitsgehalt von 99,7 Prozent bis 99,9 Prozent. Die Preisbildung findet im wesentlichen an der Londoner Metallbörse (LME) und der New Yorker Commodity Exchange (Comex) statt.

Im Gegensatz zu anderen mineralischen Rohstoffen konnte das seit Mitte der siebziger Jahre bestehende Preistief (s. Abb. 9a) erst im letzten Viertel der achtziger Jahre überwunden werden. Eine Betrachtung in Realpreisen (Basis 1980) zeigt, daß der Kupferpreis (Drahtbarren, LME) im Jahre 1986 um nahezu 70 Prozent unter dem Durchschnitt der Periode 1970 1974 lag. Erst ab dem Jahre 1987 war eine Preiserholung festzustellen.59 Dennoch ist wegen zeitweilig noch zunehmender Weltvorräte und einer langfristig stagnierenden Nachfrage nach Primärkupfer - bedingt durch technischen Fortschritt, Substitution und Recycling - in Zukunft nicht mit einer nachhaltigen Änderung des Kupferpreises zu rechnen. Seit Beginn der achtziger Jahre ist bei vielen Kupferproduzenten eine deutliche $\mathbf{R e}$ duktion der Produktionskosten festzustellen. Dies trifft insbesondere für die US-amerikanischen Unternehmen $z \mathbf{u}$, wo die Kosten seit dem Jahre 1981 um ca. ein Drittel gesenkt werden konnten. ${ }^{60}$

55 Vgl. O.V.: Economy struggles as copper stays down, in: Africa Research Bulletin, Oktober - November 1983, S. 7047.

56 Vgl. O.V.: Kupfer: Vorräte reichen langfristig zur Deckung des Verbrauchs, in: Handelsblatt, 5. Juli 1990.

57 Vgl. Schmidt, H.; Kruszona, M.: Regionale Verteilung ..., a.a.O., S. 24.

$58 \mathrm{Vgl}$. Müller-OhIsen, L.: Die Weltmetallwirtschaft ..., a.a.O., S. 122.

$59 \mathrm{Vgl}$. ACP-EEC Assembley Report on Commodities (Hrsg.): Commodity studies, a.a.O., S. 76.

${ }^{60}$ Vgl. O.V.: Copper, Short-term shine, in: The Economist, Mai 1987, S. 69. 
Abbildung 9a-g: Die Produktions- und Preisentwicklung der SysminRohstoffe (1973 - 1989)

a) Kupfer

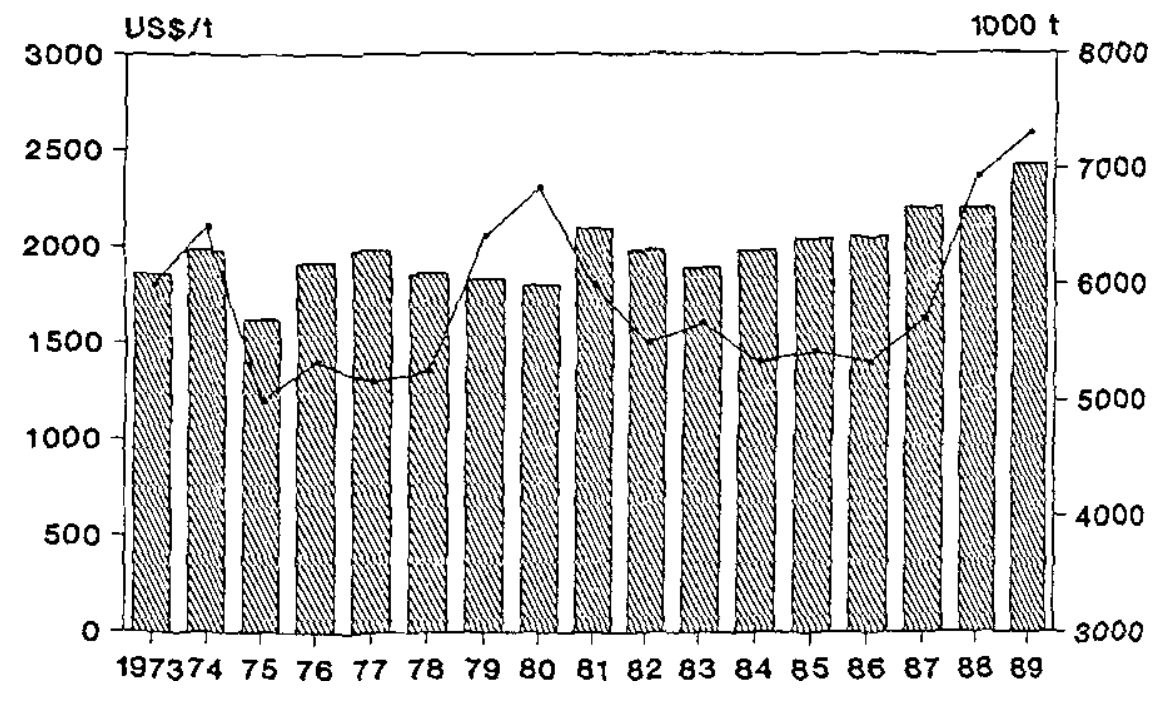

PIeis

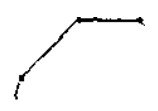

Produktion

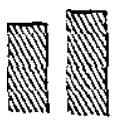

Anm.: Ohne Staatshandelsländer, LME-Preise für Drahtbarren.

Quelle: Eigene Berechnung nach: Mining Annual Review, Jahrgänge 1976 bis 1990; Metallgesellschaft (Hrsg.: Metallstatistik 1979/1989, Frankfurt/M. 1990.

Ein bedeutender Teil des Buntmetalles wird von der Automobil- und Elektroindustrie (ca. 34 Prozent) sowie dem Bausektor (ca. 22 Prozent) verarbeitet (vgl. Anhang 3). Bei der Nachfrage kann somit von einem schwachen Teiloligopol ausgegangen werden. ${ }^{61}$

6! Vgl. Krüger, J., et al.: Kupfer, a.a.O., S. 206. 
$\underline{\text { Kobalt }}$

Kobalt tritt nahezu ausschließlich in der Verbindung mit Kupfer- oder Nickelvorkommen auf und wird daher vorwiegend als Kuppelprodukt gewonnen.

Die Kobaltvorräte befinden sich zu 63 Prozent in Entwicklungsländern, in den Industrieländern liegt der Anteil bei 10 Prozent. ${ }^{62}$ Mit diesen Verteilungsstrukturen weist Kobalt bei den Stahlveredlern das für die Industrieländer ungünstigere Verhältnis auf.

Die größten Kobaltproduzenten sind Zaire und Sambia mit einer Förderung von $10.677 \mathrm{t}$ und 4.290 $\mathrm{t}$ Metallinhalt im Jahre $1985 . .^{63}$ Diese beiden Staaten Zentralafrikas produzieren somit über 60 Prozent des nicht in Staatshandelsländern gewonnenen Kobalts.

Diese Konzentration war bis vor wenigen Jahren noch ausgeprägter; lange Jahre hatte allein Zaire über 50 Prozent des nicht in Staatshandelsländern geförderten Kobalts produziert. ${ }^{64}$

In den letzten Jahren jedoch ist ein Rückgang der Konzentration auf dem Kobaltmarkt zu erkennen, was nicht nur mit der Abnahme der zairischen Kupferproduktion um nahezu 50 Prozent zu erklären ist, ${ }^{65}$ sondern auch mit der Erschließung weiterer kobaltführender Lagerstätten. ${ }^{66}$ Dazu zählen auch Kobaltkrusten auf dem Meeresboden vor Hawaii, mit deren Abbau in naher Zukunft begonnen werden könnte.

Die oligopolistische Anbieterstruktur der Kobaltproduzenten erklärt den starken Preisanstieg in den Jahren 1977 bis Anfang 1979 (s. Abb. 9b). Angolanische Rebellen erzwangen in der zairischen Provinz Shaba nahezu die Einstellung der Kobaltproduktion. Dies löste Spekulationskäufe aus.

62 Vgl. Kürsten, M: Geographische Verteilung ..., a.a.O., S. 5.

${ }^{63}$ Vgl. Mining Annual Review 1986, S. 71.

${ }^{64} \mathrm{Vgl}$. O.V.: Cobalt, back from the brink, in: Mining journal, Vol. 300, Nr. 7707, 6. Mai 1983, S. 301.

65 Vgl. Schmidt, H.; Kruszona, M: Regionale Verteilung ..., a.a.O., S. 23; 0.V.: Cobalt: back ..., a.a.O., S. 301.

66 Vgl. Saager, R.: Metallische Rohstoffe ..., a.a.O., S. 137. 
Abbildung 9: Die Produktions- und Preisentwicklung der Sysmin-Rohstoffe (1973 - 1989)

b) Kobalt

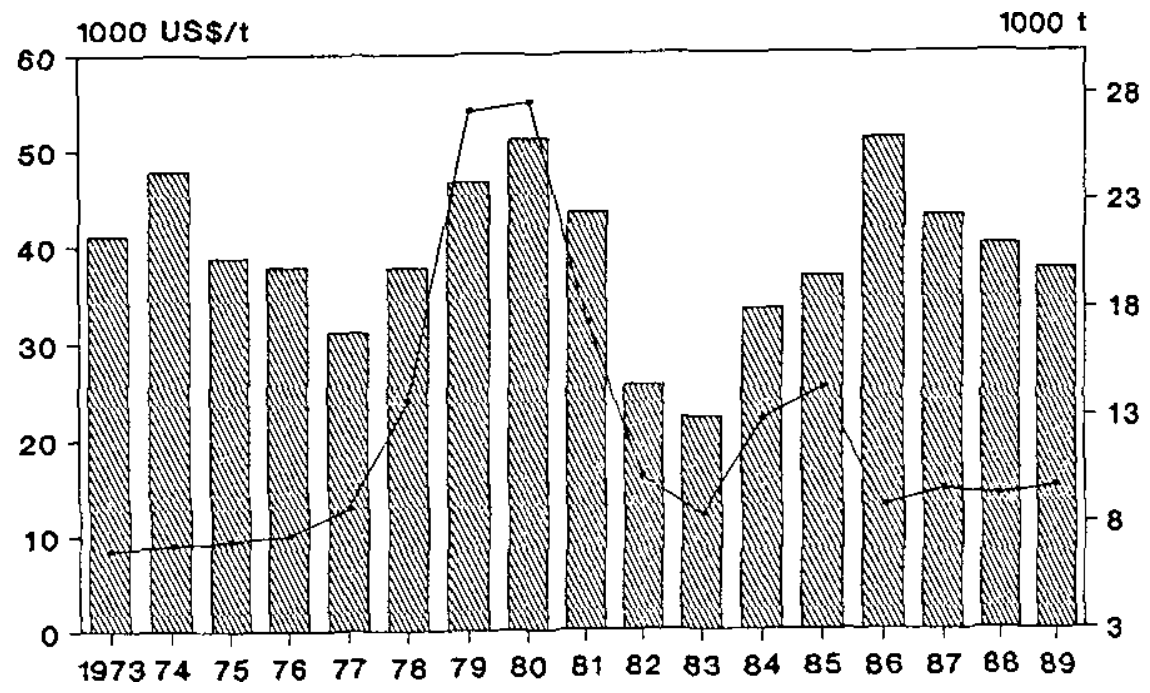

Anm.: Ohne Staatshandelsländer, Preise für $99,5 \%$ reines Kobalt.

Quelle: s. Abbildung 9 a

Bei den Preisen für Kobalt handelt es sich um Produzentenpreise. Aufgrund der Vormachtstellung der zairischen Bergbaugesellschaft Gecamines wird der Kobaltpreis in der Regel durch die zairische Handelsorganisation Sozacom festgelegt. Die Ermittlung des Preisniveaus an der Londoner Metallbörse (LME) spielt nur eine untergeordnete Rolle. ${ }^{67}$

67 Vgl. Gécamines (Hrsg.): Rapport Annuel 1981, Kinshasa (1982), S. 21; Gocht, W. (Hrsg.): Handbuch der Metallmärkte, Berlin/Heidelberg/New York 1985, S. 129. 
Anfang der achtziger Jahre verursachte der rezessiv bedingte Nachfragerückgang - verbunden mit einem ruinösen Wettbewerb und Vorabverkäufen - bei Kobalt einen, im Vergleich zu anderen Metallen, besonders starken Preisverfall. ${ }^{68} \mathrm{Im}$ Jahre 1985 war wieder eine Produktionszunahme um etwa 40 Prozent gegenüber dem Jahre 1983 zu verzeichnen. ${ }^{69}$

Kobalt wird auf dem Weltmarkt hauptsächlich als Elektrolytkobalt oder Elektrolytgranulat gehandelt, ${ }^{70}$ meistens bestehen langfristige Lieferabkommen zwischen den Erzeugern und den Abnehmern.

Verwendung findet Kobalt als Legierung (überwiegend für hoch beanspruchte Teile wie Industrieöfen, Turbinenschaufeln) oder als chemische Verbindung (Farben, Katalysatoren). Die Hauptabnehmer setzen sich in der Europäischen Gemeinschaft wie folgt zusammen: Eisen- und Stahlindustrie (ca. 19 Prozent), Elektroindustrie (ca. 18 Prozent), Luft- und Raumfahrtindustrie (ca. 14 Prozent) ${ }^{71}$.

\section{Phosphate}

Als einziges nichtmetallisches Bergbauprodukt werden Phosphate in dem Sysmin-Katalog erfaßt (s. Kap. 2.5.3).

Ähnlich wie bei Kobalt sind die Phosphatvorräte aus Sicht der Industriestaaten ungünstig verteilt. Während in diesen Ländern nur 17 Prozent der Weltvorräte anzutreffen sind, befinden sich in den Entwicklungsländern ca. 70 Prozent. ${ }^{72}$ Jedoch besitzen die Phosphate keine strategische Bedeutung. So tritt bei der Hauptverwendung als Düngemittel Stickstoffoder Kalidünger als Substitutionskonkurrenz auf, außerdem läßt sich Phosphorsäure auch als Abfallprodukt bei der Stahlerzeugung (Thomasschlacke) gewinnen. ${ }^{73}$

${ }^{68}$ Vgl. Mining Annual Review 1984, S. 13.

69 Vgl. Mining Annual Review 1986, S. 71.

${ }^{70}$ Vgl. O.V.: Cobalt: back ..., a.a.O., S. 301; Mining Annual Review 1984, S. 69.

7 Vgl. Gocht, W. (Hrsg.): Handbuch der Metallmärkte, a.a.O., S. $121 \mathrm{f}$.

72 Vgl. Kürsten, M.: Geographische Verteilung ..., a.a.O., S. 7.

${ }^{73}$ Vgl. Tietzel, M.: Internationale Rohstoffpolitik, Bonn 1977, S. 68 f. 
Die Konzentration bei der Phosphatproduktion hat sich abgeschwächt, ${ }^{74}$ obwohl auch im Jahre 1985 in den USA, der ehemaligen UdSSR, Marokko und China über 75 Prozent der Weltproduktion gefördert wurde.

Auch der Phosphatabsatz hatte unter der rezessiven Entwicklung der Weltwirtschaft in der ersten Hälfte der achtziger Jahre zu leiden (s. Abb. 9c).

Obwohl der Handel mit diesem Rohstoff seit dem Jahre 1983 wieder 2unimmt, erscheint der Markt nicht in der Lage zu sein, den Produktionsüberschuß zu absorbieren. ${ }^{75}$

Als Folge der Überkapazitäten (1983: 25 Prozent) und des Auftretens weiterer Phosphatanbieter ist - deflationiert - ein Preisverfall zu verzeichnen. Freiwillige Kapazitätsbeschränkungen, vornehmlich in den USA, versuchen diesem Trend entgegenzuwirken. ${ }^{76}$ Die mögliche Gewinnung von Phosphatknollen auf dem Meeresboden würde das Überangebot weiterhin vergrößern.

Überwiegend finden Phosphate in der Agrarindustrie als Dünger Verwendung (ca. 76 Prozent; Auf den Einsatz von Phosphaten bei der Waschmittelherstellung ist aufgrund der Umweltrelevanz seit jüngster Zeit nahezu völlig verzichtet worden. Vgl. Anhang 3).

\section{Aluminium}

Die wichtigsten Ausgangsstoffe für das in der Natur nicht elementar auftretende Aluminjum sind die Verwitterungserze Bauxit und Kaolin.

Die Lagerstätten der gegenwärtig gewinnbaren Bauxitvorräte sind nahezu ausschließlich in subtropischen Regionen anzutreffen, wobei es sich vor-

74 Vgl. Schmidt, H.; Kruszona, M.: Regionale Verteilung ..., a.a.O., S. 23.

$75 \mathrm{Vgl}$. Mining Annual Review 1986, S. 101; Mining Annual Review 1984, S. 98.

76 Vgl. Mining Annual Review 1984, S. 98 ff. 
Abbildung 9: Die Produktions- und Preisentwicklung der Sysmin-Rohstoffe (1973 - 1989)

c) Phosphate

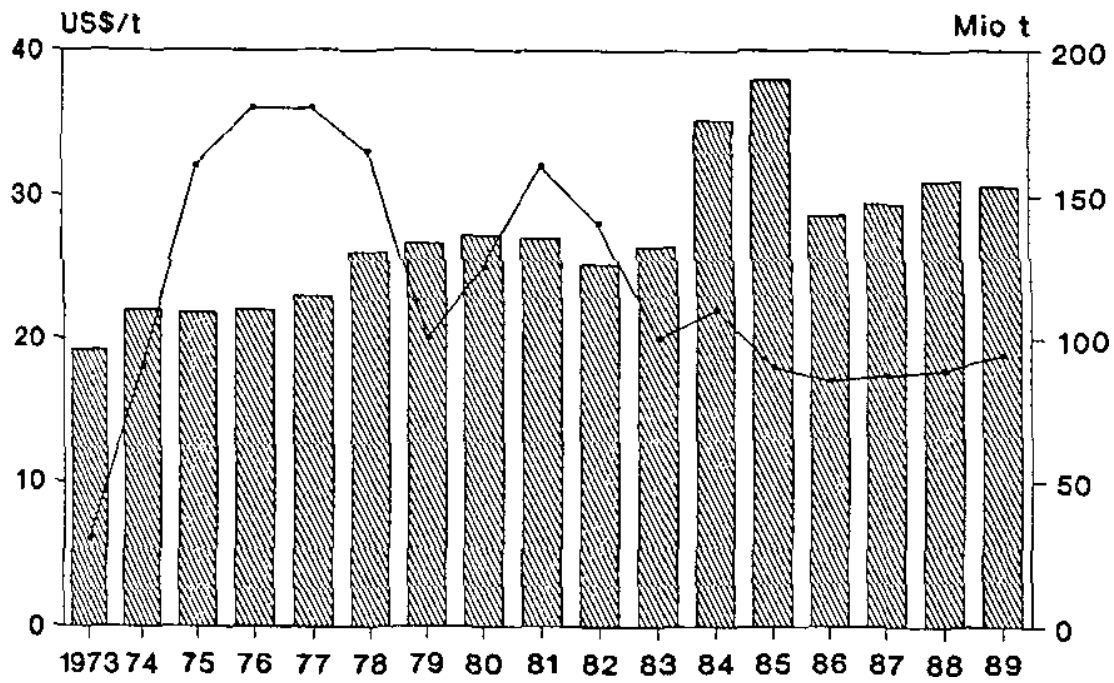

Anm: Preise für Phosphate mit 66-68 BPL.

Quelle: s. Abbildung 9 a

wiegend um - nach dem Ausgangsgestein bezeichnete - Silikatbauxite handelt. ${ }^{77}$

Die bedeutendsten Bauxitproduzenten sind in Australien, Afrika und Mittelamerika anzutreffen. So erklärt es sich, daß die Staaten mit der größten Aluminiumproduktion USA und Kanada, aber auch die ehemalige UdSSR, um ihren Bedarf zu decken, im wesentlichen auf Importe zurückgreifen müssen. ${ }^{78}$

77 Vgl. Herda, M.: Leichtmetalle, in: Gocht, W. (Hrsg.): Handbuch der Metallmärkte, Berlin/Heidelberg/New York 1985, S. $320 \mathrm{ff}$.

$78 \mathrm{Vgl}$. Saager, R.: Metallische Rohstoffe ..., a.a.O., S. 140. 
Die Bauxitproduktion außerhalb der Staatshandelsländer ist mit über 75 Prozent auf Australien, Guinea, Brasilien und Jamaika konzentriert. Generell ist eine Konzentrationszunahme zu verzeichnen, da aufgrund der bestehenden Überkapazitäten in der Bauxit- und Aluminiumproduktion in den letzten 10 Jahren außer in Australien keine neuen Großtagebaue erschlossen wurden. ${ }^{79}$

Der erhebliche Energiebedarf bei der Aluminiumerzeugung erklärt die häufig anzutreffende Trennung zwischen Bauxitgewinnung, Tonerdeproduktion und Aluminiumerzeugung. Bedingt durch die steigenden Energiekosten etablierten sich in der ersten Hälfte der achtziger Jahre mehr und mehr Rohmetallproduzenten an Orten mit größeren Elektrizitätskapazitäten (Wasserkraft bzw. Erdgas als Kuppelprodukt bei der Erdölförderung). Das bedeutete für die sechs großen integrierten Aluminiumhersteller einen zunehmenden Verlust ihres Markteinflusses ${ }^{80}$ Gehandelt wird Aluminium in Rein- und Reinstform (Barren mit 99,0 oder 99,9 Prozent) und als Legierung. Seit Ende der siebziger Jahre wird Aluminium auch an der Londoner Metallbörse (LME) und seit Dezember 1983 auch an der New York Commodity Exchange (Comex) gehandelt, eine Tatsache, die den oben erwähnten Trend des sinkenden Einflusses der weltgrößten Aluminiumproduzenten verstärkt. Gleichwohl gibt es für Bauxit oder Tonerde noch keinen eigentlichen Markt, da sich immer noch sowohl die Lagerstätten als auch die Hüttenwerke hauptsächlich in den Händen der großen Aluminiumhersteller befinden. ${ }^{81}$

Die Belebung der Aluminiumnachfrage seit dem Jahre 1983 (s. Abb. 9d) sowie freiwillige Produktionseinschränkungen bewirkten im Jahre 1985 ein geringes Angebotsdefizit. Neubauten und die Erweiterung bzw. Modernisierung von Hütten, unter anderem in Australien, Kanada und Brasilien, lassen für die neunziger Jahre wieder ein Überangebot an Hüttenaluminium erwarten. ${ }^{82}$ Das verstärkte Auftreten der kostengünstig pro-

79 Vgl. Schmidt, H.; Kruszona, M.: Regionale Verteilung ..., a.a.0., S. 23; Harms, U.; Iwersen, A.: Rohstoffgewinnung in Entwicklungsländern, ite, Hamburg Oktober 1981, S. 21; Mining ADnual Review 1987, S. 41 und S. 43 f.

$80 \mathrm{Vgl}$. Spreng, D.T.: Tiefgreifender Strukturwandel in der weltweiten Aluminiumindustrie, in: Neue Zürcher Zeitung, 1. August 1981.

$81 \mathrm{Vgl}$. Herda, M.: Leichtmetalle, a.a.O., S. $340 \mathrm{f}$.

82 Vgl. Metallgesellschaft (Hrsg.): Metallstatistik 1975 - 1985, Frankfurt/M. 1986, S. VI, Mining Annual Review 1987, S. 41. 
duzierenden Unternehmen in Indonesien und Brasilien, die in der zweiten Hälfte der achtziger Jahre durchgeführten Kostenreduktionen und der zunehmende Einsatz von Recycling-Material und Substituten lassen einen nachhaltigen Preisanstieg unwahrscheinlich erscheinen. ${ }^{83}$ Der Bauxitpreis, die AKP-Staaten exportieren im wesentlichen Bauxit, konnte vom Anstieg der Aluminiumpreise nicht profitieren. Im Jahre 1987 betrug der US-Import Reference Price für Jamaika (Realpreis, Basis 1980) nur noch etwa 50 Prozent des Durchschnittspreises aus der zweiten Hälfte der siebziger Jahre. ${ }^{84}$

Über 20 Prozent des Aluminiums wird im Fahrzeugbau (PKW, Schienenund Luftfahrzeuge) eingesetzt, ca. 16 Prozent im Bausektor. Eine zunehmende Verwendung (zur Zeit ca. 8 Prozent) erfährt Aluminium in der Verpackungsindustrie (vgl. Anhang 3). ${ }^{85}$

\section{Mangan}

Nach Eisen ist heutzutage Mangan das mengenmäßig am häufigsten erzeugte Metall. ${ }^{86}$ Etwa 53 Prozent der Vorräte befinden sich in den westlich orientierten Bergbauländern - davon 45 Prozent in der Republik Südafrika und 7 Prozent in Australien. Die Entwicklungsländer vereinigen 9 Prozent der bekannten Vorräte auf sich, davon 5 Prozent in Gabun und 2 Prozent in Brasilien. ${ }^{87}$

Die Manganerzproduktion stammt gegenwärtig hauptsächlich aus marinsedimentären oder metamorphen, d.h. sedimentär gebildeten Lagerstätten, die in den tropischen Regionen durch lateritische Verwitterung starke Anreichungen erfahren haben. ${ }^{88}$ Die erheblichen Mengen von sogenannten

83 Vgl. Hoffmeyer, M.; Schrader, J.V.: Tendenzwandel auf den Rohstoffmärkten, a.a.O., S. 132; O.V.: Aluminium, in: Financial Times, Section IV, 26. Oktober 1988, S. II.

$84 \mathrm{Vgl}$. ACP-EEC Assembley Report on Commodities (Hrsg.): Commodity studies, a.a.O., S. 76.

85 Pro PKW werden durchschnittlich $45 \mathrm{~kg}$ Aluminium verarbeitet. Vgl. 0.V.: Bedeutendster Abnehmer ist der Fahrzeugbau, in: Handelsblatt, 11. August 1986.

${ }^{86} \mathrm{Vgl}$. Schmidt, H.; Kruszona, M.: Regionale Verteilung .., a.a.O., S. 8.

87 Vgl. Kürsten, M.: Geographische Verteilung ..., a.a.O., S. 5.

$88 \mathrm{Vgl}$. Gocht, W. (Hrsg.): Handbuch der Metallmärkte, a.a.O., S. 73 f. 
Abbildung 9: Die Produktions- und Preisentwicklung der SysminRohstoffe (1973 - 1989)

d) Aluminium

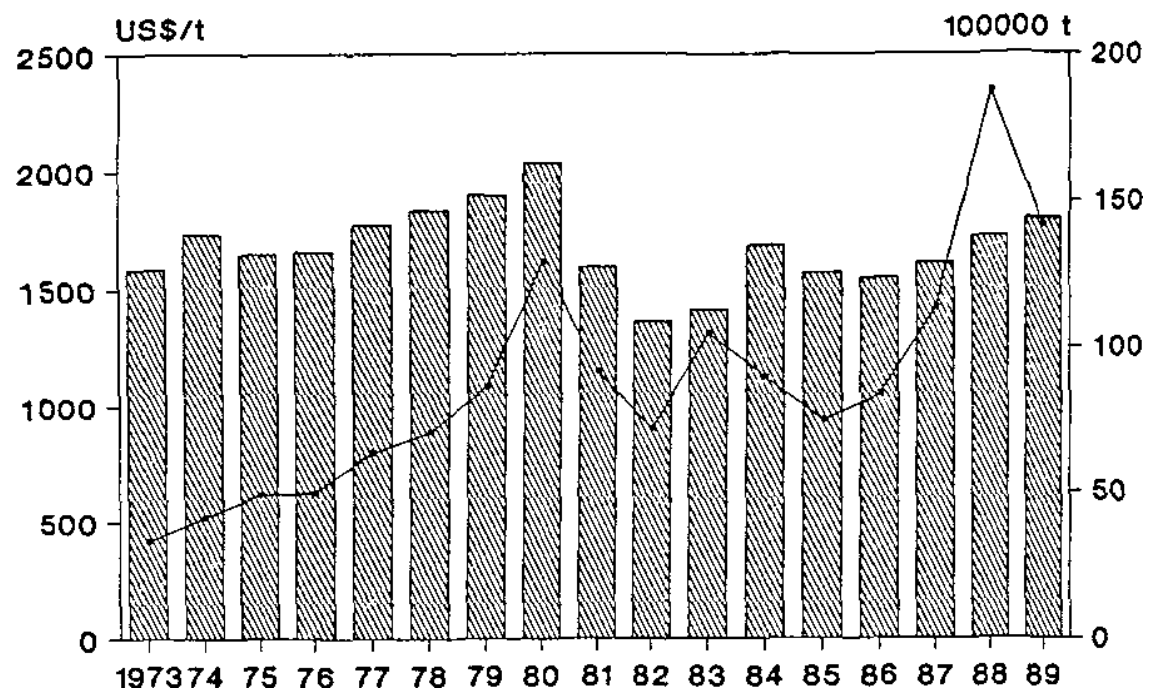

Anm.: Preise für 99,5\% reines Aluminium.

Quelle: s. Abbildung 9 a

Manganknollen auf den Tiefseeböden werden zur Zeit noch nicht abgebaut.

Obwohl bei Mangan nie die Gefahr einer Unterversorgung aufgrund unzureichender Vorräte bestand, und über 40 Prozent der Erzproduktion (ohne Staatshandelsländer) aus Industrieländern stammt, zählt Mangan neben Chrom und Vanadium zu den am stärksten durch politische Versorgungsstörungen (Südafrika) gefährdeten Rohstoffen ${ }^{89}$ (s. Kap. 3.1).

Der Markt für Manganerz ist eng mit dem Eisenerzmarkt verknüpft. Obwohl die ehemalige UdSSR als größter Manganproduzent mit einer baldi-

$89 \mathrm{Vgl}$. Bundesministerium für Wirtschaft (Hrsg.): Mineralische Rohstoffe - Märkte und Perspektiven, a.a.O., S. 80. 
gen Erschöpfung ihrer reichsten Lagerstätte zu rechnen hat, ${ }^{90}$ können Preiseinbrüche wie im Jahre 1983 (10 - 15 Prozent unter dem Vorjahresniveau, s. Abb. 9e) nur durch zusätzliche Produktionseinschränkungen vermieden werden. ${ }^{91}$

Mangan wird ähnlich wie Kobalt als Legierungsmetall verwendet (ca. 90 Prozent im Fahrzeug-, Maschinen- und Anlagenbau). In der chemischen

Abbildung 9: Die Produktions- und Preisentwicklung der Sysmin-Rohstoffe (1973 - 1989)

e) Mangan

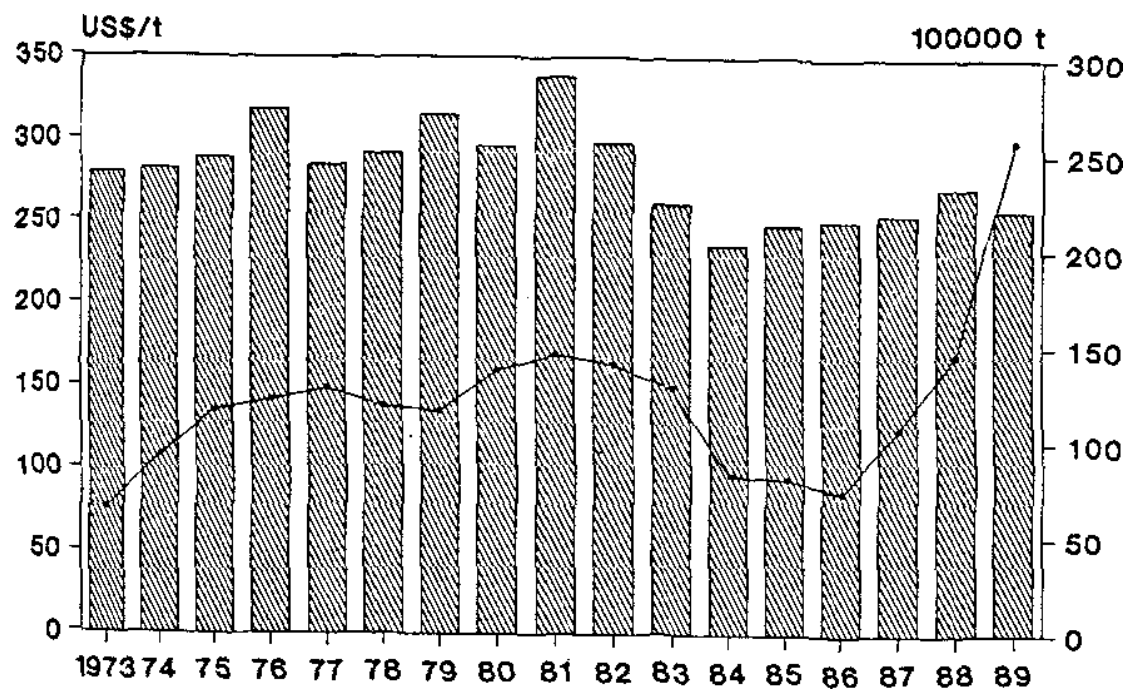

Anm.: Produktion geschätzt, Preise für Manganerz mit mindestens $40 \%$ Metallinhalt.

Quelle: s. Abbildung 9 a

${ }^{90}$ Vgl. Saager, R.: Metallische Rohstoffe ..., a.a.O., S. 110.

91 Vgl. Mining Annual Review 1984, S. 65. 
Industrie werden etwa 5 Prozent des Mangans verbraucht (Düngemittelzusatz, Batterieherstellung, Katalysator) ${ }^{92}$ (vgl. Anhang 3).

\section{$\underline{\text { Zinn }}$}

Neben den primären - meist gangförmigen - Zinnlagerstätten (z.B. Bolivien, Australien) wird das wirtschaftlich bedeutendste Zinnmineral, der Kassiterit, vornehmlich aus sekundären Lagerstätten, den Zinnseifen, gewonnen (z.B. Malaysia). ${ }^{93}$

Ähnlich wie bei Kobalt herrscht auch bei den Zinnvorräten eine für die Industrieländer ungünstige Verteilung. Dort sind lediglich 8 Prozent der Weltvorräte dieses NE-Metalles anzutreffen. Dagegen befinden sich 66 Prozent der Vorräte in den Entwicklungsländern, wobei die asiatischen Staaten Indonesien, Thailand und Malaysia zusammen 40 Prozent der Weltvorräte an Zinn besitzen. ${ }^{94}$

Die 3 asiatischen Zinnländer produzierten 1985 gemeinsam mit Bolivien über 60 Prozent der Zinnkonzentrate. Durch das Auftreten weiterer Zinnproduzenten am Weltmarkt hat seit Beginn der achtziger Jahren die Konzentration bei den Zinnanbietern abgenommen. Brasilien beispielsweise steigert seinen Marktanteil kontinuierlich (seit 1984 höhere Produktion als Bolivien; 1982 - 1985 Verdreifachung der Konzentratproduktion) und fühlte sich, da es nicht dem Internationalen Zinnabkommen (ITA) angehörte, auch nicht an die ab dem Jahre 1982 festgesetzten Exportbeschränkungen gebunden. ${ }^{95}$

Die deutliche Steigerung der Zinnproduktion, besonders in den NichtITA-Ländern Bolivien, China und Brasilien sowie ein starker Nachfragerückgang, zum Teil auch durch die erfolgreiche Preispolitik des Internationalen Zinnrates bedingt (Substitution), sorgte seit dem Jahre 1982 fur ein Überangebot. Durch eine vom Internationalen Zinnrat verfuigte und im Jahre 1983 verschärfte Exportkontingierung und durch Maßnahmen der Bufferstockmanager (Verdoppelung der Einlagermenge) konnte vorerst ein allzu heftiger Abfall des Zinnpreises vermieden werden (s. Abb. 9f). Ende

92 Vgl. Gocht, W. (Hrsg.): Handbuch der Metallmärkte, a.a.O., S. 81 f.

93 Ebenda, S. 259 f.

94 Vgl. Kürsten, M., Geographische Verteilung ..., a.a.O., S. 6.

95 Vgl. Mining Annual Review 1986, S. 38. 
Oktober 1985 jedoch mußte der Zinnhandel an der Londoner Metallbörse (LME) ausgesetzt werden, da den Bufferstockverwaltern keine weiteren Mittel zur Verfügung gestellt wurden, um zusätzliche Stützungskäufe zu tätigen. ${ }^{96}$ Daraufhin ist der Zinnpreis in kurzer Zeit um über 50 Prozent zurückgegangen (Okt. 1985: $8.140 \mathrm{GBP} / \mathrm{t}$; März 1986: $3.400 \mathrm{GBP} / \mathrm{t}$ ). ${ }^{97}$

Abbildung 9: Die Produktions- und Preisentwicklung der Sysmin-Rohstoffe (1973 - 1989)

f) Zinn

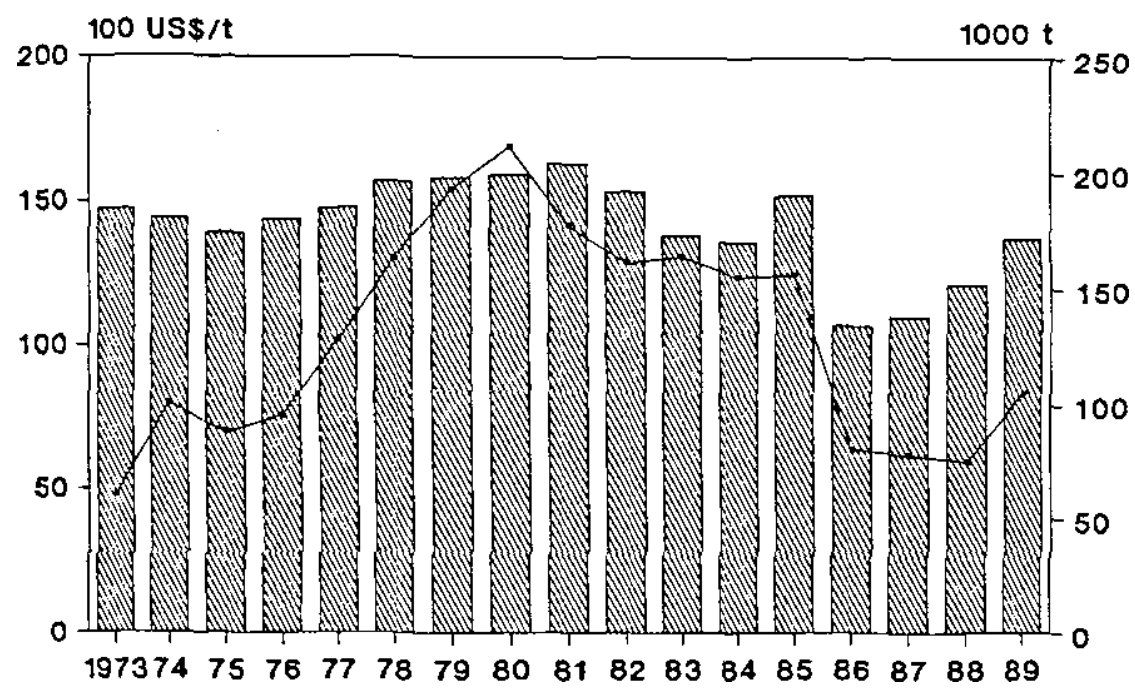

Anm.: Ohne Staatshandelsländer, LME-Preise für 99,75\% reines Zinn. Zwischen Oktober 1985 und Juli 1989 kein Zinn-Handel an LME.

Quelle: s. Abbildung 9 a

${ }^{96}$ Vgl. Krause, K.P.: Die unbewältigte Zinnkrise, in: Frankfurter Allgemeine Zeitung, 10. Februar 1986.

97 Vgl. Wilkinson, M.: Tin price climbs back above GBP 4.000 a ton, in:

Financial Times, 22. Oktober 1986. 
Nachdem sich Marginalproduzenten - besonders in Südostasien - verstärkt gezwungen sahen, die Zinnproduktion einzustellen (z.B. Zinnminen in Malaysia 1984: 480; 1985: 280; Okt. 1986: 187), ist seit der zweiten Hälfte 1986 wieder eine leichte Erholung der Zinnpreise festzustellen (März 1989: $4.900 \mathrm{GBP} / \mathrm{t}$ ). ${ }^{98}$ Eine weitere Erholung des Zinnpreises ist nur zu erwarten, wenn es der Vereinigung der zinnproduzierenden Länder (ATPC), die sich nach dem Zusammenbruch des Zinnabkommens new formiert hat, weiterhin gelingt, die Exporte zu drosseln.99

Die größten Handelsplätze für Zinn (über 60 Prozent des Handelsvolumens) sind die Londoner Metallbörse und die Kuala Lumpur Commodity Exchange (KLCE). Der Zinnhandel an der New York Commodity Exchange und der Zinnbörse in Penang wurden bereits vor der Zinnkrise eingestellt. Gehandelt wird Zinn als Rein- und Reinstzinn in Barrenform, Stangen oder Blöcken, beziehungsweise als Zinnkonzentrat in verschiedenen Anreicherungsgraden. Zinn wird heutzutage vielfältig eingesetzt, z.B. in Legierungen und Chemikalien, für Verzinnungen. Mit ca. 36 Prozent ist die Eisen- und Stahlindustrie der größte Zinnverwender (s. Abhang 3). ${ }^{100}$

\section{$\underline{\text { Eisenerz }}$}

Eisenerz ist nicht, wie andere mineralische Rohstoffe, auf bestimmte $\mathrm{ZO}^{-}$ nen mit einem gewissen Geocharakter beschränkt, sondern ist in sämtlichen Erdteilen mit scheinbar unerschöpflichen Vorkommen anzutreffen.

Von den verschiedenen Lagerstättentypen sind die marin-sedimentär entstandenen Eisenerzanreicherungen die wirtschaftlich interessantesten. Magmatische und hydrothermale Lagerstätten besitzen häufig nur lokale Bedeutung. ${ }^{101}$

98 Sulong, W.: Tin gloom lifts in Malaysia, in: Financial Times, 31. Oktober 1986.

99 ACP-EEC-Assembley Report on Commodities (Hrsg.): Commodity studies, a.a.O., S. $76 \mathrm{f}$.

${ }^{100} \mathrm{Vgl}$. Gocht, W. (Hrsg.): Handbuch der Metallmärkte, a.a.O., S. 285 f. 101 Vgl. Walther, H.W., et al.: Eisen, in: Gocht, W. (Hrsg.): Handbuch der Metallmärkte, a.a.O., S. $31 \mathrm{ff}$. 
Anfang der achtziger Jahre besaßen die Industrieländer 35 Prozent der Weltvorräte, gegenüber 31 Prozent in den Entwicklungsländern und 34 Prozent in den Staatshandelsländern.

Bei der Eisenerzproduktion ist seit Ende der siebziger Jahre eine Konzentrationsabnahme festzustellen. ${ }^{102}$ Im Jahre 1950 bestritt die Ländergruppe USA, EG-Länder (ohne Spanien und Portugal), die ehemalige UdSSR und Schweden noch 84 Prozent der Weltroherzförderung. Im Jahre 1985 reduzierte sich dieser Anteil auf unter 40 Prozent. Dabei ist jedoch zu berücksichtigen, daß sich die Gesamtmenge des geförderten Erzes in dem betachteten Zeitraum verdreifachte. ${ }^{103}$ Die Bedeutung der Entwicklungsländer bei der Eisenerzproduktion hat immer weiter zugenommen. Mitte der achtziger Jahre gehörten die ehemalige UdSSR, China, Brasilien, Australien und die USA zu den größten Eisenerzerzeugern. ${ }^{104}$

Gehandelt wird Eisen in der Regel als oxidisches Erz (bis zu 65 Prozent Eisenanteil) oder als Pellets (bis zu 60 Prozent Eisenanteil). Der Handel zwischen den Erzproduzenten und der weiterverarbeitenden Industrie ist durch meist langfristige Liefer- und Abnahmekontrakte gekennzeichnet. ${ }^{105}$

Anfang der achtziger Jahre ist der Eisenerzmarkt durch die rezessive Wirtschaftslage stark getroffen worden. Der seit 1950 ständig steigende Verbrauch fing ab Mitte der siebziger Jahre an zu stagnieren, um dann ab dem Jahre 1980 zurückzugehen.

Seit dem Jahre 1984 ist wieder ein Produktionsanstieg festzustellen (s. Abb. 4g); trotzdem waren die Kapazitäten Mitte der achtziger Jahre nur zu etwa $50 \%$ ausgelastet. ${ }^{106}$

Eine Real-Preisbetrachtung (Basis 1980) zeigt, daß der Preis für Eisenerz (cif, Nordseehäfen) im Jahre 1987 einen absoluten Tiefstand mit mehr als 50 Prozent unter dem Preisniveau der Periode 1970 - 1974 erreichte. Pro-

${ }^{102}$ Vgl. Schmidt, H.; Kruszona, M.: Regionale Verteilung ..., a.a.O., S. 40.

${ }^{103}$ Eigene Berechnungen, Vgl. Mining Annual Review 1986, S. 55; Walther, H.W, et al.: Eisen, a.a.O., S. $42 \mathrm{f}$.

${ }^{104} \mathrm{Vgl}$. Mining Annual Review 1986, S. 55.

105 Vgl. Saager, R.: Metallische Rohstoffe ..., a.a.O., S. 104.

106 Vgl. Mining Annual Review 1986, S. 55. 
gnosen für die neunziger Jahre gehen von keiner wesentlichen Änderung des Preisniveaus aus. ${ }^{107}$

Abbildung 9: Die Produktions- und Preisentwicklung der Sysmin-Robstoffe (1973 - 1989)

g) Eisenerz

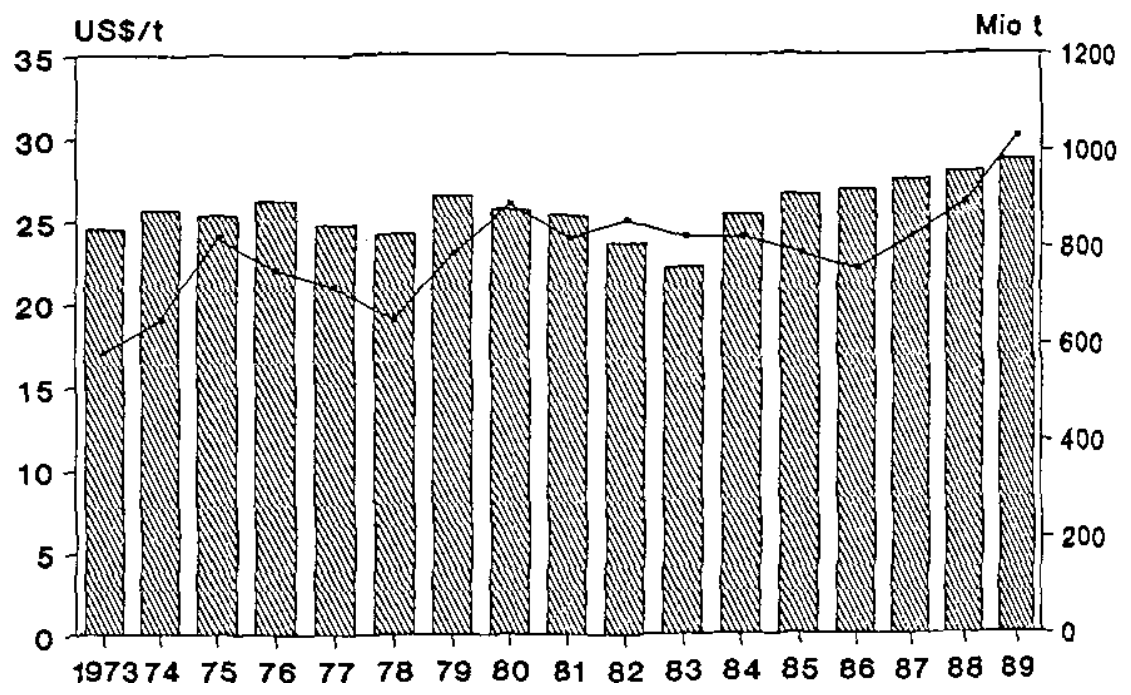

Anm.: Preise für brasilianisches Eisenerz in Nordseehäfen mit mindestens 60 Prozent Metallinhalt.

Quelle: s. Abbildung 9 a

Die Verwendung von Eisen ist vielfältig, etwa 90 Prozent werden zu Stah] verarbeitet.

107 Vgl. ACP-EEC Assembley Report on Commodities (Hrsg.): Commodity studies, a.a.O., S. 76. 
Die bedeutendsten Verwendungsbereiche sind das Bauwesen (ca. 16 Prozent), der Transportsektor (ca. 15 Prozent) und der Maschinenbau (ca. 10 Prozent) (s. Anhang 3).

Generell ist eine Abnahme des Pro-Kopf-Stahlverbrauches aufgrund des techsischen Fortschrittes zu erwarten; der geringer werdende Einsatz von Stahl im Automobilbau (1976: $650 \mathrm{~kg}$ /europ. PKW; 1986: ca. 567 $\mathrm{kg} /$ eutop. PKW) mag dafuir symptomatisch sein. ${ }^{108}$

${ }^{108} \mathrm{Vgl}$. Walther, H.W., et al.: Eisen, a.a.O., S. $66 \mathrm{f}$. 



\section{DIE STÄRKEN UND SCHWÄCHEN DER SYSMIN-REGE- LUNG}

\subsection{Die Durchführbarkeitsbestimmungen der Sysmin-Regelung}

Wie bereits im Kapitel 2.5.3 ausführlich dargelegt, werden in Artikel 215 des Lomé-IV-Abkommens die Konditionen der Sysmin-Fazilität definiert. Im Gegensatz zur Sysmin-Regelung in Lomé II und Lomé III werden nunmehr die Abhängigkeits- und Auslöseschwellen in einem Artikel gemeinsam aufgeführt, wodurch die Transparenz erschwert wird. Angesichts der Aufnahme weiterer Restriktionen sowie durch nicht immer eindeutige Formulierungen ist es nicht gelungen, die Möglichkeiten von Fehlinterpretationen, sie bestanden auch in den Sysmin-Artikeln der Vorgängerabkommen, auszuschließen.

Besonders augenscheinlich sind darüber hinaus auch Abweichungen in den Übersetzungen (hier: englische und deutsche Version), obwohl jede Übersetzung gleichermaßen verbindlich ist (Lomé IV: Art. 369).

Zunächst wird im folgenden näher auf einen Passus eingegangen, der bereits in der Lomé-III-Version nicht eindeutig war und dessen Formulierungen in Lomé IV nun noch zu weiterer Interpretation Anlaß gibt:

Gemäß dem Wortlaut dieser Klausel:1 "Die ... besondere Finanzierungsfazilität richtet sich an die AKP-Staaten, deren Ausfuhren in die Gemeinschaft gehen ...", können nur allein nach der Europäischen Gemeinschaft exportierende AKP-Staaten Anträge auf Sysmin-Mittel stellen. Aufgrund der oben erwähnten Zusammenfassung der Abhängigkeits- und Auslöseschwellen ist nunmehr nicht mehr ersichtlich, ob diese Restriktion nur für die Auslöseschwelle (wie in Lomé III und Lomé II) Gültigkeit besitzt oder sich auch auf die Abhängigkeitsschwelle bezieht.

Obwohl ein nachfolgender Absatz die letzte These zu bestätigen scheint: "Bei der Berechnung der ... Schwellenwerte (Abhängigkeitsschwelle) bleiben die Erlöse aus der Ausfuhr der Bergbauerzeugnisse, die nicht unter das System fallen, unberücksichtigt" (Lomé IV: Art. 215 Abs. 1), ist diese Interpretation unwahrscheinlich. Zum einen würde daraus eine Schlechterstellung der AKP-Staaten gegenüber der vorherigen Regelungen in Lomé II und Lomé III resultieren, zum anderen widerspräche es der

1 Vgl. Lomé IV: Art. 215 Abs. 1. 
erklärten Absicht der Europäischen Gemeinschaft, die entwicklungspolitischen Aspekte gegenüber den rohstoffpolitischen Aspekten stärker zu gewichten.

Doch auch im Hinblick auf die zuletzt getroffene Aussage bietet der diskutierte Passus verschiedene Interpretationsvarianten. So bleibt es nach wie vor unklar, ob allein Ausfuhrrückgänge nach der Europäischen Gemeinschaft zu bewerten sind oder ob der Erlösrückgang bei denjenigen Produkten zu berücksichtigen ist, welche unter anderem auch nach der Europäischen Gemeinschaft exportiert werden - dort jedoch keine Einbuße erfahren haben. Die im Text vorgenommene Erweiterung auf "... der gesamten Ausfuhrerlöse des dem Jahr der Antragstellung vorangegangenen Jahres ..." (Lomé IV. Art. 215 Abs. 2b) bringt in diesem Falle auch keine Klarstellung. (Anm.: In Lomé IV gilt der Rückgang der Exporterlöse nur noch für die Fälle, wo die Abhängigkeitsschwelle aus der Ausfuhr eines Sysmin-Bergbauerzeugnisses erreicht wird (vgl. Lomé IV: Art. 215 Abs. 2b; s. Kap. 2.5.3)).

Da diese mangelnde Eindeutigkeit auch in der Lomé III-Version existierte, ${ }^{2}$ wird hier die Diskussion aufgegriffen, die bereits während der Laufzeit von Lome III geführt wurde:

Die europäischen Vertragspartner interpretierten den Passus in dem Sinne, daß als Auslösekriterium allein Erlösrückgänge bei Exporten nach der Gemeinschaft zu berücksichtigen seien. ${ }^{3}$ Die verantwortlichen Stellen in der EG-Kommission und dem Bundesministerium für Wirtschaft stützen ihre Version auf systematische, historische und teleologische Überlegungen, welche in die Verhandlungen zu den Lome-Abkommen mit eingeflossen seien. ${ }^{4}$

Da die Sysmin-Regelung in Anlehnung an das Stabex-Verfahren konzipiert wurde, ist der Kontext (systematische Interpretation), die Entstehungsgeschichte (historische Interpretation) und der Sinn und Zweck (teleologische Interpretation) eng mit diesem System verknüpft.

2 Vgl. Lomé III: Art 179 Abs. 1a.

3 Persönliche Gespräche, Kommission der EG, Direktion VIII, Brüsssel; Bundesministerium für Wirtschaft, Bonn; Institut für Internationales Recht, Universität Tübingen.

4 Persönliches Gespräch, Rechtsabteilung des Bundesministeriums für Wirtschaft, Bonn. 
Auch bei der Stabex-Regelung erwuchs ein Darlehensanspruch zunächst nur, wenn die Erlöse der Exporte nach der Gemeinschaft zurückgingen. ${ }^{5}$ Ausnahmeregelungen wurden erst in Lome III eingefuihrt und bedurften individueller Beschlüsse des Ministerrates (Lomé III: Art. 162, Art. 150). Erst in Lomé IV ist bei dem Stabex-Verfahren eine Automatik für Produkte eingefiihrt worden, die nicht nach der Europäischen Gemeinschaft exportiert werden (Lomé IV: Art. 189 Abs. 3).

In den Stabex-Regelungen wurde die Beschränkung auf den Exporterlösrückgang agrarischer Erzeugnisse nach der Europäischen Gemeinschaft vorgenommen,

- weil in Europa die statistische Erfassung ausgereifter und genauer ist als in vielen AKP-Staaten. Dadurch war und ist es schneller und umfassender möglich, eventuelle Rückgänge und deren Ausmaße festzustellen;

- weil die EG-Staaten zunächst wenig Bereitschaft zeigten, für Ausfälle zu zahlen, deren Ursachen nicht im Einflußbereich der Lomé-Vertragspartner begründet waren.

Hinsichtlich des Zusammenhanges (systematischer Rahmen) wurde der einleitende Artikel des Lomé-III-Sysmin-Verfahrens (Lome III: Art. 176, Lomé II: Art. 49) aufgeführt: "... um insbesondere diesen Staaten zu helfen, der Verringerung ihrer Kapazität zur Ausfuhr von Bergbauerzeugnissen nach der Gemeinschaft und der entsprechenden Verringerung ihrer Ausfuhrerlöse entgegenzuwirken ...". Auch hieraus - so wurde argumentiert - sei ersichtlich, daß sich das Interesse primär auf den Export von mineralischen Rohstoffen nach der Europäischen Gemeinschaft richte.

Die zweite Interpretationsmöglichkeit, d.h. den generellen Exportrückgang zu berücksichtigen, ließ sich in Lomé III durch die Satzkonstruktion (grammatikalischer Zusammenhang) begründen, die in den einzelnen Übersetzungen (englisch, französisch, deutsch) identisch war und somit nicht auf übersetzungstechnischen Notwendigkeiten beruhte. ${ }^{6}$ Durch die im Satzaufbau vorgenommene Trennung der Begriffe "Ausfuhrerlöse" und "nach der Gemeinschaft" - dies kommt in Lomé III durch den Ein-

5 Vgl. UNCTAD (Hrsg.): Compensory financing of export earnings shortfalls, a.a.O., S. 54.

6 Vgl. Lomé III: Art. 179 Abs. $1 \mathrm{a}$. 
schub eines Absatzes noch deutlicher zum Ausdruck - erscheint auch kein inhaltlicher Zusammenhang zwischen den Satzteilen zu bestehen (s. Anhang 4). Dies hätte zur Folge, daß die Berechnung der Ausfuhrerlösrückgänge nicht auf Exporte nach der Europäischen Gemeinschaft zu beschränken wäre.

Im Hinblick darauf, daß gemäß dieser Version auch Erlösrückgänge bei Ausfuhren nach anderen Ländern geltend gemacht werden könnten - wodurch der notwendige Prozentsatz der Auslöseschwelle schneller zu erreichen wäre -, kam diese Interpretation der entwicklungspolitischen Konzeption der Lome-Abkommen entgegen. Denn auf diese Weise konnte den betroffenen AKP-Staaten Unterstützung gewährt werden, ohne Unterschied, ob der Exporterlösrückgang durch die Europäische Gemeinschaft bedingt war oder nicht.

In Gesprächen wiesen Vertreter des Bundesministeriums für Wirtschaft darauf hin, daß bei den Sysmin-Verhandlungen eine möglichst große Parallelität zur Stabex-Regelung angestrebt worden sei. Daher seien bei dem Sysmin-Verfahren zunächst nur dort Abweichungen gegenüber der StabexRegelung vereinbart worden, wo spezielle Belange der Bergbauprodukte dies erforderlich machten. Da im Falle der Bewertung von Ausfuhrerlösen eine solche Modifikation nicht für nötig erachtet wurde, sahen die Verhandlungspartner bei der Konzeption des Sysmin-Verfahrens keinen Anlaß, von dem Stabex-Prozedere, welches sich bereits während Lomé I bewährt hatte, abzuweichen.

Jedoch weist die Rechtsabteilung des Bundesministeriums für Wirtschaft die Zweideutigkeit der Klausel im Lomé-III-Abkommen nicht grundsätzlich zurück. Insofern wäre es nur folgerichtig, daß im Hinblick auf die Erweiterung der Stabex-Auslöseschwelle auch die Auslöseschwelle des Sysmin-Verfahrens auf die gesamten Exporterlöse eines AKP-Staates für die jeweiligen Bergbauerzeugnisse ausgedehnt würde. Die grammatikalische Trennung zwischen dem anfangs zitierten Passus: "... die AKP. Staaten, deren Ausfuhren in die Gemeinschaft gehen ..." und der Auslöseschwelle (Lomé IV: Art. 215 Abs. 2; s. Anhang 4) scheint diese These zu bestätigen, zumal, wie erwähnt, im Zusammenhang mit der 15-prozentigen Abhängigkeitsschwelle von einem "Rückgang von mindestens 10 Prozent der gesamten Ausfuhrerlöse ..." gesprochen wird (Lome IV: Art. 215 Abs. 2b). Weshalb der Passus, "deren Ausfuhren in die Gemeinschaft gehen", dann aber überhaupt aufgenommen wurde, läßt sich bei dieser Interpretation nicht beantworten. In der Literatur - auch in 
Veröffentlichungen hinsichtlich der Lomé-IV-Verhandlungen - ist diese Problematik bisher noch nicht erörtert worden. Die Vermutung liegt nahe, daß das Interesse der Autoren allein der Gesamtkonzeption der Abkommen sowie den politischen Auswirkungen galt, Detailfragen aber - auch angesichts der in der Sysmin-Regelung vorgesehenen Von-Fall-zu-FallEntscheidungen - nicht näher untersucht wurden.

Auch seitens der AKP-Delegation sind bei den Lomé-IV-Verhandlungen keine Ändenungsvorschläge im Sinne der Gesamtexportvariante vorgetragen worden. Diese Tatsache erklärt sich im wesentlichen aus der, selbst für die AKP-Staaten geringen Relevanz dieser Version. Zum einen exportieren die von der Sysmin-Regelung erfaßten AKP-Staaten bereits zum überwiegenden Teil Bergbauerzeugnisse nach der Europäischen Gemeinschaft, zum anderen waren die bisherigen Sysmin-Anträge auch anders als durch einen Rückgang der Exporterlöse zu begründen.

Darüber hinaus mag auch die - besonders in den kleineren AKP-Staaten häufig anzutreffende - unzureichende Kenntnis über den Inhalt der Vertragstexte eine Rolle spielen, daß in dieser Hinsicht keine Modifikation vorgeschlagen wurde. Diese Behauptung läßt sich durch folgendes Beispiel bekräftigen:

Im Juli 1983 richtete Botsuana (Mitunterzeichner des Lomé-II-Abkommens) an die EG-Kommission die Anfrage, wie das Sysmin-Verfahren auf weitere mineralische Rohstoffe ausgedehnt und ob auch u.a. Kupfer aufgenommen werden könne. ${ }^{7}$ Beide Fragen hätten sich durch einen Blick in Artikel 50 des Lomé-II-Abkommens von selbst geklärt: Kupfer gehörte bereits zu den mineralischen Rohstoffen, auf welche das Sysmin-Verfah-' ren anwendbar war, und nach Artikel 50 Abs. 3 (Lomé III: Art. 177) bestand die Möglichkeit, auf Antrag weitere mineralische Rohstoffe aufzunehmen. Seit Lomé IV ist eine solche Erweiterungsmöglichkeit nicht mehr vorgesehen.

Die Regelung der Sysmin-Auslöseschwelle war in Lomé III gegenüber Lomé Il erweitert worden, indem - neben dem Unvermögen, die Produktionsanlagen oder die Ausfuhrkapazität zu erneuern - auch die Unterbrechung der Finanzierung großer Entwicklungsvorhaben zur Antragstellung berechtigte (Lomé III: Art. 179 Abs. 1). Durch einen Fehler jedoch wurde

7 Vgl. Kommission der EG (Hrsg.): Amtsblatt der EG (franz. Vers.), C 271, 26. Jg., 8. Oktober 1983, S. 18. 
der neue Passus kumulativ ("und" bzw. "and"; s. Anhang 4) statt fakultativ ("oder" bzw. "or") in den Artikel aufgenommen, so daß die Regelung in Lome III fälschlicherweise ${ }^{8}$ eine Nichterneuerung der Produktionsanlagen oder Ausfuhrkapazität und eine Unterbrechung großer Entwicklungsvorhaben vorgeschrieben hat. Diese Einschränkung der Anwendbarkeit ist in Lomé IV insoweit wieder aufgehoben worden, als die Unterbrechung von Entwicklungsvorhaben oder -programmen nur solche AKP-Staaten zur Antragstellung berechtigt, die mindestens 15 Prozent ihrer Exporterlöse durch eines der Sysmin-Bergbauerzeugnisse erwirtschaften (Lome IV: Art. 215 Abs. 2b). Die Regelung gilt für diese Gruppe von AKP-Staaten fakultativ zu den Auslösekriterien, die auch für diejenigen AKP-Staaten zutreffen, die mindestens 20 Prozent ihrer Erlöse durch die Ausfuhr verschiedener Bergbauerzeugnisse erzielen, nämlich die Feststellung der ernsthaften Gefährdung der Lebensfähigkeit von Bergbauunternehmungen, wobei ein Rückgang der Produktions- oder Ausfuhrkapazität um etwa zehn Prozent maßgebend ist (Lomé IV: Art. 215 Abs. 2a; s. Abb. 6).

In den Sysmin-Regelungen von Lomé II und Lomé III war das Auslösekriterium "Rückgang der Ausfuhrerlöse" nicht quantifiziert worden (Lomé III: Art. 179 Abs. 1). Darin lag ein wesentlicher Unterschied gegenüber der Stabex-Konzeption, wo der Rückgang der Exporterlőse als Auslösekriterium exakt definiert ist (Lomé III: Art. 162 Abs. 1). Det Schwerpunkt der Sysmin-Antragsberechtigung basiert auf dem Rückgang der Produktions- und Ausfuhrkapazitäten, das Erfassen der Ausfuhrerläse war lediglich eine Konzession an die AKP-Staaten, als die mineralischen Rohstoffe bei der Etablierung des Sysmin-Verfahrens aus der Stabex-Regelung ausgegliedert wurden. ${ }^{9}$

Seit Lomé IV ist dieses Kriterium nur noch für diejenigen AKP-Staaten relevant, die 15 Prozent ihrer Exporterlöse durch einen Sysmin-Rohstoff erwirtschaften. Es ist nun aber explizit festgehalten worden, daß es sich um einen mindestens zehnprozentigen Rückgang handeln muß, bezogen auf die gesamten Exporterlöse des betreffenden Bergbauerzeugnisses in dem Jahr, welches dem Jahr der Antragstellung vorangegangen ist (Lomé IV: Art. 215 Abs. 2b).

8 Persönliches Gespräch, Kommission der EG, Generaldirektion Entwicklung, Brüssel.

9 Persönliches Gespräch, Bundesministerium für Wirtschaft, Referat EA 6 , Bonn. 
Darüber hinaus ist in Lomé IV für alle antragsberechtigten AKP-Staaten (15 Prozent Erlöse Sysmin-Erzeugnis und 20 Prozent Erlöse sämtlicher Rohstoffe) neben dem Rückgang der Einkünfte - maßgebend ist nach wie vor ein etwa zehnprozentiger Rückgang der Produktions- oder Ausfuhrkapazität ${ }^{10}$ - nunmehr die Verschlechterung der Leistungsbilanz als weiteres Kriterium aufgenommen worden.

Dieses Kriterium gilt, im Gegensatz zu der Ausfuhrerlösregelung in Lomé II und Lomé III nicht mehr allein fakultativ (oder), sondern kann durch die Erweiterung und/oder auch kumulativ zur Anwendung kommen (Lomé IV: Art. 215 Abs. 2a).

lnwieweit diese neue Variante praktikabel ist, wird sich bei kommenden Anwendungsfällen zeigen. Durch die Erweiterung um das kumulative Element wird jedoch deutlich, daß eine offensichtliche Schwerpunktverlagerung auf das Kriterium des Kapazitätsrückganges vorgenommen wurde.

Trotz dieser Modifikationen wird an dieser Stelle die Forderung erhoben, bei einer zukünftigen Neuregelung der Sysmin-Artikel auf das Auslösekriterium "Leistungsbilanzverschlechterung" völlig zu verzichten. Eventuelle Unstimmigkeiten im Hinblick auf Interpretationsdifferenzen werden auf diese Weise vermieden, ohne daß eine Beschränkung der AKP-Interessen erfolgt, denn die verbleibenden Auslösefaktoren erfassen in hinreichendem Maße auch solche AKP-Staaten, die unter einer Leistungsbilanzverschlechterung leiden.

$\mathrm{Da}$ es auch durch weitere Umformulierungen nicht hätte vermieden werden können, daß es gelegentlich zu Manipulierungsversuchen kommen könnte, ${ }^{11}$ um Sysmin-Mittel zu erhalten, hatte man sich bei den Verhandlungen zu Lomé IV entschlossen, explizit auf die Unzulässigkeit eines derartigen Agierens hinzuweisen. Es wird daher sowohl in der generellen Auslöseklausel als auch in der speziellen Auslöseklausel für die AKPStaaten, die 15 Prozent ihrer Erlöse aus der Ausfuhr eines Sysmin-Erzeugnisses erwirtschaften, festgestellt, daß die Beeinträchtigung der Lebensfähigkeit nicht durch (künstliche) Maßnahmen des jeweiligen Staates

10 In der englischen Fassung wird von Kapazitäten (production or export capacities) ausgegangen.

1 Ob bereits Manipulationsversuche seitens der AKP-Antragssteller unternommen worden sind, um Sysmin-Mittel zu erhalten, die ihnen ansonsten nicht zugestanden hätten, konnte nicht verifiziert werden. 
beziehungsweise Unternehmens bedingt sein darf (Lomé IV: Art. 215 Abs. $2 a+2 b$ ).

Durch diese Maßnahme wurde die bereits auch in der Präambel der Sysmin-Regelung des Lomé-III-Abkommens enthaltende Formulierung: "... und vom Willen der betroffenen AKP-Staaten unabhängigen schweren Störungen ..." (Lomé III: Art. 176) nochmals deutlich hervorgehoben und die Absicht der Europäer, keine mutwillig zugrundegewirtschafteten Industrien zu unterstützen, klar herausgestellt.

\subsection{Die Leistungen unter der Sysmin-Regelung}

Die erste Tranche aus dem neugeschaffenen Sysmin-Fonds des Lome-IIAbkommens wurde im April 1982 in Höhe von 55 Mio. ECU an Sambia vergeben, zur Unterstützung der Rehabilitationsanstrengungen der Zambia Consolidated Copper Mines (s. Kap. 4.3.1).

Im gleichen Jahr wurde ein Darlehen aus dem Sysmin-Fonds in Höhe von 40 Mio. ECU an Zaire genehmigt, zur Wiederherstellung der Produktionsfazilitäten der Gécamines.

Für die notleidende Bauxit- und Aluminium-Industrie (Guymine) in Guayana ist im Jahre 1984 zunächst eine Vorauszahlung in Höhe von 3 Mio. ECU bewilligt worden. Erst nachdem ein für die EG-Kommission akzeptables Rehabilitationskonzept vorlag, erhielt Guayana für das betroffene Bergbauunternehmen ein Gesamtdarlehen in Höhe von 31,5 Mio. ECU.

Ruanda sind Ende des Jahres 1984 zunächst 2,84 Mio. ECU in einer ersten Rate für den Zinnbergbau zur Verfügung gestellt worden. Nach Vorlage eines Konzeptes zur Unterstützung des kleineren und mittleren Zinnbergbaues wurde der Gesamtbetrag in Höhe von 12 Mio. ECU genehmigt.

Zwei weiteren Anträgen aus Sambia und Zaire zur Fortsetzung der Rehabilitationsbemühungen für den Kupfer- und Kobaltbergbau wurde im Oktober 1985 mit 28 Mio. ECU für Sambia beziehungsweise Ende des Jahres 1986 mit 41 Mio. ECU für Zaire stattgegeben. Da der Antrag Zaires bereits im Jahre 1985 für zulässig erklärt worden war, wurde er noch aus dem Sysmin-Fonds des Lomé-II-Abkommens finanziert. 
Gleiches gilt für Anträge aus Liberia (Eisenerze) sowie Surinam und Jamaika (Bauxit), die vor dem Stichtag 31. Oktober 1985 bei der EG-Kommission in Brüssel eingegangen waren. Durch diese Regelung wurde erreicht, daß die Mittel der Sysmin-Fazilität des Lome-II-Abkommens voll ausgeschöpft worden sind und die im Text des Abkommens vorgesehene Maßnahme: Der Ministerrat entscheidet über die Verwendung der Restbestände (Lomê II: Art. 51 Abs. 2), nicht zur Anwendung kommen mußte.

Liberia wurden im Oktober 1987 eine Finanzierungsbewilligung in Höhe von 49,3 Mio. ECU erteilt, womit der wirtschaftliche Abbaubetrieb der Bong Mining Company wiederhergestellt werden sollte. Die Gesamtrehabilitierungskosten belaufen sich auf 104,3 Mio. ECU. Im Januar 1989 wurden Guinea 35 Mio. ECU für die Modernisierung der im Jahre 1960 erōffneten Aluminiumhütte Friguia genehmigt. ${ }^{12}$

Die während der Laufzeit von Lomé III gestellten Anträge auf Darlehen aus der Sysmin-Fazilität basierten auf den Anspruchsgrundlagen (Abhängigkeit) sowohl des Artikel 180 Abs. a (15 Prozent der Exporterlöse durch einen Sysmin-Rohstoff) als auch des Artikels 180 Abs. b (20 Prozent der Erlöse aus der Ausfuhr sämtlicher Bergbauerzeugnisse). ${ }^{13}$

Im Februar 1987 wurde das erste Darlehen nach Artikel 180 Abs. b in Höhe von 12,5 Mio. ECU an Niger genehmigt. Eine weitere Novität bei dieser Mittelvergabe betraf den Verwendungszweck. Erstmalig war vereinbart worden, daß die Darlehen für Explorationsvorhaben nach Goldund Kohlelagerstätten eingesetzt werden und nicht bei der Rehabilitation bestehender Produktionsfazilitäten Verwendung finden. ${ }^{14}$

12 Persönliches Gespräch, Kommission der EG, Brüssel; Vgl. O.V.: Financing of mining projects, in: The Courier, Nr. 109, Mai - Juni 1988, S. 13; O.V.: Bauxit-Darlehen für Guayana, in: BfA/NfA, 15. November 1988; O.V.: Sysmin, in: The Courier, Nr. 113, a.a.O., S. XI f.

13 Vgl. Rechnungshof der EG (Hrsg.): Jahresbericht zum Haushaltsjahr 1985, in: Amtsblatt der EG, C 321, 29. Jg., 15. Dezember 1986, S. 145; Kommission der EG (Hrsg.): Amtsblatt der EG, C 177, 30. Jg., 6. Juli 1987, S. 57 f.

14 O.V.: Sysmin: Niger; Research into coal and goldmining, in: The Courier, Nr. 111, September - Oktober 1988, S. XI. 
Im Falle Papua-Neuguineas wurden 18 Mio. ECU für die Instandsetzung von Straßen und Brücken eingesetzt, deren Unterhaltung aufgrund fehlender Mittel nicht in erforderlichem Maße aufrechterhalten werden konnte. ${ }^{15}$

Für die Phosphatindustrie Togos ist im Juli 1988 eine Sysmin-Tranche in Höhe von 15,7 Mio. ECU genehmigt worden. Die Mittel werden eingesetzt, um Lösungsmöglichkeiten zur Dekontamination von cadmiumhaltigen Phosphaten zu untersuchen. ${ }^{16}$

Im Sommer 1989 bewilligte die EG-Kommission Botswana ein Investitionsprogramm in Höhe von insgesamt 40,8 Mio. ECU zur Verwendung in der Nickel- und Kupferindustrie; 21,7 Mio. ECU davon werden durch die Sysmin-Fazilităt finanziert.

Neben der Aufrechterhaltung der Produktionsfazilitäten der Bamangwato Concession Ltd. (BCL), dem größten privaten Arbeitgeber des Landes, sollen vor allem Explorationsvorhaben ausgeführt werden. ${ }^{17}$

Im Jahre 1991 wurde einem Antrag Senegals auf Unterstützung seiner Phosphatindustrie stattgegeben. Die 15 Mio. ECU aus der Sysmin-Fazilität werden ähnlich wie in dem Projekt Togos für ein mehrstufiges Programm zur Untersuchung und Reduzierung des umweltrelevanten $\mathrm{Cad}$ miumgehaltes im Phosphat eingesetzt. Darüber hinaus sollen Rationalisierungsmaßnahmen helfen, Kosten zu senken, um so die Gesamtwirtschaftlichkeit trotz der zusätzlichen Kosten für die spätere Cadmiumreinigung zu gewährleisten. ${ }^{18}$

Im Jahre 1992 wurden zum dritten Male Sysmin-Mittel an die ZCCM in Sambia bewilligt. Bei den Mitteln in Höhe von 40 Mio ECU, die für ge-

15 Vgl. Loftus, J.: Cooperation between Papua New Guinea and the European Comunity, in: The Courier, Nr. 133, Mai - Juni 1992, S. $37 \mathrm{f}$.

16 Vgl. O.V.: Sysmin-Phosphate mining industry, in: The Courier, Nr. 119, Januar - Februar 1990, S. II gelb.

17 Vgl. O.V.: Sysmin, Botswana; in: The Courier, Nr. 117, September Oktober 1989, S. IV f. gelb.

18 Vgl. O.V.: Sysmin, Senegal; in: The Courier, Nr. 125, Januar - Februar 1991, S. IV gelb; O.V.: Sysmin, ECU 15.000 .000 to help Senegal solve the problem of cadmium, in: The Courier, Nr. 126, März - April 1991, S. IX gelb. 
nerelle Importe einzusetzen sind handelt es sich erstmals für Sambia um Zuschüsse gemäß der Sysmin-Regelung in Lomé IV. ${ }^{19}$

Im März 1993 wurde zur Unterstützung der Bergbauindustrie Namibias ein Zuschuß aus der Sysmin-Fazilität in Höhe von 40 Mio ECU bewilligt. ${ }^{20}$

\subsection{Die Analyse der Durchführbarkeit der Sysmin-Regelung anhand von zwei Fallbeispielen}

In den beiden folgenden Kapiteln wird näher auf zwei AKP-Staaten eingegangen, die als typische Vertreter der AKP-Bergbaustaaten anzusehen sind.

Bereits der Vergleich zwischen der wirtschaftlichen Situation in Sambia und der in Simbabwe läßt deutlich werden, daß eine Übertragung von Lösungsansätzen, die in einem Lande Erfolge zeitigten, nicht ohne weiteres sinnvoll ist, selbst wenn es sich um die gleichen Sektoren, wie hier den Bergbausektor handelt.

Für diese Gegenüberstellung wurden die Bergbauländer Sambia und Simbabwe ausgewählt, weil die Gemeinsamkeiten auf den ersten Blick zu überwiegen scheinen. Beide Staaten liegen im südlichen Zentralafrika, sind sogar unmittelbare Nachbarn. Viele Jahre, als beide Regionen in der britischen Kolonie Rhodesien zusammengefaßt waren, wurden sie durch ein Kolonialsystem verwaltet.

Obwohl beide Staaten ein großes Geopotential besitzen, haben sich die Gemeinsamkeiten mit obiger Aufzählung bereits erschöpft. Bereits in der Abhängigkeitsschwelle der Sysmin-Regelung unterscheiden sich die beiden Nachbarstaaten: Während Sambia mehr als 15 Prozent seiner Exporterlöse durch die Ausfuhr von Kupfer beziehungsweise Kobalt erwirtschaftet, exportiert Simbabwe eine Vielzahl von Bergbauprodukten und hat erst aufgrund der seit Lomé III bestehenden 20-Prozent-

19 Vgl. O.V.: The Convention at work, in: The Courier, Nr. 137, Januar - Februar 1993, S. II gelb.

20 Vgl. O.V.: The Convention at work, in: The Courier, Nr. 139, MaiJuni 1993, S. I gelb. 
Abhängigkeitsschwelle die Möglichkeit, Anträge auf Sysmin-Mittel zu stellen. Während seitens Simbabwe beziehungsweise der dort ansässigen Bergbauindustrie bisher noch keine Absichten auf Stellung eines SysminAntrages bekannt geworden sind, hat Sambia bereits unter Lomé II erste Sysmin-Darlehen erhalten; ein dritter Antrag wurde im Dezember 1992 bewilligt und wird gemäß der Sysmin-Regelung in Lomé IV als Zuschuß ausgezahlt.

So wird anhand des Vergleiches dieser benachbarten Bergbaustaaten deutlich, daß Konzepte zur Lösung von eventuellen Schwierigkeiten in den jeweiligen AKP-Bergbauindustrien nur effizient sein können, wenn Programme und Maßnahmen hinreichend individuell einsetzbar sind. Dies gilt in besonderem Maße auch für die Sysmin-Regelung, denn der flexible Einsatz von Mitteln ist, wie die Begehungen von Bergbaubetrieben deutlich machten, besonders im Bergbau erforderlich, da nicht nur die einzelnen Länder, sondern auch die einzelnen Rohstoffe sowie die jeweiligen Bergwerke ihre charakteristischen Besonderheiten aufweisen.

\subsubsection{Fallbeispiel Sambia}

Die Republik Sambia erstreckt sich im südlichen Zentralafrika über eine Fläche von über $750.000 \mathrm{~km}^{2}$ und grenzt an 8 andere afrikanische Staaten (s. Karte 1). Im Süden: Mosambik, Simbabwe, Botswana und Namibia, im Westen: Angola, im Norden: Zaire und im Osten: Tansania und Malawi.

Somit verfügt Sambia über keine eigene Küste und ist daher bei sämtlichen Übersee-im- und -exporten (vom Luftwege abgesehen) auf die Transportkapazitäten der Nachbarstaaten sowie deren Transfererlaubnis angewiesen.

Obgleich sich mehrere große Seen im Land befinden, darunter der Tanganjika- und der Kariba-See, gibt es keine schiffbaren Flüsse. 


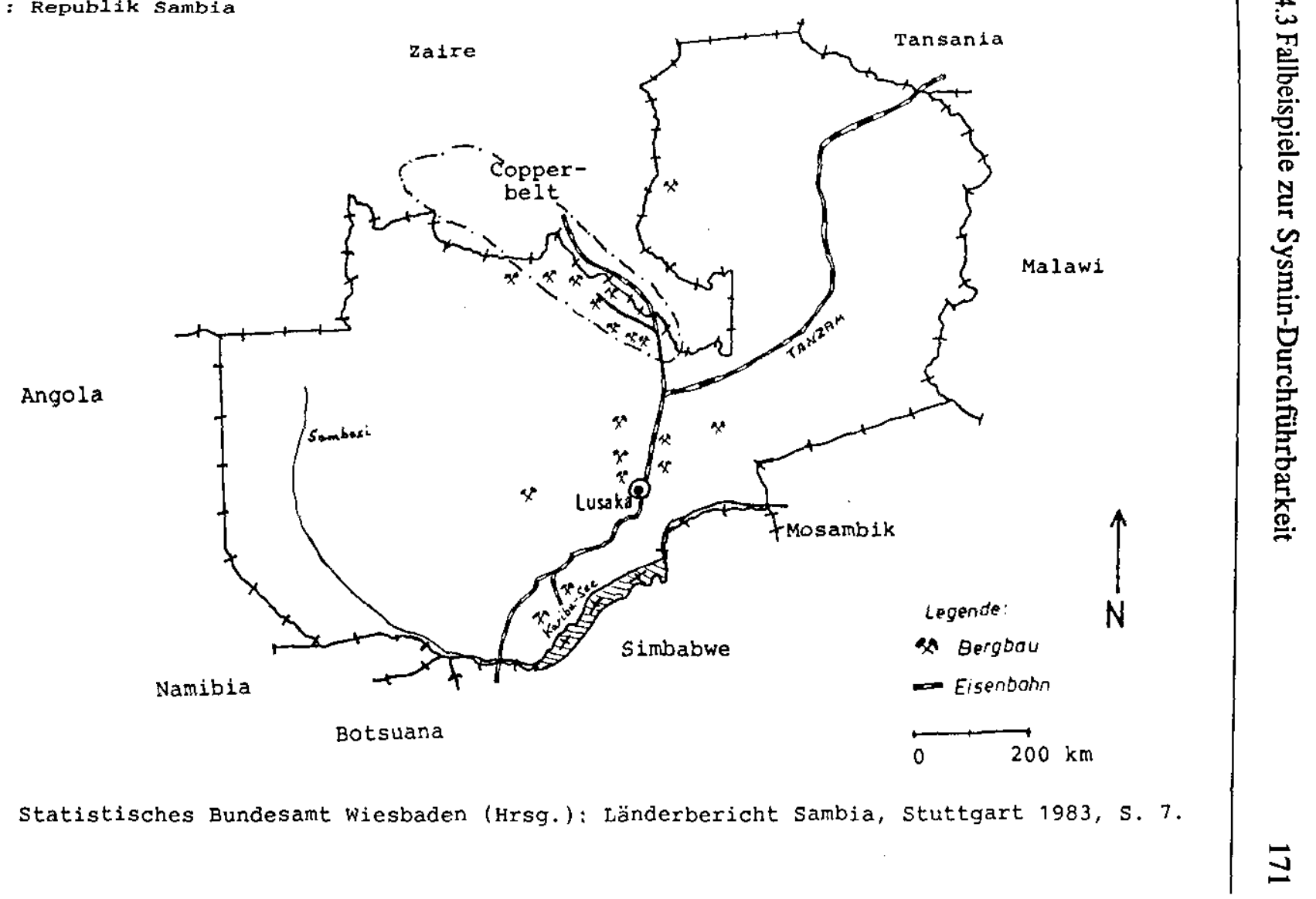


Das Klima Sambias kann aufgrund der durchschnittlichen Höhe von 1000 bis $1500 \mathrm{~m}$ über dem Meeresspiegel als subtropisch und gemäßigt bezeichnet werden. Fruchtbarer Boden und eine ausreichende Wasserversorgung - sowohl durch Niederschläge als auch durch Wasserläufe erlauben eine ganzjährige Feldbestellung. Trotzdem werden gegenwärtig nur etwa 8 Prozent der Fläche Sambias landwirtschaftlich intensiv genutzt, obwohl sich dafür rund 50 bis 60 Prozent des Landes eignen würden. ${ }^{21}$

Die Bevölkerung setzt sich aus etwa 70 verschiedenen Stammesgruppen zusammen und wird auf 7 Mio. Einwohner geschätzt. Dabei ist anzumerken, daß über 65 Prozent (1977) der Sambier unter 25 Jahre alt sind. Die durchschnittliche Wachstumsrate der Bevölkerung wird mit etwa 3,6 Prozent pro Jahr angegeben. Neben den schwarzen Einwohnern leben in Sambia noch etwa 40.000 Weiße und 30.000 Menschen asiatischer Abstammung.

Auf $1 \mathrm{~km}^{2}$ siedeln durchschnittlich 8 Personen, wobei die Bevölkerungsdichte jedoch stark variiert. Nahezu die Hālfte der Bewohner Sambias leben in Städten, besonders entlang der Eisenbahnlinie Livingstone - Lusaka - Ndola. Die Landflucht schafft in den Städten erhebliche Probleme; so sind z.B. in Lusaka etwa 50 Prozent der männlichen Bewohner ohne Beschäftigung. ${ }^{22}$

Die ehemalige Kolonie Nord-Rhodesien ist am 24. Oktober 1964 unabhängig geworden. Der erste Präsident Kaunda bekleidete dieses Amt bis zum Novernber 1991. Im Jahre 1972 wurde eine Einheitspartei errichtet, die United National Independence Party, UNIP. Die politischen Aktionen der UNIP sind angabegemäß geprägt von "christlichem Humanismus und afrikanischem Sozialismus". Der durch eine demokratische Wahl an die Macht gekommene Nachfolger Kaundas, Chiluba will das Einparteiensystem durch ein Mehrparteiensystem ablösen. ${ }^{23}$

2] O.V.: Mühsame Sanierung der Wirtschaft Sambias, in: Blick durch die Wirtschaft, 30. April 1986.

22 Persönliche Gespräche, Bank of Zambia, Lusaka, Deutsche Botschaft, Lusaka 1984 und 1988; Vgl. Statistisches Bundesamt Wiesbaden (Hrsg.): Länderbericht Sambia, Stuttgart 1985, S. 15 f.; Bundesstelle für Außenhandelsinformation (Hrsg.): Sambia, September 1988, S. 1.

23 Vgl. Krabbe, G.: Chiluba steht in Sambia vor einer schweren Aufgabe, in: Frankfurter Allgemeine Zeitung, 4. November 1991. 
Sambia ist u.a. Mitglied in den Vereinten Nationen, des Internationalen Währungsfonds, der Organisation für Afrikanische Einheit (OAU) und über die AKP-EWG-Abkommen von Lomé mit der Europäischen Gemeinschaft assoziiert. Allein die Bundesrepublik Deutschland hat dem Land in dem Zeitraum von 1950 bis zum 31.12.1987 Mittel in Höhe von 650 Mio. DM bewilligt, davon 482 Mio. DM für die Finanzielle Zusammenarbeit und 168 Mio. DM für die Technische Zusammenarbeit im engeren Sin$\mathrm{ne}^{24}$

Die Europäer haben Sambia ebenfalls erhebliche Mittel zur Verfügung gestellt. Seit Lomé I wurden in dem Zeitraum von 1975 bis Ende 1987 insgesamt 310,8 Mio. ECU für nationale Programme und Projekte bewilligt. Davon wurden 242,5 Mio. ECU aus EDF-Mitteln, 57,9 Mio. ECU von der EIB (einschließlich 15,9 Mio. EEF-Risikokapital) sowie 10,4 Mio. ECU aus dem Etat der Kommisson (u.a. Sonderprogramme) finanziert. Die Unterstützung regionaler Programme durch die Europäische Gemeinschaft, zum Beispiel die Ertüchtigung und Erneuerung der Tanzam-Eisenbahn, die ebenfalls einen direkten Einfluß auf die sambische Wirtschaft haben, sind hier nicht erfaßt. ${ }^{25}$

Derzeit erlebt Sambia die schwerste Wirtschaftskrise seit seiner Unabhängigkeit im Jahre 1964. Die Kupferproduktion reduzierte sich seit 1980 um mehr als 30 Prozent.

Nach dem im Mai 1987 erfolgten Bruch mit dem Internationalen Währungsfonds (IMF) sieht sich das Land zusätzlichen finanziellen Problemen gegenübergestellt, da die meisten der internationalen Kreditgeber (einschlieBlich Weltbank) die Bereitstellung weiterer Mittel von einer Einigung mit dem IMF abhängig machen. ${ }^{26}$

Die Konfrontation mit dem IMF erreichte ihren Höhepunkt, als Sambia eine Beschränkung des Schuldendienstes auf 10 Prozent der Deviseneinnahmen festlegte. Bereits zuvor hatte die sambische Regierung die Sub-

24 Persönliches Gespräch, Deutsche Botschaft, Lusaka 1989.

25 Delegation der Kommission der Europäischen Gemeinschaften (Hrsg.): Co-operation Zambia European Communities, Annual Report 1987, a.a.O., S. 8 f.

26 Nicht alle EG-Mitgliedstaaten verfolgen die gleiche strikte Linie; z.B. hat Italien im Februar 1989 der sambischen Regierung weitere finanzielle Unterstützung zugesagt. 
vention von Grundnahrungsmitteln wieder aufgenommen, welches dem gemäß den IMF-Richtlinien durchgeführten - Sanierungsprogramm entgegenstand. Diese Maßnahme war erforderlich geworden, nachdem als Folge des IMF-Programms eine Preisverdoppelung für das Grundnahrungsmittel Mais eingetreten war, wodurch erhebliche Unruhen unter der Bevölkerung ausgelöst wurden. Das Pro-Kopf-Einkommen ist in den Jahren 1980 - 1987 nach Angaben der sambischen Finanzbehörden um fast ein Fünftel gesunken, das BIP hat seit dem Jahre 1975 um über 30 Prozent abgenommen.

Die unbefriedigende Situation in der Landwirtschaft hat sich trotz einer Rekordernte im Jahre 1988 aufgrund günstiger Wetterbedingungen nur unwesentlich verbessert; die angekündigten Fördermaßnahmen wurden nur in geringem Umfange realisiert.

Seit Jahren leidet die verarbeitende Industrie unter einem Produktionsrückgang, bedingt durch die angespannte Devisensituation - trotz stark eingeschränktem Schuldendienst leidet Sambia unter großem Devisenmangel - und die drastischen Importbeschränkungen. Die Leistungsbilanz Sambias entwickelt sich seit Jahren ungünstig (s. Tab. 17), die hohe Auslandsverschuldung, die zum Teil noch aus den Zeiten einer blühenden Kupferindustrie stammt, belief sich Mitte 1988 auf etwa $6 \mathrm{Mrd}$. US-\$. Der theoretische Schuldendienst (allein Zinsen fast 295 Mio. US-\$ in 1988) übersteigt damit die Exporterlöse. Die Perspektiven für die kommenden Jahre sind düster. ${ }^{27}$

Die Wirtschaft Sambias ist von jeher in starkem Maße vom Bergbau abhängig (s. Tab. 18 und Anhang 2). Die Entwicklung der Bergbautätigkeit begann, als Sultane aus Sansibar und arabische Händler mit ihren Karáwanen durch das südliche Afrika zogen, um sich neben Sklaven mit Edelsteinen und -metallen zu versorgen.

27 Persönliche Gespräche, Deutsche Botschaft und EG-Delegation in Lusaka, Sambia 1989; vgl. O.V.: Mühsame Sanierung der Wirtschaft Sambias, a.a.O.; O.V.: An IMFallible Leader, in: The Economist, 10. Januar 1987; O.V.: Zambia: New economic policies aim to break vicious circle, in: Africa Economic Digest, 10. Juli 1987; O.V. Sambias Alleingang ohne viel Zukunft, in: Neue Zürcher Zeitung, 28. Januar 1988; O.V.: Desolate Verhältnisse in Sambia, in: Neue Zürcher Zeitung, 13. Februar 1988; Bundesstelle für Außenhandelsinformation (Hrsg.): Wirtschaftsentwicklung, Sambia, Januar 1989, S. $1 \mathrm{f}$. 
Tabelle 17: Sambias Leigtungsbilanz für aie Jahke 1987 wnd 1908 (in Mio, Kwacha)

\begin{tabular}{|c|c|c|c|c|c|}
\hline & \multicolumn{2}{|c|}{$\begin{array}{l}\text { Ausgaben } \\
\text { E=mm= }\end{array}$} & & \multicolumn{2}{|c|}{$\begin{array}{l}\text { Einnahmen } \\
===\equiv= \pm x=\end{array}$} \\
\hline & 1987 & 1988 & & 1987 & 1988 \\
\hline Importe (f.o.b.) & 5568,2 & 5723,4 & Exporte (f.o.b.) & 8058,6 & 9185,7 \\
\hline $\begin{array}{l}\text { davon: Rohöl } \\
\text { Dünger }\end{array}$ & & $\begin{array}{r}641,6 \\
48 ; 7\end{array}$ & $\begin{array}{l}\text { davon: } \\
\text { Kupfer } \\
\text { Kobalt } \\
\text { Blei } \\
\text { Elektrizität } \\
\text { Tabak }\end{array}$ & $\begin{array}{r}854,9 \\
466,2 \\
131,1 \\
19,6 \\
80,3 \\
16,6\end{array}$ & $\begin{array}{r}7884,8 \\
512,8 \\
157,5 \\
19,0 \\
95,4 \\
6,7\end{array}$ \\
\hline Handelsüberschus & & & & 2490,4 & 3462,3 \\
\hline $\begin{array}{l}\text { Fracht, Versicherung, } \\
\text { Transport } \\
\text { Reisekosten } \\
\text { Investitionen }\end{array}$ & $\begin{array}{ll}1 & 276,3 \\
437,0 \\
2 & 258,6\end{array} \mid$ & $\begin{array}{r}1491,5 \\
378,8 \\
3 \quad 36 \%, 1\end{array}$ & Tourismus & $\begin{array}{r}3+8,8 \\
60,0 \\
10,0\end{array}$ & $\begin{array}{r}352,6 \\
42,3 \\
10,0\end{array}$ \\
\hline Sonst. Dienstleistungen & & & & & \\
\hline $\begin{array}{l}\text { staatlich } \\
\text { privat }\end{array}$ & $\begin{array}{l}148,2 \\
261,2\end{array}$ & $\begin{array}{l}236,3 \\
239,7\end{array}$ & & $\begin{array}{r}70,0 \\
5,0\end{array}$ & $\begin{array}{r}50,0 \\
5,5\end{array}$ \\
\hline übertragungen & & & & & \\
\hline $\begin{array}{l}\text { staatlich } \\
\text { privat }\end{array}$ & $\begin{array}{r}12,5 \\
191,1\end{array}$ & $\begin{array}{r}29,9 \\
149,7\end{array}$ & & $\begin{array}{r}289,9 \\
5,0\end{array}$ & $\begin{array}{r}290,0 \\
5,0\end{array}$ \\
\hline Leistungsbilanzsaldo - & 1335,8 & $-1669,3$ & & & \\
\hline Kapitalbilanzsaldo & 7088,5 & n.v. & & & \\
\hline Devisenbilanzsaldo - & 286,7 & n.v. & & & \\
\hline zahlungsbilanzsaldo - & 8711,0 & n.v. & & & \\
\hline
\end{tabular}

Quelle: Bank of Zambia (Hrsg.): Central statistical office, 0.0., Februar 1989; Material teilweise unveröfentlicht. 
Im Jahre 1851 entdeckte Livingstone den Sambesi und erforschte dessen Verlauf in einer zwölfjährigen Expedition. Im letzten Jahrzehnt des 19. Jahrhunderts begann dann die große Kolonisationswelle der Europäer. Vertreter der British South Africa Company (BSAC) legten Handelsplätze an und beanspruchten die Gebiete Süd-Rhodesien (Simbabwe) und NordRhodesien (Sambia) für sich. Diese Region wurde dann im Jahre 1891 von Portugal, der zweiten Kolonialmacht in diesem Raume, als von der BSAC verwaltete Territorien anerkannt.

Nachdem jedoch die immensen Rohstoffvorkommen im Norden Rhodesiens immer offensichtlicher geworden waren, übernahm die britische Krone im Jahre 1924 die Verwaltungskontrolle. ${ }^{28}$

Die ersten Abbaukonzessionen im Kupfergürtel wurden im Jahre 1902 vergeben, der moderne Großbergbau begann allerdings erst, als im Jahre 1923 die Prospektions-, Explorations- und Abbaurechte einigen finanzkräftigen Bergbaugesellschaften zugebilligt wurden.

Aufgrund der überdurchschnittlich großen Vorkommen konzentrierte sich die Bergbautätigkeit auf die Gewinnung von Kupfer, Kobalt, Blei und Zink. Auch heute noch stellen diese vier mineralischen Rohstoffe den überwiegenden Teil der Bergbauerzeugnisse Sambias dar (s. Tab. 17 und 19). Diese Produkte werden auch in den kommenden Jahrzehnten die Haupteinnahmequelle Sambias sein, obwohl mittlerweile einige Lagerstätten erschöpft sind und sich der Abbau der Erze immer schwieriger und aufwendiger gestaltet.

Im Zuge der Unabhängigkeit Sambias wurden in den siebziger Jahren die beiden größten Bergbaugesellschaften Anglo American Corporation Ltd. und Roan Selection Trust Ltd. "reorganisiert" und in Nchanga Consolidated Copper Mines Ltd. (NCCM) und Roan Consolidated Mines Ltd. (RCM) umbenannt. Von diesen Gesellschaften übernahm der sambische Staat je 51 Prozent der Anteile, der Rest blieb in ausländischer Hand. ${ }^{29}$

28 Vgl. Bradley, K.: Copper Venture, London 1952, S. 77; Freemann, P.V.: Mining in Zambia today, Lusaka 1983, S. 1.

29 Vgl. Zambia Mining Yearbook 1980, Kitwe, o.J., S. 1 
Tabelle 18: Die Zusammensetzung des Bruttoinlandsproduktes von Sambia (in Prozent)

\begin{tabular}{|l|c|c|}
\hline \multirow{2}{*}{} & \multicolumn{2}{|c|}{ Sambia } \\
\cline { 2 - 3 } & 1980 & 1987 \\
\hline Primärer Sektor & 30,3 & 24,8 \\
\hline $\begin{array}{l}\text { Land- und Forstwirtschaft, } \\
\text { Fischerei } \\
\text { Bergbau }\end{array}$ & 14,2 & 11,2 \\
\hline Sekundärer Sektor & 16,1 & 13,6 \\
\hline $\begin{array}{l}\text { Weiterverarbeitende Industrie } \\
\text { Handel und Gastgewerbe }\end{array}$ & 28,5 & 48,0 \\
Tertiärer Sektor und Zolleinnahmen & 17,2 & 27,5 \\
\hline nicht erfaßte Bereiche & 34,7 & 24,2 \\
\hline
\end{tabular}

Quelle: Eigene Berechnung nach: Statistisches Bundesamt Wiesbaden (Hrsg.): Länderbericht Sambia, Stuttgart 1985, S. 84; Bank of Zambia (Hrsg.): Central statistical office, o.O., Februar 1989.

Im Jahre 1982 schließlich fusionierten die RCM und die NCCM zur Zambia Consolidated Copper Mines Ltd. (ZCCM), ${ }^{30}$ vorgeblich um Kosten einzusparen. ${ }^{31}$ Eine im Rahmen der Fusion vorgenommene Kapitalerhöhung sicherte der sambischen Regierung einen Anteil von 60,3 Prozent an

30 Vgl. Zambia Consolidated Copper Mines (Hrsg.): Annual Report 1982, a.a.O., S. 19.

31 Vgl. Zambia Consolidated Copper Mines (Hrsg.): Annual Report 1983, a.a.O., S. 4. 
der ZCCM, welcher von der staatlichen Zambia Industrial and Mining Corporation Ltd. (ZIMCO) verwaltet wird. ${ }^{32}$

Tabelle 19: Die Bergbauproduktion der ZCCM (in $1000 \mathrm{t}$ )

\begin{tabular}{|l|r|r|r|r|}
\hline & $1977 / 78$ & $1980 / 81$ & $1983 / 84$ & $1987 / 88$ \\
\hline & & & & \\
Kupfer* & 648,0 & 588,0 & 550,0 & 473,0 \\
Kobalt & 1,6 & 2,9 & 2,8 & 4,7 \\
Blei & 12,5 & 10,0 & 11,6 & 7,8 \\
Zink & 39,0 & 34,0 & 34,0 & 21,0 \\
\hline
\end{tabular}

Anm.: *) im wesentlichen Kupferkathoden; alle Angaben gerundet; jeweils Metallinhalt ZCCM-Geschäftsjahr 1. April bis 31. März.

Quelle: Eigene Zusammenstellung nach: Zambia Consolidated Copper Mines (Hrsg.): Annual Report 1982, Lusaka, S. 7 f., S. 34; Derselbe: Annual Report 1988, Lusaka, S. 9 f., S. 31.

Durch den Zusammenschluß der RCM und NCCM konnten zwar gewisse Kosten reduziert werden - z.B. durch Rationalisierungsmaßnahmen bei den Lagerbeständen -, ob dadurch aber die entstandenen Nachteile aufgewogen werden können, erscheint fraglich. So ist das neue Unternehmen wesentlich unflexibler geworden, die Verwaltung komplizierter, die Entscheidungswege länger. Ein Teil der ZCCM-Verwaltung wurde mittlerweile wieder aus dem etwa $350-400 \mathrm{~km}$ von den Produktionsstätten entfernten Lusaka in den Kupfergürtel zurückverlegt. ${ }^{33}$ Dennoch sehen Beobachter aus Europa in der ZCCM das zur Zeit am besten organisierte, sambische Unternehmen. ${ }^{34}$

32 Vgl. Smith, M.: ZCCM on course for recovery, in: Mining Magazine, Dezember 1987, S. 524.

33 Persönliche Gespräche, ZCCM-Abbaubetriebe; Vgl. Zambia Consolidated Copper Mines (Hrsg.): Annual Report 1986, a.a.O., S. 5.

34 Persönliches Gespräch, EG-Delegation, Lusaka, Sambia 1989. 
Besondere Nachteile bezüglich der internationalen Wettbewerbsfähigkeit ergeben sich für den sambischen Kupferbergbau sowohl aus den hohen Produktionskosten als auch aus den langen und kostenintensiven Transportwegen zu den internationalen Absatzmärkten (s. Tab. 20), die zudem noch durch politisch beziehungsweise wirtschaftlich instabile Staaten führen.

Tabelle 20: Abnehmerstaaten der ZCCM-Kupferproduktion

\begin{tabular}{|l|r|r|r|}
\hline Jahr & $1979 / 80$ & $1982 / 83$ & $1987 / 88$ \\
\hline Europäische Gemeinschaft & $50 \%$ & $56 \%$ & $40 \%$ \\
Japan & $21 \%$ & $20 \%$ & $27 \%$ \\
USA & $10 \%$ & $3 \%$ & $6 \%$ \\
sonst. Europa + Comecon & $7 \%$ & $12 \%$ & $6 \%$ \\
andere Staaten & $12 \%$ & $9 \%$ & $21 \%$ \\
\hline
\end{tabular}

Quelle: Eigene Berechnungen nach: Zambia Mining Yearbook 1980, Kitwe, S. 22; Zambia Consolidated Copper Mines (Hrsg.): Annual Report 1983, Lusaka, S. 23; Derselbe: Annual Report 1988, Lusaka, S. 22.

Im internationalen Vergleich gesehen entstehen dem Kupferbergbau im afrikanischen Kupfergürtel die höchsten Produktionskosten, die Kupferproduktion in Chile dagegen beispielsweise gehört weltweit zu den kostengünstigsten. ${ }^{35}$ Im Jahre 1985 beliefen sich die durchschnittlichen Produktionskosten (einschließlich der Kapitalkosten, abzüglich der Erträge für Kuppelprodukte) in Sambia angabegemäß auf etwa 1.640 US-\$/t Kupfer; sie konnten auf etwa 1.300 US-\$/t Kupfer im Jahre 1987 reduziert werden ${ }^{36}$ Die durchschnittlichen Produktionskosten der chilenischen Kupfergesellschaften im Jahre 1985 betrugen etwa 930 US-\$/t Kupfer. Die Kosten lagen damit auch deutlich unter denen der US-amerikanischen Kupferproduzenten (etwa 1.440 US-\$/t), obwohl auch in den USA u.a.

Vgl. O.V.: Copper - A Third World View, in: Mining journal, Vol. 304, Nr. 7813, 17. Mai 1985, S. 341, Commodities Research Unit (Hrsg.): Copper to 1995, Vol. III, London 1984, S. 25 f.

36 Persönliches Gespräch, ZCCM-Headoffice, Lusaka. 
durch Stillegung, Rationalisierung und Lohnabbau eine Kostenreduktion gegenüber dem Beginn der achtziger Jahre (etwa 1.980 US $\$$ /t) erzielt werden konnte. ${ }^{37}$ Der chilenische Kupferabbau arbeitet so kostengünstig, weil er im wesentlichen auf Großlagerstätten, die als Tagebaue betrieben werden können, beschränkt ist. Allein die Kupferreserven von zwei chilenischen Großlagerstätten übersteigen diejenigen der USA und der ehemaligen UdSSR.

Desweiteren kann der Kupferbergbau Chiles auf gute Anbindungen an die pazifischen Tiefwasserhäfen zurückgreifen. ${ }^{38}$ Darüber hinaus bestehen dort aufgrund der Ladekapazitäten und der großen Tonnagen, die zu verschiffen sind, geringere Frachtraten als beispielsweise in Papua-Neuguinea. ${ }^{39}$

Die Binnenlage Sambias verursacht nicht nur höhere Transportkosten (1980: etwa 170 US- $\$ / t$ Kupfer) ${ }^{40}$ im Vergleich zu Exporten aus Küstenoder Inselstaaten, sondern zwingt auch dazu, Transport- und Verladeeinrichtungen in politisch oder wirtschaftlich instabilen Regionen in Anspruch zu nehmen. Trotz umfangreicher Rehabilitationsprogramme auf den Eisenbahnstrecken nach Mosambik und Tansania (TANZAM) treter dort immer wieder Lieferverzögerungen ein..$^{41}$

Wie bereits dargelegt, wurden Sambia und Zaire die ersten Tranchen aus dem Sysmin-Fonds zugesprochen, 4 beziehungsweise 5 Jahre später sind erneut Sysmin-Darlehen an diese AKP-Staaten vergeben worden.

Gespräche in Sambia - mit Führungskräften der Zambia Consolidated Copper Mines (ZCCM) - ergaben, daß die bei der ersten Antragstellung auf Sysmin-Darlehen auftretenden Verzögerungen bei der zweiten Antragstellung unter Lomé III bereits nicht mehr in dem Maße auftraten wie un-

37 Vgl. Weltbank (Hrsg.): Price prospects for major primary commodities, Vol. IV: Metals and Minerals, Washington, D.C., Oktober 1986, S. 25; O.V.: Copper, Short-term shine, a.a.O., S. 68 f.

38 Vgl. O.V.: Chiles Produktionskosten ..., a.a.O.

39 Persönliches Gespräch, Norddeutsche Affinerie, Hamburg.

40 ab Produktionsstätte bis europäischer Hafen, vgl. Zambia Mining Yearbook, 1980, a.a.O., S. 30.

41 Vgl. Zambia Consolidated Copper Mines (Hrsg.): Annual Report 1988, S. 4 f. 
ter Lomé Il. Beide Seiten - Antragsteller und die EG-Kommission - hatten Erfahrungen gesammelt und umsetzen können.

In diesem Zusammenhang ist anzumerken, daß im nachfolgenden dargestellte Mängel des Verfahrens nicht für Fehler und Versäumnisse der Bergbaugesellschaften verantwortlich gemacht werden sollen. Vielmehr ist es Zweck der Kritik, weitere Möglichkeiten zur Optimierung der SysminRegelung, die sich erst im praktischen Umgang mit dem Verfahren herausstellen, aufzuzeigen.

Seit Mitte der siebziger Jahre zeichnete es sich immer deutlicher $a b$, daß die selbstfinanzierten Mittel der ZCCM beziehungsweise ihrer Vorgängergesellschaften nicht mehr zur Aufrechterhaltung der Produktionskapazitäten ausreichen würden. Aufgrund zunehmender Schwierigkeiten bei der Produktion, einer abnehmenden Nachfrage und eines ab dem Jahre 1981 fallenden Kupferpreises ${ }^{42}$ sah sich das Unternehmen nicht mehr in der Lage, die erforderlichen Investitionen und Anschaffungen, die nahezu ausschließlich aus dem Ausland bezogen wurden, zu tätigen.

In den ersten 10 Jahren der Unabhängigkeit (1964 - 1974) wurden durch den Export von Kupfer und Kobalt genügend Devisen erwirtschaftet, so da $B$ die vorgeschriebenen 75 Prozent der Deviseneinnahmen ohne Schwierigkeiten beim Staat gegen die Landeswährung Kwacha eingetauscht werden konnten und dem Unternehmen dennoch ausreichend Devisen verblieben, um z.B. Ersatzteile aus dem Ausland zu beziehen. Doch ab dem Jahre 1976 ging die Kupferproduktion Sambias zurück, allein von $715.000 \mathrm{t}$ im Jahre 1976 auf $590.000 \mathrm{t}$ im Jahre $1981 .{ }^{43}$

Der damit einhergehende Rückgang der Exporterlöse, der durch einen drastischen Preisverfall ${ }^{\text {t4 }}$ verstärkt wurde, wirkte sich in zweifacher Weise auf die sambische Volkswirtschaft aus: Sowohl der Staat, der etwa 95 Prozent seiner Devisenerlöse ${ }^{45}$ aus dem Bergbau bezieht, hatte Einnah-

42 S. Abb. 9a; Vgl. Metallgesellschaft (Hrsg.): Metallstatistik 1975/1985, a.a.O., S. 415

$43 \mathrm{Vgl}$. Pagni, L.: Getting out of the copper straitjacket, a.a.O., S. 19.

4 Allein von 1980 bis 1981 fiel der Kupferpreis um 18,7 \%. Vgl. Statistisches Amt der EG (Hrsg.): EC-ACP-Trade, a.a.O., S. 404, Tabelle 4.2a.

45 Vgl. Zambia Consolidated Copper Mines (Hrsg.): Annual Report 1983, a.a.O., S. 4. 
meeinbußen hinzunehmen als auch die ZCCM, die sich nicht mehr in der Lage sah, ihre Aufwendungen zu tragen. Vor allem reichten die 25 Prozent der Exporterlöse, die der ZCCM in Form von Devisen erhalten blieben, nicht mehr aus, um notwendige Bergbauausrüstungen aus dem Ausland, im wesentlichen aus den westlichen Industriestaaten, zu importieren.

Ohne externe finanzielle Unterstützung (prognostizierter Gesamtbedarf für das Anfang der achtziger Jahre begonnene, erste Rehabilitationsprogramm etwa 300 Mio. US-\$46) konnte die Produktion nicht im erforderlichen Maße aufrechterhaiten werden.

Der Teufelskreis über geringere Einnahmen zu weiteren Produktionseinschränkungen war nur mit Hilfe ausländischen Kapitals zu durchbrechen, eine Reduzierung der ZCCM-Abgaben an den Staat war zu dieser Zeit noch nicht durchsetzbar. Aufgrund dieser Erkenntnis überprüfte das ZCCM-Management sämtliche Möglichkeiten, um ausländisches Kapital zur Unterstützung heranzuziehen. Neben Lieferanten konsultierte man die Weltbank (IBRD), die Afrikanische Entwicklungsbank (ADB) und wegen der Lomé-Verträge die Europäische Gemeinschaft.

Bis zum Jahre 1988 erhielt die ZCCM Darlehen in Höhe von 75 Mio. US-\$ von der Weltbank, 26 Mio. US-\$ von der Afrikanischen Entwicklungsbank, über $30 \mathrm{Mio}$. ECU von der EIB,47 4,5 Mio. ECU aus Italien (dieses Darlehen wird von der EG-Kommission verwaltet) sowie zwei Tranchen aus der Sysmin-Fazilität (s. Kap. 4.2: 55 Mio. ECU und 28 Mio. ECU unter Lomé II). Eine dritte Sysmin-Tranche wurde im Jahre 1987 beantragt; die Entscheidung über die Gewährung und Höhe dieses Darlehens wird jedoch von dem Resultat einer Evaluierung der ZCCM abhängig gemacht. Zu diesem Zwecke haben im März 1989 europäische

46 Persönliches Gespräch, ZCCM, Lusaka, Sambia; Vgl. Delegation der Kommission der Europäischen Gemeinschaften (Hrsg.): Co-operation Zambia ..., a.a.O., S. 22.

47 Sambia erhielt bis zum Jahre 1987 insgesamt 57,6 Mio. ECU von der EIB, davon 46,5 Mio. ECU aus eigenen Mitteln der Bank und Haushaltsmitteln der Gemeinschaft u.a. für Vorhaben im Bergbau; Vgl. Europäische Investitionsbank (Hrsg.): Jahresbericht 1987, Luxemburg 1988, S. 74 und S. 11. 
Bergbauberater (französische Bergbau-Consultants (ERNA)) ihre Arbeit im sambischen Kupfergebiet aufgenommen. ${ }^{48}$

Da Sambia als erster AKP-Staat bei der EG-Kommission in Brüssel einen Antrag auf Darlehen aus der Sysmin-Fazilität stellte, war nicht zu erwarten, daß diese Angelegenheit als Routinefall behandelt werden würde.

Nach mehrmonatiger Wartezeit und Besuchen von Delegationen, sowohl in Brüssel als auch in Sambia, wurde der erste Sysmin-Antrag genehmigt. ${ }^{49}$ Von dem Entschluß der Unternehmensführung den Antrag zu stellen bis zur Genehmigung im Jahre 1982 verging über ein Jahr. Die Genehmigung des zweiten ZCCM-Antrages im Oktober 1985 dagegen erfolgte zügig, denn sowohl die antragstellenden Behörden Sambias und das Unternehmen als auch die EG-Kommission waren mit dem erforderlichen Prozedere beziehungsweise der Situation der Abbaubetriebe vertraut. Es darf dabei jedoch nicht übersehen werden, daß die ersten Vorbereitungen für die zweite Antragstellung in den Abbaubetrieben bereits im Sommer 1984 begonnen wurden. ${ }^{50}$

Die leicht begrenzte, internationale Ausschreibung (s. Kap. 2.5.3) erfordert in der Regel ebenfalls eine Zeitspanne von bis zu mehreren Monaten, da mit dieser Vorgehensweise zum Teil zeitintensive Rückfragen bei der EG-Kommission verbunden sind, z.B. dann, wenn die eingebenden Angebote nicht exakt den Spezifikationen entsprechen.

Bis zum August 1984 waren immer noch nicht alle der über den ersten Sysmin-Antrag ausgeschriebenen Bergbauausrüstungen in den einzelnen Abbaubetrieben der ZCCM eingetroffen. Zwar handelte es sich dabei im wesentlichen um Sonderanfertigungen, dringend benötigt wurden diese Bergbauausrüstungen jedoch auch.

Es gab jedoch auch Fälle, in denen prompt und unbürokratisch reagiert wurde, beispielsweise konnte ein von der ZCCM - schon vor der Genehmigung des Sysmin-Antrags - abgeschlossener Kaufvertrag über die Liefe-

48 Persönliche Gespräche, Beratender Ingenieur der ZCCM, Lusaka, Sambia; Kommission der EG, Brüssel; Vgl. Delegation der Kommission der Europäischen Gemeinschaften (Hrsg.): Co-operation Zambia ..., a.a.O., S. 15.

49 Persönliches Gespräch, ZCCM-Headoffice, Lusaka, Sambia.

so Persönliche Gespräche, Nchanga und Mufulira, ZCCM, Sambia 1984. 
rung von Kompressoren aus Mitteln der Sysmin-Fazilität beglichen werden. ${ }^{51}$

Ende November 1987 waren 2,5 Mio. ECU (5 Prozent) der ersten Sysmin-Tranche und 11,7 Mio. ECU (42 Prozent) der zweiten Sysmin-Tranche noch nicht gezogen worden. ${ }^{52}$

Die Konditionen der Sysmin-Darlehen, die über den sambischen Staat an die ZCCM weitergeleitet werden, sind für Sambia extrem günstig: Laufzeit 40 Jahre, davon 10 Jahre tilgungsfrei. Als Zuschüsse werden die Mittel erst seit Lomé IV vergeben. Sambia zahlt für die Darlehen 1 Prozent Zinsen p.a.; der Zinssatz, den die ZCCM abzuführen hat, beträgt 6 Prozent.

Die Zinsdifferenz wird an einen staatlich verwalteten Sozialfonds abgeführt. Mittel aus diesem Fonds müssen - so die Auflage der EG-Kommission - für soziale Einrichtungen in den ZCCM-Abbaubetrieben, wie Kindergärten, Schulen, Krankenhäuser, eingesetzt werden. ${ }^{53}$

Wie Gespräche mit den für die Sysmin-Anträge verantwortlichen ZCCMManagern ergaben, würden im Zusammenhang mit dem Sysmin-Verfahren folgende Modifikationen begrüßt: Zunächst sollte die Einschaltung von Behörden und Institutionen des antragstellenden AKP-Staates auf ein Minimum reduziert werden. Es stellte sich in Sambia heraus, daß der Prozeß der Antragstellung durch staatliche Inkompetenz, mangelndes Engagement sowie eine übermäßige Bürokratie bei den antragstellenden Staatsorganen zusätzlich verzögert wurde. ${ }^{54}$

Ein weiterer Änderungswunsch basiert auf der Tatsache, daß fïhrende Anbieter von Bergbauausrüstungen ihre Produktionsstätten nicht in den Unterzeichnerstaaten der Lome-Abkommen betreiben. Somit können Angebote aus den USA, Kanada, der Republik Südafrika, Japan etc. - auf-

51 Persönliches Gespräch, ZCCM-Mufulira, 1984.

52 Persönliches Gespräch, ZCCM, Lusaka, Sambia; Vgl. auch Rechnungshof der EG (Hrsg.): Jahresbericht zurn Haushaltsjahr 1985, a.a.O., S. 145.

53 Persönliches Gespräch, EG-Delegation, Lusaka, Sambia; Vgl. Delegation der Kommission der Europäischen Gemeinschaften (Hrsg.): Cooperation Zambia ..., a.a.O., S. 15.

54 Persönliches Gespräch, ZCCM-Headoffice, Lusaka, Sambia. 
grund der primär auf die Unterzeichnerstaaten begrenzten Ausschreibung (s. Kap. 2.5.3) - nicht berücksichtigt werden.

Obwohl das Bemühen der EG-Kommission, Aufträge für die EG-Industrien heranzuholen, verständlich ist, muB diese Regelung für die begünstigten Gesellschaften nicht immer sinnvoll sein. Dies trifft insbesondere $z u$, wenn Bergbauausrüstungen zu beschaffen sind, die zu den bereits eingesetzten Geräten nicht kompatibel sind, da diese aus einer anderen, auBereuropäischen Bezugsquelle stammen. Wie die Erhebungen in Sambia zeigten, sind dann neue Ersatzteillager zu erstellen und Reparaturanlagen umzurüsten; außerdem muß das Personal neu eingewiesen werden.

Dieser unerwünschte Denormierungseffekt kann, wie im Falle Sambias, auch eintreten, wenn das von den Bergbauunternehmen bereits genutzte Gerät aus der Europäischen Gemeinschaft bezogen wurde, die EG-Kommission aber darauf besteht, nur das billigste Angebot zu akzeptieren. Auch diese Bedingung kann bei der notwendigen Durchführung der oben aufgeführten Begleitmaßnahmen für den Darlehensempfänger recht kostenintensiv werden.

In einem Fall - so wurde in einem ZCCM-Abbaubetrieb berichtet - handelte es sich um Transportfahrzeuge, wobei der Hersteller Fiat den $\mathrm{Zu}-$ schlag erhielt, obwohl das Unternehmen Daimler Benz mit dem zweitgünstigsten Angebot schon seit vielen Jahren Lieferant der ZCCM war. ${ }^{55}$

Generell jedoch sind Ausschreibungen durchaus vorteilhaft, da es häufig gelingt, existierende Liefermonopole zu brechen. Bei plötzlich auftretender Konkurrenz lassen sich überhöhte Händlerpreise nicht länger aufrechterhalten. ${ }^{56}$

Anlaß zur Kritik liefert auch das Bestreben der EG-Kommission, mit Hilfe der Sysmin-Mittel möglichst umfangreiche Investitionsgüterlieferungen zu initiieren. Natürlich sind die Europäer interessiert, für einen Großteil der günstig finanzierten Kredite Ausrüstungen in das Empfängerland zu verkaufen. Dennoch sollte im Rahmen eines Wiederherstellungsprogrammes - im Gegensatz zu einem Aufbauprojekt - primär versucht werden, die schon vorhandenen Kapazitäten wieder einsatzfähig zu machen. 
Dieses Ziel darf aber auch nicht durch reine Ersatzteillieferungen angestrebt werden, denn es würde - wie die empirischen Untersuchungen in Sambia deutlich machten - schon nach kurzer Zeit erneut Darlehen für weitere Ersatz- und Verbrauchsgüter notwendig.

Insofern scheint eine ausgewogene Zusammensetzung der Maßnahmen, das heißt sowohl Wiederherstellung der Einsatzfähigkeit von bereits existierenden Bergbauausrüstungen als auch Ersatz, Erneuerung und Ergänzung, für das betroffene Bergbauunternehmen am sinnvollsten.

Bei der Neubeschaffung wäre dafür Sorge zu tragen, daß die Betriebsbereitschaft auch über längere Zeiträume, zum Beispiel durch über das in Europa übliche Maß hinausgehende Wartungsverträge, gewährlejstet ist. Damit würde verhindert, daß - wie bei der ZCCM geschehen - ein aus Europa importiertes, hochtechnisiertes Gerät bereits wenige Wochen nach der Lieferung unbrauchbar wird, weil ein essentielles Ersatzteil fehlt. ${ }^{57}$

\subsubsection{Fallbeispiel Simbabwe}

Die Republik Simbabwe erstreckt sich über eine Fläche von etwa 390.000 $\mathrm{km}^{2}$ und ist damit halb so groß wie das nordwestliche Nachbarland Sambia. Gemeinsame Grenzen besitzt Simbabwe weiterhin mit Mosambik, Südafrika, Botswana und Namibia.

Wie im Falle Sambias besitzt der im südlichen Zentralafrika gelegene Staat keine eigenen Küsten und muß daher Überseetransporte, soweit sie nicht mit Flugzeugen zu bewerkstelligen sind, ebenfalls mit Unterstuitzung der Nachbarn abwickeln (s. Karte 2).

Das tropische Klima wird aufgrund der durchschnittlichen Höhenlage des Landes von 400 bis $1800 \mathrm{~m}$ gemildert.

Die Bevölkerung Simbabwes wird auf etwa 9 Mio. Einwohner geschätzt und ist damit größer als die Sambias. Die Anzahl der Weißen soll mittlerweile auf 100.000 Personen zurückgegangen sein. Wie in vielen Entwicklungsländern weist die Bevölkerungspyramide Simbabwes eine breite

57 Persönliches Gespräch, Nchanga, ZCCM, Sambia. 
Karte 2: Republik Simbabwe

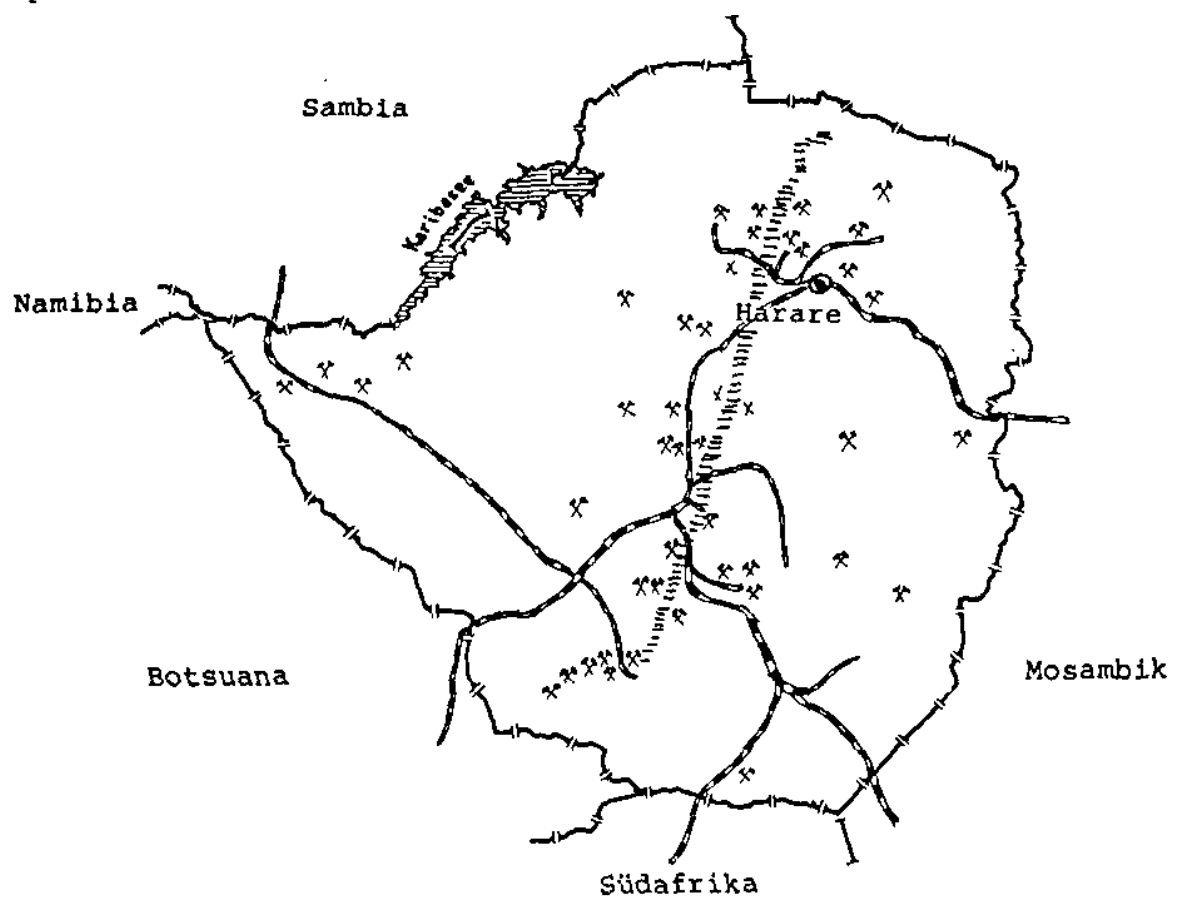

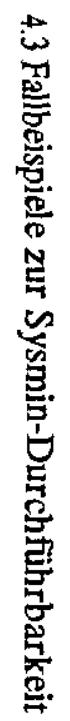

Legende

- Greal Dyke

Bargbau

- Eisenbohn

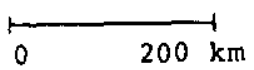

Quelle: Statistisches Bundesamt Wiesbaden (Hrsg.): Länderbericht Simbabwe, Stuttgart 1985, S. 8. 
Basis auf, d.h. der Anteil der Jugendlichen an der Gesamtbevölkerung ist sehr hoch; über 70 Prozent der Simbabwer sind jünger als $25 \mathrm{Jahre.}{ }^{58} \mathrm{Die}$ Probleme, die aus der Landflucht erwachsen, ähneln denen Sambias; nach Schätzungen der Vereinten Nationen wächst die Bevölkerung in den Städten jährlich um 7 Prozent an. ${ }^{99}$ Die damit verbundene Arbeitslosigkeit (1988: ca. 1 Mio. Arbeitslose bei etwa 3 Mio. Erwerbsfähigen) wird als das größte Einzelproblem der Wirtschaft Simbabwes angesehen. ${ }^{\infty}$

Nach einem Bürgerkrieg wurde die britische Kolonie (Süd-)Rhodesien am 18. April 1980 unabhängig. Der Nachfolger des weißen Ministerpräsjdenten Smith wurde Mugabe, der bis heute Regierungschef ist.

Wie Sambia ist Simbabwe Mitglied bedeutender Organisationen, wie der Vereinten Nationen, des Internationalen Währungsfonds, der Organisation für Afrikanische Einheit. Seit seiner Unabhängigkeit ist es über die LomeAbkommen mit der Europäischen Gemeinschaft assoziiert.

Simbabwe besitzt eine im starken Maße diversifizierte, weiterverarbeitende Industrie (s. Tab. 18); das Land gehört zu den am stärksten industrialisierten Staaten Afrikas.

Dies resultiert zum einen aus der Konzeption der britischen $\mathrm{K}_{0}$ lonialverwaltung, wonach die Kupfererze im Norden Rhodesiens (Sambia) abgebaut und dann im südlichen Teil Rhodesiens (Simbabwe) weiterverarbeitet worden sind. ${ }^{61}$

Zum anderen wurde ab 1965 der Ausbau des weiterverarbeitenden Gewerbes forciert, da im Zusammenhang mit den Unabhängigkeitsbestrebungen stehende Boykottmaßnahmen Importsubventionen nötig machten. ${ }^{62}$

58 Vgl. Statistisches Bundesamt Wiesbaden (Hrsg.): Länderbericht Simbabwe, Stuttgart 1985, S. 17 f.; Bundesstelle für Außenhandelsinformation (Hrsg.): Wirtschaftsdaten aktuell, Simbabwe, Oktober 1988.

59 Vgl. Statistisches Bundesamt Wiesbaden (Hrsg.): Länderbericht Simbabwe, a.a.O., S. 20.

60 Vgl. O.V.: Aufschwung auf schwacher Basis in Simbabwe, in: Neue Zürcher Zeitung, 7. Dezember 1988.

6) Persönliches Gespräch, Bergbauministerium von Sambia, Lusaka.

62 O.V.: Rebuilding Zimbabwe, ZANU, Harare (1985), S. 55. 
Neben der Metallverarbeitung sind die Nahrungsmittel-, Textil-, Möbel-, Elektro-, Tabak- und Ledererzeugung die dominierenden Industriezweige. Diese für afrikanische Verhältnisse fortgeschrittene Industrialisierung erklärt unter anderem den - im Vergleich zu Sambia - relativ geringen Beitrag des Bergbaues zum Bruttoinlandsprodukt Simbabwes (s. Tab. 17 und Anhang 2).

Der Bergbau erwirtschaftet bei einer Exportquote von 80 Prozent etwa 50 Prozent der Devisenerlöse, beschäftigt aber nur rund 6 Prozent der Erwerbsbevölkerung. ${ }^{63}$

In Gegensatz zu Sambia oder Zaire ist die Rohstoffproduktion Simbabwes nicht auf wenige Erzeugnisse beschränkt. Über 70 verschiedene Bergbauprodukte werden hauptsächlich entlang des von Norden nach Süden verlaufenden Great-Dyke-Höhenrückens abgebaut (s. Karte 2). Rund 85 Prozent der Erlöse für Bergbauerzeugnisse allerdings werden durch die 6 Rohstoffe Gold (30 Prozent), Asbest, Nickel, Kohle, Kupfer und Chrom erzielt $^{\text {t4 }}$ (s. Tab. 21).

Eine besondere Bedeutung kommt den Asbest- und Chromitvorkommen zu, da Simbabwe viertgrößter Asbestproduzent ist und mit der Republik Südafrika zusammen 95 Prozent der Weltvorräte an Chromit besitzt. ${ }^{65}$

Im Jahre 1987 wurde bei vielen mineralischen Rohstoffen ein Produktionsanstieg verzeichnet, der sich im Jahre 1988 fortsetzte. Dennoch wurden die Förderraten aus der Zeit vor der Unabhängigkeit im Jahre 1980 nur bei Gold und Kohle überschritten. ${ }^{66}$ Nach einer Rekord-Tabak-

63 Vgl. O.V.: Rebuilding Zimbabwe ..., a.a.O., S. 49 und S. 52.

$64 \mathrm{Vgl}$. Statistisches Bundesamt Stuttgart (Hrsg.): Länderbericht Simbabwe 1985, a.a.O., S. 43 f.

65 Vgl. Schmidt, H; Kruszona, M: Regionale Verteilung ..., a.a:O., S. 69 und S. 110; Harms, U.; Iwersen, A.: Rohstoffgewinnung in Entwicklungsländern, a.a.O., S. 88; Gocht, W. (Hrsg.): Handbuch der Metallmärkte, a.a.O., S. 89 f.

of $\mathrm{Vgl}$. Bundesstelle für Außenhandelsinformation (Hrsg.): Wirtschaftslage, Simbabwe zur Jahresmitte 1988, August 1988, S. 2; O.V.: Simbabwe: Der Bergbau mit einem besseren Ergebnis, in: Blick durch die Wirtschaft, 9. Mai 1988. 
ernte im Jahre 1988 wurde Gold zunächst wieder als wichtigste Devisenquelle abgelöst. ${ }^{67}$

In Zukunft soll auch der Produktion von Silizium, Kobalt und Platin verstärkte Aufmerksamkeit gewidmet werden, da für diese Erzeugnisse eine steigende Nachfrage zu erwarten ist. Durch den Einsatz neuer Gewinnungstechniken und die Inbetriebnahme neuer Bergwerke Anfang des Jahres 1990 erwartet man eine starke Zunahme der Goldproduktion. In 5 bis 10 Jahren wird eine Verdoppelung der Fördermenge durchaus für realistisch gehalten. ${ }^{68}$

Tabelle 21: Die Bergbauproduktion Simbabwes

\begin{tabular}{|ll|r|r|r|r|}
\hline & & 1980 & 1981 & 1982 & 1983 \\
\hline Gold* & $(\mathrm{t})$ & 11 & 12 & 13 & 14 \\
Asbest & $(1.000 \mathrm{t})$ & 251 & 248 & 194 & 153 \\
Nickel* $(1.000 \mathrm{t})$ & 15 & 13 & 13 & 10 \\
Kohle & $(1.000 \mathrm{t})$ & 3.134 & 2.867 & 2.769 & 3.326 \\
Kupfer* $(1.000 \mathrm{t})$ & 27 & 25 & 25 & 21 \\
Chrom* (1.000 t) & 554 & 536 & 432 & 431 \\
Kobalt* (t) & 115 & 94 & 98 & 73 \\
Zinn* & $(\mathrm{t})$ & 934 & 1.157 & 1.197 & 1.235 \\
\hline
\end{tabular}

Anm.: *) Metallinhalt.

Quelle: Eigene Zusammenstellung nach: O.V.: Rebuilding Zimbabwe, ZANU, Harare (1985), S. 51 und S. 53.

Während in Sambia alle bedeutenden Kupferbergwerke zur überwiegend im staatlichen Eigentum befindlichen ZCCM gehören, befinden sich die

67 Vgl. O.V.: Aufschwung auf schwacher Basis in Simbabwe, in: Neue Zürcher Zeitung, 7. Dezember 1988.

$68 \mathrm{Vgl}$. Bundesstelle für Außenhandelsinformation (Hrsg.): Wirtschaftslage, Simbabwe zur Jahresmitte 1988, a.a.O., S. 4; 0.V.: Simbabwe will in den Bergbau investieren, in: Blick durch die Wirtschaft, 12. Februar 1990. 
Abbaubetriebe Simbabwes bis auf wenige Ausnahmen in privater Hand. Die Eigentümer sind häufig transnationale Bergbaugesellschaften, wie die Rio Tinto Gruppe, Union Carbide und Anglo American. ${ }^{69}$

Beschäftigungspolitisch spielt der in Simbabwe noch hãufig anzutreffende Kleinbergbau nur eine untergeordnete Rolle. Obwohl im Jahre 1981 von 203 Bergwerken 110 Betriebe weniger als 50 Mitarbeiter einsetzten, waten in ihnen nur 3 Prozent der etwa 60.000 Beschäftigten des Bergbauseltors tätig. Die 67 Bergwerke mit mehr als 50 bis zu 500 Bergleuten beschäftigten 14 Prozent der im Bergbau tätigen Simbabwer. ${ }^{70}$

Im Jahre 1982 mußte eine große Zahl kleinerer Bergbauunternehmen schließen, da durch die weltweit niedrigen Rohstoffpreise ihre Rentabilität nicht mehr gewährleistet war.

Mit Hilfe staatlicher Mittel gelang es jedoch, das Einstellen der Produktion in den größeren Bergwerken zu verhindern. ${ }^{71}$ Die Zahl der Beschäftigten ist nach einem Rückgang von 13 Prozent im Jahre 1983 wieder auf etwa 55.000 im Jahre 1986 angestiegen. ${ }^{72}$ Damit arbeiten in der sambischen ZCCM mehr Personen als im gesamten Bergbausektor Simbabwes.

Besonders deutlich macht sich im Bergbau Simbabwes das Fehlen qualifizierter Facharbeiter bemerkbar. ${ }^{73}$ Der Mangel resultiert zum größten Teil aus dem Abwandern von Bergleuten europäischer Abstammung, welche nach dem Unabhängigkeitskrieg in Simbabwe keine Existenzmöglichkeit mehr sahen. $\mathrm{Zu}$ dem Facharbeitermangel kommt erschwerend hinzu, daß bei den eingesetzten Bergbauausrüstungen ein erheblicher Modernisierungsstau besteht. Zwar wurden zur Reduktion dieser Schwierigkeiten unterstützende Maßnahmen durch die Regierung eingeleitet, trotzdem werden in naher Zukunft auf die einzelnen Bergbauunternehmen noch erhebliche Investitionen zukommen. ${ }^{74}$ Prinzipiell werden die Investitionen durch die staatliche Devisenbewirtschaftung erschwert, die gesamte Wirt-

69 Vgl. Mining Annual Review 1985, S. $426 \mathrm{f}$.

70 Vgl. Statistisches Bundesamt Stuttgart (Hrsg.): Länderbericht Simbabwe 1985, a.a.O., S. 44.

7 Vgl. 0.V: Rebuilding Zimbabwe ..., .a.a.O., S. 45.

72 Vgl. Mining Annual Review 1984, S. 385; Derselbe 1987, S. 403.

73 Ebenda, S. 403.

74 Vgl. Mining Annual Review 1986, S. 403; O.V.: Rebuilding Zimbabwe ..., a.a.O., S. 45 ff. 
schaft des Landes hat darunter zu leiden. Nach Abschätzungen könnte der Bergbausektor seine Produktion um bis zu fünf Prozent steigern, wenn genügend Devisen allein zum Kauf von Ersatzteilen vorhanden wären. Angabegemäß erhält der Bergbausektor nur 50 Prozent seines Bedarfs an Ersatzteilen, Ausrüstungen und Maschinen. ${ }^{75}$

Im Jahre 1988 konnte der Bergbausektor zum ersten Male seit 3 Jahen wieder eine zunehmende Produktion und angestiegene Erlöse verzeichnen, im wesentlichen bedingt duch die deutlich angestiegenen Nachfragen nach Bergbauerzeugnissen, neu angewandte Bergbautechniken sowie die $\mathrm{Er}$ schließung weiterer Lagerstätten. ${ }^{76}$

Sollte bei einem der durch die Sysmin-Regelung erfaßten Produkte die Auslöseschwelle erreicht werden, könnte Simbabwe beziehungsweise die betroffenen Bergbauunternehmen durchaus Mittel aus dem Sysmin-Fonds erhalten. Angesichts der Struktur der Bergbauindustrie in Simbabwe, die im wesentlichen aus kleineren und mittleren Unternehmen besteht, ist jedoch zu erwarten, daß die Möglichkeiten der Sysmin-Faziliät nicht in Anspruch genommen werden.

\subsection{Die Möglichkeiten der Sysmin-Regelung zur Schaffung von Ver- knüpfungs- und Sekundäreffekten}

In den ersten Jahren nach Inkrafttreten wurde den Lomé-Abkommen gelegentlich unterstellt, daß bestehende Handelsstrukturen aufgrund der Vereinbarungen verfestigt sowie die Etablierung einer weiterverarbeitenden Industrie verhindert würden. Die Bildung von Verknüpfungs- aber auch von Sekundäreffekten beziehungsweise deren gezielte Förderung erschien den Kritikern nicht weitgehend genug berücksichtigt zu sein.

In der Tat beschränken sich wichtige Regelungen in den Lome-Abkommen, wie das Sysmin-Verfahren oder das Stabex-Verfahren, in ihren

$75 \mathrm{Vgl}$ O.V.: Aufschwung auf schwacher Basis in Simbabwe, a.a.O.; O.V.: Simbabwe will den Bergbau intensivieren, a.a.O.

$76 \mathrm{Vgl}$. Bundesstelle für Außenhandelsinformation (Hrsg.): Wirtschaftslage, Simbabwe ..., a.a.O., S. 1 und S. 3 f.; O.V.: Aufschwung auf schwacher Basis in Simbabwe, a.a.O.; O.V.: Simbabwe will den Bergbau intensivieren, a.a.O. 
Bemessungsgrundlagen auf Rohstoffe oder Erzeugnisse der ersten Verarbeitungsstufe. ${ }^{77}$ Schwankungen von Exporterlösen beispielsweise, die sich aus dem Absatz von Halb- und Fertigprodukten ergeben, werden in dem Sysmin-Verfahren nicht berücksichtigt.

In der Havanna-Charta (1948) ist ein Rohstoff definiert als "any product of farm, forest or fishery or any mineral, in its natured form or which has undergone such processing as is costumarily required to prepare it for marketing in substantial volume in international trade" ${ }^{78}$ Die Definition für mineralische Rohstoffe knüpft daran an und umfaßt "alle festen, flüssigen und gasförmigen Minerale und Mineralgemische, die in bergbaulichen Betrieben, Fördereinrichtungen oder Hüttenwerken gewonnen wurden". 79

Das bei der Errichtung der Sysmin-Regelung kritisierte Nichterfassen von Hablb- und Fertigprodukten in der Abhängigkeits- und Auslöseschwelle läßt sich durch folgende Argumente begründen:

- Bis heute exportieren die AKP-Staaten im wesentlichen Roh- und Grundstoffe, so daß ein Berücksichtigen von Produkten höherer Verarbeitungsstufen vorläufig nur unwesentliche Verbesserungen im Hinblick auf die Vergabe von Mitteln aus der Sysmin-Fazilität bringen würde.

- Die Abhängigkeit einer Volkswirtschaft von dem Export einzelner Halb- oder Fertigwaren ist aufgrund einer größeren Produktionsbreite geringer.

- In der Regel unterliegen Halb- und Fertigprodukte im Gegensatz zu Roh- und Grundstoffen weniger starken Preisschwankungen, da diese Erzeugnisse unter anderem höhere Nachfrageelastizitäten aufweisen.

77 Zum Beispiel ist Aluminium nicht in dem Sysmin-Katalog erfaßt, wohl aber Bauxit und Aluminium (Lome IV: Art. 215 Abs. 1a).

$78 \mathrm{Vgl}$. Art. 56, Abs. 1 der Havanna-Charta, in: Krappel, F.: Die Havanna Charta und die Entwicklung des Weltrohstoffhandels, Berlin 1975, S. 109.

79 Vgl. Gocht, W.: Wirtschaftsgeologie und Rohstoffpolitik, a.a.O., S. 13. 
- Da die Vergleichbarkeit von Erzeugnissen höherer Verarbeitungsstufen nicht immer möglich ist, würden die Genehmigungsverfahren komplizierter und die Entscheidungsprozesse langwieriger werden.

Eine Beschränkung der Sysmin- beziehungsweise auch der Stabex-Regelung auf die oben definierten Verarbeitungsstufen erscheint somit den auch in den Lomé-Abkommen - angestrebten entwicklungspolitischen Grundsatz, die industrielle Weiterverarbeitung in den Entwicklungsländern zu begünstigen (Lomé IV: Art. 17), unberücksichtigt zu lassen. Die anfänglich geäußerte Sorge, daß sich die "Monokulturen" mit ihrer einseitigen Produktionsstruktur und extremen Exportabhängigkeit stabilisieren könnten, ${ }^{80}$ und daß so die AKP-Länder verleitet würden, die nicht erfaßten Rohstoffe zu vernachlässigen, ${ }^{81}$ ist bei einer oberflächlichen Betrachtung nicht ganz unbegründet.

Bei einer detaillierten Analyse des gesamten Komplexes der Lomé-Abkommen erweist sich diese Vermutung jedoch als unzutreffend. Generell steht den AKP-Staaten eine Vielzahl von unterstützenden Maßnahmen bei der Etablierung von weiterverarbeitenden Unternehmen zur Verfügung, deren Umfang und Zielsetzungen die Möglichkeiten der Sysmin-Regelung aber auch des Stabex-Verfahrens weit übertreffen.

Diese Instrumente wurden bei den Verhandlungen zu Lomé IV wesentlich erweitert sowie ausgebaut. Neben den bereits in Lomé III etablierten Maßnahmen, zum Beispiel zur Erschließung des Bergbau- und Energiepotentiales oder der Bereitstellung von Mitteln der Europäischen Investitonsbank bei der Bildung von Joint Ventures, steht nun auch Risikokapital beziehungsweise Kapital der Europäischen Investitionsbank für Zwecke zur Verfügung wie Diversifikation, Unterstützung von Umweltschutzmaßnahmen, Aufbau von Infrastrukturen, Förderung der regionalen Kooperation (Lomé IV: Art. $101 \mathrm{ff}$.). Ein in Lomé IV neu etabliertes Rohstoffkomitee soll die Aktivitäten im Rohstoffsektor der AKP-Staaten koordinieren, fördern und überwachen (Lome IV: Art. 75) und somit dazu beitragen, daß die oben erwähnte Bildung von einseitigen Produktionsstrukturen nicht weiter gefördert, sondern sogar reduziert wird.

80 Vgl. Gerdes, U.; Bosse, P.: Stabex kontra Rohstoff-Fonds, in: epd Dritte-Welt-Information 10/75, aktualisierte Fassung 1979, S. 3.

81 Vgl. Helldorf, K.v.: Die Abkommen von Lomé, a.a.O., S. 10. 
Bei der Diskussion der Verknüpfungs- beziehungsweise Sekundäreffekte und der in diesem Zusammenhang geäußerten Kritik an dem Sysmin-Verfahren, es würde diese zu wenig fördern, muß differenziert werden:

Zum einen gibt es die sogenannten vorwärtigen und rückwärtigen Verknüpfungseffekte ("Linkages"), die als Basis für eine allgemeine industrielle Entwicklung dienen können und im Rahmen eines globalen entwicklungspolitischen Konzeptes gesehen werden müssen. Zum anderen treten bei der Realisierung und dem Betrieb großer Projekte begleitende, primär nicht beabsichtigte Effekte auf, die sich in der Regel positiv auf die Entwicklung einer Region oder eines Landes auswirken, sogenannte Sekundäreffekte. ${ }^{82}$

In der Regel wird davon ausgegangen, daß bei den Verknüpfungseffekten die sogenannten "backward-linkages" in stärkerem Maße eintreten als die "forward-linkages", weshalb von Entwicklungstheoretikern häufig empfohlen wird, in Entwicklungsländern zunächst mit dem Aufbau von weiterverarbeitenden Industrien $\mathrm{zu}$ beginnen. ${ }^{83}$

Diese Forderung ist in Bergbauländern nicht leicht zu realisieren, da ein Großteil der Entwicklungsprogramme auf die Optimierung und Wiederherstellung der schon bestehenden, extraktiven Industrien - die am Anfang des Produktionsprozesses stehen - ausgerichtet ist.

Abhängig sind solche Verknüpfungseffekte von dem gewonnenen Rohstoff, von der Lage der Projekte und vom Entwicklungsstand der Länder.

Nur geringe Verknüpfungseffekte beispielsweise werden der Zinnproduktion nachgesagt, ${ }^{84}$ obwohl die Erzeugung von Primär-Feinzinn in den westlich orientierten Staaten zu etwa 80 Prozent in den Produzenten-

82 Vgl. Hemmer, H.R.: Wirtschaftsprobleme der Entwicklungsländer, a.a.O., S. 450; Gocht, W.R., Zantop, H.; Eggert, R.G.: International Mineral Economics, a.a.O., S. 247 f.

$83 \mathrm{Vgl}$. Hemmer, H.R.: Wirtschaftsprobleme der Entwicklungsländer, a.a.O., S. $452 \mathrm{f}$.

4 Vgl. Harms, U.; Iwersen, A.: Rohstoffgewinnung in Entwicklungsländern, a.a.O, S. $202 \mathrm{ff}$. 
ländern erfolgt. ${ }^{85}$ Das heißt, daß sich diese Effekte nicht automatisch einstellen müssen, selbst wenn - wie in vielen Entwicklungsländern - bereits auf dem Produktionsniveau der ersten Verarbeitungsstufen gearbeitet wird.

Im Gegensatz zur Zinn- aber auch der Kobaltgewinnung werden die Verknüpfungseffekte zu den vor- und nachgelagerten Bereichen bei der Kupfer- und Bauxit-Aluminium-Produktion als intensiv bezeichnet, ${ }^{86}$ obwohl die jeweiligen Weiterverarbeitungsstufen beider mineralischer Rohstoffe völlig unterschiedlich sind. Während die Entwicklungsländer $z u$ über 40 Prozent an der Bauxitproduktion der westlich orientierten Staaten beteiligt sind, kommt wegen des hohen Energiebedarfs nur ein Fünftel des Hüttenaluminiums aus diesen Ländern. Kupfer dagegen wird in hohem Maße in den Entwicklungsländern verhüttet und dort auch zu einem erheblichen Teil raffiniert. ${ }^{87}$ Das Endprodukt im Kupferbergbau sind Konzentrate, bei der Metallgewinnung (Verhüttung) werden Kupferkathoden oder Drahtbarren als die Endprodukte bezeichnet. Diese Erzeugnisse zählen laut Definition noch zu den mineralischen Rohstoffen; sie werden auch von der Sysmin-Regelung erfaßt.

Angesichts dieser Schildungen wird deutlich, daß die Forderung nach einer Induzierung von Verknüpfungseffekten durch das Sysmin-Verfahren im Rahmen der beabsichtigten Zielvorgaben dieser Fazilität sicherlich überzogen ist.

Anders verhält es sich bei den sogenannten Sekundäreffekten. Definitionsgemäß sind diese Effekte für die erfolgreiche Realisierung eines Projektes selbst nicht erforderlich. Sie werden daher in der Regel bei der Pro-

85 Vgl. Pommerening, G. et al.: Möglichkeiten zur Weiterverarbeitung ausgewählter NE-Metallerze in Entwicklungsländern, ite, Hamburg, Februar 1977, S. 7; Gocht, W.: Wirtschaftsgeologie und Rohstoffpolitik, a.a.O., S. 279.

86 Vgl. Harms, U.; Iwersen, A.: Rohstoffgewinnung in Entwicklungsländern, a.a.O., S. 207.

87 Vgl. Gocht, W. (Hrsg.): Handbuch der Metallmärkte, a.a.O., S. $322 \mathrm{ff}$., S. 330 f. und S. $193 \mathrm{ff}$; Metallgesellschaft (Hrsg.): Metallstatistik 1975/1985, a.a.O., S. 12 ff. und S. 30 ff.; Mining Annual Review 1986, S. 46 und S. 27. 
jektierung und Ausführung von Kleinvorhaben nicht eingeplant und sind auch nur zum Teil beeinflußbar. ${ }^{88}$

Das Sysmin-Verfahren räumt in der Fassung von Lomé IV explizit die Möglichkeit zur Förderung derartiger Sekundäreffekte ein, z.B.: Besondere Aufmerksamkeit gilt ... der Stärkung des wissenschaftlichen und technischen Potentiales der AKP-Staaten ... (Lomé IV: Art. 216 Abs. 3).

Wie Gespräche bei der EG-Kommission und in Sambia ergeben haben, wurde die Förderung der Sekundäreffekte durch die Sysmin-Regelung bereits in den Sysmin-Anwendungsfällen in Lomé II und Lomé III angestrebt, obwohl die damals gültigen Texte des Sysmin-Verfahrens dies noch nicht explizit vorgesehen hatten.

So wurde beispielsweise für die ersten Sysmin-Darlehen an Sambia festgelegt, daß die Zinsdifferenz zwischen den Krediten an die ZCCM und den Darlehen an den Staat ${ }^{89}$ in einen Sozialfonds einzuzahlen sind, der den Bewohnern des Kupfergürtels zugute kommen muß (s. Kap. 4.3.1). ${ }^{90}$ Weiterhin bestand die Auflage, daß bei der Beschaffung von Investitionsgütern und Ersatzteilen lokale Händler eingeschaltet werden müssen. ${ }^{91}$

\subsection{Die Einflüsse der Sysmin-Regelung auf die Exportstruktur von AKP-Bergbaustaaten}

Gelegentlich wurde von europäischer Seite befürchtet, daß die AKP-Staaten versuchen könnten, unberechtigterweise auf das Sysmin-Verfahren zurïckzugreifen.

88 Vgl. Gocht, W.R.; Zantop, H.; Eggert, R.G.: International Mineral Economics, a.a.O., S. $247 \mathrm{f}$.

89 Die AKP-Staaten erhalten die Mittel und leiten sie in der Regel zu einem höheren Zins an das betroffene Unternehmen weiter.

90 Persönliches Gespräch, EG-Delegation, Lusaka 1989.

91 Persönliches Gespräch, Purchasing Manager, ZCCM Central Service Division, Kitwe 1984. 
Diese Bedenken erscheinen insbesondere dann ausgeprägt zu sein, wenn multinationale Unternehmen mit ihren - für Außenstehende nicht nachvollziehbaren - Verrechnungspreisen involviert sind. ${ }^{92}$

Weiterhin wurde seitens der Europäer die Sorge geäußert, daß durch die Sysmin-Regelung einer Vernachlässigung der Prospektion und Exploration durch die jeweiligen Bergbauunternehmen Vorschub geleistet werden könne, da ja das System im Hintergrund stünde, um im Bedarfsfall einzuspringen und Mittel zur Verfügung zu stellen. Darüber hinaus wurde unterstellt, daß Regierungen in den AKP-Ländern zur Verstaatlichung der dortigen Bergbauindustrien verleitet werden könnten, um dadurch - mittels" landesweit koordinierter Aktionen - eine Berechtigung zur Inanspruchnahme des Sysmin-Verfahrens zu erwirken. ${ }^{93}$

In diesem Zusammenhang ist auch die im Text der Sysmin-Regelung des Lomé-IV-Abkommens neu aufgenommene Formulierung: ... auf die der betreffende Staat und das betreffende Unternehmen keinen Einfluß haben ... (Lomé IV: Art. 215 Abs. 2a) zu verstehen.

Generell kann festgehalten werden, daß die Bedenken im Hinblick auf derartige Aktionen übertrieben sind. Die Attraktivität des Verfahrens wird dabei sicherlich überschätzt, denn die aus diesen Verhaltensweisen resultierenden Nachteile werden durch die Zuteilung von Mitteln der Sysmin-Fazilität nicht wieder aufgewogen. Die finanzielle Ausstattung des Fonds ist - wie der allgemeinen Kritik an den Lomé-Abkommen zu entnehmen ist - derart beschränkt, daß die Sysmin-Tranchen selbst bei großzügiger Mittelzuteilung nicht in der Lage sind, langfristig entgangene Erlöse zu kompensieren. Selbst vorübergehende Mittelengpässe können in der Regel nur mit Unterstützung von Kofinanzierungen überbrückt werden.

Auch die bei der Einführung des Sysmin-Verfahrens geäußerte Sorge, daß ein AKP-Exporteur mittels eines Lieferstopps nach der Europäischen Gemeinschaft beziehungsweise eines Umdirigierens seiner Exportprodukte von der Europäischen Gemeinschaft nach anderen Abnehmern in NichtEG-Staaten versuchen könnte, einen Exportrückgang seiner Bergbauprodukte vorzutäuschen, um so Sysmin-Darlehen zu erhalten, war bei näherer Betrachtung unberechtigt. Dennoch wurde im Text der Sysmin-Regelung

92 Persönliches Gespräch, Kommission der EG, Brüssel.

93 Persönliches Gespräch, Bundesministerium für Wirtschaft, Bonn. 
von Lomé IV die Auslöseschwelle nicht mehr an einen Exportrückgang orientiert, sondern an unter anderem der Verschlechterung der Leistungsbilanz (Lomé IV: Art. 215 Abs. 2a).

Einem AKP-Staat, der bisher nicht auf die Sysmin-Fazilität zurückgreifen konnte, bleibt allein die Möglichkeit, das Exportvolumen seiner Bergbauerzeugnisse zu vergrößern und verstärkt Abnehmer in der Europäischen Gemeinschaft $z u$ beliefern.

Bei einer Steigerung der Bergbauproduktion und damit auch des Exportes könnte die Abhängigkeitsschwelle auf den erforderlichen Prozentsatz erhöht werden. Mit der Aufnahme von Lieferungen nach der Europäischen Gemeinschaft ist die Voraussetzung für die Anspruchsberechtigung von Mitteln aus dem Sysmin-Verfahren (Lomé IV: Art. 215: ... deren Ausfuhren in die Gemeinschaft gehen) überhaupt erst gegeben (s. Kap. 4.1).

Falls eine Produktions- und Exportsteigerung eine praktikable Alternative für den AKP-Bergbau dargestellt hätte oder darstellen würde, um aus Industrieländern beziehungsweise der Europäischen Gemeinschaft günstige Mittel für den Bergbau zur Verfügung gestellt zu bekommen, ist davon auszugehen, daß während des nunmehr über zehnjährigen Bestehens der Sysmin-Fazilität der Versuch dazu unternommen worden wäre. Von solchen Versuchen ist jedoch nichts bekannt geworden. Unter den AKPStaten, die in dem Zeitraum 1979 - 1981 Sysmin-Rohstoffe nach der Europäischen Gemeinschaft lieferten, aber bei dem betreffenden Bergbauprodukt noch nicht die 15-Prozent-Abhängigkeitsschwelle ${ }^{94}$ erreicht hatten (s. Tab. 22), hat kein Land seine Produktionsmengen derart verändert verändern können -, daß die Sysmin-Regelung anzuwenden gewesen wäre.

Einige AKP-Staaten haben in dem Zeitraum 1979 bis 1981 Lieferungen von in dem Sysmin-Verfahren erfaßten Bergbauprodukten nach der Europäischen Gemeinschaft (wieder-) aufgenommen. Die Vermutung liegt nahe, daß diese Tatsache durch den Anreiz der Sysmin-Regelung eingetreten sein könnte.

Bei genauer Betrachtung jedoch stellt sich diese Hypothese als unhaltbar heraus, da bei keinem der betroffenen AKP-Staaten das jeweilige Bergbauerzeugnis die erforderliche Abhängigkeitsschwelle erreicht hat beziehungsweise kurzfristig erreichen wird. Dies resultiert nicht allein aus ei- 
Tabelle 22: Sysmin-Rohstoffe nach der EG aus AKP-Staaten, deren Exporterlöse für diese Produkte unter der erforderIichen Abhängigkeitsschwelle blieben

\begin{tabular}{|c|c|c|}
\hline AKP-Staat & $\begin{array}{l}\text { Bergbauprodukt } \\
\text { nach EG }\end{array}$ & $\begin{array}{l}\text { (wieder-) Auf nahme der } \\
\text { Lieferung nach EG }\end{array}$ \\
\hline Belize & Rohzinn & Zinn 1981 \\
\hline $\begin{array}{l}\text { Elfenbein- } \\
\text { küste }\end{array}$ & Aluminium & \\
\hline Gabun & Kupfer, raffiniert & Kupfer 1981 \\
\hline Ghana & $\begin{array}{l}\text { Bauxit; Aluminiumoxid; } \\
\text { Aluminium; Manganerz- } \\
\text { konzentrat }\end{array}$ & \\
\hline Kamerun & Aluminium & \\
\hline Kongo & $\begin{array}{l}\text { Manganerz; Kupferkon- } \\
\text { zentrat }\end{array}$ & Kupfer 1981 \\
\hline Mosambik & Mangan & \\
\hline Nigeria & Rohkobalt; Rohzinn & Kobalt 1980 \\
\hline Senegal & $\begin{array}{l}\text { Phosphat; Kupfer, } \\
\text { raffiniert }\end{array}$ & Kupfer 1981 \\
\hline Tansania & Kupfermatte; Kobalt & \\
\hline \multicolumn{3}{|c|}{ LLDC, Binnen-oder Inselstaaten: 10 \& Abhängigkeitsschwe1le } \\
\hline Benin & Zinnkonzentrat & Zinn 1981 \\
\hline Burundi & $\begin{array}{l}\text { Kupfer, raffiniert; } \\
\text { Zinnkonzentrat }\end{array}$ & Kupfex 1980 \\
\hline Fidji & Manganerz, -Konzentrat & Mangan 1981 \\
\hline Gambia & Kupfer, nicht raffiniert & Kupfer 1981 \\
\hline Guinea & Eisenerz & Eisen 1981 \\
\hline Niger & Zinnkonzentrat & \\
\hline $\begin{array}{l}\text { Papua- } \\
\text { Neuguinea }\end{array}$ & Bauxit; Aluminiumoxid & Aluminium 1979 \\
\hline Sierra Leone & Bauxit; Aluminiumoxid & \\
\hline Simbabwe & $\begin{array}{l}\text { Kupfex, raffiniert; } \\
\text { Kobalt; Rohzinn }\end{array}$ & Zinn 1980, Kupfer 1981 \\
\hline Tansania & $\begin{array}{l}\text { Kupfer, raffiniert; } \\
\text { Rohkobalt; Zinnkonzen- } \\
\text { trat }\end{array}$ & Zinn 1980 \\
\hline Uganda & Kupfer, raffiniert & \\
\hline Zaire & zinnkonzentrat & \\
\hline
\end{tabular}

Quellen: Kommission der EG (Hrsg.): Importations CEE des principaux produits, 0.0., 3. Dezember 1981; Derselbe: Analytische übersichten des AuBenhan-dels, NIMEXE, Import 1981, Bd. B und $I$, Luxemburg 1983; ebenda, NIMEXE, Import 1985, Bd. I, Luxemburg 1986 . 
nem mangelnden Interesse, wie im Falle Nigerias und Zaires, wo bereits andere Rohstoffe (Erdöl bzw. Kupfer und Kobalt) einen hohen Anteil am Exportvolumen darstellen. Bei vielen AKP-Staaten ist auch das Geopotential zu gering, oder die Projekte lassen sich aufgrund mangelnder Wirtschaftlichkeit nicht im erforderlichen Umfange entwickeln.

\subsection{Die Signifikanz der Sysmin-Regelung für den rohstoffpolitischen Bereich der Europäischen Gemeinschaft}

In marktwirtschaftlich orientierten Wirtschaftsordnungen werden prinzipiell den einzelnen Unternehmen die wesentlichen betriebsrelevanten Entscheidungen überlassen. Dies trifft auch sowohl bei der Versorgung mit mineralischen Rohstoffen zu als auch bei eventuellen Aktivitäten in vorgelagerten Bereichen, wie der Beteiligung an Rohstoffgewinnungsprojekten.

Da einer ungestörten Rohstofflieferung für die Volkswirtschaft eines Industrielandes erhebliche Bedeutung zukommt, gab es besonders in den sechziger und siebziger Jahren auch in marktwirtschaftlich orientierten Wirtschaftsordnungen Befürworter, die sich für staatliche Programme bei der Sicherstellung der Grundstoffversorgung einsetzten. ${ }^{95}$

Die Erfordernis von derartigen, begleitenden Maßnahmen ist bei den EGMitgliedstaaten beziehungsweise bei der Europäischen Gemeinschaft besonders ausgeprägt, weil:

- die Europäische Gemeinschaft einen besonders hohen Rohstoffverbrauch hat (s. Kap. 3.1);

- die Europäische Gemeinschaft einen großen Importbedarf an mineralischen Rohstoffen besitzt und sogar der weltgrößte Rohstoffimporteur ist; ${ }^{96}$

95 Vgl. Harms, U. et al.: Die Investitionspolitik ..., a.a.O., S. 360.

96 Vgl. Fuchs, G.: Bericht ..., a.a.O., S. 6; Kommission der EG (Hrsg.): Communication ..., a.a.O., S. 2. 
- die europäische Bergbauindustrie im außereuropäischen Ausland häufig ungünstigere Konditionen akzeptieren muß als die nationale Bergbauindustrie der jeweiligen Länder;

- die Risiken, sich an Bergbauprojekten der Entwicklungsländer zu engagieren, aufgrund wirtschaftlicher und politischer Unsicherheiten sowie fehlender Infrastrukturen sehr vielschichtig und schwer abschätzbar sind.

Aus diesen Überlegungen heraus wurden im Rahmen der außenwirtschaftlichen Maßnahmen Leitlinien zur Rohstoffpolitik aufgestellt sowohl von der EG-Kommission, aber auch von den einzelnen EG-Mitgliedstaaten. Dazu gehören unter anderem:

- Schaffung eines kooperativen Klimas in den Erzeugerländern;

- Reduzierung der politischen Rísiken sowie Minimierung ihrer wirtschaftlichen Auswirkungen;

- außenpolitische Unterstïtzung bei privatwirtschaftlichen Aktivitäten, Meistbegünstigungen und im Falle von auftretenden Schwierigkeiten (wie u.a. Enteignungen, Zahlungsaussetzungen);

- Diversifizierung der Versorgungsstrukturen durch Kontaktaufnahme mit potentiell neuen Bergbauländern. ${ }^{97}$

Gemäß diesen Grundsätzen bemühte sich die Europäische Gemeinschaft besonders zu Beginn der achtziger Jahre, internationale Rohstoffpolitik mit dem Schwerpunkt der Rohstoffsicherung zu betreiben, wobei den Bergbauunternehmen in den Entwicklungsländern besondere Aufmerksamkeit gewidmet wurde. ${ }^{98} \mathrm{Daß}$ in diesem Zusammenhang auch mit Staaten kooperiert wird, die nicht der AKP-Gruppe angehören, ist naheliegend, wenn man das Geopotential südamerikanischer und asiatischer Länder

97 Vgl. Gocht, W.R.; Zantop, H.; Eggert, R.G.: International Mineral Economics, a.a.O., S. 223 f.; Harms, U. et al.: Die Investitionspolitik ,.., a.a.O., S. $360 \mathrm{ff}$.

98 Vgl. Kommission der EG (Hrsg.): Memorandum zur Entwicklungspolitik der EG, Bulletin der EG 5/82, Brüssel, Mai 1982, S. 20. 
berücksichtigt. Als Beispiel für solche Aktivitäten soll hier das Engagement an dem Eisenerzprojekt Carajas, Brasilien, genannt werden. ${ }^{99}$

Bei einer Analyse der Lomé-Abkommen kann aufgezeigt werden, daß durch das Schaffen verschiedener Instrumente versucht wurde, die oben aufgefühtten Leitlinien zu realisieren (s. Kap. 2.5). Auch das Sysmin-Verfahren orientierte sich zunächst (Version Lomé II) an einem Teil der oben aufgeführten rohstoffpolitischen Grundsätze. Die seit jeher nur marginalen Ansätze in der Sysmin-Regelung zur Rohstoffsicherung der Europäischen Gemeinschaft sind zunehmend weiter in den Hintergrund getreten und in der jüngsten Version des Sysmin-Verfahrens (Lomé IV) nur noch rudimentär vertreten. So ist beispielsweise in der Lomé-IV-Version der Präambel zur Sysmin-Regelung die Bezugnahme auf die "... Ausfuhr von Bergbauerzeugnissen nach der Gemeinschaft ..." nicht mehr aufgeführt (Lomé II: Art. 49; Lomé Ill: Art. 176); es ist lediglich " ... bei der Festlegung und Durchführung der Unterstützungsmaßnahmen den beidseitigen Interessen der Vertragsparteien Rechnung zu tragen". (Lomé IV: Art. 216 Abs. 3).

Durch die Tatsache, daß in der Sysmin-Regelung von Lomé IV erstmalig eine Anpassung der Maßnahmen an die "... Umstrukturierungserfordernisse der Wirtschaft des betreffenden AKP-Staates ..." vorgeschrieben ist (Lomé IV: Art. 214 Abs. 3), wird ein Zurückstellen rohstoffpolitischer Aspekte hinter entwicklungspolitischen Belangen deutlich. Durch diese Klausel wird klar zum Ausdruck gebracht, daß selbst für die EG-Rohstoffsicherung interessante Vorhaben nur in dem Maße unterstützt werden, wie es den Umstrukturierungserfordernissen der jeweiligen AKP-Wirtschaft entspricht beziehungsweise förderlich ist.

Obwohl in dem Sysmin-Verfahren von Anfang an Komponenten zur Rohstoffsicherung enthalten waren, ${ }^{100}$ ist die besonders bei der Einführung der

99 Die Europäische Gemeinschaft für Kohle und Stahl ist mit einem Darlehen von bis zu 625 Mio. ECU (600 Mio. US-Dollar im Jahre 1982) an dem Projekt der brasilianischen Companhia Vale do Rio Doce engagiert; vgl. Kommission der EG (Hrsg.): Auswärtige Beziehungen, in: Europa-Information 63/82, Brüssel, Oktober 1982, S. 8.

100 Dies trifft auch noch für die jüngste Sysmin-Version zu: "Die ... Finanzierungsfazilität richtet sich an die AKP-Staaten, deren Ausfuhren in die Gemeinschaft gehen ..." (Lomé IV: Art. 215 Abs. 1). 
Sysmin-Regelung formulierte Behauptung, daß die Europäische Gemeinschaft von Sysmin profitiere, ${ }^{101}$ unrichtig.

Dies läßt sich wie folgt begründen:

Zwar bezieht die Europäische Gemeinschaft einige der mineralischen Rohstoffe - auch in größeren Prozentanteilen - aus den AKP-Staaten, Rohstoffe mit einem für die Gemeinschaft besonders hohen Versorgungsrisiko allerdings sind - außer Phosphaten, Kobalt und Mangan - in dem SysminKatalog nicht erfaßt; Bergbauerzeugnisse wie Chrom, Vanadium, Niob, Molybdän, Zirkon und Titan werden nur in der seit Lome III bestehenden allgemeinen Abhängigkeitsklausel (s. Kap. 2.5.3) erfaßt. ${ }^{102}$ Wird darüber hinaus berücksichtigt, daß Anfang der achtziger Jahre Phosphate aus 13 Nicht-AKP-Staaten bezogen wurden, Mangan aus 9 und Kobalt aus mindestens 8 Nicht-AKP-Staaten nach der Europäischen Gemeinschaft importiert wurden, ${ }^{103}$ dann erscheinen andere rohstoffpolitische Maßnahmen zur Sicherung der EG-Versorgung effektiver zu sein als die Sysmin-Regelung. Von einem Profitieren der Europäischen Gemeinschaft durch diese Regelung kann somit nicht die Rede sein.

Auch der weltweite Rückgang des Preisniveaus für mineralische Rohstoffe in der ersten Hälfte der achtziger Jahre kann allenfalls nur bedingt durch die EG-Rohstoffpolitik begründet werden, nicht jedoch mit dem SysminVerfahren. Vielmehr sanken die Weltmarktpreise (s. Kap. 3.4) aufgrund eines konjunkturell und technisch bedingten Nachfragerückganges sowie eines Überangebotes. Dieser Angebotsüberschuß resultierte neben dem geringeren Bedarf auf der Abnehmerseite auch aus dem Verdrängungswettbewerb auf der Produzentenseite, nachdem die bergbautreiben-

101 Vgl. u.a. Asante, S.K.B.: Lome II ..., a.a.O., S. 8; Twitchett, C.C.: Lomé II, die Enttäuschungen ..., a.a.O., S. $85 \mathrm{f}$.

${ }^{102}$ S. Tab. 9; Vgl. Gocht, W.R., Zantop, H.; Eggert, R.G.: International Mineral Economics, a.a.O., S. 219; Presse- und Informationsblatt der Bundesregierung (Hrsg.): Versorgungslage ..., a.a.O.

103 Diese Angaben schließen auch Basisstoffe, Rückgewinnungs- und Halbprodukte mit ein; Statistisches Amt der EG (Hrsg.): EC-ACPTrade, a.a.O., S. 400 ff.; Derselbe: EG-Rohstoffbilanzen 1979 1982, a.a.O., S. 119. 
den Entwicklungsländer aufgrund der Schuldenentwicklung dringend auf einen verstärkten Handel mit den Industriestaaten angewiesen waren. ${ }^{104}$

Angesichts dieses zum Teil ruinösen Wettbewerbes ist mittel- oder langfristig durchaus eine Entwicklung denkbar, wo infolge unzureichender Explorationen und Neuinvestitionen eine Angebotsverknappung eintreten könte. ${ }^{105}$ Aber auch in einem solchen Falle ist es fraglich, ob die Sysmin-Regelung Lieferengpässe im größeren Ausmaße verhindern könnten. So wurde beispielsweise die Exploration in den durch Sysmin begünstigten Abbaubetrieben der ZCCM teilweise auch nach Abwicklung der Sysmin-Transfers noch vernachlässigt. ${ }^{106}$

Ebenfalls können bei einer etwaigen Verknappung des Rohstoffangebotes aufgrund der Sysmin-Regelung keine Änderungen in der Angebotsmenge oder den Lieferströmen zugunsten der Europäischen Gemeinschaft erwartet werden.

Weiterhin rechtfertigt auch die Vereinbarung, wonach - von wenigen Ausnahmen abgesehen - ausschließlich Investitionsgüter europäischer Herkunft ${ }^{107}$ durch die Sysmin-Mittel bezogen werden können, nicht von Abmachungen zu sprechen, die ausschließlich der Europäischen Gemeinschaft zugute kämen. Weiterhin ist festzuhalten, daß in dem Zeitraum zwischen den Jahren 1975 und 1985 im Rahmen von Lomé I und Lomé II über 800 Ausschreibungen realisiert wurden im Werte von insgesamt ca. 3,7 Mrd. ECU. Davon wurden ca. 28 Prozent der Aufträge - insbesondere für Bauvorhaben - an Unternehmen aus AKP-Staaten vergeben. ${ }^{108}$ Angeboten aus den AKP-Staaten werden in den Lomé-Abkommen prinzipiell

104 Vgl. Lechner, H.; Sames, C.W.; Wellmer, F.W.: Mineralische Rohstoffe im Wandel, Essen 1987, S. 5 f.

$105 \mathrm{Vgl}$. Ebenda, S. 60.

106 Persönliches Gespräch in den Divisionen Nkana, Chingola, Mufuliera der ZCCM, Sambia; Vgl. Zambia Consolidated Copper Mines (Hrsg.): Annual Report 1983, a.a.O., S. 37.

107 Nach Lomé IV: Art. 294 werden bei den Ausschreibungen selbstverständlich auch Angebote aus den AKP-Staaten berücksichtigt; deren Produkte können aber, besonders bei hochtechnisierten Bergbauausrüstungen, nur selten mit dem europäischen Niveau konkurrieren.

108 Kommission der EG (Hrsg.): Ten years of Lomé, a.a.O., S. $57 \mathrm{f}$. 
eine Präferenz in Höhe von 10 Prozent der Angebotsvolumen eingeräumt. 109

Es kann somit festgehalten werden, daß die Vorteile, welche die Europäische Gemeinschaft aus den Ausschreibungsmodalitäten der Lomé-Abkommen zieht, im Vergleich zum EG-Welthandelsvolumen recht marginal ausfallen ${ }^{110}$ und im wesentlichen nur daraus resultieren, daß aus den AKP. Staaten (noch) keine adäquaten Angebote abgegeben werden können.

Für den rohstoffpolitischen Bereich der begünstigten AKP-Staaten besitzt die Sysmin-Regelung eine herausragende Bedeutung. Die einzelnen AKPStaat können, wenn sie die Voraussetzungen erfültt haben, auf zusätzliche Mittel mit günstigen Konditionen zurückgreifen, wodurch unter Umständen ein Rückgang der Produktion des begünstigten Unternehmens vermieden oder gemildert werden kann. Damit können eventuell langfristig bestehende Handelsbeziehungen aufrechterhalten werden, gleichzeitig ist es möglich, die Versorgung der nationalen weiterverarbeitenden Industrie zu sichern.

${ }^{109}$ Für Lieferverträge wird unter bestimmten Voraussetzungen ein K0stenvorteil von 15 Prozent eingeräumt (Lomé IV: Art. 303).

110 EG-Exporte über 420 Mrd. ECU im Jahr 1991 (über 380 Mrd. ECU in 1984); s. Abb. 3; vgl: Statistisches Amt der EG (Hrsg.): ACP basic statistics 1986, a.a.O., S. 56. 


\section{DIE SYSMIN-REGELUNG ALS ENTWICKLUNGSPOLITI- SCHES INSTRUMENT}

\subsection{Die Ansätze der Sysmin-Regelung zur Umsetzung von Strategien zur Überwindung von Unterentwicklung}

Als eine wichtige Regelung im Rahmen der Lomé-Abkommen muß sich das Sysmin-Verfahren bei einer generellen Beurteilung stets auch an den allgemeinen Zielen der Entwicklungspolitik messen lassen. Dies gilt umso mehr nachdem besonders Vertreter der EG-Kommission in der SysminRegelung nahezu ausschließlich entwicklungspolitische Belange berücksichtigt sehen. ${ }^{1}$

Wie im Kapitel 2.1 ausgeführt, gibt es unterschiedliche Ansätze zur Überwindung von Unterentwicklung. Der Versuch, die Sysmin-Regelung allein einer der Entwicklungsstrategien zuzuordnen, wäre wenig aussichtsreich. Vielmehr werden durch das Verfahren theoretisch mehrere Ansätze zur Überwindung der Unterentwicklung unterstützt sowohl direkt als auch indirekt. Dies gilt insbesondere für:

- die Strategie des unausgewogenen Wachstums,

- die Regionalentwicklungsstrategie sowie

- die Beschäftigungsstrategie.

Bereits bei der Etablierung der Sysmin-Fazilität im Jahre 1980 unterstrich die Beratende Versammlung "die Bedeutung des Systems für Bergbauerzeugnisse, dessen Heranziehung dazu beitragen kann, die Bergbauindustrien der AKP-Staaten zu erhalten und weiter auszubauen". ${ }^{2}$ Die Bereitschaft der Europäischen Gemeinschaft, den AKP-Bergbau bei der Umsetzung von Entwicklungsstrategien mitzuberücksichtigen, wurde zu Beginn der achtziger Jahre formuliert: Die Europäische Gemeinschaft will "Anstrengungen ... machen, daß die Entwicklung der Bodenschätze in den AKP-Staaten zu einem Multiplikator wird, der die Gesamtwirtschaft, die

${ }^{1}$ Persönliche Gespräche, Kommission der EG, Brüssel

2 Vgl. Focke, K.: Von Lomé 1 zu Lomé 2, a.a.O., S. 78. 
Raumordnung und die soziale Entwicklung der Länder begünstigt, die diese Bodenschätze haben". 3

Solche Absichtserklärungen wurden zum Teil auch in die Sysmin-Regelung aufgenommen, wodurch das Verfahren eine zunehmend stärkere Gewichtung im entwicklungspolitischen Bereich erhielt. So ist seit Lomé IV u.a. die Unterstützung der AKP-Bergbauländer bei der "... Diversifizierung und Erweiterung der Grundlagen für ihr Wirtschaftswachstum ..." (Lomé IV: Art. 214 Abs. 2) in dem Sysmin-Verfahren als Ziel aufgeführt.

Im Vertragstext von Lomé IV wird die Absicht der Vertragspartner, dem Sysmin-Verfahren eine wesentliche Rolle im entwicklungspolitischen Bereich zuzumessen, klar und unzweideutig dokumentiert: "Die Zielsetzung des Systems besteht darin, einen Beitrag zur Schaffung einer stabileren oder sogar erweiterten Grundlage für die Entwicklung der AKP-Staaten zu leisten ..." (Lomé IV: Art. 214 Abs. 2). Weiterhin soll das Sysmin-Verfahren zur Unterstützung von Maßnahmen dienen, "... mit welchen den schwerwiegenden volkswirtschaftlichen Auswirkungen des Verlustes an Lebensfähigkeit ( der Bergbauindustrie; Anm. d. Verfassers ) entgegengewirkt werden soll ..." (Lomé IV: Art. 214 Abs. 2,2). Darüber hinaus wurde festgelegt, daß der Beschluß über die Zuweisung der Finanzmittel "... den Bedürfnissen der wirtschaftlichen Umstrukturierung dieses AKPStaates anzupassen ist" und den "... wirtschaftlichen Interessen und den sozialen Auswirkungen einer solchen Finanzhilfe gebührend Rechnung zu tragen ..." ist (Lomé IV: Art. 216 Abs. 2).

Derartige, weit über die ursprüngliche Zielsetzung des Sysmin-Verfahrens hinausgehende Vorgaben existieren allerdings erst seit Lomé IV.

Das als "erstes entwicklungspolitische Teilziel innerhalb des Wachstumszieles" definierte "Ziel der optimalen Produktionsstruktur", ${ }^{4}$ wird ohne Zweifel auch durch die Sysmin-Regelung angestrebt. Da sich das SysminVerfahren primär auf die Bergbauindustrie konzentriert, muß somit von der indirekten Förderung eines unausgewogenen Wachstums durch die Sysmin-Fazilität ausgegangen werden.

${ }^{3}$ Vgl. AKP-EWG-Ministerrat (Hrsg.): Jahresbericht des AKP-EWG-Ministerrates, a.a.O., S. 36.

$4 \mathrm{Vgl}$. Hemmer, H.R.: Wirtschaftsprobleme der Entwicklungsländer, a.a.O., S. 61 
Eine der bei der Etablierung der Sysmin-Regelung von den AKP-Staaten vorgetragene Hauptfordenung war die Einrichtung eines Systems zur Exporterlösstabilisierung.

Die bei den meisten AKP-Staaten anzutreffende - zum Teil traditionell begründete - Konzentration auf nur wenige Exportprodukte bedingt aufgrund der teilweise erheblichen Erlösschwankungen häufig negative Konsequenzen für die Volkswirtschaft der betroffenen AKP-Staaten.

Neben intern bedingten Schwankungen der Exporterlöse können sowohl durch Nachfrager als auch durch weitere Produzenten extern bedingte Erlösschwankungen hervorgerufen werden. Die durch die Anbieterseite verursachten Preisinstabilitäten auf den Märkten für mineralische Rohstoffe sind aus verzögerten Kapazitätsanpassungen zu erklären, ebenso wie aus Störungen der Märkte, zum Beispiel durch starke Produktionserweiterungen in Entwicklungsländern, oder auch durch Lagerbestandsänderungen. Bei der Preisgestaltung agrarischer Produkte spielen ebenfalls Änderungen der Angebotsbedingungen eine große Rolle, das heißt die Preisschwankungen beruhen verstärkt auf den Wechselfällen der Produktion. ${ }^{5}$

Daher ist es naheliegend, daß vom entwicklungspolitischen Standpunkt her gesehen zur Gesundung einer Volkswirtschaft auch die Entwicklung von Fähigkeiten, auf außenwirtschaftliche Einflüsse reagieren zu können, gefördert werden muß.

Bedingt durch eine normale Preisreaktion, wobei jede Angebots- oder Nachfrageänderung auch eine Preisvariation mit sich bringt, lassen sich generell folgende kurzfristige Auswirkungen auf das Preisgefüge mineralischer Rohstoffe feststellen:

- durch politische Unwägbarkeiten - Streiks, (Bürger-)Kriege - oder Veränderungen der Vorratslagermengen seitens der Produzenten;

- durch Wechselkursänderungen, Spekulationen oder Veränderungen der Vorratslagermengen auf der Verbraucherseite.

${ }^{5} \mathrm{Vgl}$. Kommission der EG (Hrsg.): Zusammenfassender Bericht ..., a.a.O., S. 5; Schirmer, W.; Meyer-Wöbse, G.: Internationale Rohstoffabkommen, München 1980, S. 4. 
Mittel- oder langfristig sind folgende preisbeeinflussende Faktoren beobachtbar:

- Erweiterung des Angebots durch technische Innovationen, durch Erschließung neuer oder Erschöpfung alter Lagerstätten;

- Veränderung der Nachfrage, besonders durch konjunkturelle Auswirkungen, aber auch durch neue Technologien, Substitution, Materialeinsparung und in jüngster Zeit im verstärkten Maße durch Recycling sowie Umweltschutzmaßnahmen. ${ }^{6}$

Neben den oft erheblichen Preis- und Mengenschwankungen erwächst dem Problemkreis ein zusätzlicher Nachteil aus der im Vergleich zur Preisentwicklung bei Industriegütern geringeren Preissteigerungsrate der Rohstoffe. ${ }^{7}$ Diese Verschlechterung der "terms of trade", ${ }^{8}$ die sich unter anderem aus der Wertschöpfung bei der Weiterverarbeitung erklärt, ist der Entwicklung von Ländern, die mit großem Kapital- und Technologieaufwand mineralische Rohstoffe fördern und diese dann exportieren, nicht gerade dienlich.

Zur Dämpfung der Ausfuhrerlösschwankungen lassen sich mehrere theoretische Lösungsansätze unterscheiden: ${ }^{9}$

(a) Vereinbarungen zur Marktintervention

Solche - weltweit - zutreffenden Maßnahmen stellen, da durch ihre Mechanismen Einfluß auf die Preise der Rohstoffe genommen werden soll, ein System der Marktlenkung dar. Durch eine Kontrolle des An-

6 Vgl. Robbins, P.; Edwards, J.: Guide to Non-Ferrous Metals and their markets, 2. Aufl. London 1980, S. 1; Gocht, W.R.; Zantop, H.; Eggert, R.G.: International Mineral Economics, a.a.O., S. 166 ff.; Schirmer, W.; Meyer-Wöbse, G.: Internationale Rohstoffabkommen, a.a.O., S. $2 \mathrm{ff}$.

7 Vgl. Gerdes, U.; Bosse, P.: Stabex kontra Rohstoff-Fonds, a.a.0., S. 2.

${ }^{8}$ Die "terms of trade" sind definiert als Quotient aus dem Index der Exportgüterpreise und dem Index der Importgüterpreise; Vgl. Hemmer, H.R.: Wirtschaftsprobleme der Entwicklungsländer, a.a.O., S. 223.

$9 \mathrm{Vgl}$. Kommission der EG (Hrsg.): Zusammenfassender Bericht ..., a.a.O., S. 4 ff. 
gebots mittels Kontingentierung der Produktions- oder Absatzmengen beziehungsweise der Errichtung von Ausgleichslagern (bufferstocks) wird das Marktgeschehen beeinflußt.

(b) Präferenzsysteme

Hierbei handelt es sich um geographisch begrenzte Übereinkommen, worin gewisse Preisgarantien vereinbart werden.

Diese Maßnahmen werden mittels Meistbegünstigung oder Zollpräferenzen realisiert. Eine negative Begleiterscheinung dieser Absprachen ist der Aufbau weiterer Handelshemmnisse. ${ }^{10}$

Vom entwicklungspolitischen Standpunkt aus ist bei den beiden oben genannten Verfahren zu berücksichtigen, daß hierbei bestenfalls nur eine Preisstabilisierung erreicht werden kann. Ein Schwanken der Exporterlöse aufgrund von Änderungen der Nachfragemengen wird dadurch nicht verhindert, so daß exportorientierten Ländern, die unter einem Nachfragerückgang oder einem weltweiten Überangebot leiden, somit nicht geholfen werden kann. ${ }^{11}$

(c) Marktungebundene institutionelle Regelungen mit Abnahme- und Preisgarantien

Solche Verfahren verlangen die Errichtung von Organisationen, deren Funktion es ist, die vereinbarten Mengen der in den Übereinkommen erfaßten Produkte von den Produzenten aufzukaufen. Die Waren werden dann auf die Märkte gebracht, eingelagert oder "denaturiert". ${ }^{12}$

Diese nominale Exporterlösgarantie läßt sich mit einer Kaufkraftsicherung koppeln, indem die Kaufkraft für eine gewisse Menge an Industriegütern durch eine bestimmte Höhe der Exporterlöse garantiert wird. ${ }^{13}$

${ }^{10} \mathrm{Vgl}$. Kommission der EG (Hrsg.): Zusammenfassender Bericht ..., a.a.O., S. $4 \mathrm{ff}$.

11 Vgl. Kormmission der EG (Hrsg.): Zusammenfassender Bericht ..., a.a.O., S. 5.

12 Ebenda, S. 5.

$13 \mathrm{Vgl}$. Gerdes, U.; Bosse, P.: Stabex kontra Rohstoff-Fonds, a.a.O., S. 2 . 
Ein derartiger Lösungsansatz unterscheidet sich von der durch Vertreter der Entwicklungsländer häufig geforderten Indexierung der Rohstoffpreise ${ }^{14}$ dadurch, daß hier auch auf die mengenmäßig bedingten Schwankungen der Ausfuhrerlöse Einfluß genommen wird. Bei der Indexierung dagegen sollen langfristige Preisminderungen der Rohstoffe im Vergleich zu den Industriegüterpreisen verhindert werden, indem für die Rohstoffe eine Anhebung des Preisniveaus entsprechend den Steigerungen der Preise für Industriegüter vorgenommen wird. ${ }^{15}$

(d) Vereinbarungen über Ausgleichszahlungen unter Vermeidung von Markteingriffen

Diese Abkommen sollen die etwaigen Einbußen bei den Exporterlösen reduzieren. Dazu werden den betroffenen Ländern finanzielle Transfers garantiert, welche die Differenz zwischen einem festgelegten Bezugsniveau und dem tatsächlichen Wert der Exporte ganz oder teilweise ausgleichen. ${ }^{16}$

Zwei Systeme dieser sogenannten kompensatorischen Finanzierung sind seit einiger Zeit in Anwendung: Das Stabex-Verfahren der Europäischen Gemeinschaft (s. Kap. 2.5.2) und die Fazilität zur kompensatorischen Finanzierung des Internationalen Wähnungsfonds (IMF).

Die IMF-Fazilität unterscheidet sich von der Stabex-Regelung neben ihrem regional unbeschränkten Geltungsbereich im wesentlichen durch die Erfassung der Gesamtexporte - einschließlich der Dienstleistungen - eines Landes. Es werden jedoch nur die Nettoerlösausfälle eines betroffenen Staates berücksichtigt, das heißt Aufrechnung der Rückgănge mit Steigerungen in anderen Sektoren, und nicht die Bruttoerlösminderungen von einzelnen Erzeugnissen, wie es bei dem Stabex-

14 Die Indexierung ist eines der Ziele des "integrierten Rohstoffprogrammes" (UNCTAD, Commitee on Commodities, Genf 1975); Vgl. Gocht, W.: Zantop, H.; Eggert, R.G.: Internationale Mineral Economics, a.a.O., S. $197 \mathrm{f}$.

15 Vgl. Hemmer, H.R.: Wirtschaftsprobleme der Entwicklungsländer, a.a.O., S. $758 \mathrm{f}$.

16 Vgl. Kommission der EG (Hrsg.): Zusammenfassender Bericht ..., a.a.O., S. 6. 
Verfahren der Fall ist. ${ }^{17}$ Damit wird der Hauptzweck dieser IMF-Fazilität, einen Beitrag zur Behebung kurzfristiger Zahlungsbilanz-Schwierigkeiten zu leisten, deutlich. Die Höhe der Tranchen ist limitiert auf 83 Prozent der IMF-Quote des betroffenen Landes. ${ }^{18}$

\section{(e)Zusätzliche Maßnahmen}

Einen weiteren Beitrag zur Reduktion der Exporterlösschwankungen könnte die Erweiterung des Exportangebotes mit sich bringen. In dem $\mathrm{Maße}$, wie in den Entwicklungsländern eine horizontale oder vertikale Diversifikation der häufig einseitig orientierten Produktionsstrukturen vorgenommen wird, vermindert sich die Abhängigkeit von der Entwicklung eines oder weniger internationaler Märkte. Die Erlösschwankungen eines Erzeugnisses lassen sich auf diese Weise unter Umständen durch die Erlöse anderer Produkte kompensieren.

Darüber hinaus wird häufig eine Verminderung der oft extremen Exportorientierung durch eine Stärkung des binnenmarktorientierten Absatzes angeführt, wenn Konzepte zur Reduktion der negativen Auswirkungen der Exporterlösschwankungen diskutiert werden (s. Kap. 2.1).

Insbesondere der Internationale Währungsfonds knüpft die Vergabe seiner Kredittranchen an das Aufstellen von tiefgreifenden Wirtschaftsprogrammen, mit deren Hilfe unter anderem die Flexibilität einer Volkswirtschaft gegenüber externen Einflüssen gefördert werden soll. ${ }^{19} \mathrm{Zu}$ diesen Programmen zählen auch die Reduktion von staatlichen Subventionen oder eine Abwertung der Währung des jeweiligen Entwicklungslandes, wodurch sowohl eine Verringerung der Einfuhren als auch eine Erhöhung der Exporte erwartet wird. Wie die wirtschaftliche Situation der Entwicklungs- und Schwellenländer in den letzten Jahren deutlich machte, konnten derartige Wirtschaftsprogramme nur unter zum Teil erheblichen Opfern der Bevölkerung realisiert werden. Häufig war ein Anstieg der

$17 \mathrm{Vgl}$. UNCTAD (Hrsg.): Compensatory financing of export earnings shortfalls, New York 1986, S. 10 f. und 47; Bortmann, A. et al.: Ausgewählte Modelle und Reformmöglichkeiten der Exporterlösstabilisierung, Forschungsberichte des BMZ, Bd. 56, Köln 1984, S. 24 f.

$18 \mathrm{Vgl}$. UNCTAD (Hrsg.): Compensatory financing, a.a.O., S. 47.

19 Vgl. Internationaler Währungsfonds (Hrsg.): Jahresbericht des Exekutivdirektoriums für das am 30. April 1985 abgelaufene Geschäftsjahr, Washington, D.C. (1985), S. 26 - 30. 
Arbeitslosigkeit sowie eine Verknappung des Warenangebotes nicht zu vermeiden. ${ }^{20}$

In den Ländern, wo es neben anderen Maßnahmen gelang, die mit dem Internationalen Währungsfonds vereinbarten Wirtschaftsprogramme durchzuführen, konnten nach einer Anpassungsphase deutlich positive Auswirkungen auf die Entwicklung ihrer Volkswirtschaften verzeichnet werden.

Bereits bei einem Blick in den Text des Sysmin-Verfahrens läßt sich erkennen, daß die Sysmin-Regelung nicht konzipiert wurde, um zur Unterstützung einer einzelnen Entwicklungsstrategie beizutragen. Die Sysmin-Fazilität wurde nicht etabliert, um zum Beispiel die Exporterlöse zu stabilisieren oder um die Weiterentwicklung von AKP-Volkswirtschaften zu fördern. Die Sysmin-Regelung ist darüber hinaus weder in der Lage, Exporte von Bergbauerzeugnissen zu steigern noch Importe von Industriegütern zu reduzieren. Somit wird auch keine höhere Flexibilität gegenüber außenwirtschaftlichen Einflüssen bewirkt, obwohl seit Lomé III die Option besteht, Sysmin-Mittel - falls die Erhaltung der vorhandenen Kapazitäten nicht mehr möglich ist - auch zur Umstellung oder Diversifizierung in lebensfähigige Strukturen einzusetzen. Die Mittel können bei hoher Abhängigkeit der Wirtschaft von einem Bergbauprodukt auch zur Finanzierung von "Ersatzprojekten" verwendet werden (Lomé IV: Art. 216 Abs. 1). Als Beispiel sei hier die im November 1986 genehmigte Sysmin-Tranche in Höhe von 12,45 Mio. ECU an Niger erwähnt. Die Mittel werden in 2 Explorations- und Forschungsvorhaben (Gold und Kohle) eingesetzt, die der Diversifikation innerhalb der Bergbauindustrie dienen sollen. Das Kohleprojekt soll darüber hinaus dazu beitragen, den in Niger noch weit verbreiteten Einsatz von Feuerholz für Hausbrandzwecke zu reduzieren, um dadurch einer weiteren Desertifikation Einhalt zu gebieten. ${ }^{21}$

An dieser Mittelzusage wird deutlich, daß bei der Finanzierung von Diversifikationsvorhaben der ursprüngliche Katalog der Sysmin-Rohstoffe im Sinne der EG-Rohstoffsicherung keine Bedeutung mehr hat. Die wäh-

20 Vgl. z.B. Weltbank (Hrsg.): Jahresbericht 1985, Washington, D.C. (1985), S. 38 f.

21 Vgl. O.V.: Sysmin, Niger, a.a.O., S. XI; Maennig, W.: Zur Kritik des EG-Sonderfonds für Bergbauerzeugnisse (SYSMIN), in: Metall, 41. Jg., Heft 10, Oktober 1987, S. 1043. 
rend des Lomé-II-Abkommens noch ausgeprägte Rohstoffsicherungskomponente der Sysmin-Regelung nimmt mittlerweile gegenüber den entwicklungspolitischen Aspekten derartiger Finanzierungsentscheidungen eine untergeordnete Position ein. Seit Lomé IV können die Sysmin-Mittel unter gewissen Voraussetzungen sogar auch für Vorhaben außerhalb des Bergbausektors eingesetzt werden (Lomé IV: Art. 216 Abs. 1).

Die möglichen Auswirkungen, die durch die Sysmin-Regelung hervorgerufen werden können, sind nicht allein auf die AKP-Staaten beschränkt. Diese Effekte können von den Anknüpfungspunkten ausgehend mehr oder weniger stark in die einzelnen Bereiche aller beteiligten Volkswirtschaften bineinstrahlen (s. Abb. 10).

Die vorteilhaften Auswirkungen der Sysmin-Fazilität lassen sich in direkte und indirekte Effekte unterscheiden. Unter den direkten Wirkungen werden die unmittelbar mit der Auszahlung der Mittel verbundenen Nutzen verstanden, die sich eventuell in einer Verbesserung der Kapital- und Devisenbilanz des betroffenen AKP-Staates zeigen könnten. Bereits bei den bis einschließlich Lomé III ausgezahlten Sysmin-Darlehen handelte es sich trotz der Rückzahlungsverpflichtung um einen Realtransfer, da das Zinsniveau deutlich unter den Marktkonditionen lag. ${ }^{22}$

Durch Sekundär- und Multiplikatoreffekte bedingt können dann auch andere Bereiche erfaßt werden, zum Beispiel könnten positive Wirkungen auf die Beschäftigungssituation und die Infrastruktur ausgehen.

Auch in den einzelnen EG-Mitgliedstaaten stellen sich - indirekte - vorteilhafte Auswirkungen ein. Sie werden durch den Modus der Auftragsvergabe, die Sysmin-Regelung sieht eine international beschränkte Ausschreibung vor, ausgelöst. Angesichts der geringen Volumina spielen diese Effekte bei der volkswirtschaftlichen Gesamtrechnung nur eine untergeordnete Rolle.

Ebenso können internationale Rohstoffmärkte durch die Sysmin-Fazilität indirekte - Auswirkungen erfahren, denn es ist nicht auszuschließen, daß durch Aktionen der Sysmin-Regelung indirekt auch Einfluß auf die internationalen Marktkräfte ausgeübt wird. ${ }^{23}$

${ }^{22}$ Vgl. Maennig, W.: Zur Kritik des EG-Sonderfonds, a.a.O., S. 1044.

Vgl. Sames, C.W.: Anaconda, a.a.O., S. 113. 
Abbildung 10: Anknüpfungspunkte und Auswirkungen der Sysmin-Regelung in Form von Multiplikatoreffekten auf die beteiligten volkswirtschaften

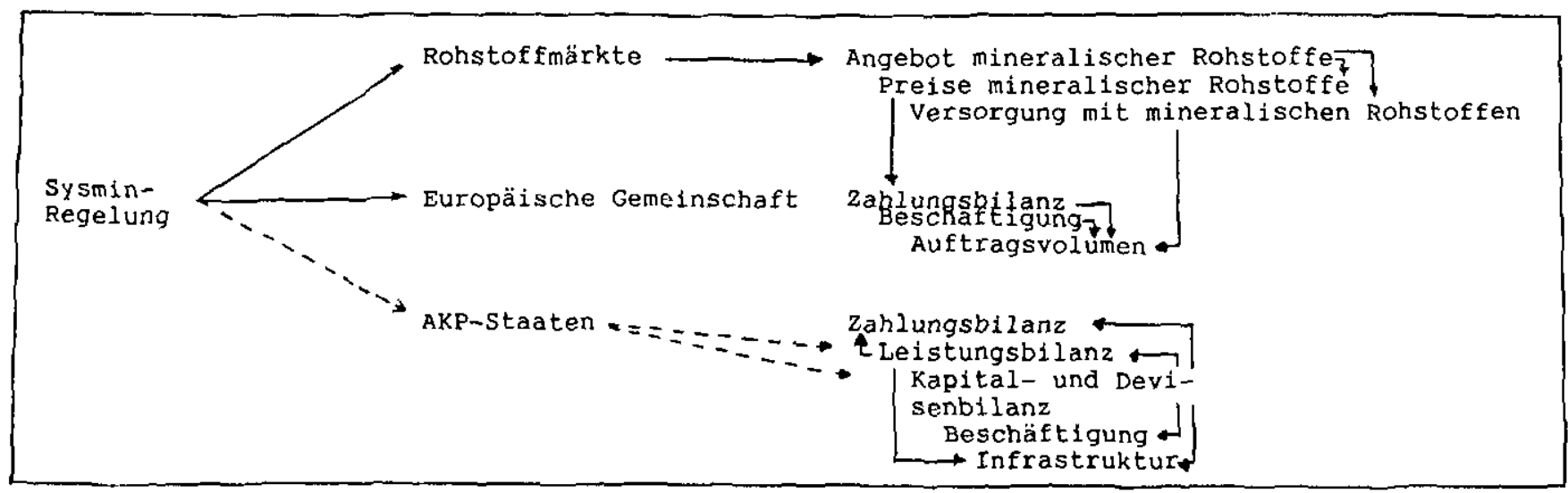

Anm.: $\quad . \quad \rightarrow$ direkte wohlfahrtsrelevante wirkung

$\longrightarrow$ indirekte wohlfahrtsrelevante wirkung

Quelle: Eigener Entwurf. 


\subsection{Die Signifikanz der Sysmin-Regelung für den entwicklungspoliti- schen Bereich}

Die Bedeutung des Sysmin-Verfahrens für die AKP-Staaten wird häufig stark unterschätzt. Dies geht besonders auch aus den vielen Publikationen über die Lomé-Abkommen hervor, wo in der Regel zwar die Existenz der Sysmin-Regelung erwähnt wird, der Stellenwert jedoch nur selten richtig eingeschätzt wird. Die völlig fehlerfreie Darstellung des immer komplizierter gewordenen Verfahrens ist nur in ganz wenigen Veröffentlichungen gelungen.

Das im Vergleich zu anderen Fazilitäten der Lome-Abkommen geringe Interesse an dem Sysmin-Verfahren erklärt sich unter anderem durch:

- das relativ kleine Volumen des Sysmin-Fonds, welches nur etwa vier Prozent des gesamten Lomé-IV-Etats ausmacht (s. Tab. 1);

- die große Anzahl von AKP-Staaten, welche die Fazilität nicht in Anspruch nehmen können sowie

- die unproblematische und damit unspektakuläre Abwicklung der Mittelzuteilung und -verwendung.

Dennoch kann die Sysmin-Fazilität als eine der wichtigsten Einrichtungen in den Lomé-Abkommen angesehen werden, für die in Frage kommenden AKP-Bergbauländer als die wichtigste schlechthin. Diese Tatsache resultiert nicht nur aus den vorgenommenen Optimierungen und Erweiterungen der Regelung, sondern auch daraus, daß den AKP-Staaten neben günstigen Finanzmitteln auch technischer Rat sowie spezielles Controlling für ihre wichtigsten Schlüsselindustrien zuteil wird. Das heißt es wird ein Industriezweig unterstützt, der im Lande bereits existiert und wo langjährige Erfahrungen und Kenntnisse vorliegen, die ausbaufähig sind.

Trotzdem bietet das Sysmin-Verfahren kein allgemeines Lösungskonzept für die Entwicklung von AKP-Bergbauländern. Das wenig attraktive Investitionsklima, welches immer noch in den meisten AKP-Staaten vorherrscht und häufig auf politische Instabilitäten zurückzuführen ist, kann durch die Sysmin-Regelung nicht verändert werden.

Den europäischen Bergbauunternehmen - dies gilt ebenso für andere Industriezweige - fehlen beispielsweise in Afrika grundlegende Voraussetzun- 
gen, insbesondere Infrastruktur, aber auch wirtschaftliche und politische Sicherheiten, um sie zu verstärkten Investitionen zu veranlassen..$^{24}$ Bei den empirischen Erhebungen in Sambia wurde dieser Sachverhalt deutlich, selbst in dem für die Verhältnisse in Schwarzafrika weit entwickelten Kupfergürtel.

Wie die Erhebungen in den Kupferbergwerken Sambias weiterhin ergeben haben, sind die unter Lome II und Lomé III realisierten Sysmin-Transfers im Hinblick auf entwicklungspolitische Zielsetzungen noch nicht optimal eingesetzt worden. Darüber hinaus könnte den in der Sysmin-Regelung von Lomé IV formulierten Zielen durch konkretere Formulierungen ein noch stärkerer Stellenwert im Hinblick auf die entwicklungspolitische Bedeutung eingeräumt werden. Dabei ist jedoch $z u$ berücksichtigen, daß durch zu präzise Vorgaben individuelle Modifikationen kaum noch möglich wären. Angesichts der heterogenen Verhältnisse in den einzelnen AKP-Staaten sowie der differenzierten Belange im Bergbau selbst ist zur optimalen Wirkung der Sysmin-Mittel das Vorhandensein von gewissen Freiräumen unbedingt erforderlich.

Prinzipiell versprechen sich Politiker und Entwicklungsexperten durch das verstärkte Erschließen von Bodenschätzen beziehungsweise durch die Steigerung der Bergbauproduktion eine Vielzahl von volkswirtschaftlich vorteilhaften Effekten:25

- Steuermehreinnahmen oder die Möglichkeit, bei konstanten Steuereinnahmen anderen Wirtschaftsbereichen Steuerentlastungen einzuräumen;

- Zunahme der Investitionen;

- Steigerung der Devisenerlöse;

- Ausbau der Infrastuktur, speziell in weniger entwickelten Regionen;

24 Vgl. AKP-EWG-Ministerrat (Hrsg.): Jahresbericht des AKP-EWGMinisterrates (1982), Brüssel, S. 36.

25 Vgl. Gocht, W.R.; Zantop, H.; Eggert, R.G.: International Mineral Economics, a.a.O., S. 248 ff.; Europäische Investitionsbank (Hrsg.): 25 Jahre 1958 - 1983, o.O., April 1983, S. 88; Pommerening, G. et al.: Möglichkeiten ..., a.a.O., S. 179; Kommission der EG (Hrsg.): Communication ..., a.a.O., S. 2. 
- Verbesserung der Beschäftigungssituation;

- Vermehrte Aus- und Weiterbildung von Arbeitskräften;

- Erschließung der Möglichkeit von Weiterverarbeitung;

- Besseren Anschluß an technische Entwicklungen.

Da die Realisierung dieser Effekte theoretisch durchaus möglich ist, werden diese Punkte oft als Argument für das Durchführen von Entwicklungsvorhaben angeführt. In praxi jedoch sieht die Situation häufig ganz anders aus. Die einzelnen Auswirkungen fallen in der Regel wesentlich geringer aus als es eine oberflächliche Betrachtung erwarten läßt, denn die Effekte werden immer von anderen - unvorteilhaften - Wirkungen überlagert. In vielen Fällen ergeben sich somit häufig nur schwach positive Auswirkungen, gelegentlich überwiegen sogar die Negativeffekte. In dieser Arbeit wird auf die von vornherein ungünstigen Einflüsse des Bergbaues auf eine Volkswirtschaft (z.B. durch Umweltbelastung, die Methoden der Landnahme, soziale Strukturveränderungen) nicht näher eingegangen.

Ein Anstieg der Devisenerlöse macht sich erst in einem recht späten Zeitpunkt bemerkbar, da Deviseneinnahmen in der ersten Periode der Betriebsphase zunächst durch Kapitalabflüsse für den Import von Produktionsfaktoren und Dienstleistungen kompensiert werden.

Dies erklärt sich aus der Kapitalintensität des modernen Bergbaues und der Notwendigkeit, immer anspruchsvollere Abbautechnologien einsetzen zu müssen. Die dafür erforderlichen Investitionsgüter werden in der Regel nicht in den Entwicklungsländern produziert und die Anschaffungen wirken sich, sofern sie nicht durch ausländische Partner importiert werden, daher negativ auf die Zahlungsbilanz des Entwicklungslandes aus. In der Bauphase ist der passivierende Effekt somit größer als in der anschließenden Produktionsphase. ${ }^{26}$ Aber auch während der Betriebszeit kann ein erheblicher Mittelabfluß für den Schuldendienst und die Beschaffung von Betriebsstoffen und Ersatzteilen entstehen. Dies läßt sich besonders deut-

${ }^{26} \mathrm{Vgl}$. Harms, U.; Iwersen, A.: Rohstoffgewinnung in Entwicklungsländern, a.a.O., S. 218; Schützdeller, R.: Sekundäreffekte von Bergbauprojekten, Diss. RWTH Aachen, Internationale Kooperation, Bd. 25, Baden-Baden 1984, S. 6. 
lich am Beispiel Papua-Neuguineas darlegen, wo das Bergbauprojekt Ok Tedi (Phase 1: Aufnahme der Goldproduktion 1984; Phase 2: Aufnahme der Kupferproduktion 1986) einen beachtlichen Einfluß auf die Wirtschaft dieses AKP-Staates ausübt. Nach Ende der 1. Entwicklungsphase im Jahre 1984 reduzierten sich die Importe für den Ok Tedi-Bergbau zunächst von 7,9 Prozent des Bruttosozialproduktes auf 1,9 Prozent im Jahre 1985, um dann bis zum Jahre 1987 wieder auf 7,0 Prozent des Bruttosozialproduktes (Schätzung) anzusteigen. Obwohl sich die Exporterlöse Papua-Neuguineas seit dem Jahre 1982 nahezu verdoppelt haben, ist seit dem Jahre 1985 wieder eine zunehmend negative Leistungsbilanz zu verzeichnen, da sowohl die Importe stark zugenommen haben (1985 bis 1988: plus ca. 60 Prozent) als auch ein steigender Schuldendienst - auch für Ok Tedi geleistet werden muß.27

Bei der sambischen Kupfergesellschaft ZCCM, mußten im Jahre 1984 beispielsweise über 10 Prozent der Deviseneinnahmen für Ersatzteilkäufe im Ausland aufgewendet werden. ${ }^{28}$

Dieser Devisenabfluß macht sich besonders gravierend bemerkbar, wenn sich die Exporterlöse - aufgrund sinkender Marktpreise oder eines geringeren Marktabsatzes - nicht gemäß den Prognosen entwickeln.

In solchen Fällen wären Steuerentlastungen für die betroffenen Unternehmen, zum Beispiel durch die Anerkennung von steuermindernden Aktionen und die Möglichkeit der Aufrechnung gegen Steuerverbindlichkeiten in anderen Geschäftsbereichen, von großem Nutzen.

Doch die verschuldeten Entwicklungsländer können oder wollen in der Regel nicht auf Steuereinnahmen verzichten. Das Kupferbergwerk Panguna auf der Insel Bougainville beispielsweise trug vor seiner Schließung 17 Prozent zu den Staatseinnahmen Papua-Neuguineas bei. ${ }^{29}$ Die Regierungen in den Entwicklungsländern können sich daher nur schwer entschließen, die in finanziellen Schwierigkeiten befindlichen Unternehmen

27 Angaben für 1988 geschätzt; Commenwealth Fund for Technical Cooperation (Hrsg.): Papua New Guinea; Informationsmemorandum, o.O., März 1988, S. 13 f., 18 ff.

$28 \mathrm{Vgl}$.Pagni, L.: Getting out of the copper straitjacket, a.a.O., S. 19.

29 O.V.: Die Minen-Krise stürzt die Wirtschaft in Ungewißheit, in: Handelsblatt, 23. Januar 1990. 
durch Steuerminderungen zu entlasten. Ein solches Verhalten konnte auch in Sambia im Falle der ZCCM beobachtet werden.

Der aus Bergbauvorhaben resultierende Investitionsanstieg induziert nicht automatisch Auswirkungen auf andere Wirtschaftszweige. Prinzipiell läßt sich feststellen, daß die Verknüpfungs-Effekte - wie auch die empirischen Erhebungen in Sambia gezeigt haben - geringer sind als erwünscht (vgl. Kap. 4.4).

Die Infrastrukturmaßnahmen sind zum Teil von erheblicher Bedeutung, berīcksichtigen im wesentlichen jedoch nur die Belange des jeweiligen Bergbauprojektes. Abgesehen von der Nutzung von Straßen durch die Bevölkerung der Region und einer eventuellen Elektrifizierung von Ansiedlungen, kommen die Infrastrukturmaßnahmen im wesentlichen nur den Beschäftigten des Bergbaubetriebes zugute; der Rest der Bevölkerung profitiert in der Regel nicht von solchen Maßnahmen. Dies trifft beispielsweise für Schulen zu, die auf dem Werksgelände liegend nur für die Kinder der Mitarbeiter zugänglich sind. Das gleiche gilt für werkseigene Hospitäler, die durch ihre separierte Lage auf dem firmeneigenen Gelände nicht für jedermann offen sind, für Sportanlagen oder für Personentransporte von den Wohnsiedlungen zu den Arbeitsstätten.

Auch zur Verbesserung der Beschäftigungssituation sind kritische Bemerkungen angebracht.

Allgemein sind die Auswirkungen auf die Beschäftigung bei Bergbauprojekten in Entwicklungsländern gering. ${ }^{30}$ In der Regel verändert sich das Beschäftigungsniveau im Bergbau - abgesehen. von Neuerschließungen oder Produktionseinstellungen - nur unwesentlich.

Vor allem bei neuen Projekten wird der Mitarbeiterstamm fürs erste aus anderen Betrieben rekrutiert, um so in der kritischen Entwicklungsphase die Personalkosten möglichst gering zu halten. Dies gilt besonders für Großunternehmen. ${ }^{31}$

${ }^{30} \mathrm{Vgl}$. O.V.: Die Minen-Krise stürzt die Wirtschaft in Ungewißheit, a.a.O.; Harms, U.; Iwersen, A.: Rohstoffgewinnung in Entwicklungsländern, a.a.O., S. 260.

${ }^{31}$ Auch in den Entwicklungsländern sind Personalkosten kein vernachlässigbarer Posten; Vgl. Zambia Consolidated Copper Mines (Hrsg.): Annual Report 1983, a.a.O., S. 15. 
Auch dürfen die Beschäftigungseffekte in den Zulieferindustrien nicht überschätzt werden, da die bereits etablierten Unternehmen versuchen werden, die Auftragszunahme ohne Personalneueinstellung zu bewältigen. Dies trifft besonders in einer rezessiven Konjunkturphase zu, wo die vorhandenen Kapazitäten noch zusätzliche Engagements zulassen.

Die vermehrte Aus- und Weiterbildung von Mitarbeitern und deren Angehörigen ist ein weiterer Punkt, der zu den vorteilhaften Effekten gezählt wird.

Häufig jedoch bleibt auch in diesem Falle die Realität hinter den Erwartungen der Entwicklungspolitiker zurück, obwohl besonders in den großen Bergbauunternehmen, die auch Ausbildungs- und Lehrwerkstätten betrejben, Kurse und Lehrgänge abgehalten werden. Meistens aber handelt es sich um einsatzbezogene Schulungsprogramme wie "Arbeitsweise des hydraulischen Baggers" oder "Theorie und Praxis von Kettenfahrzeugen", ${ }^{32}$ die ein bis zwei Tage dauern, maximal eine Woche.

Auf der anderen Seite fehlt es aber fast immer an längeren und qualifizierten Lehrgängen für Mitarbeiter in mittleren und gehobenen Positionen sowie an Ausbildungsprogrammen für zukünftige Mitarbeiter. Im Jahre 1983 zahlte die sambische Kupfergesellschaft ZCCM 345 Studenten und Angestellten ein Auslandsstipendium. ${ }^{33}$ Gewiß eine beachtliche Zahl, bei nahezu 60.000 Mitarbeitern, jedoch ergibt sich nur ein Verhältnis von bescheidenen 0,5 Prozent. In Anbetracht der chronischen Unterbesetzung in der mittleren und gehobenen Angestelltenebene - Geologen, Ingenieure von 10 bis 20 Prozent seit Beginn der "Sambianisierungs-Phase" im Jahre $1964^{34}$ ist die Anzahl gering. Ob sich bei Neu- oder Erweiterungsprojekten wesentliche Effekte in diesen Richtungen entwickeln, ist aufgrund der Erhebungen in Sambia mit großer Skepsis zu hinterfragen.

Damit sich der sogenannte "managerial und technological gap" zu den Industrieländern nicht noch weiter vergrößert, ist es für die Entwicklungsländer wichtig, daß sie den Anschluß an den technischen Fortschritt finden und halten. Jedoch kommt das im Rahmen von Entwicklungsprojekten übermittelte technische Wissen - wie auch während der Erhebung in Sam-

32 Vgl. Schützdeller, R.: Sekundäreffekte ..., a.a.O., S. 219, Tabelle 21.

33 Diese Stipendien wurden zum Teil aus Mitteln der Sysmin-Fazilität bezahlt; Persönliches Gespräch, EG-Delegation, Lusaka, Sambia.

34 Persönliches Gespräch, Nkana, ZCCM, Kitwe, Sambia. 
bia deutlich wurde - hāufig nur engen Spezialbereichen zugute und erfährt somit nicht die erwünschte, weite Verbreitung in sämtliche Bereiche des Entwicklungslandes.

Ebenso kritisch ist der - entwicklungspolitisch häufig geforderte - Einsatz moderner Verfahrenstechniken zu betrachten.

Zwar können mit Hilfe moderner Verhüttungs-, Raffinations- und Weiterverarbeitungstechniken Bergbauerzeugnisse im Produktionsland weiter veredelt werden, ob sich dadurch jedoch auch positive Effekte für die Volkswirtschaft einstellen, hängt von der Marktsituation ab.

So ist es fraglich, ob die weiterverarbeiteten Produkte in den Industrieländern überhaupt in dem Maße wie die Grundstoffe nachgefragt werden, da die Importeure, um ihre eigenen Kapazitäten auszulasten, in der Regel auch weiterhin an einer Belieferung mit Grundstoffen interessiert sind.

Desweiteren ist es zweifelhaft, ob die geforderten Preise für die im Rohstoffland weiterverarbeiteten Produkte überhaupt mit dem Weltpreisniveau konkurrieren können. Standortnachteile, wie hohe Energiekosten, ungenügende Infrastruktur oder geringe Verarbeitungsmengen, bedingen häufig Kosten in einer derartigen Höhe, daß die Erzeugnisse nur noch zu Preisen angeboten werden, die nicht mehr wettbewerbsfähig sind.

Kann und soll nun die Sysmin-Regelung bei diesen oben aufgelisteten Sachverhalten eine Verbesserung bewirken?

Sicherlich sind die Erwartungen hochgeschraubt worden: Im $\mathrm{Zu}$ sammenhang mit der Bergbau-Fazilität wurde von Auswirkungen gesprochen, die sich für die Industrialisierung der AKP-Länder als positiv erweisen würden; ${ }^{35}$ die EG-Kommission erklärt in einem Bericht an den AKP-EWG-Ministerrat: "Sysmin habe sich als sehr wirksam erwiesen ... und konnte auf diese Weise den Verfall der Produktionskapazitäten aufhalten". ${ }^{36}$

Derartige Aussagen sind in dieser generalisierenden Form sicherlich übertrieben und unzweckmäßig. Denn das auf diese Weise von den EGOrganen im Hinblick auf die Sysmin-Fazilität erzeugte Anspruchsdenken

${ }^{35} \mathrm{Vgl}$. Fuchs, G.: Bericht ..., a.a.O., Teil B, S. 37.

${ }^{36}$ Vgl. Kommission der EG (Hrsg.): Bericht ..., a.a.O., S. 11. 
bewirkt, daß durch die Sysmin-Regelung ausgelöste Positiveffekte in der Erwartung von größeren Auswirkungen nur bedingt wahrgenommen werden.

Um die unbestritten positiven, aber auch negativen mikro- und makroökonomischen Wirkungen der Sysmin-Regelung möglichst deutlich und relativiert herauszuarbeiten, wird bei der nachfolgenden Betrachtungsweise zwischen den begünstigten Bergbauunternehmen und dem AKP-Staat differenziert (s. Tab. 23).

Tabelle 23: Konditionen und Auswirkungen der Sysmin-Regelung auf das Bergbauunternehmen und den AKP-Staat

\begin{tabular}{|c|c|c|}
\hline & $p \circ s i t i v$ & negativ \\
\hline Bergbauunternehmen & $\begin{array}{l}\text { Vermögens- und Kapital- } \\
\text { zunahme } \\
\text { - günstige Darlehenskon- } \\
\text { ditanen } \\
\text { - indirekte steigerung der } \\
\text { Kreditwürdigkeit } \\
\text { - Bezug von Bergbauaus- } \\
\text { rüstungen und Moderni- } \\
\text { siexung von Anlagen }\end{array}$ & $\begin{array}{l}\text { - Auflagen durch die EG- } \\
\text { Kommission } \\
\text { - lange Beazbeitungszeit } \\
\text { der Anträge } \\
\text { - längere Belieferzeit- } \\
\text { räume }\end{array}$ \\
\hline AKP-Staat & $\begin{array}{l}\text { - Einsparung von zuschüs- } \\
\text { sen und Subventionen } \\
\text { - Staatsmittel stehen ande-- } \\
\text { ren Sektoren zut verfü- } \\
\text { gung } \\
\text { - Kontinuität bei Royalties } \\
\text { und anderen Steuereinnah- } \\
\text { men }\end{array}$ & $\begin{array}{l}\text { - Steigerung des aus- } \\
\text { ländischen Einflusses }\end{array}$ \\
\hline
\end{tabular}

Quelle: Eigener Entwurf.

Als größter Vorteil der Sysmin-Fazilität, sowohl für das begünstigte Unternehmen als auch für den AKP-Staat, zählt die extrem günstige Bereitstellung von Finanzmitteln. Diese Feststellung traf auch für die Vergabepraxis vor Lomé IV zu, als die Mittel aus der Sysmin-Fazilität hoch als Sonderdarlehen vergeben wurden.

Neben Devisenabflüssen für Importe von Anlagen und Dienstleistungen, Hilfs- und Betriebsstoffen, Ersatzteilen, Abflüsse für Gehälter und Divi- 
dende wirken sich besonders die Schuldendienstzahlungen für ausländisches Fremdkapital ungünstig auf die Zahlungsbilanz eines Entwicklungslandes aus. ${ }^{37}$

Bereits die Darlehen der Sysmin-Fazilităt während der Laufzeit von Lomé II und Lomé III kamen den AKP-Staaten aufgrund der vierzigjährigen Laufzeit, der zehnjährigen tilgungsfreien Zeit und des niedrigen Zinses, der mit 1 Prozent weit unter dem Marktzins für ein Entwicklungsland lag, sehr entgegen. Trotz der Rückzahlbarkeit handelte es sich bei diesen Sysmin-Darlehen angesichts der Zinssubventionen bereits um Realtransfers von der Europäischen Gemeinschaft in die begünstigten AKP-Staaten. ${ }^{38}$ Durch die seit Lomé IV nunmehr als Zuschüsse zur Verfügung gestellten Sysmin-Mittel wird sich der positive Effekt wesentlich erhöhen. Das Einsparen von staatlichen Mitteln, die sonst aktiviert werden müßten, um ein Bergbauunternehmen mittels Darlehen, Zuschüssen und Subventionen vor Kapitalengpässen zu bewahren, kann sich auch auf andere Wirtschaftszweige positiv auswirken.

Neben der gelegentlich geäußerten Sorge vor einer Zunahme des ausländischen Einflusses mag für manche AKP-Regierung bedauerlich sein, daß die Mittel nicht - wie bei der Stabex-Regelung - zu ihrer Disposition stehen. Die Realisierung eigener regional- oder strukturpolitischer Konzepte über Rehabilitierungsprogramme hinaus kann nur bedingt mit Hilfe des Sysmin-Verfahrens durchgeführt werden, da die Konditionen eine mit den Zielen der Regelung in Einklang stehende Verwendung der Transfers vorsehen (Lomé IV: Art. 214).

Den begünstigten Bergbauunternehmen können durch die Sysmin-Regelung Darlehen zu Vorzugskonditionen zur Verfügung gestellt werden. Zinssätze und Schuldendienstregelungen sind dabei weit günstiger als unter den Konditionen der internationalen Kapitalmärkte. Während der Laufzeit von Lomé II und Lomé III wurde die Zinsdifferenz (etwa 5 Prozent) zwischen dem Zinssatz, den das Unternehmen an den AKP-Staat entrichten mußte, und dem Satz, den der Staat an die EG-Kommission zu leisten hatte, in der Regel zum Aufbau eines Sozialfonds verwendet

${ }^{37} \mathrm{Vgl}$. Hemmer, H.R.: Wirtschaftsprobleme der Entwicklungsländer, a.a.O., S. 176; Harms, U.; Iwersen, A.: Rohstoffgewinnung in Entwicklungsländern, a.a.O., S. 224.

Vgl. Maennig, W.: Kritik des EG-Sonderfonds, a.a.O., S. 1044. 
(s. Kap. 4.3.1). ${ }^{39}$ Die Bevölkerung des AKP-Staates, wie die Erhebungen in Sambia ergaben, meistens die Bergleute des begünstigten Abbaubetriebes und deren Familien, profitiert also ebenfalls zu einem gewissen Maße von der Sysmin-Fazilität.

Darüber hinaus konnten die Sysmin-Darlehen, da die Laufzeit der Kredite an die begünstigten Abbaubetriebe unter der vierzigjährigen Laufzeit der Sysmin-Transfers an die AKP-Staaten liegen (etwa 10 - 20 Jahre), nach ihrer Rückzahlung an den AKP-Staat nochmals zur Finanzierung von (Entwicklungs-)Vorhaben in dem jeweiligen AKP-Land eingesetzt werden. ${ }^{40}$ Dies trifft natürlich in vollem Umfange auch auf die Sysmin-Zuschüsse zu.

Häufig wurde der Sysmin-Regelung - wie überhaupt dem gesamten Komplex des Lomé-Abkommens - eine zu geringe Mittelausstattung unterstellt. ${ }^{41}$

Richtig ist, daß die Antragsteller in der Regel mehr Mittel benötigen, als sie aus dem Fonds zugeteilt bekommen. So beliefen sich die ersten Anträge der AKP-Staaten Sambia und Zaire auf insgesamt 140 Mio. ECU. Sie bekamen aber lediglich 95 Mio. ECU aus dem Sysmin-Fonds zugesprochen. ${ }^{42}$

Im Jahre 1982 betrugen allein die Investitionen der sambischen Regierung im Kupferunternehmen ZCCM über 300 Mio. ECU, ${ }^{43}$ im Vergleich dazu sind die 55 Mio. ECU des Sysmin-Darlehens nur ein marginaler Betrag.

39 Persönliches Gespräch, ZCCM-Headoffice, Lusaka; EG-Delegation, Lusaka, Sambia.

40 Persönliches Gespräch, Kommission der EG, Generaldirektion Entwicklung, Brüssel.

41 Vgl. u.a. Sefali, M.: A significant contribution to human understanding and development, in: The Courier, Nr. 120, März - April 1990, S. 7; Rajana, C.: Europe undermined, a.a.O., S. 27.

42 Vgl. Insanally: Bericht im Namen des Paritätischen Ausschusses über den Fünften Jahresbericht des AKP-EWG-Ministerrates, Beratende Versammlung AKP-EWG Sitzungsdokumente 1981 - 1982, 12. Oktober 1981, S. 34.

43 VgI. O.V.: Zambia, in: Africa Research Bulletin, 15. Januar - 14. Februar 1983, S. 6743. 
Bei der Kritik ist zu beachten, daß die Sysmin-Regelung von der europäischen Seite nur als Kofinanzierung verstanden wird, d.h. es ist beabsichtigt, nur einen gewissen Teil der erforderlichen Mittel bereitzustellen. Andere Institutionen und private Kapitalgeber sollen ebenfalls einen Beitrag leisten. Außerdem existieren im Rahmen der Lomé-Abkommen neben dem Sysmin-Verfahren - wie erwähnt - zur Unterstützung der Bergbauindustrie in den AKP-Staten noch weitere Fazilitäten (s. Kap. 2.5.4 und Tab. 4).

Aus diesem Grunde ist auch die Rolle der Europäischen Investitionsbank auf dem Sektor der Bergbauvorhaben seit dem Lomé-Il-Abkommen aufgewertet worden. ${ }^{44}$

In diesem Zusammenhang sind die Darlehen dieser Bank an die sambische Kupfergesellschaft ZCCM (s. Kap. 4.3.1) zu erwähnen, welche seit dem Jahre 1981 - unabhängig von der Sysmin-Tranche - gewährt wurden. Es handelt sich unter anderem um 25 Mio. ECU, die mit weiteren Darlehen der Weltbank, des Internationalen Währungsfonds und der Afrikanischen Entwicklungsbank für ein Projekt zur Aufarbeitung der Abraumhalden des Nchanga-Bergwerkes bereitgestellt wurden. ${ }^{45}$

Neben der indirekten Erhöhung des disponiblen Kapitals ${ }^{46}$ macht sich die "Signalwirkung" der Darlehen an das begünstige Unternehmen besonders positiv bemerkbar. Weitere Kreditgeber, auch Lieferanten, haben bei einer Kofinanzierung in der Regel die Gewähr, daß sie sich in einem prinzipiell lebensfähigen Unternehmen engagieren, da gemäß dem Sysmin-Reglement die Mittel nur zur "Aufrechterhaltung, Wiederherstellung oder Rationalisierung der betreffenden Produktions- oder Ausfuhrkapazitäten auf einen wirtschaftlich lebensfähigen Stand" bestimmt sind (Lomé IV: Art. 216 Abs. 1).

Ungünstig sowohl für die Bergbaugesellschaft als auch für den AKP-Staat wirkten sich die langen Entscheiđungszeiträume über die Zulässigkeit eines Sysmin-Antrages sowie die Höhe der Mittelzuteilung aus. Zum Beispiel wurde der AKP-EWG-Ministerrat bereits auf seiner 7. Tagung, am

44 Vgl. Rajana, C.: Europe undermined, a.a.O., S. 29.

45 Persönliches Gespräch, Nchanga-Division, ZCCM, Chingola, Sambia; Kommission der EG, Brüssel.

${ }^{46}$ Die Sysmin-Darlehen sind nicht frei disponibel, jedoch werden durch ihre Gewährung andere Mittel frei verfügbar. 
13. und 14. Mai 1982, davon in Kenntnis gesetzt, daß 2 weitere AKPStaaten, Guayana und Ruanda, Interesse gezeigt hătten, einen Sysmin-Antrag zu stellen.

Ende Juli 1984 wurden zwar 3 Mio. ECU als Vorauszahlung für die Umstrukturierung beziehungsweise Modernisierung der Bauxitvorkommen auf Guayana freigegeben, ${ }^{47}$ eine Entscheidung über die Höhe des gesamten Sysmin-Transfers konnte jedoch nicht getroffen werden, da der EGKommission noch kein ausgearbeitetes Rehabilitationsprogramm vorlag (s. Kap. 4.2). Auch im Falle Ruandas dauerte es bis Ende 1984, bis dem AKP-Staat ein Sysmin-Darlehen für seinen handwerklich betriebenen Zinnbergbau zugebilligt wurde. ${ }^{48}$

Hier zeigt sich deutlich, daß besonders kleinere und mittlere Bergbauunternehmen, aber auch die weniger entwickelten AKP-Staaten, die nicht auf eine an systematisches Arbeiten gewöhnte Beamtenschaft zurückgreifen können, benachteiligt sind. An dieser Tatsache ändern auch die in nahezu allen AKP-Staaten vertretenen EG-Delegationen nichts. Die in der Modifikation des Sysmin-Verfahrens in Lomé IV vorgenommene Zusammenlegung der beiden Genehmigungsphasen (Lomé IV: Art. 217 Abs. 3) wird zwar zur Verkürzung des Antragsverfahrens beitragen, löst jedoch die hier angesprochenen Probleme einzelner AKP-Staaten, zum Beispiel bei der Erarbeitung von Rehabilitationsprogrammen, nicht.

Neben diesen Perioden der Ungewißheit über die Höhe der zugebilligten Sysmin-Mittel wirkt sich für die betroffenen Bergbaugesellschaften auch der teilweise erheblich ausgedehnte Zeitraum bis zum Eintreffen der ausgeschriebenen und dringend benötigten Bergbauausrüstungen aus.

Zum einen ist diese Tatsache durch die zeitintensiven Vorarbeiten ${ }^{49}$ bedingt, zum anderen aber durch die Ausschreibungsmodalitäten, die - obwohl großzügig gehandhabt - mehr Zeit beanspruchen als dies bei einer Direktbeschaffung der Fall wäre. Dieser Nachteil wurde während der empirischen Erhebungen in Sambia besonders deutlich.

47 Persönliches Gespräch, Kommission der EG, Brüssel. Vgl. Kommission der EG (Hrsg.): Mitteilungen an die Presse, IP (84) 347, 11. Oktober 1984.

48 Persönliches Gespräch, Bundesministerium für Wirtschaft, Bonn.

49 Lomé IV: Art. 217 Abs. 2: Vor jeder Entscheidung der Gemeinschaft wird systematisch eine ... Diagnose ... durchgefuhrt. ... 
Des weiteren wird auch die Beschäftigungssituation von der Bergbau-Fazilität beeinflußt. So kann davon ausgegangen werden, daß in den begünstigten Abbaugesellschaften ein Teil der Mitarbeiter durch die SysminMittel vor der Entlassung bewahrt wird.

Angesichts der zusätzlichen Finanzmittel ist es dem betroffenen Unternehmen eher möglich, den Mitarbeiterstamm zu halten beziehungsweise nicht in dem Maße Entlassungen vorzunehmen, wie es ohne die Sonderdarlehen erforderlich wäre.

Dies trifft auch auf Beschāftigte in den Zulieferbetrieben zu, da die Europäische Gemeinschaft die Sysmin-Mittelvergabe an die Auflage koppelt, bei der Gerätebeschaffung lokale Vertragshändler mit einzubeziehen. Diese Bedingung stößt zwar bei den Bergbauunternehmen nicht immer auf große Sympathie, ${ }^{\text {so }}$ vergrößert aber in nicht unerheblichem Maße die entwicklungspolitische Wirkung der Darlehen.

Insofern ist der Beschäftigungseffekt des Sysmin-Verfahrens (Verhinderung von Entlassungen) im Vergleich zu den oben beschriebenen Erweiterungsprojekten, wo Neueinstellungen nicht immer erforderlich sind, als stärker anzusehen.

Weiterhin positiv zu bewerten ist die Tatsache, daß ein Teil der SysminDarlehen auch zur Fort- und Weiterbildung der Mitarbeiter in den betroffenen AKP-Unternehmen eingesetzt werden kann.

Wie bereits erwähnt liegt eine generelle Schwäche der Sysmin-Regelung in dem Trend, unwillkürlich große Bergbaugesellschaften zu bevorzugen. Zwar sind kleinere oder mittlere Gesellschaften nicht von dem Verfahren ausgeschlossen - das dritte Sysmin-Darlehen wurde Ende des Jahres 1984 an kleinere Zinnabbaubetriebe in Ruanda vergeben -, in praxi jedoch wird die Fazilität primär von Großerzeugern genutzt werden. Dies erklärt sich wie folgt:

so Es werden zusätzliche Kosten vermutet, da auf diese Weise eventuell vorhandene Monopolstellungen verstärkt werden könnten. Persönliche Gespräche, ZCCM, Lusaka; EG-Delegation, Lusaka, Sambia; Vgl. Zambia Consolidated Copper Mines (Hrsg.): Annual Report 1983, a.a.O., S. 4. 
- Die Behörden eines AKP-Staates werden immer bemüht sein, für jene Bereiche günstige Finanzmittel zu organisieren, in denen sie selbst finanziell engagiert sind. Dieses Engagement mag aktiv begründet sein, etwa durch Beteiligungen oder Subventionen, aber auch passiv, aufgrund von Einnahmen wie Steuern, Royalties oder Zöllen.

Insofern ist es naheliegend, daß die AKP-Regierungen der Erhaltung von Großunternehmen - gleich ob unter privater, halbstaatlicher oder staatlicher Führung - stärkeres Interesse entgegenbringen als der einer kleinen - in der Regel privaten - Abbaugesellschaft, die nur eine von vielen ist.

- Der Kenntnisstand über die Existenz der Sysmin-Regelung dürfte bei den Großunternehmen ausgeprägter sein, da deren Beziehungen zu den maßgeblichen Institutionen und Behörden intensiver sind als die der kleinen Abbauunternehmen.

- Den großen Bergbaugesellschaften ist es eher möglich, Personal für die Durchführung des zeitintensiven und aufwendigen Verfahrens abzustellen.

- Unter Umständen sind die kleinen und mittleren Bergbaugesellschaften nicht in der Lage, die mehrjährige Zeitdauer bis zum Eintreffen der durch Sysmin-Darlehen finanzierten Bergbauausrüstungen zu überstehen. Daher müssen sich diese Unternehmen nach anderen, schneller verfügbaren Finanzierungsmöglichkeiten umsehen (z.B. Lieferantenkredite), oder sie müssen ihren Abbaubetrieb einstellen beziehungsweise sie werden aufgekauft.

Als Resultat dieser Gegenüberstellung kann festgehalten werden, daß das Sysmin-Verfahren in entwicklungspolitischer Hinsicht eine deutliche und auch effiziente Unterstützung darstellt. Dies trifft besonders für die neu entwickelte Regelung unter Lome IV zu und gilt sowohl für die Belange der AKP-Staaten als auch für die Interessen der Bergbaugesellschaften. Dennoch erscheint es sinnvoll und möglich zu sein, einige Bereiche der Bergbau-Fazilität weiterhin zu optimieren beziehungsweise vorhandene Ansätze zu vertiefen, so daß ein noch effizienterer Mitteleinsatz gewährleistet wird.

Im Zusammenhang mit den obigen Überlegungen taucht die Frage auf, inwieweit die EG-Entwicklungspolitik mit der EG-Außenhandelspolitik 
gemeinsame Ziele verfolgt. Es ist naheliegend, daß sich hier in einigen Punkten deutliche Gegensätze herausbilden, die nur mittels Kompromissen angenähert werden können.

Als Beispiel für etwaige Zielkonflikte können zwei Zielsetzungen der EGRohstoffpolitik aufgeführt werden. Zum einen ist die Europäische $\mathrm{Ge}$ meinschaft bemüht, den AKP-Staaten bei der Erhaltung und beim Ausbau der Rohstoffproduktion behilflich zu sein und so einen Beitrag zur Entwicklung der Bergbauindustrie beziehungsweise zur Diversifikation der AKP-Industrie zu leisten. Auf der anderen Seite ist die Europäische $\mathrm{Ge}-$ meinschaft bestrebt, sich - im Rahmen der rohstoffpolitischen Bezugsdiversifizierung - auch in anderen Staaten zu engagieren. Derartige Aktivitäten stehen unter Umständen im deutlichen Gegensatz zu den erstgenannten Zielsetzungen und erklären, weshalb Interessenvertreter der EG-Bergbauindustrie gelegentlich die Sysmin-Regelung als eine Art Betriebssubvention bezeichnen und ihr Wettbewerbsverzerrung vorwerfen..$^{51}$

si Vgl. Sames, C.W.: Anaconda, a.a.O., S. 112 f. 



\section{DIE MÖGLICHKEITEN ZUR VERBESSERUNG DER SYSMIN-REGELUNG IN DEN LOMÉ-ABKOMMEN}

\subsection{Ergänzungen zur Sysmin-Regelung}

Wie bisher aufgezeigt wurde, erfüllt das Sysmin-Verfahren, besonders nach seiner Erweiterung in Lome IV, im wesentlichen die Anforderungen, die an die Regelung im Hinblick auf die Unterstützung der Bergbau- und Hüttenindustrie beziehungsweise der von dieser abhängigen AKP-Staaten gestellt werden. Dennoch erscheint es möglich, durch einige Modifikationen im Verfahren weitere Verbesserungen zu realisieren.

Die im nachfolgenden aufgeführten Änderungsmöglichkeiten stellen allerdings nicht immer sofort umsetzbare Alternativen zu den gegenwärtigen Regelungen dar, denn die Durchsetzbarkeit der Modifikationen ist aufgrund der unterschiedlichen Positionen der Vertragspartner nicht immer gewährleistet.

Dem allgemeinen Wunsch der AKP-Staaten nach einer Aufstockung der finanziellen Mittel der Lomé-Abkommen ist bei der Ausstattung des VII. Europäischen Entwicklungsfonds erneut entsprochen worden (s. Tab. 1), auch wenn den AKP-Staaten die Mittelausstattung angesichts der Erweiterung auf 69 AKP-Staaten sowie der erheblichen Schwierigkeiten in den AKP-Staaten als zu gering erscheint. ${ }^{1}$

Generell sei dazu angemerkt, daß nicht allein die Steigerung der zur Verfügung gestellten Mittel, sondern auch die "Qualität" der Vereinbarungen im wesentlichen Maße ausschlaggebend für eine größere Effizienz ist.

Gegen etwaige Versuche der Europäischen Gemeinschaft, eine systematische Einflußnahme auf die Entwicklung der einzelnen Länder auszuüben, verwahren sich die AKP-Staaten nach wie vor entschieden. Gerade aber bei Maßnahmen im Bergbausektor wäre es sinnvoll, projektübergreifende Aktivitäten zu entfalten.

${ }^{1}$ Sefali, M.: "A significant contribution to human understanding and development, a.a.O., S. 8; Schmuck, O.: Lome - Zusammenarbeit auf neuer Grundlage, in: Entwicklung und Zusammenarbeit, 3/90, S. 16 f. 
So kam es besonders in den ersten Jahren der Lomé-Abkommen häufig zu einer Reihe von Einzelaktionen, die bei einer koordinierten Gesamtplanung wesentlich effektiver hätten sein können. Als Beispiel sei hier nochmals die unter Zuhilfenahme von Stabex-Darlehen durchgeführte - vorübergehende - Schließung der Eisenerz-Gruben in Sierra Leone angeführt (s. Kap. 2.5.2).

Des weiteren ist wegen der Vielzahl der Einzelregelungen die Transparenz der Lomé-Abkommen äußerst unbefriedigend.

Besonders die kleineren und weniger entwickelten AKP-Staaten sind in dieser Hinsicht benachteiligt. Thnen steht kein großer Beamtenapparat zur Verfügung, der sich durch die Lomé-Abkommen durcharbeiten und nach Anwendungsmöglichkeiten suchen könnte (s. auch Kap. 4.1: Anfrage Botsuanas, ob Kupfer in die Sysmin-Regelung aufgenommen werden könnte).

Hier wäre von seiten der EG-Kommission Abhilfe zu schaffen, indem EGBerater in den jeweiligen AKP-Bergbauländern die diversen Möglichkeiten der Lomé-Abkommen aufzeigten. Da die in den meisten AKP-Staaten existierenden EG-Außenstellen zur Wahrnehmung dieser Spezialaufgabe nicht geeignet zu sein scheinen, sollten Experten aus Europa diese Mission übernethmen.

Zur Entwicklung eines optimalen Sanierungskonzeptes ist es unerläßlich, daß sowohl der Einsatz der Lomé-Programme koordiniert wird als auch sämtliche Beteiligte integrativ mit in die Planung einbezogen werden. Dabei genügt es nicht, wenn die EG-Berater nur Gespräche mit den jeweiligen Regierungsvertretern und den betroffenen Unternehmen führen. Vielmehr müßten auch Experten supranationaler Institutionen, Repräsentanten von Banken und eventuell auch potentielle Abnehmer der Bergbauerzeugnisse sowie Lieferanten der Bergbauausrüstungen von Anbeginn mit einbezogen werden.

Es versteht sich von selbst, daß ein solches Vorgehen äußerst aufwendig ist und Zeiträume erfordert, die im Gegensatz zu der häufig gewünschten beziehungsweise erforderlichen Soforthilfe stehen. 
Dieser Tatsache ist entgegenzuhalten, daß besonders in der Bergbauindustrie - im Gegensatz zu landwirtschaftlichen Produktionsstätten meistens langfristige Schwierigkeiten auftreten, ${ }^{2}$ so daß ein ausgewogenes Langfristkonzept sinnvoller ist, als mehrere Sofortmaßnahmen, deren Wirkungen jedoch schnell wieder abklingen.

Insofern wäre ein "Round-table-Modell", bei dem sich EG-Berater mit allen Beteiligten zusammensetzen, ein optimales Konzept für die Entwicklung von Langzeitstrategien für Bergbauunternehmen, bei denen sich in der näheren Zukunft gewisse Engpässe abzeichnen. In den Fällen, wo bereits schnelle Aktionen erforderlich sind, wäre dieses Modell je nach Situation zu modifizieren. Durch turnusmäßige Besuche der EG-Experten in den AKP-Bergbaustaaten ließe sich sogar die Notwendigkeit von Sofortmaßnahmen immer weiter reduzieren, da auf diese Weise die mittel- und langfristigen Probleme antizipiert und eventuell auch vermieden werden könnten.

Zusätzlich würde durch die Aktionen - speziell bei mittleren und kleineren Unternehmen - der Bekanntheitsgrad der Lomé-Abkommen weiter gefördert, wodurch gleichzeitig die "Schwellenangst", nämlich von privater Seite auf staatliche Institutionen im eigenen Land sowie auf die Europäische Gemeinschaft zuzugehen, abgebaut wird.

Durch dieses Vorgehen könnte auch die Forderung der Beratenden Versammlung AKP-EWG (seit Lomé III: Paritätische Versammlung), die bereits im Dezember 1982 wirksame Mechanismen für das Funktionieren des Sysmin-Verfahrens verlangte, ${ }^{3}$ im Hinblick auf die Umsetzbarkeit der Sysmin-Regelung erfüllt werden.

\subsection{Modifikationen der Sysmin-Schwellen}

Obwohl seit Lomé 111 neben den explizit erwähnten mineralischen Rohstoffen (15-prozentige Abhängigkeitsschwelle) auch nahezu sämtliche anderen Bergbauerzeugnisse (20-prozentige Abhängigkeitsschwelle) durch

${ }^{2}$ Die sambische Kupfergesellschaft ZCCM wird seit Mitte der siebziger Jahre mit erheblichen Problemen konfrontiert.

3 Vgl. Bersani, G.: Dossier: Die Versammlung AKP-EWG 1964 - 1982, Luxemburg, Februar 1983, S. 142. 
die Bergbaufazilität unterstützt werden können (s. Kap. 2.5.3), erscheint es sinnvoll, die Trennung in mineralische Rohstoffe "erster Klasse" und "zweiter Klasse" aufzuheben und eine für alle Bergbauprodukte gleiche Regelung einzuführen.

Diese Forderung läßt sich zum einen begründen durch die "willkürliche" Erfassung der traditionellen Sysmin-Rohstoffe. Wie erwähnt, wurde der Katalog bei der Etablierung des Sysmin-Verfahrens festgelegt, nachdem in einzelnen AKP-Staaten bei diesen Rohstoffen bereits Schwierigkeiten aufgetreten waren beziehungsweise erwartet wurden (s. Kap. 2.7). Eine Option, weitere Rohstoffe in den Katalog aufzunehmen, besteht seit Lomé IV nicht mehr.

Zum anderen hat sich deutlich herausgestellt, daß auch einzelne Bergbauprojekte für die Volkswirtschaft eines AKP-Staates beziehungsweise für eine Region durchaus positive Effekte bewirken können. Der Erhalt auch von kleineren Bergbauaktivitäten kann aus volkswirtschaftlichen Gesichtspunkten heraus somit auch dann sinnvoll sein, wenn der Gesamtexport von Bergbauerzeugnissen des betroffenen AKP-Staates unter 20 Prozent bleibt.

Darüber hinaus wäre durch eine einheitliche Regelung für alle mineralischen Rohstoffe erreicht, daß die mittlerweile sehr kompliziert gewordene Sysmin-Regelung wieder klarer strukturiert werden könnte, wodurch eventuelle Interpretationsdifferenzen zwischen Antragsteller und Europäischer Gemeinschaft vermieden würden.

Im Zusammenhang mit einer Vereinheitlichung sollte auch die Höhe der Abhängigkeitsschwelle neu überdacht werden. Folgende Varianten der Sysmin-Abhängigkeitsregelung sind denkbar:

a) Anpassung der Abhängigkeitsschwelle für die nicht traditionellen Sysmin-Rohstoffe an diejenigen für die traditionellen Sysmin-Rohstoffe;

b) Reduzierung der Schwelle von "Gesamtexporte" auf "Exporte nach der Gemeinschaft und anderen AKP-Staaten";

c) Genereller Verzicht auf die Abhängigkeitsschwelle. 
ad a: Eine solche Anpassung ließe sich mit dem geringen Anteil der selteneren mineralischen Rohstoffe am Gesamthandel - im Gegensatz zu den Massenrohstoffen (Kupfer, Eisenerz) - begründen (s. Tab. 12). Demzufolge könnten auch solche AKP-Staaten von der SysminRegelung profitieren, die Rohstoffe abbauen, welche - wie Niob, Titan, Vanadium - nur in geringen Mengen auftreten. Da diese Bergbauprodukte von strategischer Bedeutung sind, würde auch dem starken Interesse der Europäischen Gemeinschaft an der Lieferung dieser Rohstoffe entsprochen. Kritiker des Sysmin-Verfahrens, die behaupten, in der Sysmin-Regelung seien im wesentlichen nur die Rohstoffsicherungsaspekte der Europäischen Gemeinschaft berücksichtigt, würden durch diese Modifikation allerdings neue Argumente erhalten.

ad b: Da die AKP-Staaten in der Regel den größten Teil ihrer Bergbauerzeugnisse nach der Europäischen Gemeinschaft exportieren, ${ }^{4}$ kann sich, falls die Gesamtexportbilanz anders strukturiert ist, bei ausschließlicher Betrachtung der Exporte nach der Europäischen Gemeinschaft der Prozentsatz der mineralischen Rohstoffe erhöhen, wodurch die AKP-Bergbauexporte schneller die erforderliche Abhängigkeitsschwelle erreichen würden.

Die Reduzierung der Abhängigkeitsschwelle vom Gesamtexport auf Exporte nach der Gemeinschaft hätte auch positive Auswirkungen auf die Rohstoffversorgungssicherheit der Europäischen Gemeinschaft, da sich die AKP-Rohstoffproduzenten dann zusätzlich bemühen, Bergbauerzeugnisse nach der Gemeinschaft zu liefern. Besonders bei den mineralischen Rohstoffen mit hoher EG-Importabhängigkeit wäre dies ein für die Europäische Gemeinschaft wünschenswerter Effekt. Den unter a) erwähnten, kritischen Stimmen wäre mit dieser Version jedoch ebenfalls Vorschub geleistet.

ad c: Bei einem ersatzlosen Verzicht auf die Abhängigkeitsschwelle würde deutlich der entwicklungspolitische Aspekt der Sysmin-Regelung hervorgekehrt. Eine Benachteiligung der AKP-Staaten, die nur geringere Mengen von mineralischen Rohstoffen produzieren, unterbliebe, es könnten auch kleinere Bergbauaktivitäten Mittel aus dem Sysmin-Fonds erhalten.

${ }^{4}$ Statistisches Amt der EG (Hrsg.): EC-ACP-Trade, a.a.O., S. 185 f. 
Da bekanntlich auch schon kleinere Exporterlösschwankungen in unflexiblen Wirtschaftsordnungen erhebliche Nachteile verursachen können, leiden häufig selbst AKP-Staaten mit einer wenig ausgeprägten Bergbauindustrie unter den Konsequenzen von Exporterlösschwankungen bei Bergbauerzeugnissen.

Bei einem ersatzlosen Streichen der Abhängigkeitsschwelle besteht allerdings die Gefahr, daß der finanzielle Rahmen der Sysmin-Fazilität aufgrund der stärkeren Inanspruchnahme schnell erschöpft sein könnte. Das Aufstellen weiterer Kriterien - wie die Beschränkung der Mittelvergabe auf gewisse Prozentsätze pro Jahr und AKP-Staat oder die Festlegung von Prioritätsstufen (Kleinbergbau vor Großprojekten etc.) - könnte diese Tatsache verhindern. Darüber hinaus kann durch die Hinzuziehung weiterer Mittel aus dem Lomé-Abkommen (Investitionsfonds, Umweltfonds etc.) eine Streckung der Sysmin-Mittel erreicht werden.

Die seit Lomé III bestehende Erfassung auch von AKP-Bergbaustaaten, die nicht nur von einem mineralischen Rohstoff abhängig sind, war ein Schritt in die richtige Richtung. Jedoch sind nach dieser Regelung zur Zeit nur 4 weitere AKP-Staaten berechtigt, Anträge für Mittel aus der Sysmin-Fazilităt zu stellen: Botsuana, Namibia, Niger und Simbabwe. ${ }^{5}$ AKP-Staaten, deren Bergbauindustrie nur einen geringen Anteil der Ausfuhrerlöse erwirtschaftet oder die ausschließlich Lagerstätten mit seltenen und Edelrohstoffen (z.B. Diamanten) aufweisen, werden nach wie vor durch das Sysmin-Reglement keine Unterstützung erfahren.

Auch der Kleinbergbau wird bei den jetzigen Sysmin-Modalitäten nur gelegentlich Nutznießer des Verfahrens sein können, da Kleinbetriebe nur selten genügend mineralische Rohstoffe produzieren, um dem AKP-Staat zu den erforderlichen Sysmin-Schwellen zu verhelfen. Die Bereitstellung von Sysmin-Mitteln an den handwerklich betriebenen Zinnbergbau in Ruanda ${ }^{6}$ wird daher eine Ausnahme bleiben.

Diese Tatsache ist bedauerlich, da eine Unterstützung des Klein- und mittleren Bergbaues aus entwicklungs- und rohstoffpolitischer Sicht sinnvoll und effizient sein kann.

5 Persönliches Gespräch, Kommission der EG, Brüssel.

6 Vgl. Kommission der EG (Hrsg.): Mitteilungen an die Presse, IP (84) 347, a.a.O. 
Entwicklungspolitisch vorteilhaft ist, daß diese Bergwerke weniger kostenintensiv sind als große Abbauunternehmen und eine größere Personalintensivität aufweisen. Der verhältnismäßig starke Einsatz von einheimischen Führungskräften und die flexible Anpassung an Markterfordernisse gehört ebenfalls zu den positiven Merkmalen dieser Kleinbetriebe.

Da mineralische Rohstoffe, die für die Europäische Gemeinschaft von strategischer Bedeutung sind (s. Tab. 9), häufig in kleineren und mittleren Bergwerken abgebaut werden, sind diese auch unter rohstoffpolitischen Aspekten zu betrachten: Durch eine gezielte Unterstuitzung der kleinen und mittleren Abbaubetriebe könnte die Versorgung der Europäischen Gemeinschaft mit strategischen Rohstoffen auf eine breitere Basis abgestellt werden.

Insofern wird an dieser Stelle dringend dafür plädiert, bei einer erneuten Überarbeitung des Sysmin-Verfahrens neben einer einheitlichen Bemessungsgrundlage für alle mineralischen Rohstoffe gemäß Punkt c) auf eine Abhängigkeitsschwelle völlig zu verzichten. Angesichts der seit Lomé IV vorgeschriebenen, umfangreichen Gutachten, die zur Begründung des Antrags auf Sysmin-Mittel zu erstellen sind (Lomé IV: Art. 216 und 217), erscheint ein Mißbrauch der Sysmin-Fazilität sehr weit eingeschränkt zu sein. Ein wesentlich erhöhter Mittelbedarf läßt sich - wie in Punkt c) aufgefuiht - durch zu vereinbarende Restriktionen vermeiden. Die in der Sysmin-Präambel enthaltene Klausel "..., für deren Wirtschaft der Bergbau eine wichtige Rolle spielt ..." (Lomé IV: Art. 214) sollte ergänzt werden: "... für deren Wirtschaft, Infrastruktur oder Bevölkerung der Bergbau insgesamt oder regional eine nicht unerhebliche Rolle spielt ...".

\subsection{Die Sysmin-Erweiterung auf Produkte aus der Weiterverarbei- tung}

Bei der Konzipierung der Sysmin-Regelung Ende der siebziger Jahre stand die Idee im Vordergrund, die Produktions- und Exportkapazitäten der AKP-Bergbauindustrie zu unterstützen, falls diese unter größeren Störungen leiden sollten. Wiederherstellung, Unterhaltung und Modernisierung 
der Produktionsanlagen sollten bei dem Sysmin-Verfahren Priorität besitzen (s. Kap. 2.5.3). ${ }^{7}$

Deutlich wird in den jeweiligen Sysmin-Präambeln auf den Zusammenhang zwischen dem Sysmin-Verfahren und entwicklungspolitischen Aspekten hingewiesen: "Die Zielsetzung des Systems besteht darin, einen Beitrag zur Schaffung einer stabileren oder sogar erweiterten Grundlage für die Entwicklung der AKP-Staaten zu leisten ..." (Lome IV: Art. 214 Abs. 2; Lomé III. Art. 176; Lomé II. Art. 49) (s. auch Anhang 4).

Bei den ersten Anwendungsfällen zeigte sich, daß die Sysmin-Mittel bestenfalls in der Lage waren, den Status quo ante wieder herzustellen; zIkunftsweisende Projekte, die entwicklungspolitische Maßnahmen unterstützen könnten, ließen sich nur in geringem Umfange realisieren.

Speziell die Bergbauindustrie ist als extraktiver Sektor eher darauf angewiesen, die Erschöpfbarkeit ihrer Lagerstätten zu antipizieren und rechtzeitig Anschlußprojekte vorzubereiten.

Das gleiche gilt im wesentlich ausgeprägteren Maße für die Regierungen von Bergbaustaaten, da im Falle von Bergwerksschließungen zusätzlich zum Rückgang der Staatseinnahmen auch noch sozialpolitische Probleme (u.a. Arbeitslosigkeit) hinzukommen.

Bisher jedoch waren in vielen Entwicklungsländern, dies wurde auch bei den empirischen Erhebungen in Sambia deutlich, keine großen Erfolge beim Aufbau anderer Industrien mit Hilfe der Exporterlöse aus Bergbauprodukten erzielt worden. In Sambia konnte selbst die Weiterverarbeitung nicht im Maße des Möglichen ausgebaut werden. Auch die bisherigen Lomé-Abkommen haben nicht viel dazu beitragen können. Wie sonst erklärt es sich, daß die Afrikanische Entwicklungsbank für Sambia mittels einer internationalen Ausschreibung Angebote über unter anderem kupferummantelte Erdungsstäbe, PVC-isolierte Kabel, blanke Kupferleiter anfordert, also Produkte für ein kupferproduzierendes Land par excellence in Auftrag gibt, die sich ohne großes Know-how und komplizierte Technik auch in einem Entwicklungsland herstellen lassen?

7 Vgl. O.V.: Negotiations update, in: The Courier, Nr. 85, Mai - Juni 1984, S. gelb III.

$8 \mathrm{Vgl}$. o.V.: Ausschreibungen, in: Nachricht für den Außenhandel, 17. Oktober 1985, S. 7. 
Auch nach dem Sysmin-Reglement in Lomé IV ist die Förderung der Weiterverarbeitung explizit nicht vorgesehen. Die vorgesehene Unterstützung von Diversifizierungsprogrammen oder die Erweiterung der Grundlagen für ihr Wirtschaftswachstum (Lome IV: Art. 214 Abs. 2) schließt die weiterverarbeitende Industrie nicht unbedingt mit ein (s. Kap. 4.4).

Der Vorschlag der AKP-Vertreter, das Sysmin-Verfahren solle im Hinblick auf die Abhängigkeits- und Auslöseschwelle nicht nur mineralische Rohstoffe, ${ }^{9}$ sondern auch Halb- und Fertigprodukte erfassen, ${ }^{10}$ erscheint zunächst verständlich. Gleichwohl läßt sich die mangelnde Effizienz einer solchen Regelung sehr schnell darlegen:

- Die Schwankungen der Ausfuhrerlöse sind bei Halb- und Fertigprodukten nie so extrem wie bei Rohstoffen, so daß die AKP-Staaten auch aufgrund dieser Kriterien nicht vorzeitiger die erforderlichen Schwellen erreichen, um die Sysmin-Mittel beantragen zu können.

- Die nicht allzu üppig ausgestaltete Sysmin-Fazilität würde in größerem Umfange auch für andere $Z$ wecke eingesetzt werden können (z.B. Unterstützung der weiterverarbeitenden Industrie), so daß für den Bergbau und die Grundstoffindustrie somit weniger Mittel zur Verfügung stünden.

- Die Etablierung von weiterverarbeitender Industrie und anderen Wirtschaftsbereichen würde auch durch eine solche Sysmin-Erweiterung nicht gezielt gefördert werden (s. Kap. 4.4), dafür stehen im Rahmen der Lomé-Abkommen andere Instrumente zur Verfügung.

Noch entscheidender für den effizienten Einsatz von Sysmin-Mitteln jedoch ist die Berücksichtigung des gesamten Produktionsumfeldes in dem betroffenen AKP-Staat. Der Fall Ruanda mag diese Aussage verdeutlichen.

Im Jahre 1985 erhielt der AKP-Staat Ruanda - wie erwähnt - zur Unterstützung seines überwiegend handwerklich betriebenen Zinnbergbaues Darlehen aus der Sysmin-Fazilität. Dieser prinzipiell sinnvollen Maßnahme zur Stärkung des Kleinbergbaues war aber insofern nur geringer Erfolg beschieden, da die einzige Zinnhütte in Ruanda, Somirwa kurz

9 Definition s. Abschnitt 4.6.

$10 \mathrm{Vgl}$. O.V.: Negotiations update, a.a.O., S. gelb III. 
nach dem Sysmin-Transfer im Oktober 1985 Konkurs anmelden mußte, ${ }^{11}$ und die Kleinproduzenten somit nicht mehr auf einen inländischen $\mathrm{Ab}$ nehmer für ihre Zinnerze zurückgreifen konnten. ${ }^{12}$

Obwohl in diesem Falle der Sysmin-Regelung kein unmittelbares Versagen anzulasten ist, wird deutlich, daß punktuelle Aktionen leicht zum Scheitern verurteilt sind, wenn das weitere Umfeld der Produktion mineralischer Rohstoffe nicht mitberücksichtigt wird.

Insofern wird an dieser Stelle die Forderung erhoben, zunächst - wie im Kapitel 6.2 ausgefuihrt - auf die Abhängigkeitsschwelle zu verzichten, den Sysmin-Katalog einheitlich auf sämtliche mineralischen Rohstoffe - einschließlich Erdöl und Erdgas - auszudehnen und darüber hinaus die Möglichkeit zu schaffen, daß nach sorgfältiger Prüfung und Abwägung die Sysmin-Mittel auch dann in der weiterverarbeitenden Industrie eingesetzt werden können, wenn es sich nicht um Diversifizierungsprojekte handelt. So wäre es unter Umständen möglich, daß ein zum Beispiel international nicht wettbewerbsfähiges Bergbauunternehmen seine Erzeugnisse im Inland absetzt, wo aufgrund der Ausnutzung sonstiger Wettbewerbsvorteile (z.B. Lohnniveau) Grundstoffe beziehungsweise Produkte hergestellt werden, die auf dem Weltmarkt konkurrenzfähig sind.

Weiterhin wird an dieser Stelle dringend empfohlen, Regelungen zu schaffen, wonach Mittel aus der Sysmin-Fazilität unabhängig von jeglichen Kriterien, einschließlich Wirtschaftlichkeitsbetrachtungen für Umweltschutzmaßnahmen im Bergbau eingesetzt werden können. Zur Zeit können und werden Sysmin-Mittel bereits zum Teil für Vorhaben im Umweltschutz eingesetzt (s. Kap. 4.2), dies jedoch nur, falls die Sysmin-Bedingungen erfüllt sind. Da jedoch Maßnahmen im Umweltschutz nur in seltenen Fällen nach wirtschaftlichen Kriterien bewertet werden können und in einem Unternehmen, welches sich in Schwierigkeiten befindet, in der Regel zunächst andere Maßnahmen als für den Umweltschutz ergriffen werden, ist die obige Empfehlung nur eine konsequente Weiterentwicklung der in Lomé IV zum ersten Male unverbindlichen Absichtserklärung der Vertragspartner, im Umweltschutz eng kooperieren zu wollen (Lomé IV: Art. 33 ff.). Dieser Vorschlag gewinnt zusätzlich Gewicht durch die

$11 \mathrm{Vgl}$. O.v.: Dissolution de la Somirwa, in: Marchés tropicaux, 8. November 1985, S. 2773.

12 Persönliches Gespräch, Bundesministerium für Wirtschaft, Bonn, Januar 1986. 
Tatsache, daß die Sysmin-Regelung erklärtermaßen entwicklungspolitische Ziele hat und daß der Bergbau zu den umweltrelevanten Industrien zählt.

Zur Vermeidung einer schnellen Erschöpfung der Sysmin-Mittel aufgrund der zu erwartenden Vielzahl von Anträgen, sollte ein zu definierender Anteil (20 Prozent) des Sysmin-Fonds für diese Zwecke reserviert werden. Anträge, die bis zu einem Stichtag eingehen, werden nach Dringlichkeit der Umweltmaßnahme genehmigt. Die aufgrund der Etat-Überschreitung nicht berücksichtigten Anträge können dann im nächsten Jahr erneut vorgelegt werden.

\subsection{Die Modifikation der Zahlungsmodalitäten}

Die Höhe des Sysmin-Fonds war - wie erwähnt - bereits bei der Unterzeichnung des Lomé-Il-Abkommens von den AKP-Staaten als zu niedrig angesehen worden. ${ }^{13}$ Die EG-Kommission sowie die EG-Mitgliedstaaten gehen jedoch davon aus, daß die Sysmin-Fazilität nicht die einzige Finanzierungsquelle ist, auf welche die wirtschaftlichen, kurzfristig in finanzielle Engpässe geratenen AKP-Bergbauindustrien zurückgreifen können. In der Tat wäre der Sysmin-Etat deutlich unterdimensioniert, wenn es nicht noch andere Fazilitäten gäbe, um die AKP-Staaten im Bergbausektor zu unterstützen. Allein Sambia hätte zur Sanierung seines Kupferbergbaues den größten Teil der Sysmin-Mittel aus Lomé II und Lomé III benötigt.

Vielmehr gehen die Europäer davon aus, daß in Rehabilitationsprogrammen sämtliche Möglichkeiten, die sowohl durch die Lomé-Abkommen als auch durch andere supranationale, staatliche und private Kreditgeber eingeräumt werden, auszuschöpfen sind. Zwar verlangt ein derartiges Konzept die generelle Akzeptanz des Rehabilitationsplanes durch alle Beteiligten, wodurch sich die Mittelvergabe als wesentlich zeitaufwendiger erweist als bei einem Direktdarlehen. ${ }^{14}$ Vom entwicklungspoli-

${ }^{13}$ Vgl. Twitchett, C.C.: Lomé II: die Enttäuschungen ..., a.a.O., S. 85; St. John, B.: "The groundwork ..., a.a.O., S. 5.

14 Selbst Ende 1988 waren erst 90 Prozent der Sysmin-Fazilität aus Lomé II und 16 Prozent aus Lomé III genehmigt worden. Vgl. Kommission der EG (Hrsg.): Lomé III - Mid term review 1986 - 1988, a.a.O. S. $17 \mathrm{f}$. 
tischen Standpunkt aus betrachtet erscheint eine solche Vorgehensweise jedoch sinnvoller zu sein, da so nicht nur singuläre, sondern umfassende Lösungen erzielt werden.

Im Hinblick auf die Lomé-Abkommen beispielsweise setzt die Europäische Investitionsbank (EIB) einen Großteil ihrer Mittel, die im Rahmen von Lome zur Verfügung stehen, in Form von zinsbegünstigten Darlehen, Risikokapital und Teildarlehen aus Globaldarlehen unter Zwischenschaltung von AKP-Entwicklungsbanken für Bergbau-, Energie- und Industrialisierungsprojekte in den AKP-Staaten ein. ${ }^{15}$ Des weiteren werden auch Mittel des Europäischen Entwicklungsfonds - über die Sysmin-Fazilität hinaus - für Vorhaben im Bergbausektor vergeben (Lomé IV: Art. 99 bis 104). Neben den bereits aufgeführten Änderungsvorschlägen bietet sich die Modifikation der Rückzahlungsmodalitäten als besonders sinnvolle Lösung an, weil es im Hinblick auf die anderen Kreditilgungsverpflichtungen so zu einer Entzerrung der Schuldendienstleistungen kommen kann.

Im Zusammenhang mit der Optimierung der Zahlungsmodalitäten ist zunächst noch einmal auf die seit Lomé IV geltende Regelung zur Vergabe der Sysmin-Tranchen einzugehen. Wie auch bei dem Stabex-Verfahren wird nunmehr die Finanzhilfe aus der Sysmin-Fazilität dem AKP-Staat nicht mehr als Sonderdarlehen, sondern als nichtrückzahlbarer Zuschuß gewährt. ${ }^{16}$ Dieser Zuschuß wird dann wie auch bei den bisherigen Regelungen als Darlehen mit Sonderkonditionen an die begünstigten AKPBergbauunternehmen vergeben (s. Kap. 2.5.3).

Obwohl diese Praxis aus entwicklungspolitischen Gesichtspunkten durchaus zu begrüßen ist, erscheint die Anwendung einer solchen generellen Bezuschussung nicht für alle AKP-Staaten unbedingt erstrebenswert $z$ sein. Dies trifft besonders bei jenen AKP-Staaten zu, die bereits durch die Existenz einer hochentwickelten Bergbauindustrie nicht mehr zu den am wenigsten entwickelten Ländern gehören, und die in der Lage sein sollten, durch hinreichende Exporterlöse sowie eine solide Finanzplanung derartige Sonderdarlehen zurückzuzahlen. Solche AKP-Staaten könnten sich auf diese Weise langfristig auch eine gewisse Reputation und Kreditwürdigkeit aufbauen, ein Umstand der besonders wichtig ist, wenn diese AKP-

15 Im Jahre 1982 nahezu 90 Prozent. Vgl. Kommission der EG (Hrsg.): Bericht ..., a.a.O., S. 9 f.

$16 \mathrm{Vgl}$. Bundesrat (Hrsg.): Entwurf eines Gesetzes zu dem Vierten AKPEWG-Abkommen ..., a.a.O., S. 196. 
Staaten das Image eines Entwicklungslandes, welches seine Schuldenprobleme nicht beherrscht, mittel- und langfristig abschütteln wollen.

Daher wird an dieser Stelle empfohlen, die Sysmin-Mittel - soweit dies bei den Verhandlungspartnern durchsetzbar ist - wieder als Sonderdarlehen zu vergeben. Ausnahmen in Form von Zuschüssen sollten allein bei LDC und bei Umweltschutz-Vorhaben im Bergbau (s. auch Kap. 5.3) möglich sein. Dadurch würde neben dem oben beschriebenen möglichen Aufbau einer gewissen Kreditwürdigkeit erreicht, daß die knapp bemessene Fazilität nach einer absehbaren Zeit wieder einen Mittelzufluß erhielte, der im Sinne eines "revolving fund" für neue Vorhaben zur Verfügung stünde.

Um dennoch Schwierigkeiten bei der späteren Rückzahlung der SysminTranchen zu vermeiden beziehungsweise zu reduzieren, sind neue Formen der Rückzahlungsmodalitäten zu entwickeln.

Daß Schwierigkeiten bei der Aufbringung von Mitteln zur Rückzahlung der Sysmin-Tranchen - auch bei einer soliden Finanzplanung - eintreten können, zeigen die Erfahrungen aus der Vergangenheit. Dies trifft sowohl für die AKP-Staaten zu als auch für die Bergbauunternehmen selbst und läßt sich wie folgt erklären:

Die kapitalintensiven, devisenabhängigen Bergbauindustrien kürzen bei finanziellen Engpässen ihre Ausgaben in der Regel wie folgt:

(1) Erweiterungsinvestitionen;

(2) Ersatzteile, Betriebs- und Hilfsstoffe (Lagerhaltung)

(3) Prospektions- und Explorationsinvestitionen;

(4) Ersatzteile, Betriebs- und Hilfsstoffe (Beschaffung);

(5) Personalbestand.

Während sich die reduzierte Lagerhaltung beziehungsweise der Mangel an Ersatz-, Betriebs- und Hilfsstoffen in operativen Zeiträumen auswirkt, machen sich die anderen Kürzungen erst in taktischen und verstärkt in strategischen Zeiträumen bemerkbar. Werden nach einem Liquiditätsengpaß externe Mittel bereitgestellt, werden diese in der Regel zunächst zur unmittelbaren Wiederherstellung der Betriebsbereitschaft eingesetzt. Dies wurde auch durch die Erhebungen in Sambia deutlich. Dort sind die ersten Darlehen aus der Sysmin-Fazilität hauptsächlich für 
Ersatzteile beziehungsweise Ersatz- und Ergänzungsinvestitionen verwandt worden (s. Kap. 4.3).

Wenn die angeschafften Ersatzteile aufgebraucht sind oder kurzfristig weitere Investitionen zur "Aufrechterhaltung der Produktionskapazität" getätigt werden müssen, stellt sich die Finanzierungsfrage erneut. Denn die zur Verfügung gestellten Darlehen sind nach kurzer Zeit aufgezehrt, und es fehlen wiederum Mittel, um die Produktion im erforderlichen Maße aufrecht zu erhalten. Der Zustand wie vor der Kreditvergabe ist wieder erreicht, da mit Hilfe der Sysmin-Darlehen - abgesehen von einer weiteren Verbindlichkeit - keine grundlegenden Veränderungen eingeleitet werden konnten. Verstärkt wird diese Tatsache durch die langjährige Laufzeit der Sysmin-Dariehen. Von den Unternehmen wird eine langfristige Verschuldung (in der Regel über 10 Jahre) eingegangen, es werden jedoch häufig keine langlebigen Investitionsgüter angeschafft.

Ein im Zusammenhang mit der langen Kreditlaufzeit der Sysmin-Darlehen zusätzliches Problem kann aus der Nichterneuerbarkeit der mineralischen Rohstoffe erwachsen.

Ist es überhaupt ratsam, einem AKP-Bergbauunternehmen Darlehen mit Laufzeiten zur Verfügung zu stellen, welche den Abbauzeitraum der Bergbaureserven - wie im Falle von Abbaubetrieben der Zambia Consolidated Copper Mines ${ }^{17}$ - nach dem gegenwärtigen Kenntnisstand überschreiten? Wird ein solchermaßen begünstigtes Unternehmen überhaupt in der Lage sein, den Schuldendienst zu leisten?

Im Hinblick auf diese Fragestellung liegt die Überlegung nahe, den AKPBergbauunternehmen Möglichkeiten einzuräumen, die Sysmin-Darlehen in anderer Form als durch finanzielle Rückzahlung zu tilgen.

Eine solche Alternative bietet das im internationalen Warentausch - besonders mit den ehemaligen Staatshandelsländern - häufig praktizierte Verfahren des Kompensationshandels. Dies setzt jedoch voraus, daß das einzelne Bergbauunternehmen direkter Kreditnehmer bei der Europäischen

17 Persönliche Gespräche, ZCCM, Lusaka, Sambia und Kommission der EG, Brüssel; Eigene Berechnungen; Vgl. Freeman, P.V.: Mining in Zambia today, a.a.O, S. 1; O.V.: Der Kupfergürtel Lusakas verliert schnell an Glanz, in: Handelsbatt, 23. Februar 1988; Zambia Consolidated Copper Mines (Hrsg.): Annual Report 1988, S. 43 f. 
Gemeinschaft wäre und seine Darlehen auch direkt an den EEF zurückzuzahlen hätte. Eine derartige Regelung unterliefe zwar das bisherige Prinzip der EG-Kommission, EEF-Mittel nur an staatliche Institutionen zu gewähren, ${ }^{18}$ die Vorteile sind jedoch offensichtlich. Zum einen wird das Verschuldungspotential der ohnehin schon stark belasteten AKP-Staaten nicht weiter strapaziert beziehungsweise das Potential kann für andere Projekte, zum Beispiel weniger rentable Entwicklungsvorhaben eingesetzt werden. Zum anderen hat die begünstigte Bergbaugesellschaft die Möglichkeit, mit Hilfe ihrer Erzeugnisse die Darlehen zurückzuführen. Darüber hinaus würde durch eine derartige Modifikation der bisherigen Praxis kleineren Bergbauunternehmen sowie Gesellschaften, die bisher noch keine Handelsbeziehungen zur europäischen Industrie unterhalten, Unterstützung zuteil.

Neben der Form des Counterpurchase ${ }^{19}$ oder des Buyback ${ }^{20}$ bietet sich für das Sysmin-Verfahren eine weitere Lösung an: Bei dieser, bisher noch nicht praktizierten Variante des Kompensationshandels würde das jeweilige AKP-Bergbauunternehmen (zusätzliche) Rohstoffe nach der Europäischen Gemeinschaft liefern. Der Gegenwert für die Bergbauerzeugnisse würde - nach Abzug der Kosten für Transport, Aufbereitung etc. entsprechend dem net-back-Verfahren - auf ein Treuhandkonto bei der Europäischen Gemeinschaft eingezahlt werden. Dort würde eine Aufteilung des Betrages vorgenommen werden in Erlöse für das produzierende Bergbauunternehmen sowie Tilgungsraten für den Europäischen Entwicklungsfonds (EEF). Durch einen zu vereinbarenden Prozentfaktor, der beispielsweise an den Cash flow der AKP-Bergbaugesellschaft gekoppelt sein kann, wäre es möglich, auf die jeweils aktuelle Finanzlage des einzelnen Unternehmens einzugehen.

18 Persönliches Gespräch, Kommission der EG, Generaldirektion Entwicklung, Brüssel.

19 Verpflichtung eines ausländischen Lieferanten (EG), inländische Produkte (AKP) zu exportieren; Persönliches Gespräch, Metallgesellschaft.

20 Verpflichtung eines ausländischen Lieferanten (EG), inländische Produkte (AKP), die auf von ihm gelieferten Anlagen erzeugt wurden, abzunehmen; Persönliches Gespräch, Metallgesellschaft. 
Ein Modell könnte wie folgt aussehen:

Übersteigt der Cash flow des letzten Vierteljahres x Prozent der Betriebsleistung, werden y Prozent des Gegenwertes der Kompensationslieferung als Schuldendienst an den EEF gezahlt, der Rest verbleibt bei den AKPProduzenten (s. Tab. 24).

Tabelle 24: Modell für variable Schuldendienstleistungen an den Europäischen Entwicklungsfonds (EEF)

\begin{tabular}{|c|c|c|}
\hline $\begin{array}{l}\text { Cash flow } \mathbf{x} \\
\text { (in \% der Betr.- } \\
\text { Leistung) }\end{array}$ & $\begin{array}{c}\text { Anteil an EEF } \\
y(\text { in } \%)\end{array}$ & $\begin{array}{l}\text { Anteil an AKP-Unter- } \\
\text { nehmen } 100-y \text { (in \%) }\end{array}$ \\
\hline $\begin{array}{lr}\text { unter } & 5 \\
& 7 \\
& 10 \\
& 15 \\
& 20 \\
& 25\end{array}$ & $\begin{array}{l}20 \\
25 \\
40 \\
50 \\
70 \\
90\end{array}$ & $\begin{array}{l}80 \\
75 \\
60 \\
50 \\
30 \\
10\end{array}$ \\
\hline
\end{tabular}

Anm.: Erläuterung im Text

Quelle: Eigener Entwurf.

Dieses Modell wäre sowohl auf die einzelnen Bergbauunternehmen als auch auf die jeweils zu veräußernden Rohstoffe abzustimmen.

Sollten sich auf dem Europäischen Markt Schwierigkeiten mit dem Absatz zusätzlicher Mengen einstellen, müßte sich die Kompensationsvereinbarung auf das Quantum beschränken, welches an die Europäische Gemeinschaft ausgeführt wird. Diese Einschränkung wäre nicht nötig, wenn die EG-Kommission bereit wäre, für zusätzlich nach der Europäischen Gemeinschaft eingeführte Bergbauerzeugnisse eine Händlerfunktion zu übernehmen. Neben einem Verkauf außerhalb der Europäischen Gemeinschaft könnten auch EG-eigene Ausgleichlager aufgebaut werden, vorausgesetzt, es besteht keine Bereitschaft in den einzelnen EG-Mitgliedstaaten, zur Aufstockung ihrer nationalen strategischen Reserve gewisse Bestände zu übernehmen. 
Das Verfahren, den "direkten Schuldendienst" durch das Bergbauunternehmen an den EEF nicht an starre Rückzahlungsvereinbarungen zu koppeln, weist folgende Vorteile auf:

Das AKP-Bergbauunternehmen

- kann mittels seiner Bergbauerzeugnisse die Zins- und Tilgungszahlungen leisten, es werden keine Devisen benötigt;

- kann die Tilgungen derart variieren, daß bei hohen Erlösen größere Raten- bzw. Kompensationszahlungen an den EEF geleistet werden, bei niedrigeren Erlösen geringere Schuldendienstleistungen realisiert werden;

- kann, da Tilgungsfreiräume nicht eingehalten werden müssen, bereits frühzeitig mit den flexiblen Schuldendienstzahlungen beginnen; dadurch ist die Gefahr, daß nach der Erschöpfung der Rohstoffvorkommen noch Verbindlichkeiten bestehen, geringer als bei starren Rückzahlungsverpflichtungen;

- kann bei einem etwaigen Kompensationshandel die Vermarktung der Rohstoffe einem europäischen Spezialunternehmen übertragen.

Die EG-Kommission

- erzielt mittels der angepaßten Schuldendienstraten in der Handelsbilanz der AKP-Bergbaustaaten eine Art Erlösstabilisierung, da bei geringeren Erlösen weniger, bei höheren Erlösen mehr an den EEF abzuführen ist;

- verringert durch den Verzicht auf starre Rückzahlungsvereinbarungen und die Akzeptanz von Kompensationsgeschäften die Probleme des Schuldendienstes bei den einzelnen AKP-Bergbauunternehmen;

- unterstützt das AKP-Bergbauunternehmen gegebenenfalls bei der Vermarktung seiner Rohstoffe;

- hat eine zusätzliche Rohstoffsicherungskomponente realisiert, da die AKP-Bergbauunternehmen nunmehr ein zusätzliches Interesse haben, mineralische Rohstoffe nach der Europäischen Gemeinschaft zu liefern. 
Die Nachteile dieser Verfahren sind besonders bei den "kompensatorischen Schuldendienstleistungen" offensichtlich:

Da dem betroffenen Bergbauunternehmen eine Art von Abnahmegarantie gewährt würde, könnte dies sowohl zu Wettbewerbsverzerrungen gegenüber den nicht begünstigten Marktteilnehmern führen als auch Rohstoffüberschüsse in der Europäischen Gemeinschaft hervorrufen. Des weiteren wären bei zusätzlichen Lieferungen nach der Europäischen Gemeinschaft Mittel des EEF für die Errichtung und Unterhaltung von kostenintensiven Zwischenlagern erforderlich, da die EG-Kommission nicht bereit sein kann, einen eventuellen Rückgang der Produzentenpreise - bedingt durch ein von ihr konzipiertes Verfahren - in Kauf zu nehmen.

Insofern erscheint eine Vermarktung von zusätzlichen AKP-Rohstofflieferungen durch die EG-Kommission in der ersten Phase der Sysmin-Optimierung sehr aufwendig. Daher sollte die Regelung der Kompensationszahlung zunächst auf die bisher üblichen Rohstofftransfers aus den AKP-Staaten beschränkt werden, sofern nicht noch andere Ziele der EGPolitik (z.B. Bildung einer strategischen EG-Reserve) durch derartige Verfahren zu erreichen sind.

Eine weitere Alternative zu den gegenwärtigen Sysmin-Zahlungsmodalitäten ist in einer Beteiligung europäischer Kapitalgeber - nicht nur die rohstoffverarbeitenden Industrien - an den betroffenen Bergbauunternehmen zu sehen. Vorstellbar sind Joint Ventures und Eigenkapitalbeteiligungen in Form von Aktienübernahmen oder durch Kapitalerhöhungen.

Brauchbare Konzepte für eine Beteiligung von Unternehmen aus Industrieländern an Gesellschaften in Entwicklungsländern sind bereits entwickelt worden, so daß auf deren Grundlage ein praktikables Verfahren konzipiert werden könnte. Besonders geeignet erscheint eine Methode zu sein, die sich an den Grundsätzen des "Guaranteed Recovery of Investment Principal"-Programms (GRIP), einem neuen Finanzierungsinstrument der International Finance Corporation (IFC) orientiert. Das wesentliche Charakteristikum von GRIP ist - ähnlich wie bei der im Jahre 1986 in Washington gegründeten Multilateral Investment Guarantee Agency die Übernahme des Verlustrisikos durch die IFC. ${ }^{21}$

21 Vgl. Braun, H.-G.: Schulden-Swap und GRIP-Instrumente zur Entschärfung der internationalen Verschuldungskrise?, in: ifo-schnelldienst $1-2 / 87,13$. Januar 1987, S. 5. 
Berücksichtigt man die Belange der EG-Unternehmen, die eine Beteiligung an AKP-Bergbaugesellschaften oder eine Joint-Venture-Gründung anstreben, könnte in Anlehnung an GRIP folgendes Verfahren vereinbart werden:

EG-Investoren stellen der EG-Kommission beziehungsweise einer neu zu gründenden EG-Beteiligungsgesellschaft (EG-BGes) den Beteiligungsbetrag in ECU zur Verfügung und erhalten dafür von der EG-BGes einen ECU-Schuldschein. Mit dem Kapital erwirbt nun die EG-BGes eine Beteiligung an dem entsprechenden AKP-Unternehmen beziehungsweise beteiligt sich an dem zu gründenden Joint Venture. Die anfallenden Dividenden werden zwischen der EG-BGes und dem EG-Investor geteilt, wobei die Ausschüttung - falls eine entsprechende Vereinbarung besteht - auch in Form von Bergbauerzeugnissen geleistet werden könnte.

Nach dem Ablauf einer vereinbarten Frist (z.B. 10 Jahre) hat der EG-Investor folgende Optionen:

- Verlängerung der Laufzeit um eine weitere Periode;

- Einlösen des Schuldscheines bei der EG-BGes und damit Veräußerung der Beteiligung; oder

- Hergabe des Schuldscheines und Übernahme der Beteiligung gegen Zahlung eines Aufgeldes an die EG-BGes.

Folgende Vorteile könnten sich aus diesen europäischen Beteiligungen an AKP-Bergbauunternehmen ergeben:

- Dem beträchtlichen Rückgang der Investitionsbereitschaft von Unternehmen aus EG-Ländern in den AKP-Staaten, der gelegentlich Anlaß zur Besorgnis gibt, ${ }^{22}$ kann entgegengewirkt werden.

- Durch die europäischen Beteiligungen könnte - über ein Erstarken der Lobby - Einfluß auf die Wirtschaftspolitik genommen werden. Wirt-

22 Vgl. Wawrzik, K.: Bericht ..., a.a.O., S. 62; Rajana, C.: Europe undermined, a.a.O., S. 17. 
schaftspolitische Fehler sind, so die herrschende Meinung, die folgenschwersten Ursachen für die prekäre Lage der Entwicklungsländer. ${ }^{23}$

- Die europäischen Gesellschaften haben nicht nur ein kurzfristiges Interesse an den AKP-Unternehmen - während der Lieferung von Ausrüstungsgegenständen oder während des vorübergehenden Bezuges von Bergbauprodukten -, sondern sind durch ihre Beteiligungen an einer generellen Prosperität des AKP-Unternehmens interessiert.

- Die europäischen Unternehmen können ihre Absatz- und Beschaffungsmärkte erweitern beziehungsweise haben die Möglichkeit, zu diversifizieren.

- Die AKP-Bergbauunternehmen erhalten von den Europäern aufgrund der langfristigen Interessen bereitwilliger einen Know-how- und Personaltransfer.

- Der Umfang der Beteiligungen ist nicht an den finanziellen Rahmen der gegenwärtigen Sysmin-Fazilität gebunden.

Da bei dieser "Sysmin-Fazilität mit Beteiligungsoption" die politischen und wirtschaftlichen Risiken durch die EG-BGes, d.h. die EG-Kommission übernommen würden, dürfte die Akzeptanz des Verfahrens bei EGUnternehmen gegeben sein.

Auch in den Augen der AKP-Staaten werden die Vorteile einer solchen Regelung etwaige Nachteile, wie die Bedenken vor einer Unterwanderung des strikten Lomé-Prinzips der Nichteinmischung in innere Angelegenheiten, aufwiegen.

Die stärksten Vorbehalte gegenüber einem solchen Verfahren könnte die EG-Kommission vorbringen, da verstärkt Mittel für dieses Verfahren bereitgestellt werden müssen, wenn sich die EG-Investoren für die Verkaufsoption entscheiden. Um dieses Risiko zu minimieren, es vermindert sich bereits um die Einnahmen aus Dividenden sowie die Aufschlagszahlungen aus den Übernahmeoptionen, müßte:

23 Vgl. O.V.: Larosiere: Es gibt kein Patentrezept für die Schuldenkrise, in: FAZ, 25. September 1984. 
- ein separater Fonds eingerichtet werden, der nicht überzogen werden darf (Volumen wäre zu verhandeln);

- wie bei der Sysmin-Fazilität eine Projektprüfung (Machbarkeit, Wirtschaftlichkeit) erfolgen;

- eine breite Streuung hinsichtlich der AKP-Staaten als auch der mineralischen Rohstoffe angestrebt werden.

Der erfolgreiche Verlauf einiger exemplarischer Beteiligungen könnte rasch weitere Aktionen nach sich ziehen.

Sollte sich trotz des Bemühens der EG-Kommission kein Erfolg bei der Realisierung solcher Beteiligungsprojekte einstellen, könnte in einer weiteren Phase auf eine weniger weitreichende Verbindung zwischen EG- und AKP-Industrie zurückgegriffen werden. Eine Möglichkeit wäre in einem auch in OECD-Bergbauländern praktizierten - Verfahren zu sehen, wobei rohstoffverarbeitende Unternehmen einer Bergbaugesellschaft Kredite einräumen, welche durch Rohstofflieferungen getilgt werden ${ }^{24}$

Obwohl bei dieser Geschäftsbeziehung die Bindungen wesentlich weniger intensiv sind als in der oben erwähnten "Sysmin-Fazilität mit Beteiligungsoption" und die Praxis zeigt, daß Entwicklungsländer Vereinbarungen über Rohstofflieferungen genauer einhalten als finanzielle Absprachen, ${ }^{25}$ wird, um eine rege Anwendung zu erzielen, auch bei einer solchen Regelung - aufgrund der in manchen AKP-Staaten herrschenden politischen Unsicherheit - von der EG-Kommission ein Rahmenabkommen mit Garantieübernahme zu erbringen sein.

\subsection{Ansatzpunkte für weitere Maßnahmen der Europäischen Ge- meinschaft auf dem AKP-Bergbausektor}

Die finanziellen Maßnahmen der Lomé-Abkommen stellen vielseitige und umfassende Instrumente dar. Gleichwohl sind die Mittel nicht ausrei-

${ }^{24} \mathrm{Vgl}$. Wagstyl, S.: Innovations in mine financing, in: Financial Times, 15. August 1986.

${ }^{25}$ Persönliches Gespräch, Metallgesellschaft Service Company, Trade Finance. 
chend, um eine nachhaltige Belebung der AKP-Wirtschaft herbeizufuihren beziehungsweise die grundlegenden Probleme in den Empfängerstaaten $\mathrm{m}$ beheben.

Daher ist es dringend geboten, die Zusammenarbeit zwischen den LoméUnterzeichnerstaaten nicht nur auf finanzielle Aspekte zu konzentrieren. Neben der Notwendigkeit, die Qualität der finanziellen Zusammenarbeit bei jeder Maßnahme zu hinterfragen, ist zu untersuchen, inwieweit eine Intensivierung der technischen Zusammenarbeit die finanziellen Aktionen unterstützen und effektivieren kann.

Allerdings ist auch hierbei die allseitige Akzeptanz solcher "Qualitätssteigerungen" Voraussetzung.

Im folgenden sollen drei Konzepte aufgezeigt werden, bei denen die geforderten Prämissen "Akzeptanz" und "Steigerung der Wirkung der finanziellen Maßnahmen" gegeben sein könnten.

\section{Absatzförderung:}

Zunächst sollte in einer Art "Rohstoffübereinkommen für AKP-Bergbauerzeugnisse" zwischen den Produzenten und Verbrauchern aus den Unterzeichnerstaaten der Lomé-Abkommen versucht werden, enge Kontakte zu knüpfen. Die in Lomé IV zum ersten Male etablierte Regelung zur Einsetzung eines "Grundstoffausschusses" (Lomé IV: Art. 75 f.) ist zu wenig präzise und zu sehr allgemein gehalten, um erfolgreich zu sein.

Ein solches Rohstoffübereinkommen auf nicht-staatlicher Ebene, jedoch unter der Leitung der EG-Kommission oder eines AKP-EG-Gremiums, könnte dem Auf- und Ausbau von Handelsbeziehungen, der Bildung von längerfristigen Liefer- und Abnahmekontrakten und der Absatzförderung dienen. Dies wäre vor allem für kleinere Rohstoffproduzenten hilfreich, wohingegen große und alteingesessene Bergbauunternehmen in der Regel auf altbewährte Handelsbeziehungen zurückgreifen können. ${ }^{26} \mathrm{Zu}$ den Treffen, die im halbjährlichen Turnus stattfinden könnten, sollten Repräsentanten der Bergbauunternehmen, der Rohstoffhändler und der weiterverarbeitenden Industrie geladen werden.

26 Persönliches Gespräch, ZCCM-Headoffice, Lusaka, Sambia. 
Zusätzlich zu diesem AKP-EG-Rohstoffuibereinkommen ließen sich das Angebot und die Nachfrage für AKP-Bergbauprodukte in der AKP-EGRegion durch die Errichtung einer AKP-EG-Rohstoffbörse transparenter gestalten. Diese Institution ist nicht als Konkurrenz zu den bestehenden Rohstoffbörsen a verstehen, sondern soll lediglich Funktionen übernehmen, die von den zur Zeit existenten Institutionen nicht wahrgenommen werden. Neben der Zulassung von kleinen AKP-Anbietern ist es vordringlich, auch bisher nicht börsennotierte Rohstoffe zu erfassen.

Eine Grundvoraussetzung ist, daß an der AKP-EG-Rohstoffbörse verschiedene Bergbauerzeugnisse in festgelegten Qualitätsgraden angeboten und gehandelt werden. Mengenmäßige Beschränkungen sollten nicht existieren, um auch kleineren Anbietern beziehungsweise Abnehmern den Handel zu ermöglichen.

Wöchentlich zu veröffentlichende Notierungen von sofortigen und terminierten Angebots- und Abnahmeofferten garantieren einen aktuellen Kenntnisstand über den AKP-EG-Markt für Bergbauerzeugnisse. Dadurch wird - zusätzlich zu den Transaktionen - eine hohe Markttransparenz erreicht, die den AKP-Bergbaugesellschaften wertvolle Entscheidungsgrundlagen liefert, auch beim Aushandeln von längerfristigen Lieferkontrakten oder bei Geschäften, die nicht über die Börse zustande kommen. Von dieser Transparenz werden besonders AKP-Unternehmen profitieren, die weit von dem europäischen Markt entfernt sind; beispielsweise dann, wenn entschieden werden muß, ob der Liefervertrag mit einem europäischen oder einem "benachbarten" Abnehmer (Süd-Süd-Handel, USA, Japan) abgeschlossen werden soll. Angesichts der erhöhten Markttransparenz könnte sich durchaus eine Stabilisierung der Rohstoffpreise einstellen, vorausgesetzt es gelingt durch geeignete Maßnahmen zu verhindern, daß die börsennotierten Rohstoffe der Spekulation ausgesetzt sind.

Da eine solche AKP-EG-Rohstoffbörse erhebliche Etablierungskosten verursacht und eine beachtliche Vorlaufzeit benötigt, wäre für die Übergangszeit ein vereinfachtes Verfahren zu empfehlen:

Ähnlich dem Vorgehen des Zentrums für Industrielle Entwicklung (CDI), welches in dem zweimonatlich erscheinenden AKP-EG-Organ, The Courier, Kontakte zwischen EG- und AKP-Unternehmen vermittelt, könnten in diesem Journal Verkauf- und Kaufangebote für mineralische Rohstoffe vorzugsweise für nichtbörsennotierte - publiziert werden. Anhand dieser vereinfachten Vorgehensweise wäre es möglich, vor der Gründung einer 
eigenen Börse zunächst die Akzeptanz der jeweiligen Marktteilnehmer zu testen.

Know-how-Transfer:

Ausschließlich finanzielle Maßnahmen bieten keine Gewähr für einen erfolgreichen Stabilisierungsprozeß oder sogar einen ausgewogenen Entwicklungsprozeß. Daher ist es unumgänglich, den AKP-Staaten beziehungsweise den dort ansässigen Unternehmen auch die zu ihrer Entwicklung notwendigen Kenntnisse zukommen zu lassen. Dies gilt besonders für den technologieintensiven Bergbausektor. Während durch finanzielle Aktionen häufig nur die Gelegenheit geschaffen wird, Investitionsgüter, Bergbauausrüstungen und Ersatzteile in Industrieländern zu erwerben (s. Absch. 4.3), kann durch Know-how-Transfer der Weg zur größeren wirtschaftlichen Selbständigkeit geebnet werden.

Die in Lomé IV erstmalig vorgesehenen Möglichkeiten zur Zusammenarbeit bei der Verbesserung des Ausbildungsstandes der Arbeitskräfte im Bergbau (Lomé IV: Art. 99 f.) ist der richtige erste Schritt. Darüber hinaus könnte die Errichtung einer zentralen Anlaufstelle für Know-howTransfer - wobei wiederum das CDI als Beispiel heranzuziehen wäre - der Kenntnisübertragung förderlich sein. Ähnlich wie bei der oben beschriebenen AKP-EG-Rohstoffbörse wird den AKP-Bergbau- und EGIndustrieunternehmen durch diese Kontaktstelle eine größere Transparenz von Bedarf und Angebot ermöglicht. Im Gegensatz zu der in Lomé IV vorgesehenen "Bedarfsanforderung" durch den AKP-Staat (Lomé IV: Art. 100) vereinfacht ein ständig zur Verfügung stehendes Büro das Verfahrensprozedere.

Die Akzeptanz dieser Institution wird erhöht, wenn durch Rahmenvereinbarungen Positionen wie Investitions- und Patentschutz, Lizenzvergabe, Zahlungs- und Finanzierungsmodalitäten verbindlich geregelt würden. Durch eine Einbindung der EG-Kommission werden die AKPEngagements der europäischen Industrie eine zusätzliche Risikominderung erfahren, da eine etwaige Vertragsverletzung gegenüber einer supranationalen Organisation folgenschwerer sein könnte als gegenüber einem einzelnen Unternehmen.

Weiterhin wäre durch Regelungen Sorge zu tragen, daß etwaige finanzielle Mittel der EG-Kommission beziehungsweise Europäischen Investi- 
tionsbank tatsāchlich nur für den Know-how-Transfer im Bergbausektor verwendet werden.

Personalaustausch:

Als eine spezielle Art des Know-how-Transfers kann die Entsendung von Beratern und Fachkräften angesehen werden, die über die Vereinbarungen der technischen Zusammenarbeit (Lomé IV: Art. 275 ff.) hinausgehen.

Nicht selten leiden die AKP-Staaten unter einem Mangel an qualifizierten Arbeitskräften. Im Rahmen der Entkolonialisierung wurden vielfach ausländische Fachkräfte aus den Entwicklungsländern abgezogen, nicht weiterbeschäftigt oder vertrieben - Einheimische sollten die entstandenen Lücken auffuillen.

Es stellte sich jedoch heraus, daß die ausländischen Spezialisten nicht in dem Maße und dem Tempo ersetzt werden konnten, wie es den Wünschen der Verantwortlichen beziehungsweise der Notwendigkeit entsprach.

Zur Behebung des Personalmangels - besonders in den mittleren Führungsebenen - reicht der eigene Nachwuchs nicht aus, die Rekrutierungskampagnen im Ausland scheitern häufig an den hohen Personalkosten für die ausländischen Experten. So erklärt sich, daß beispielsweise in den einzelnen Abbaubetrieben der Zambia Consolidated Copper Mines (ZCCM) durchschnittlich 10 bis 15 Prozent der Stellen in den mittleren Führungsebenen (Geologen, Ingenieure etc.) nicht besetzt sind. ${ }^{27}$

Kraft der Errichtung einer AKP-EG-Vermittlungsstelle für qualifizierte europäische Fachkräfte könnte dieser Mangel gemildert werden.

Durch eine Übernahme oder zumindest eine Kofinanzierung der Personalkosten durch die EG-Kommission - über das Maß der bisherigen Berater hinaus - und die Vereinbarung von Arbeits-(zeit-)Verträgen nach internationalen Maßstäben wird eine generelle Zustimmung zum Personaltransfer garantiert. Die vermittelten Fachkräfte erhalten sowohl akzeptable Gehälter in stabilen Währungen als auch die in Europa üblichen Sozialleistungen. Die EG-Länder können durch die Vermittlung ihren teilweise

27 Persönliches Gespräch, Chefgeologe, Nkana-Division, ZCCM, Kitwe, Sambia. 
angespannten Arbeitsmarkt entlasten. Die AKP-Bergbauindustrie erhält auf diese Weise qualifizierte Arbeitskräfte, die für das einzelne Unternehmen aufgrund der EG-Kostenbeteiligung beziehungsweise -übernahme nicht so kostenintensiv sind wie Experten, die auf dem freien Markt rekrutiert werden müssen.

Auch sollte die Vergabe von Stipendien und Praktika für AKP-Staatsbürger durch diese Vermittlungsstelle erwogen werden. Zwar werden über die Sysmin-Fazilität zum Teil bereits mehrwöchige Betriebspraktika in europäischen Unternehmen - aufgrund der Sprachbarrieren hauptsächlich im Vereinigten Königreich oder in Frankreich - finanziert, dennoch erscheint es sinnvoll, wenn die Dauer der Schulungskurse verlängert und die Anzahl der Plätze erhöht würde. ${ }^{28}$

Durch Erfahrungen in den EG-Industrien kann neben einem Wissenstransfer auch die Wettbewerbsfähigkeit europäischer Erzeugnisse gegenüber dem Weltmarkt gestärkt werden. Da die Stipendiaten während ihrer Zeit in den EG-Unternehmen mit den jeweiligen Anlagen und Maschinen beziehungsweise Produkten vertraut werden, ist es wahrscheinlich, daß sie auch nach ihrer Rückkehr in das AKP-Unternehmen - z.B. als Verantwortlicher für den Zentraleinkauf - auf diese - ihnen bekannte Erzeugnisse zurückgreifen werden.

Aufgrund der allgemeinen Vorteile, die sich durch die Vermittlung von Fachkräften und Stipendiaten sowohl für die AKP-Staaten als auch für die EG-Mitgliedsländer ergeben könnten, erscheint die Akzeptanz einer solchen Einrichtung gewährleistet zu sein. Auch das Aufbringen der finanziellen Mittel über die Sysmin-Fazilität hinaus dürfte sich als nicht allzu schwierig erweisen, da diese bei anderen Programmen (z.B. nationale Arbeitslosenunterstützung in den EG-Staaten, Mittel für Ausbildungsmaßnahmen in AKP-Staaten) zum Teil eingespart oder umgewidmet werden könnten.

Durch Regelungen muß jedoch sichergestellt werden, daß die AKP-Stipendiaten nach Beendigung ihrer Ausbildung wieder in ihre Heimatländer zurückkehren und dort in Positionen eingesetzt werden, die ihren Kenntnissen entsprechen. Darüber hinaus sollte gewährleistet sein, daß die fortgebildeten Arbeitskräfte auch weiterhin den Bergbauunternehmen erhalten bleiben. Wenn das Personal, nachdem es sich gewisse Fähigkeiten erwor-

Persönliches Gespräch, EG-Delegation, Lusaka, Sambia. 
ben hat, kündigt, um z.B. in der nächsten Stadt eine eigene $\mathrm{Kfz}$-Werkstatt zu betreiben, ${ }^{29}$ ist dies zwar nicht zum Nachteil für die Volkswirtschaft des jeweiligen AKP-Staates, dem betroffenen Bergbauunternehmen jedoch ist damit nicht geholfen. In solchen Fällen hätten die begrenzten (Sysmin)Mittel sinnvoller eingesetzt werden können.

\subsection{Ansatzpunkte für eine Erweiterung des Sysmin-Verfahrens über das Lomé-Abkommen hinaus}

In der jüngeren Diskussion über die Sysmin-Regelung wird auch darüber debattiert, inwieweit ein solches Verfahren für Nicht-AKP-Staaten etabliert beziehungsweise die Sysmin-Regelung ausgedehnt werden sollte.

Bereits während der UNCTAD-Konferenz 1985 in Genf signalisierte die Europäische Gemeinschaft die Bereitschaft, ein Verfahren in Anlehnung an die Stabex-Regelung für Nicht-AKP-Staaten etablieren zu wollen. Dabei wurden auch Industriestaaten, die nicht der Europäischen Gemeinschaft angehören, eingeladen, sich an dem System zur Kompensation von Exporterlösrückgängen zu beteiligen.

Im Februar 1987 ist dann ohne Partizipation anderer Industrieländer von der Europäischen Gemeinschaft ein neues Stabex-System für acht LCD's in Lateinamerika und Asien eingerichtet worden ( $\mathrm{s}$. Kap. 2.2 ). Dabei ist im wesentlichen die Lomé-Stabex-Regelung übernommen worden. Aufgrund der Abhängigkeit Bangladeschs wurde in dem Stabex-Warenkatalog zusätzlich Jute erfasst. Ein Transfergesuch wird anders als bei dem LoméStabex-Verfahren nur berücksichtigt, wenn die betroffenen Erzeugnisse nach der Europäischen Gemeinschaft exportiert werden. Die Mittel werden erst nach der Genehmigung durch die EG-Kommission transferiert und sind zweckgebunden in dem beeinträchtigten Sektor oder im Rahmen von Diversifikationsvorhaben einzusetzen.

Der Fonds für das Stabex-System für lateinamerikanische und asiatische Staaten wurde mit 50 Mio. ECU für 5 Jahre ausgestattet. Sollten die jährlich zur Verfügung stehenden Mittel von $10 \mathrm{Mio}$. ECU nicht ausreichen, beobachtet werden; Persönliches Gespräch, Beratender Ingenieur der Metallgesellschaft, Frankfurt/M. 
um die genehmigten Anträge zu befriedigen, werden die Auszahlungen pro rata gekürzt. ${ }^{30}$

Anhand der Etablierung des neuen Stabex-Verfahrens läßt sich insbesondere die Bereitschaft der EG-Kommission und auch der EG-Mitgliedstaaten erkennen, sowohl eine Entwicklungszusammenarbeit mit LDC außerhalb der AKP-Gruppe einzugehen als auch bewährte Verfahren aus dem Lome-Abkommen zu übernehmen. Daß dabei an den Verfahren Modifikationen vorgenommen werden, die teilweise zu restriktiveren Konditionen führen als bei den Regelungen in den Lome-Abkommen ist akzeptabel und im Hinblick auf die geringere Erfahrung bei der Zusammenarbeit mit diesen Entwicklungsländern auch zu begrüßen.

Angesichts der Bereitschaft der Europäischen Gemeinschaft zur Etablierung des neuen Stabex-Verfahrens sollte prinzipiell nichts gegen die Schaffung eines Sysmin-Verfahrens für Bergbaustaaten, die nicht der AKP-Gruppe angehören, sprechen. Es lassen sich sogar hinreichend Gründe anführen, die eine solche Vorgehensweise sogar als zwingend notwendig erscheinen lassen. Dazu gehört zunächst die mehrfach erwähnte Tatsache, daß die Europäische Gemeinschaft als größter Importeur von Bergbauerzeugnissen (s. Abschn. 3) bereits enge Kontakte zu Bergbauländern, die nicht zur AKP-Gruppe gehören, unterhält. Aus diesen Staaten werden zum Teil wichtige Rohstoffe oder Bergbauerzeugnisse bezogen, die von den AKP-Bergbauländern nicht exportiert werden. Eine gewisse Unterstützung zur Aufrechterhaltung der Produktions- und Ausfuhrkapazitäten in diesen Bergbauländern kommt somit auch deutlich den Interessen der Europäischen Gemeinschaft entgegen. Es muß sogar davon ausgegangen werden, daß die Europäische Gemeinschaft an einer derartigen Fazilität ein größeres Interesse hat als an der Stabex-Regelung für NichtAKP-Staaten. Im entwicklungspolitischen Bereich gibt es neben den auch für die Sysmin-Regelung in Lomé gültigen Argumenten vor allem umweltrelevante Aspekte, die das Etablieren einer modifizierten Sysmin-Regelung fordern. Bekanntlich führt der Bergbau und die Weiterverarbeitung der Rohstoffe, wie zum Beispiel die Verhüttung, bei nicht sachkundigem Betreiben der Anlagen beziehungsweise bei veralteten Anlagen zu erheblichen Umweltschäden, die oftmals ganze Regionen beeinträchtigen. Im Hinblick auf Maßnahmen, wie sie auch auf dem Umweltgipfel im Juni

30 Vgl.: Kerbert, M.: The Stabex system for Latin America and Asian developing countries, in: The courier, Nr. 117, September-Oktober 1989, S. $94 \mathrm{f}$. 
1992 in Rio de Janeiro gefordert wurden, erscheint die regionale Ausdehoung eines bereits eingeführten und bewährten Verfahrens für die Bergbau- und Grundstoffindustrie, welches auch im Umweltbereich eingesetzt werden kann, sehr sinnvoll zu sein.

In diesem Zusammenhang ist jedoch darauf hinzuweisen, daß, wie im Falle der Stabex-Regelung, ebenfalls Modifikationen gegenüber der Sysmin-Regelung in Lomé erforderlich sind. So sollten im Hinblick auf die Rohstoffvorkommen in Lateinamerika und Asien nicht nur die im SysminKatalog erfassten Bergbauerzeugnisse berücksichtigt werden. Weiterhin sollte in einer Ergänzung zur Auslöseschwelle sichergestellt sein, daß die Mittel diejenigen Bergbauländer erhalten, die sie am nötigsten brauchen und nicht diejenigen, welche die größten Schwankungen bei ihren Exporteriösen aufweisen.

Gelegentlich wird auch diskutiert, ob eine solche Fazilität für Bergbauerzeugnisse nicht gemeinsam von der Europäischen Gemeinschaft und dem WF initiiert werden solle. Obgleich prinzipiell nichts gegen eine gemeinsame Etablierung eines solchen Verfahrens spricht, wird ein solches Konzept an dieser Stelle aus folgenden Gründen abgelehnt. Erstens müssen die etwaigen Vorteile eines gemeinsamen Systems als nur sehr gering angesehen werden. Ein höheres Mittelaufkommen gegenüber einem parallelen Vorgehen, wo beispielsweise beide Institutionen eigene Fazilitäten bereitstellen, kann nicht erwartet werden. Zweitens erscheint es unwahrscheinlich, daß sich beide Initiatoren zügig auf eine Regelung einigen werden. Während die Europäische Gemeinschaft bereits auf ein bewährtes System für Bergbauerzeugnisse zurückgreifen kann, kann der IWF dem nichts vergleichbar Konkretes entgegensetzen, auch nicht mit seiner Fazilität zur Kompensierung von Exportausfällen und unerwarteten externen Störungen (CCFF). ${ }^{31}$ Langwierige Verhandlungen über die zu vereinbarenden Ziele des Systems sowie verwässerte Kompromiss-Regelungen wären die Folge. Drittens ist anzuzweifeln, ob die gemeinsame Fazilität, die von zwei großen Institutionen gesteuert wird, effektiv arbeiten kann. Die Sorge, daß die Bürokratie noch aufwendiger wird als sie bereits bei den jeweiligen Verfahren der Institutionen selbst ist, erscheint nicht unbegründet.

31 Vgl. Internationaler Währungsfonds (Hrsg.): Jahresbericht des Exekutivdirektoriums für das am 30. April 1991 abgelaufene Geschäftsjahr, Washington, D.C. (1992), S. 66 f.; UNCTAD (Hrsg.): Compensatory financing for export earnings shortfalls, a.a.O. 
Daher wird an dieser Stelle der Appell an die EG-Kommission gerichtet, mit der Etablierung eines modifizierten Sysmin-Verfahrens für NichtAKP-Staaten zu beginnen. Sollten andere Staaten oder supranationale Institutionen Bereitschaft zeigen, sich an dem System beteiligen zu wollen, kann die Europäische Gemeinschaft die Konditionen, zu denen das geschehen kann, festlegen. Sollte es dazu nicht kommen, stehen international eine Vielzahl von Fonds für eine Kofinanzierung zur Verfügung. 


\section{ZUSAMMENFASSUNG}

Im Dezember 1989 ist das Vierte AKP-EWG-Abkommen von Lomé unterzeichnet worden und nach seiner Ratifizierung im Jahre 1991 in Kraft getreten. Mit diesem Abkommen wurde die bereits seit Mitte der siebziger Jahre bestehende, traditionell enge Zusammenarbeit zwischen der Europäischen Gemeinschaft und den Staaten des afrikanischen, karibischen und pazifischen Raumes (AKP-Staaten) vertieft und auf eine breitere Basis gestellt. Die Qualität dieser Vereinbarungen muß sich in den nächsten Jahren sowohl aufgrund der Etablierung des gemeinsamen Europäischen Marktes als auch angesichts der durch die rasche Öffnung Osteuropas entstandenen Aufgaben einer erneuten Bewährungsprobe unterziehen.

Bereits seit der ersten UN-Konferenz für Handel und Entwicklung (UNCTAD) 1964 in Genf ist die Suche nach neuen Formen der Zusammenarbeit zwischen Industrie- und Entwicklungsländern auf dem rohstoffwirtschaftlichen und rohstoffpolitischen Bereich intensiviert worden.

Einer der Schwerpunkte im Ersten AKP-EWG-Abkommen von Lomé wurde auf die Erlösstabilisierung von Rohstoffexporten (Stabex-Verfahren) gelegt. Darin fand die starke Abhängigkeit der AKP-Staaten von Rohstoffausfuhren ihren Niederschlag.

Während in der Stabex-Regelung des Lomé-I-Abkommens neben agrarischen Rohstoffen auch noch mineralische Rohstoffe (Eisenerz und Schwefelkiesabbrände) erfaßt wurden, ist für die Bergbauerzeugnisse in den nachfolgenden Abkommen eine eigene Regelung (Sysmin) entwickelt und ausgebaut worden.

Die in der Sysmin-Regelung von Lomé II noch recht deutlich formulierten rohstoffpolitischen Interessen der Europäer treten in den nachfolgenden Versionen zugunsten der entwickungspolitischen Aspekte in den Hintergrund.

Die Zielsetzung dieser Arbeit war es, die Rolle der Sysmin-Regelung in den Lomé-Abkommen zu untersuchen und die Praktikabilität und Wirksamkeit der Fazilität für Bergbauerzeugnisse aufzuzeigen sowie Alternativen herauszuarbeiten. 
Nach einer Darstellung der wesentlichen entwicklungspolitischen Theorien wurde zunächst detailliert auf die Entstehung und den Inhalt der LoméAbkommen sowie deren Fortentwicklung bis zum Lome-IV-Abkommen eingegangen. Ein Schwerpunkt lag in der Schilderung der Überlegungen, die zur Einführung der Sysmin-Regelung geführt haben, wobei auch die Erweiterung sowohl der Anspruchsgrundlagen als auch der Mittelverwendung Berücksichtigung fand. Dabei ist auch der Unterschied zwischen der Sysmin-Fazilität und dem Stabex-System herausgearbeitet worden. Dieser Sachverhalt wird vielfach bis heute nicht richtig wiedergegeben.

Nachfolgend wurde die Situation auf den Märkten mineralischer Rohstoffe in den achtziger Jahren dargestellt. Damit verbunden war die Darlegung der Problematik, die sich aus der gegenwärtigen Lage sowohl für die AKP-Bergbauländer als auch für die Europäische Gemeinschaft ergibt.

Schließlich wurden Erwartungen und Ansprüche an das Sysmin-Verfahren aufgeführt. Eine kritische Gegenüberstellung mit der bisherigen Praxis, ermöglicht durch zwei, in den Jahren 1984 und 1989 durchgeführte, empirische Erhebungen in Sambia, legte dar, wo es zu einer Diskrepanz zwischen Theorie und Praxis kommt. Dabei ließ das Aufzeigen von Alternativen die Schwachstellen des Sysmin-Verfahrens (auch gemäß Lomé IV) sichtbar werden.

Gleichzeitig wurde aber auch die Schwierigkeit deutlich, Vereinbarungen zu treffen, die - aufgrund der gegensätzlichen Auffassungen der Verhandlungspartner und der begrenzten EG-Mittel - den divergierenden Ansprüchen der Lomé-Unterzeichner Genüge leisten können. In dieser Diskussion ist ein Teil der Ausführungen den sehr komplexen Texten der Sysmin-Artikel in Lome IV gewidmet, deren Auslegung zu Differenzen zwischen Antragsteller und EG-Kommission führen könnte.

Als Resultat der Untersuchung in Sambia läßt sich festhalten, daß sich die Sysmin-Regelung in den Augen der betroffenen Bergbaugesellschaften bewährt hat. Da die Sysmin-Mittel direkt den in Schwierigkeiten geratenen Unternehmen zugute kommen und nicht - wie in der Stabex-Regelung - den AKP-Regierungen zur freien Verfügung überlassen werden, bieten sie den betroffenen Abbaugesellschaften eine willkommene Unterstützung. So konnten bei der Zambia Consolidated Copper Mines Ltd. mit Hilfe von Mitteln aus der Sysmin-Fazilität neben der Anschaffung dringend benötigter Bergbauausrüstungen Rehabilitationsprogramme finanziert werden, so daß es unter Mitwirkung anderer Kreditgeber gelang, zumindest 
für einige Zeit einen weiteren Verfall der für Sambia lebenswichtigen Produktionskapazitäten zu verhindern.

Wie die Erhebungen in Sambia weiterhin ergeben haben, werden die Ausschreibungsmodalitäten der Sysmin-Regelung im Vergleich zu anderen Verfahren - beispielsweise zu dem System der Weltbank (IBRD) - als unkompliziert und praktikabel angesehen.

Ein entscheidender Nachteil liegt für die betroffenen Unternehmen in der langen Zeitspanne zwischen der Antragstellung und der Genehmigung durch die EG-Kommission. Auch verschiedene Maßnahmen zur Beschleunigung dieses Genehmigungsprozederes haben bisher keine wesentliche Abhilfe schaffen können. Ebenso ungünstig wirkt sich für die Bergbaugesellschaft die lange Ungewißheit über die Darlehenshöhe aus.

Trotz der Mōglichkeit, die Sysmin-Mittel auch zur Diversifikation einzusetzen, berücksichtigt die Sysmin-Regelung übergeordnete entwicklungspolitische Belange nur unwesentlich. Insbesondere Maßnahmen zur Strukturverbesserung, die gerade in den vom Bergbau abhängigen AKPStaten erforderlich sind, lassen sich durch die Sysmin-Regelung nur dann finanzieren, wenn bereits laufende Entwicklungsprogramme durch einen Exporterlösrückgang der entsprechenden Bergbauerzeugnisse gefährdet werden. So sind auch in der Sysmin-Regelung von Lomé IV keine konkreten Maßnahmen konzipiert worden, um vor- oder nachgelagerte Industrien zu fördern und erst recht nicht, um solche zu schaffen.

Unter diesen Aspekten ist es auch fraglich, ob die langen Laufzeiten der Darlehen (40 Jahre) an die Bergbaugesellschaften bei den gegenwärtigen Rückzahlungsmodalitäten überhaupt sinnvoll sind. Bei den extraktiven Industrien besteht die berechtigte Sorge, daß in zwei oder drei Jahrzehnten durch die erfolgte Ausbeutung der Lagerstätten nicht mehr genügend Mittel aufgebracht werden können, um die Schuldendienstzahlungen an den AKP-Staat $z u$ leisten.

Aufgrund der hohen und einschränkenden Abhängigkeits- und Auslöseschwellen kann nur eine kleine, ohnehin schon durch ein hohes Geopotential bevorzugte Gruppe von AKP-Staaten die Mittel der Sysmin-Fazilität in Anspruch nehmen.

Auch der Aspekt einer europäischen Rohstoffsicherung darf bei der Sysmin-Regelung nicht überbewertet werden. Die Bergbau-Fazilität ist nicht 
in der Lage, eventuelle Engpässe bei der Versorgung der Europäischen Gemeinschaft mit mineralischen Rohstoffen zu vermeiden. Obwohl von der europäischen Industrie einige Bergbauprodukte in zum Teil beträchtlichen Mengen aus AKP-Staaten bezogen werden (z. B. Kobalt), besteht mittelfristig nahezu immer die Möglichkeit, auf andere Bezugsquellen auszuweichen. Kurzfristig auftretende Versorgungsschwierigkeiten jedoch lassen sich auch nicht mit Hilfe der Sysmin-Mittel der Lomé-Abkommen beheben.

Einen Anreiz für die AKP-Staaten, ihre Bergbauerzeugnisse verstärkt nach der Europäischen Gemeinschaft zu liefern, um somit dem dortigen Interesse nach Bezugsdiversifizierung genüge zu leisten, bietet das SysminVerfahren nicht. In diesem Zusammenhang ist auch darauf hinzuweisen, daß eine Vielzahl der für die Europäische Gemeinschaft strategischen Rohstoffe in der Sysmin-Regelung der Lomé-Abkommen überhaupt nicht explizit erfaßt ist.

Dennoch kann festgehalten werden, daß das Sysmin-Verfahren in Ansätzen durchaus positiv zu beurteilen ist. Dies trifft besonders seit Lomé III zu, wo speziell vom entwicklungspolitischen Standpunkt aus beachtliche Verbesserungen vorgenommen wurden. Mit Hilfe der Sysmin-Mittel können seither auch Diversifizierungs- und Anschlußprojekte finanziert werden. Weiterhin ist die Regelung seit Lomé III bezüglich der Abhängigkeitsschwellen ausgedehnt worden. Zusätzlich zu den Konditionen von Lomé II - mindestens 15 Prozent der Exporterlöse werden durch Ausfuhren eines Sysmin-Rohstoffes ${ }^{1}$ erzielt - können seither auch AKPStaaten von dem Sysmin-Verfahren profitieren, die mindestens 20 Prozent ihrer Erlöse aus dem Export ihrer gesamten mineralischen Rohstoffe ${ }^{2}$ beziehen. Seit Lome IV werden die Mittel der Sysmin-Fazilität an die AKPStaaten als Zuschüsse vergeben, welche den Bergbaugesellschaften dann nach wie vor als langfristige Darlehen zur Verfügung gestellt werden.

Trotz dieser bisher vorgenommenen Verbesserungen ließe sich die Bergbauregelung durch Modifikationen weiter optimieren.

1 Bei den Sysmin-Rohstoffen handelt es sich um Kupfer und Kobalt, Phosphat, Mangan, Bauxit und Aluminiumoxid, Zinn, Eisenerz und Schwefelkiesabbrände, Gold und Uran.

2 Ausgeschlossen sind Erdöl und -gas, Edelmetalle und -steine. 
So sollten für die begünstigten Bergbaugesellschaften neben den bisherigen Rückzahlungsmodalitäten ${ }^{3}$ der Sysmin-Darlehen weitere Tilgungsverfahren konzipiert werden. Damit würde zum einen die Problematik umgangen werden, daß die extraktiven Industrien unter Umständen vor Ende der Darlehenslaufzeit nicht mehr existieren und die Darlehen nicht mehr zurückgezahlt werden könnten. Zum anderen würde so die Möglichkeit geschaffen auch nach internationalen Maßstäben als kreditwürdiges Unternehmen auftreten zu können.

Akzeptable Lösungen stellen flexible Schuldendienstzahlungen oder auch Kompensationsgeschäfte in Form von Rohstofflieferungen nach der Europäischen Gemeinschaft dar. Die letztgenannte Möglichkeit käme den Versorgungsinteressen der Gemeinschaft entgegen, da aufgrund dieser Regelung der Anreiz geschaffen würde, mineralische Rohstoffe bevorzugt in der Europäischen Gemeinschaft abzusetzen.

Zusätzlich sollte der Versuch unternommen werden, verstärkt europäisches Kapital - beispielsweise zum Aufbau von Zulieferunternehmen oder der weiterverarbeitenden Industrie - nach den AKP-Staaten zu leiten. Hierbei müßten in den Lomé-Abkommen die Rahmenbedingungen - u.a. Investitionsschutz, Bürgschaften durch die EG-Kommission - geregelt werden, so daß die allseitige Akzeptanz garantiert wäre.

In der Sysmin-Regelung sollte neben der finanziellen Kooperation auch die technische Zusammenarbeit intensiviert werden. Europäische Berater durch die Sysmin-Fazilität finanziert - könnten über längere Zeiträume in den betroffenen Unternehmen der AKP-Länder Problemlösungen erarbeiten, würden so zu einem Know-how-Transfer beitragen und könnten die sinnvolle Verwendung der Sysmin-Mittel garantieren. Auf diese Weise ließen sich auch die in den AKP-Unternehmen häufig anzutreffenden Engpässe an qualifiziertem Personal mildern - ein Aspekt, dem in Zukunft noch verstärkte Bedeutung zukommen wird, wie die empirischen Untersuchungen im sambischen Kupfergürtel ergeben haben. Die europäischen Berater könnten auch verstärkt darauf einwirken, daß Maßnahmen im Umweltschutz durch die Mittel aus dem Sysmin-Fonds realisiert werden.

Abschließend wird noch auf die Diskussion über die Ausdehnung des Sysmin-Verfahrens auf Nicht-AKP-Staaten eingegangen. Nicht nur im Hinblick auf die EG-Rohstoffversorgung wird eine solche Ausdehnung be- 
fürwortet, sondern auch aufgrund der globalen Umweltdiskussion. Die Sysmin-Mittel können nämlich auch im Umweltbereich eingesetzt werden und dies bei einer Industrie, die in den Entwicklungsländern nach wie vor in erheblichem Maße zur Beeinträchtigung der Umwelt beiträgt. 


\section{SUMMARY}

The realisation of the extent of the increase in the destruction of the global environment and the rapid disintegration of the Eastern bloc has resulted in the emergence of a number of new aspects in development cooperation work over recent years. The tasks now being faced as a result demand both the discovery of new solutions and the adoption of old, tried and tested ones. The European Community has been intensifying its activities in the area of development since the mid 1970s, and the cornerstone of its multilateral work has been the Lome agreements. These agreements between the European Community and 69 developing countries from the African, Caribbean and Pacific areas (ACP nations) have placed fundamental emphasis on stabilising income from raw material exports (Stabex), with the aim of making allowance for the high level of dependence of the ACP states on raw material exports. Since the second Lome agreement (1981 - 1985) a separate process for mining products (Sysmin) has been established after it was revealed that the characteristics of mineral raw materials are substantially different from those involved in the agricultural sector. The Sysmin process has been substantially modified and expanded in the third and fourth Lomé agreements (1986 - 1990; $1990-1999)$.

The aim of this report is to carry out a functional analysis of the Sysmin rule - which has now become very complex - to reveal the level of efficiency and develop proposals for optimisation, which will also be relevant to the new challenges in development work.

To start with, in a summarising discourse, the various theories to explain the underdevelopment as well as the development strategies will be discussed. Then the development policy of the European Community, the creation and the content of the Lomé agreement and its continued development up to the most recent agreement (Lomé IV) will be examined in detail.

Then follows an analysis of the markets for mineral raw materials in the seventies and eighties, whereby particular attention will be paid to the export-economic aspects for the ACP mining countries and the consumerpolitical aspects for the European Community. 
A critical comparison of expectations and demands against practice in the past - made possible by empirical studies in Zambia where the Sysmin rule was first applied - reveals the discrepancy between theory and reality. In the following, listing the possibilities for optimisation highlights the weaknesses of the Sysmin rule, which has already been considerably modified in Lomé IV.

As a result of the empirical studies carried out in mines operated by Zambia Consolidated Copper Mines (ZCCM), it was established that the Sysmin regulation has in principle proven to be successful. Since the favoured ACP states pass the Sysmin loan shares to the companies in difficulties on advantageous terms, this ensures that the problem areas receive direct assistance, unlike the case with other facilities. However, one important disadvantage for the companies concerned is the long period of time between submission of application and approval by the EC Commission.

From the point of view of development policy, the Sysmin regulation is not very effective. Firstly the facility can only be used by a small group of ACP nations already blessed with a high geo-potential. Secondly the facility is not designed to subsidise upstream or downstream industries or to initiate the production increases desired.

Nor should the aspect of securing raw materials for the EC with the Sysmin rule be overestimated. This facility cannot help avoid any consumption bottlenecks, especially since a number of the strategic materials for the European Community cannot be mined in the ACP nations or included in the Sysmin system.

Despite the improved Sysmin rule of Lome IV it would be possible, as the report concludes, to increase the efficiency of the facility by implementing additional measures, for example through agreements concerning alternative terms of repayment or agreements to promote private investment. These measures could then be applied to the new tasks of global development cooperation. 
Anhang 1: Wichtige Eckdaten der AKP-Staaten

Land

Status Bevölkerung BSP/Kopf Exportanteil am Hauptexportprodukte (1 000)

(US-\$)

BSP

(in \%)

Afrika (46)

Äquatorial-Guinea

a

397

330

27

Kakaobohnen, Kaffee

Äthiopien

a $\quad 46144$

120

8 Kaffee, Rinder, Schafe

Angola

9421

$\left.842^{*}\right)$

31

Erdöl, -erzeugnisse

Benin

a

4454

380

27

Baumwolle, Kakaobohnen

Botsuana

$a, b$

1164

2590

59

Fleisch, Diamanten,

Kupfer, Nickel

Burkina Faso

$a, b$

8546

350

11

Rinder, Baumwolle, Erdnüsse

Burundi

a,b

5149

210

10

Kaffee, Edelsteine, Tee

Dschibuti

a

$\left.110 \quad 1050^{*}\right)$

60

Häute, Felle

Elfenbeinküste

$11587 \quad 740^{*}$ )

k.a.

Rohkaffee, Kakaobohnen, Rohhölzer 
Fortsetzung: Anhang 1

\begin{tabular}{|c|c|c|c|c|c|}
\hline Land & Status & $\begin{array}{l}\text { Bevölkerung } \\
\quad(1000)\end{array}$ & $\begin{array}{l}\text { BSP/Kopf } \\
\text { (US-\$) }\end{array}$ & $\begin{array}{c}\text { Exportanteil am } \\
\text { BSP }\end{array}$ & $\begin{array}{l}\text { Hauptexportprodukte } \\
\text { (in \%) }\end{array}$ \\
\hline Gabun & & 1077 & 3780 & 50 & $\begin{array}{l}\text { Erdölprodukte, Mangan- } \\
\text { erze, Rohhölzer }\end{array}$ \\
\hline Gambia & a & 822 & 360 & 63 & Ölsaaten, Erdnüsse \\
\hline Ghana & & 14040 & 400 & 17 & Kakaobohnen, Aluminiumoxid \\
\hline Guinea-Conakry & $\mathbf{a}$ & 6641 & 450 & 27 & $\begin{array}{l}\text { Bauxit, Aluminiumoxid, } \\
\underline{\text { Aluminium }}\end{array}$ \\
\hline Guinea-Bissau & $\mathbf{a}$ & 940 & 190 & 9 & $\begin{array}{l}\text { Erdnüsse, Ölsamen, } \\
\text {-nüsse, -früchte }\end{array}$ \\
\hline Kamerun & & 11213 & 940 & 22 & Kaffee, Rohhölzer, Kakao \\
\hline Kap Verde & $a, c$ & 352 & 750 & 21 & Fisch, Bananen \\
\hline Kenia & & 23021 & 340 & 27 & Kaffee, Tee, chem. Produkte \\
\hline Komoren & $\mathrm{a}, \mathrm{c}$ & 442 & 500 & 16 & Ylang-Ylang, Vanille, Kopra \\
\hline Kongo & & 2100 & 1120 & 42 & Erdöl, Rohhölzer, Diamanten \\
\hline
\end{tabular}




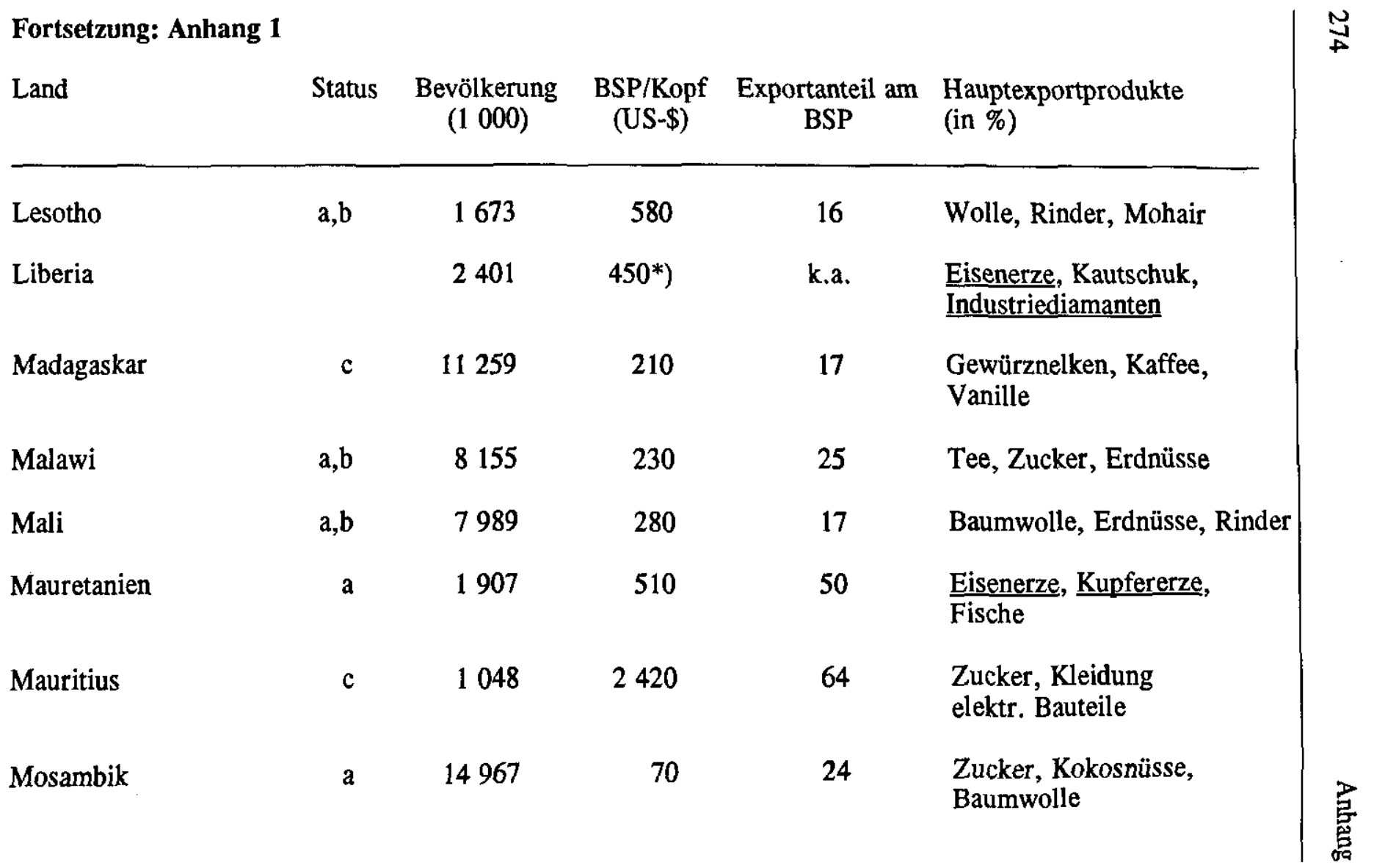


Fortsetzung: Anhang 1

\begin{tabular}{|c|c|c|c|c|c|}
\hline Land & Status & $\begin{array}{l}\text { Bevölkerung } \\
\quad(1000)\end{array}$ & $\begin{array}{l}\text { BSP/Kopf } \\
\text { (US-\$) }\end{array}$ & $\begin{array}{c}\text { Exportanteil am } \\
\text { BSP }\end{array}$ & $\begin{array}{l}\text { Hauptexportprodukte } \\
\text { (in \%) }\end{array}$ \\
\hline Namibia & & 1184 & 1120 & 55 & $\begin{array}{l}\text { Diamanten, Uranoxid, } \\
\text { Fische }\end{array}$ \\
\hline Niger & $a, b$ & 6998 & 300 & 16 & $\begin{array}{l}\text { Uranerze, Rinder, } \\
\text { Hülsenfrüchte }\end{array}$ \\
\hline Nigeria & & 110131 & 290 & 38 & $\begin{array}{l}\text { Erdöl, Kakaobohnen, } \\
\text { Kokosnüsse }\end{array}$ \\
\hline Ruanda & $a, b$ & 6657 & 260 & 12 & Kaffee, Tee, Zinn \\
\hline Sambia & $\mathrm{b}$ & 7486 & $420 \#)$ & 32 & Kupfer, Kobalt, Zink \\
\hline $\begin{array}{l}\text { Sao Tomé und } \\
\text { Principé }\end{array}$ & $a, b$ & 119 & 350 & 25 & Kakaobohnen, Kopra \\
\hline Senegal & & 7154 & 720 & 26 & $\begin{array}{l}\text { Erdnußöl, Phosphate, } \\
\text { Ölkuchen }\end{array}$ \\
\hline Seychellen & $a, b$ & 68 & 5110 & 74 & Kopra, Zimt \\
\hline
\end{tabular}


Land

Status Bevölkerung BSP/Kopf Exportanteil am

Hauptexportprodukte $(1000) \quad$ (US-\$) $\quad$ BSP $\quad$ (in \%)

\begin{tabular}{lrrrrl}
\hline $\begin{array}{l}\text { Sierra Leone } \\
\text { Simbabwe }\end{array}$ & a & 3938 & 210 & 19 & $\begin{array}{l}\text { Diamanten, Eisenerze, } \\
\text { Kaffee }\end{array}$ \\
$\begin{array}{lcccc}\text { Somalia } \\
\text { budan }\end{array}$ & 9257 & 620 & k.a. & $\begin{array}{l}\text { Tabak, Ferrochrom, } \\
\text { Baumwolle, Kupfer, Nickel }\end{array}$ \\
a & 5882 & $\left.170^{*}\right)$ & 10 & Schafe, Bananen, Rinder \\
$\begin{array}{l}\text { Swasiland } \\
\text { a }\end{array}$ & 23776 & $400 \#)$ & 7 & $\begin{array}{l}\text { Baumwolle, Erdnüsse, } \\
\text { Ölsaaten, -nüsse }\end{array}$ \\
$\begin{array}{l}\text { Tansania } \\
\text { a,b }\end{array}$ & 737 & 1060 & 78 & $\begin{array}{l}\text { Zucker, Hölzer, } \\
\text { Weißasbest }\end{array}$ \\
Togo & a & 24739 & 100 & 18 & $\begin{array}{l}\text { Kaffee, Baumwolle, } \\
\text { Gewürznelken }\end{array}$ \\
Tschad & a & 3362 & 410 & 42 & $\begin{array}{l}\text { Phosphate, Kakaobohnen } \\
\text { Kaffee }\end{array}$ \\
& a,b & 5399 & 220 & 19 & $\begin{array}{l}\text { Baumwolle, Rindfleisch, } \\
\text { Rinder }\end{array}$
\end{tabular}


Fortsetzung: Anhang 1

Land

Status Bevölkerung BSP/Kopf Exportanteil am

Hauptexportprodukte

惌 (1 000)

(US-\$) BSP (in \%)

Uganda

$\mathrm{a}, \mathrm{b}$

16195

160

9

Rohkaffee, Baumwolle, Tee

Zaire

33615

220\#)

25

Kupfer, Zink, Erdöl, Diamanten

Zentralafrika-

$\mathrm{a}, \mathrm{b}$

2794

390

15

Kaffee, Diamanten,

nische Republik

$\underline{\text { Karibik (15) }}$

Antigua und

Barbuda

$a, b$

$84 \quad 4770$

k.a.

K.A. (Zucker)

Bahamas

c

$247 \quad 11720$

k.a.

Erdölprodukte, chem. Produkte, Getränke, Tabak

Barbados

c

$255 \quad 6630$

63

Zucker, Kleidung, elektr. Bauteile

Belize

a

$182 \quad 2050$

56

Zucker, Maschinen, Fahrzeuge 
Fortsetzung: Anhang 1

Land

Status Bevölkerung

$(1000)$

BSP/Kopf Exportanteil am

(US-\$)

BSP

Hauptexportprodukte (in \%)

Dominika

a,c

81

2440

54

Dominikanische

Republik

c

6859

950

$102 \quad 2180$

799

290

6254

370

Haiti

c

6254

2429

1380

Jamaika

c

St. Christopher und Nevis a,c

43

3960
12

72

27

58

78

$\frac{\text { Bauxit, }}{\text { Zucker }}$

$\frac{\text { Bauxit, Aluminiumoxid, }}{\text { Zucker }}$

Maschinenbauerzeugnisse, Nahrungsmittel, Bearbei-

tete Waren

Bauxit und Aluminiumoxid, Alkohol. Getränke, Bananen

Zucker, Textilien, Elektrokleingeräte

Bananen, Grapefruit,

Zucker, Ferrolegierungen, Kaffee

Kakaobohnen, Bananen

47 
Fortsetzung: Anhang 1

Land

Status

Bevölkerung (1 000)

BSP/Kopf

Exportanteil am

Hauptexportprodukte BSP (in \%)

St. Lucia

a,c

145

69

Bananen, Kokosnüsse

St. Vincent und

a,c

122

1730

k.a.

Bananen, agrar. Produkte

die Grenadinen

Surinam

429

3610

27

Aluminiumoxid, Bauxit, Rohaluminium, Reis

Trinidad und

c

1241

3620

44

Erdölprodukte, Zucker

Tobago

Pazifik (8)

Fidschi
Kiribati
Papua-Neuguinea

c

732

1830

63

Zucker, Fisch

a,c

67

750

k.a. Phosphate, Kopra

c $\quad 3804 \quad 820$

39

Salomonen

$a, c$

304

560

54

Kupfererze, Kaffee, Kakaobohnen 
Fortsetzung: Anhang 1

\begin{tabular}{lccccc} 
Land & Status & $\begin{array}{c}\text { Bevölkerung } \\
(1000)\end{array}$ & $\begin{array}{c}\text { BSP/Kopf } \\
\text { (US-\$) }\end{array}$ & $\begin{array}{c}\text { Exportanteil am } \\
\text { BSP }\end{array}$ & $\begin{array}{l}\text { Hauptexportprodukte } \\
\text { (in \%) }\end{array}$ \\
\hline Tonga & a,c & 101 & 1100 & k.a. & $\begin{array}{l}\text { Kopra, Kokosnüsse, } \\
\text { Bananen }\end{array}$ \\
$\begin{array}{l}\text { Tuvalu } \\
\text { Vanatu }\end{array}$ & a,c & 8 & $680 \#$ ) & k.a. & Phosphate, Kopra \\
West-Samoa & a,c & 151 & 1120 & 37 & Kopra, Fisch, Kakaobohnen \\
\hline
\end{tabular}

Anm.: a) Am wenigsten entwickelte Länder (LLDC) (gemäß Lomé IV: Art. 330)

b) AKP-Binnenstaat (gemäß Lome IV: Art. 333)

c) AKP-Inselstaat (gemäß Lomé IV: Art. 336)

Angaben gerundet;

Angaben für 1991;

\#) Angaben fuir 1990;

\#\#) Angaben für 1988;

*) Angaben geschätzt.

Quelle: Eigene Zusammenstellung nach: Bundesministerium für Wirtschaftliche Zusammenarbeit (Hrsg): Lomé IV; Entwicklungspolitik, Materialien Nr. 82, Bonn (1991), S.129; Weltbank (Hrsg.):

The World Bank Atlas, Washington, D.C., December 1992 
Anhang 2: Die Bedeutung des Bergbausektors für einige AKP-Staaten

\begin{tabular}{|c|c|c|}
\hline & $\begin{array}{l}\text { Anteil des Bergbaues } \\
\text { an der Gesamtausfuhr } \\
\text { (in \%) }\end{array}$ & $\begin{array}{l}\text { Hauptausfuhr- } \\
\text { produkte }\end{array}$ \\
\hline
\end{tabular}

\begin{tabular}{|c|c|c|}
\hline Sambia & 1989: 46 & $\begin{array}{l}\text { Kupfer } \\
\text { Kobalt } \\
\text { Blei, Zink }\end{array}$ \\
\hline
\end{tabular}

Zaire 1987: $54 \quad \begin{aligned} & \begin{array}{l}\text { Kupfer } \\ \text { sonst. Berg- } \\ \text { bauprodukte } \\ \text { Kaffee }\end{array} \\ & \text { M }\end{aligned}$

\begin{tabular}{lll}
\hline Mauretanien $1987: 36$ & $\begin{array}{l}\text { Eisenerze u. } \\
\text {-konzentrate } \\
\text { Fischereiprod. }\end{array}$
\end{tabular}

\begin{tabular}{lll}
\hline Simbabwe $1987: 32$ & $\begin{array}{l}\text { Gold, Nickel, } \\
\text { Asbest } \\
\text { bearbeitete } \\
\text { Waren }\end{array}$
\end{tabular}

Guayana

1989: 33

Bauxit u. Aluminiumoxid 43

Zucker

Reis 
Fortsetzung: Anhang 2

Staat Anteil des Bergbaues Hauptausfuhr- Anteil der EGan der Gesamtausfuhr (in \%) produkte Exporte am Gesamtexport (in \%)

Surinam

1984: 57

Aluminiumoxid

Bauxit

Rohaluminium

Reis

Kiribati

1980: 79

Kalziumphos-

phate

13

Kopra

Anm.: $\quad\left(^{*}\right)$ unsichere Angabe

Quelle: Eigene Zusammenstellung nach: Statistisches Bundesamt Wiesbaden (Hrsg.): Statistik des Auslandes, Länderkurzbericht Sambia 1991, Zaire 1990, Mauretanien 1991, Simbabwe 1990, Guayana 1993, Surinam 1987, Pazifische Staaten 1986, Stuttgart 1986 - 1993. 
Anhang 3: Die Verwendung der Sysmin-Rohstoffe in den EG-Industrien

Handelsformen

Kupfer: $\quad$ Kathoden, Drahtbarren

Blöcke

Blöcke

Recycling $60 \%$

Kobalt: $\quad$ Kathoden, Oxid

Pulver

Recycling $11 \%$

Phosphate: Phosphate

Nebenprodukte aus der Eisen- und Stahlproduktion

$7 \%$ werden nicht über über Bergbaugewinnung erzeugt

Weiterverarbeitung Endverbraucher

\section{Kupfer-Halbzeuge \\ Messing- \\ Gußerzeugnisse}

\section{Legierungen \\ Dauermagnetwerkstoffe \\ Hochfeste Stähle \\ Verbindungen}

$34 \%$ Elektroindustrie

$22 \%$ Bausektor

$17 \%$ Maschinenbau

$9 \%$ Verkehr

$18 \%$ Sonstige

$19 \%$ Eisen- + Stahlindustrie

$18 \%$ Elektroindustrie

$14 \%$ Luft- + Raumfahrtindustrie

$9 \%$ Werkzeugindustrie

$40 \%$ Sonstige

$76 \%$ Landwirtschaft

$11 \%$ Chem. Industrie

$13 \%$ Sonstige 
Handelsformen Weiterverarbeitung Endverbraucher

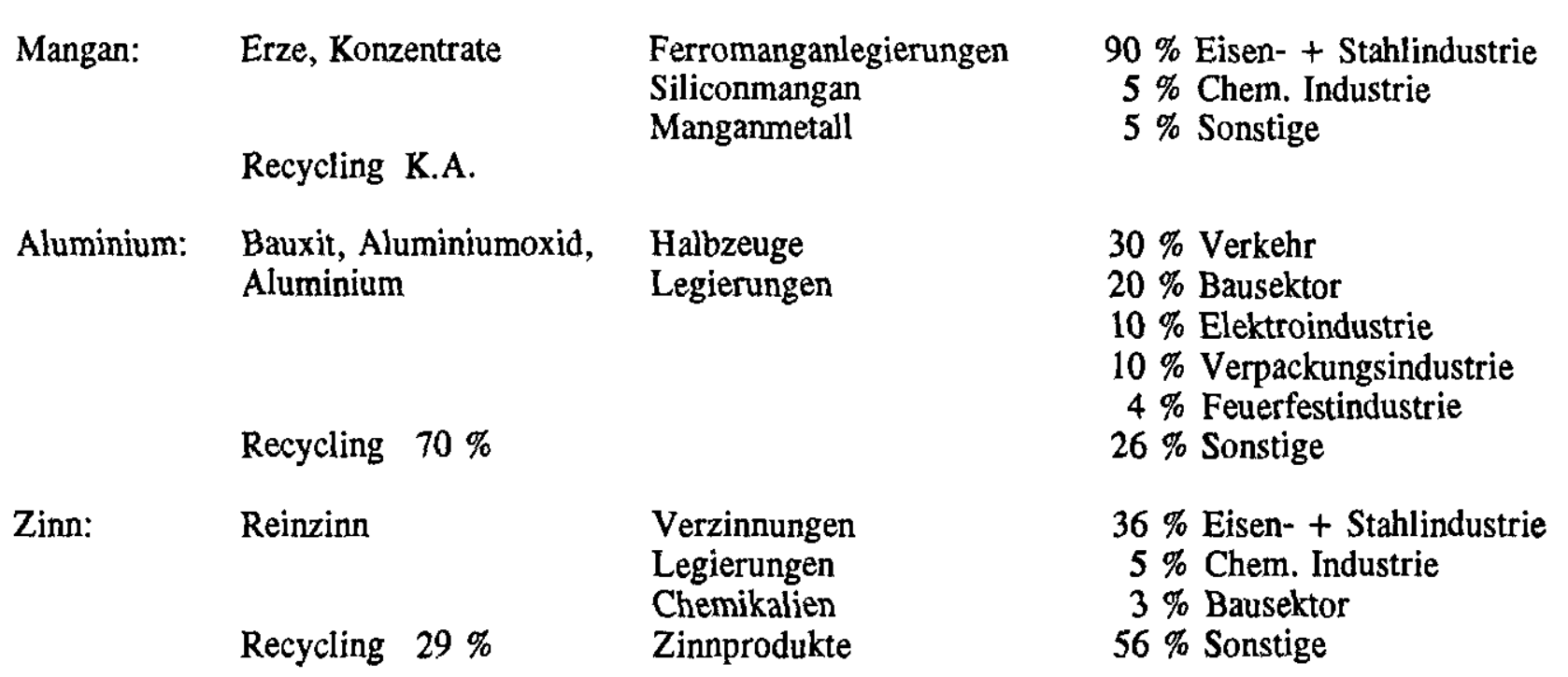


Handelsformen Weiterverarbeitung Endverbraucher

Eisen: $\quad$ Erze, Konzentrate Pellets, Schwefel-

kiesabbrände

Recycling $47 \%$
$90 \%$ Stahl, -Legierungen

$10 \%$ Gießereieisen
$16 \%$ Bausektor

$15 \%$ Transportwesen

$10 \%$ Maschinenbau

$3 \%$ Elektroindustrie

$3 \%$ Verpackungsindustrie

$53 \%$ Sonstige

Anm.: a) Phosphate 1980; Mangan und Kobalt durchschnittlich 1979 - 1981;

Die Recyclingangaben beziehen sich auf das Jahr 1982 (Kupfer 1989, Aluminium 1990).

Quelle: Eigene Zusammenstellung nach: O.V.: Die Aluminium-Industrie rechnet mit einem hohen Materialrücklauf, in: Blick durch die Wirtschaft, 15. April 1991; Gabrisch, R.; Häussler, H.-R.: Wachsende Bedeutung der Sekundärstoffe, in: Handelsblatt, 28. November 1990; Gocht, W. (Hrsg.): Handbuch der Metallmärkte, Berlin 1985; Statistisches Amt der EG (Hrsg.): EG-Rohstoffbilanzen 1979 - 1982, Luxemburg 1985, S. $231 \mathrm{ff}$. 



\title{
Anhang 4: Die Sysmin-Regelung des Zweiten, Dritten und Vierten AKP-EWG-Abkommens von Lomé
}

\author{
TITEL III \\ BERGBAUER?.EUGNISSE \\ Kapitel 1 \\ Hilfe rür Vorhaben und Programme
}

ARTIKEL 49

Um zur scharfung einer stabileren Grundlage für die Entwicklung der AKP-Staaten beizutragen, deren wirtschart in hohem Masse vom gergbau abhängt, und um insbesondere diesen Staaten $z u$ helfen, der Verringerung ihrer Kapazität zur Ausfuhr von Bergbauerzeugnissen nach der Gemeinschaft und der entsprechenden Verringerung inrer Ausfuhrerlöse entgegenzuwirken, wird ein System eingerührt, das diese staaten bei ihren Bemünungen um die Behebung der nachteiligen Auswirkungen unterstützen soll, die die vorübergehenden und von dem Willen der betrofrenen AKPStaaten unabhängigen schweren stbrungen im Bereich des Bergbaus aur inre Einnahmen haben.

\section{ARTIKEL, 50}

(1) Das in Artikel 49 vorgesehene system findet auf folgende Bergoauerzeugnisse Anwendung:

- Kuprer, einschliessich der damit verbundenen kobaltproduktion;

- Phosphate;

- Mangan; 


\section{Fortsetzung: Anhang 4}

- Bauxit und Aluminiumoxyd;

- 2inn;

- Schwefelkiesabbrände und Eisenerze, auch agglomeriert (einschliesslich Pellets), ausgenommen die in Artiked 25 Absatz 2 genannten Fälle während des in diesem Artikel genannten Zeitraums.

(2) Treten für eine oder mehrere Waren, die in dieser Liste nicht erfasst sind, von denen die Volkswirtschaft eines oder mehrerer AKP-Staaten jedoch weitgehend abhangig ist, frühestens 12 Monate nach Inkraftreten dieses Abkommens schwerwiegende störungen auf, so 5ussert sich der Ministerrat spätestens sechs Monate, nachdem die betreffenden AKP-Staaten einen entsprechenden Antrag gestellt haben, zur Aufnahme dieser Wase oder Waren in die Liste.

ARTIKEL 51

(1) Für die in Artikel 49 genannten Zwecke wird für die Lavizeit dieses Abkommens eine besondere Finanzierungsrazidität ceschaffen, für die die Cemeinschalt zur Erfïlurg ihrer gesamten Verpflichtungen im Rahmen dieses Systems eink: Gesantbetrag von 280 Millionen ERE bereitsteilt:

a) Diescr lietrag wird von der Kommission verwaltet. 


\section{Fortsetzung: Anhang 4}

b) Dieser Cesamtbetrag wird entsprechend der Zahl der Anwendunesjahre in gleiche jährliche Tranchen aurgeteilt. Jedes Jahr, ausser in letzten Jahr, kann der Ministerrat auf der Grundlage eines inm von der Kommission vorgelegten Berichts, sofern erforderlich, einen Vorgriff von höchstens 50 \% auf die Tranche des folgenden Jahres genehmigen.

c) Restbeträge am Ende eines jeden Anwendungsjahres dieses Abkommens - mit Ausnahme des letzten Jahres - werden automatisch auf das folgende Jahr übertragen.

d) Reichen die Mittel für ein Antendungsjahr nfcht aus, so werden die rälligen Betrăge entsprechend gekürzt.

e) Die für jedes Anwendungsjahr verfügbaren Mittel bestehen aus cer summe

- der jăhrichen Tranche, gekürzt um die gegebenenfalls aufgrund von Nummer 2 verwendeten Beträge;

- der gemass Nummer 3 ibertragenen Mittel.

(2) Der Ministerrat beschliesst vor Ablauf des in Artikel 188 vorgesehenen Zeitraums über die Verwendung etwaiger Restbestände des Gesamtbetrags. 


\title{
Fortsetzung: Anhang 4
}

\author{
ARTIKEL 52
}

(1)

Die Finanzmittel der besonderen Fazilität nach Artikel 51 können von den gemáss Artikel 53 in Betracht kommenden Ländern in Anspruch genommen werden, wenn festgestelit wird oder in den rolgenden Monaten damit gerechnet werden kann, dass inre Produktions- oder Ausfuhrkapazitäten oder inre Ausfuhrerlöse für eine von Artikel 50 erfasste und nach der Gemeinschaft ausgefihrte ware so stark zurückgehen, dass durch die ernste Gefährdung der Rentabilität einer im übrigen lebensfähigen und wirtschaftlichen Produktion die Entwicklungspolitik des betreffenden AKP-States ernstich in Prage gestellt ist und es dieser dadurch unmöglich wird, dif Produktionsanlagen oder die Ausfuhrkapazität normal zu erneuern oder zu erhaiten.

(2) Die vorstehend genannte Möglichkeit zur Inanspruchnahme des Systems ist ebenfalls gegeben, wenn infolge ernster technischer zwlschencälle oder störungen oder infolge schwerwiegender interner oder externer politischer Ereignisse ein wesenticher Rückgang der Produktions- oder Ausfuhrkapazitäten eintritt oder vorherzusehen ist.

(3) Als wesentlichen Rückgang der Produktions- und Ausfuhrkapazitäten gilt eln Absinken um $10 \%$

\section{ARTIKEL 53}

Ein AKP-Staat, der während der vier vorangegangenen Jahre in der Regel mindestens 15 * seiner Einnahmen aus der Ausfuhr einer von Artikel 50 erfassten Ware bezogen hat, kann eine Finanzhilfe aus den Mitteln der besonderen Finanzierungsfazilitat beantragen, wenn die Bedingungen nach artikel 52 erfüllt sird. 


\section{Fortsetzung: Anhang 4}

Im Falle der in Artikel 155 ADsatz 3 genannten staaten beträgt der im vorstehenden Absatz vorgesehene Satz $10 \%$.

(3) Der Antrag auf Finanzhilfe wird an die Komission gerichtet, die inn in Verbindung mit dem betreffenden AKPStat prür. Die Erfüllung der Bedingungen wird von der Komission und dem AKP-Staat im gegenseitigen Einvernehmen restgestellt. Die von der Komission dem AKP-Stat notifizierte Feststellung gibt letzterem einen Anspruch auf die Finanzhilfe der Gemeinschaft aus den Mitteln der besonderen Pinancierungsfarilität.

\section{ARTIKEL 54}

(1)

Die in Artikel 53 vorgesenene Pinanzhilfe wird zur Errelchung der in Artikel 49 festgelegten $2 i e l e$ verwendet.

12) Der Betrag der Pinanzhilfe zur Pinanzierung von Vorhaben oder Programen wird von der Kommission nach Massgabe der für die besondere Finanzierungsfazilităt verfügbaren Mittel, der Art der von dem betreffenden AKP-Stat vorgeschlagenen Vorhaben oder Programre und der Möglicnkeiten einer Mitfinanzlerung festgesetzt. Bei der Festsetzung dieses Betrags werden der Umfang des Ruckgangs der Produktionsund Ausfuhrkapazitäten und der von den AkP-Staten erlittenen Einnahmeverluste im Sinne von Artikel 52 berücksichtist. 


\section{Fortsetzung: Anhang 4}

(3) In keinem Pall können einem einzigen AKP-staat mehr als 50 \& der im Rahmen der jährlichen Tranche verfügbaren Mittel gewährt werden.

(4) Die Verfahren filn die Gewăhrung einer Hilfe unter den obengenannten Bedingungen und die Durchführungsmodalităten dazu sind in Titel VII festgelegt; sie tragen der Notwendigkeit einer raschen Abwicklung der Hilfe Rechnung. 


\title{
Fortsetxung: Anhang 4
}

Bergoaverzejgnisse: Besondere F1nanzierungstaz\$11tat (SYSMIN)

\begin{abstract}
ARTIKEL 176
Um zur Schaffung einer stabljeren Grundlage fur die Entwicklung der AKP-Staaten belzutragen, deren wirtschaft vom Bergbau abhungt, und un insbesondere diesen staaten zu helren, der Verringerung inrer kapazität zur hussuhr von Eergbauerzeugnissen nach der Geneinschaft und der entsprechenden Verringerung ihrer Ausfuhrerlóse entgegenzuwirken, wird ein System efngefihrt, das diese staaten bel ihren Bemuhungen um die Sanlerung des Bergbausektors bzw. um die Behebung der nachte111gen Auswirkungen unterstüzen sol1, die die voribergehenden oder unvorhersehbaren und von dem willen der betroffenen AKP-Staaten unabhangigen schweren Störungen in Bereich des Eergbaus aut inre Entwicklung haben.
\end{abstract}

ARTIKEL 177

(1) Das in Artikel 176 vorzesehene System findet insbesondere auf folgende Bergbauerzeugrisse Anerering:

- Kupfer, elnschliessilch der camit verbundenen Kobaltfroduktion:

- Phosphate:

- Mangan;

- Bauxit und Aluminfumoxyd:

- 2inn: 


\section{Fortsetzung: Anhang 4}

- Eisenerz (Erze, Konzentrate, Schwefelkiesaborände), auch agglomerlert (einschliesslich Pellets).

(2) Treten fur elne oder mehrere Waren, die in dieser liste niche erfasst sind, von denen die Volkswirtschact eines oder mehrerer AKP-Staten fedoch weltgehend abhangig ist, fruhestens 12 Monate nach Inkraftreten dieses Abkomens schwerwiegende storungen aur, so aussert sleh der Ministerrat spatestens sechs Monate, nachdem die betreffenden AKP-Staaten einen en:sprechencen Antrag gestellt haben, zur Aufnahme dieser Ware oder Waren in die Liste.

ARTIKEL 178

(1) Fur die in Artlkel 176 genannten Zwecke wird fur die Laufzelt dieses Abkommens eine besondere Flnanzlerungsfazilitat geschaffen, fur die die Gemelnschaft zur Erfuliung ihrer gesamten Verpflichtungen im Rahmen dieses Systems einen Gesamtbetrag von 415 M1lionen ECU bereltstellt:

a) pleser Gesamtbetrag wird von der Kommission vemaltet.

b) Er wird entsprechend der 2 ahl der Anwendungsjahre in glelche Jahrliche Tranchen aufgetelit. Jedes Jahr, ausser im letzten Jahr, kann der Ministerrat auf der Grundlage eines inm von der Kommission vorgelegten Berlchts, sofern erforderlich. elnen vorgriff von nöchstens 50 \% aur die Tranche des folgenden Jahres genehmigen.

c) Restbeträge am Ende elnes jeden Anwendungsjahres dieses Abkommens - mit Ausnahme des letzten Jahres - werden automatisch auf das rolgende Jahr übertragen.

d) Relchen die Mittel für ein Anwendungsjahr nicht aus, so werden dle fälligen Beträge entsprechend gekürzt. 


\section{Fortsetzung: Anhang 4}

e) Die fur jedes Anwendungsjahr verfugbaren Mittel bestehen aus der summe

- der Jăhlichen Tranche, gekurzt un die gegebenenfalls Busgrund von Buchstabe b verwendeten Beträge;

- der gemäss Buchstabe c ubertragenen Mittel.

(2) Der Ministerrat beschliesst vor Ablauf des in Artikel 291 vorgesehenen zeltraums uber die verwendung etwalger Restmittel aus dem im vorliegenden Artikel genannten Gesamtbetras.

ARTIKEL 179

(1) Die Mittel der besonderen Fazliltut nach Artikel 178 konnen

a) von den gemäss Artikel 180 Buchstabe a in Betracht kommenden Landern fur elne von Artikel 177 erfasste und nach der Gemeinschaft ausgertinrte ware,

b) von den gemáss Artikel 180 Buchstabe b. nicht aber gemass Artikel 180 Buchstabe a in Betrasht kommenden Landern in Fall rü fall zu bestimmenden Abwelchungen von Artikel 177 und Artikel 180 Buchstabe a

in Anspruch genommen werden, wenn festgestelit wird oder in den folgenden Monaten damit gerechnet widen kann. dass ihre produktlons- oder Ausfunrkapaz1taten oder ihre Ausfuhreriose fir in Artikel 177 und Artikel 180 Buchstabe berfasste Bergbauerzeugnisse so stark zurijckgehen, dass die Rentablitit von im 


\section{Fortsetzung: Anhang 4}

Ubrlgen lebensfunlgen und wirschaftlichen Produktionen ernstlich gefahrdet 19t. mit der Folge. dass die Produktionsanlagen oder die Ausfunrkapazitat nicht normal ernevert oder erhalten werden konnen und die Finanzlerung grosser Entwlcklungsvorhaben unterbrochen wird. Cur die der betreffende AXP-Stal die Erlose aus bergbauerzeugnissen vorrangig verwendet hat.

(2) Die Mittel der besonderen Faz111tat kónnen ebenfalls gemass Absatz 1 in Anspruch genommen werden, wann infolge ernster technischer 2 wschenfalle oder Storungen oder 1 infolge schwerwiegender interner oder externer politischer Erelgnisse oder wlchtiger technologlacher und witschartlicher veranderungen. die die Rentabliltat der Produktion beelntrachtigen. ein wesentlicher Ruckgang der Produktions-oder Ausfuhrkapaz1taten eintrite oder vorherzusetien $1 \mathrm{st}$.

(3) Als wesentlichen Ruckgang der Produktions- und Ausfunrkepazititen gijt eín Absinken un $10 \%$.

ARTIKEL 180

EIn AKP-staat, der in der Regel wahrend zumlndest zwel der vier vorangegangenen Jahre

a) mindestens 15 * setner Austuhrerlose aus der Ausfunr elner von Artikel 177 erfassten Ware, oder

b) In Fall fur Fall zu bestimmenden Abwelchungen von Artikel 177 und von Buchstabe andescens $20 \%$ selner Ausunreribse aus ofer Authohr seiner gesamten Bergbaverzeughisse (ausgenommen Edelmetallerze. Erdol und Gas)

bezogen hat, Kann elne Finanzhilfe aus den Mitteln der besonderen Flnanzlerungsfazil1tat beantragen, wenn die Bedingungen bes Artikels 179 errullt sind. 


\section{Fortsetzung: Anhang 4}

Im Falle der am wenlgsten entwlckelten AKP-Staaten, der AKP. Blnnenstaqten und der AKP-Inselstaaten betragt der in Buchstabe a vorgesenene $5 a t z 10 \%$ und der in Buchstabe b vorgesehene satz $12 \%$.

ARTIKEL 181

Der Antrag auf Finanzhil fe wird an die Kommission gerichtet. die inn in Verbindung $m i t$ dem betrefCenden Axp-staat prilft. Erforderlichenfalis kann ein Eligutachten zur fechnischen und finanziellen Dlagnose der betreffenden Produktionskapazitát aus den in Artikel 178 vorgesehenen Mitteln finanziert werden. un Insbescndere elne raschere Prufung des Antrags zu ermöglichen.

Die Erfullung der Bedingungen wird von der Gemeinschart und dem AKP-Staat $1 \mathrm{~m}$ gegenseltigen Einvernehmen restgestellt. D1e von der Kommission dem AKP-Staat notifizlerte Feststellung gibt letzterem einen Anspruch aut die Finarizhlfe der Gemelnschaft aus den Mitteln der besonderen Finanzíerungsfazl1 1 tät.

\section{ARTIKEL 182}

Die in Artikel 180 vorgesehene Finanzhilre wird zur Errelchirip der in Artikel 176 festgelegten 2 lela verwendet.

Sle dient vorrangig zur Flnanzlerung von Reaktivierungs-. Unterhaltungs- und Rationalisierungivorhaben in Erganzung zu den Benühungen des betreffenden AKP-Staates, die gefánrdete Produktions- und Ausfuhrkapazităt wieder aur elnen wirtschaftlich lebensfahigen stand zu bringen, wobel der reibungs- 


\section{Fortsetzung: Anhang 4}

losen Elngliederung in den Gesamtentwlcklungsprozess des Landes besondere Aufmerksamkeit gewidmet wird. Erweist es sich als unmöglich, die Kapazität auf elnen wirtschaftlich lebensfälgen Stard zu oringen, so ermitteln die Partelen die Vorhaben oder Programme, durch die sich die ziele des Systems am besten verwirkl1chen lassen.

Bel Anwendung von Artikel 179 Absatz 1 Buchstabe o und Artikel 180 Buchstabe $b$ werden die Mittel der besondecen finanzlerungsfazllitat vorrang1g zur Unterstutzung der anstrengungen elngesetzt, die der betreffende AKP-Staat unternimmt, um elne Unterbrecnung der Entwlcklungsvorhaben nach Actikel 179 zu verhindern oder um vorhaben zu fördern, die ge1 gnet $\$ 1$ d, die gefahrdeter. Kapazitaten als Ausfuhrerlosquellen zumlndest tellwelse zu ersetzen.

Der Betrag der Finanzhllfe wird von der Kommission nach Massgabe der fur die besondere Finanzierungsfazllitat verrijgbaren Mittel, der Art der von dem betreffenden Axp-stast vorgeschlagenen Vorhaben oder Programme und der Moglichkelt einer Mitrinanzlerung festgesetzt.

Bel der jestsetzung dieses Betrags werden der Umfang des Rijckgangs der Produktlons- und Ausfunckapazltaten und der von den AKP-Staaten erlittenen Einnahmeverluste im Sinne von Artikel 179 sowle die relat 1 ve Bedeutung der betreffenden Bergbaulndustrle fur die Ausfuhrerlose des AKP-Staates berilcksicntigt.

In kelnem fall können einem einzigen AKP-Staat mehr als 35 * der $1 \mathrm{~m}$ Hanmen des jahrlictien Tranche verfugdaren Mitcel gewărt werden. Im Falle eines Beltrags auf der Grundlage von Artikel 179 Absatz. I Bucnstabe o und Artikel 180 Buchstabe b beträgt dieser Satz $15 \%$. 


\section{Fortsetzung: Anhang 4}

ole verfahren fur die Cewanrung elner Hilfe unter den obengenannten Bedingungen und die Durchrihrungsmodal 1 taten dazu sind in Titel III des Dritten Telis dieses Abkommens restgelegt: sie tragen der Notwendigkelt elner raschen Abwicklung der Hile Rechriung.

ARTIKEL 183

(1) Um vorsorgliche Massnahmen zu ermbglichen, durch die sleh Schaden an Produktionsanlagen wărend der Prurung oder Durchrutrung dieser vorhaben oder Programme verhindern lassen. kann die Cemeinschaft elnem AKP-Staat auf Antrag elne Vorauszahlung gewahren. Diese Moglichielt schliesst nicht aus. dass der AKP-staat die Soforthilfen gemáss Artikel 203 in Anspruch nimmt.

(2) Da die Vorauszahlung zur Vorfinanzlerung der Vorhaben oder Programme gewanrt wird, die mit inr vorbereltet werden, werden bel der festsetzung inrer Hohe Bedeutung und Art dieser Vorhaben oder Programme berucksichtigt.

(3) Die Vorauszanlung wird in form von Lieferungen. Diengtlefstungen oder auch als Barzanlung gewänt, wenn letztere fur geelgneter gehalten wird.

(4) Die Vorauszanlung wird in cen Betrag der Hilifen der Cemeinschaft in Form von Vorhaben oder programmen be 1 der unterzelchnung des diesbeziglichen Finanzierungsabkommens einbezogen. 


\title{
Fortsetzung: Anhang 4
}

\begin{abstract}
ARTIKEL 194
Die aus den Mittein der besonderen Finanzierungsrazlitiat gewdhrten Hilfen werden zu den gleichen bedingungen wie dle Sonderdarlehen zuridckgezahlt, wobel die Bestimmungen zugunsten der an wenlgsten entwlckelten AKP-Staaten berucksichtigt werden.
\end{abstract}

Quelle: AKP-EWG-Ministerrat (Hrsg.): Drittes AKP-EWG-Abkommen, Brüssel 1985, S. 168 ff. 


\title{
Fortsetzung: Anhang 4
}

\author{
Kapitel 3 \\ Bergbauerzeugnisse \\ Besondere Finanzierungsfazilität (SYSMIN)
}

\section{Artikel 214}

(1) Es wird eine besondere Finanzierungsfazilität geschaffen, die an die AKP-Staaten gerichtet ist, für deren Wirtschaft der Bergbau eine wichtige Rolle spielt und in denen in diesem Bereich Schwierigkeiten festzustellen bzw. für die nahe Zukuntt vorhersehbar sind.

(2) Die Zielsetzung des Systems besteht darin, einen Beitrag zur Schaffung einer stabileren oder sogar erweiterten Grundlage für die Entwicklung der AKP.Staaten zu leisten, indem diese unterstützt werden bei ihren Bemühungen

- um den Schutz der Erzeugung und Ausfunr von Bergbauerzeugnissen durch Sanierungs- und Vorbeugungsmaßnahmen, mit welchen den schwerwiegenden volkswirtschaftichen Auswirkungen des Verlusts an Lebensfähigkeit entgegengewirkt werden soll, der aut eine Verringerung der Produktionsbzw. Ausfunrkapazität und/oder der Ausfunreriöse im Bergbausektor infolge einschneidender technologischer oder wirtschatlicher Veranderungen oder infolge vorübergehender oder unvorhersehbarer Störungen zunjckzuführen ist, auf die der betreffende AKP-Staat und das auf dem betroffenen Sektor tätige Unternehmen keinen Einfluß haben; dabei wird der Anpassung der Wettbewertssituation der Unternehmen an die veränderten Marktbedingungen besondere Aufmerksamkeit gewidmet:

- oder um die Diversifizierung und Erweiterung der Grundlagen für ihr Wirtschaftswachstum, wobei den AKP-Staaten, die in starkem Maße von den Ausfuhren eines Bergbauerzeugnisses abhängen, insbesondere bei der Durchtührung ihrer bereits eingeleiteten Entwicklungsprojekte und -programme geholfen wird, wenn diese infolge erheblicher fückgänge der hür dieses Bergbauerzeugnis erzielten Ausfuhrerlöse emsitich gefährdet sind. 


\section{Fortsetzung: Anhang 4}

(3) Im Hinblick auf die Erreichung der genannten Ziele wird

- diese Unterstützung den Umstrukturierungserfordemissen der Wirtschaft des betreffenden AKP.Staates angepaß3t;

- bei der Festlegung und Durchführung der Unterstützungsmaßnahme den beiderseitigen interessen der Vertragsfarteien Rechnung getragen.

\section{Artikel 215}

(1) Die in Artikel 214 vorgesehene besondere Finanzierungsfazilität richtet sich an die AKP-Staaten, deren Ausfuhren in die Gemeinschaft gehen und die während zumindest zwei der vier dem Jahs der Antragstellung vorangegangenen Jahre

a) mindestens $15 \%$ inrer Austuhrerlöse aus der Ausfuhr eines der folgenden Erzeugnisse bezogen haben: Kupfer (einschließlich Kobalt), Phosphate, Mangan, Bauxit und Aluminiumoxid, Zinn, Eisenerz, auch agglomeriert, Uran;

b) oder mindestens 20\% ihrer Ausfuhrertöse aus der Ausfuhr sämtlicher Bergbauerzeugnisse (mit Ausnatume von Edelmetallerzen, außer Gold, Erdöl und Erdgas) bezogen haven.

im Falle der am wenigsten entwicketten AKP.Staaten, der AKP. Binnenstaaten und der AKP-inseistaaten beträgt der in Buchstabe a vorgesehene Satz $10 \%$ und der in Buchstabe b vorgesehene Satz $12 \%$.

Bei der Berechnung der unter den Buchstaben a und b genannten Schwellenwerte bleiben die Erlöse aus der Ausfuhr der Bergbauerzeugnisse, die nicht unter das System fallen, unberücksichtigt. 


\section{Fortsetzung: Anhang 4}

(2) Die besondere Finanzienungsfazilität kann in Anspruch genommen werden, wenn im Lichte der vorgenannten Zlele

a) festgestelit oder damit gerechnet wird, daß die Lebensfähigkeit eines oder mehrerer Untemehmen des Bergbausektors durch vorübergehende oder unvorhersehbare materielle, wirtschaftliche oder politische Efeignisse emsthaft beeinträchtigt wurde oder werden wird, auf die der betreffende Staat und das betreffende Unternehmen keinen Einfluß haben, und wenn diese Beeinträchtigung der Lebensfähigkeit in einem merklichen Rückgang der Einkünfte des betreffenden AKP-Staates - wofür im besonderen ein Rückgang der Produktions- oder Austuhrkapazität bei dem betreffenden Erzeugnis um etwa $10 \%$ maßgebend ist - und/oder einer Verschlechterung seiner Leistungsbilanz zum Ausdruck kommt bzw. kommen könnte.

Kennzeichnend für eine voraussictrtliche Beeinträchtigung der Lebensfähigkeit ist es, wenn eine Verschlechterung des Zustands der Produktionsanlagen einsetzt, die Auswirkungen auf die Volkswirtschaft des betreffenden Landes hat;

b) in dem in Absatz 1 Buchstabe a genannten Fall festgestelt wird, daß ein starker Rückgang der Ausfuhrerlöse für das betrettende Bergbauerzeugnis - verglichen mit den Durchschnittseriösen der beiden der Antragstellung vorangegangenen Jahre - die Durchführung bereits eingeleiteter Entwicklungsprojekte oder -programme emstlich gefährdet. Um berücksichtigt zu werden, muß dieser Rückgang

- die Folge materieller, wirtschaftlicher oder politischer Ereignisse sein; er dart woder mittel- noch unmittelbar künstlich durch Politiken und Maßnahmen des betreffenden AKP-Staates oder der betreffenden Wirtschaftsteilnehmer hervorgerufen worden sein;

- zu einem entsprechenden Rückgang von mindestens $10 \%$ der gesamten Ausfuhreriǒse des dem Jahr der Antragstellung vorangegangenen Jahres führen.

Die genannten unvorhersehbaren Ereignisse beziehen sich auf Störungen wie Unfälle, ernste technische Zwischentälle, schwerwiegende interne oder externe politische Ereignisse, wichtige technologische und wirtschaftliche Veränderungen oder erhebliche Veränderungen in den Handelsbeziehungen zur Gemeinschaft. 


\section{Fortsetzung: Anhang 4}

(3) Ein AKP.Staat kann eine Finanzhilfe aus den Mittein der besonderen Finanzierungstazilität beantragen, wenn die Bedingungen đot Absätze 1 und 2 ertüllt sind.

\section{Artikel 216}

(1) Die in Artikel 215 vorgesehene Finanzhilfe wird zur Erreichung der in Artikel 214 Absatz 2 vorgesenenen Ziele verwendet.

- Halten die beiden Parteien die Aufrechterhaltung oder die Wiederherstellung der Lebensfähigkeit eines (der) betroffenen Bergbauuntemehmen(s) für moglich und angebracht, so ist die Finanzhilfe dazu bestimmt. Projekte oder Programme - einschließlich Projekte oder Programme zur finanziellen Umstruk* turierung der betroffenen Untemehmen - zu finanzieren, die die Aufrechtemaltung, Wiederhersteliung oder Rationalisienung der betreffenden Produktions- oder Ausfuhikapazitäten aú einem wirtschattlich lebensfahigen Stand bezwecken.

- Malten die beiden Parteien die Autrechtemaltung oder die Wiederherstellung der Lebensfăhigkeit nicht für möglich, so ist die Finanzhilfe dazu bestimmt, die Grundlagen des Wirtschafts. wachstums durch die Finanzienung von Projekten oder Programmen tür eine lebenstähige Umstellung oder horizontale oder vertikale Diversifiziensng zu enweitem.

- Das Ziel einer Diversifizierung kann im gegenseitigen Einvernehmen auch dann angestrebt werden, wenn der Grad der Abhăngigkeit der Wirtschatt von dem betreffenden Bergbauerzeugnis bedeutend ist, und zwar selbst dann, wenn die Lebensfähigkeit sich wiederterstellen ließe.

- Findet Artikel 215 Absatz 2 Buchstabe b Anwendung, so wird das Ziel einer Diversifizienung im Wege einer Finanzienung angestrebt, die zur Verwirklichung von außerhalb des Bergbas. sektors liegenden Entwicklungsprojekten und -programmen beiträgt, die bereits eingeleitet sind und sonst getahrdet wàren.

(2) In dieser Hinsicht ist bei dem Beschluß über die Zuweisung von Mitteln tür Projekte oder Programmo den wirtschaftlichen interessen und den sozialen Auswirkungen einer solchen Finamzhilfe in dem betreffenden AKP-Staat sowie in der Gemeinschaft gebührend Aechnung zu tragen, wobei der Beschluß den Bedür. nissen der witschattlichen Umstrukturierung dieses AKP.Staates anzupassen ist. 


\section{Fortsetzung: Anhang 4}

Im Rahmen đer Antrāge nach Artikel 215 Absatz 1 Buchstabe b bemühen sich die Gemeinschatt und der betreffende AKP-Staat gemeinsam und systematisch darum, den Anwendungsbereich und die Einzelheiten der etwaigen Finanzhilfe so festzulegen, daß

dem konkurrierenden Bergbau in der Gemeinschaft durch diese Finanzhilfe kein Schaden entstent.

Die Beurteilung und die Berucksichtigung der vorgenannten Aspexte sind Bestandteil der Diagnose nach Artikel 217 Absatz 2.

(3) Besondere Autnerksamkoit gilt

- Cen Tätigkeiten im Bereich der Verarbeitung und des Verkehrs, insbesondere aut regionaler Ebene, sowie der reibungsiosen Eingliederung des Bergbausektors in den Gesamtprozeß der witschaftichen und sozialen Entwicklung des Landes;

- den Vorbeugungsmaßratimen, mit denen sich Störungen im Wege der Anpassung an dis Technologien, der Vervollkomm. nung der technischen und Managementtähigkeiten des ortlichen Personals sowie im Woge der Anpassung der Fähigkeiten des örtichen Personals an die Betriebstührungstechniken der Untemehmen auf ein Mindestmaß beschränken lassen;

- sowie der Starkung des wissenschaftlichen und technologischen Potentials oer AKP-Staaten zur Herstellung neuer Matorialien.

\section{Artikel 217}

(1) Der Antrag auf Finanzhilfe enthălt die Angaben über die Art der aufgetretenen Probleme, die festgesteliten oder erwarteten Auswirkungen der Stönngen sowohl aut Landesebene als auch auf der Ebene des (der) betroffenen Bergbauuntemehmen(s) sowie Angaben (in Form von Merkblāttem) über die Maßnahmen oder Aktionen, die zur Beseitigung der Störung eingeleitet wurden bzw. enwünscht sind.

Der Antrag wird sofort nach Feststellung der vorgenannten Auswirkungen innerhalb einer Frist für die Zusammenstellung des Dossiers yon höchstens zwöll Monaten gestellt. 


\section{Fortsetzung: Anhang 4}

(2) Vor jeder Entscheidung der Gemeinschatt wird systematisch eine technische, wirtschattliche und finanzielle Diagnose des betrotfenen Bergbausektors durchgeführt, um sowohl die Zuläs. sigkeit des Antrags als auch das geplante Projekt oder Nutzungsprogramm zu beurteilen. Bei dieser sehr gründlichen Diagnose werden zur Bestimmung der Finanzhilfe insbesondere die Weltmarktaussichten sowie - unbeschadet des Artikels 216 Absatz 2 Unterabsatz 1 die Lage auf dem Gemeinschaftsmarkt fûr die betreffenden Ereugnisse berücksichtigt. Die Diagnose umiaßt zudem eine Analyse der Auswirkungen, die sich aufgrund einer derartigen Finanzhilfe unter Umstănden tür den konkurrierenden Bergbau in den Mitgliedstaaten ergeben, sowie eine Analyse der Auswirkungen, die sich für den betreftenden AKP.Staat ergeben können, wenn die Finanzhilfe nicht gewährt würde. Anhand dieser Diagnose soll geprüft werden,

- ob die Lebensfảhigkeit der betretfenden Produktionsanlagen beeinträchtigt worden ist oder werden könnte, ob sie wiederhergestellt werden kann oder ob Diversifizierungsmaßnahmen zweckmäßiger erscheinen;

- oder ob der in Artikel 215 Absatz 2 Buchstabe b genannte Rückgang der Austuhrerlöse die Durchführung der bereits eingeleiteten Entwicklungsprojekte oder -programme ernstlich gefährdet.

Diese Diagnose wird nach dem Vertaturen der finanziellen und technischen Zusammenarbeit vorgenommen. Thre Durchführung erfolgt in enger Zusammenarbeit mit dem AKP.Staat und dessen hiertür in Frage kommenden Wirtschaftsteilnehmern.

(3) Förderungswürdigkeit und Finanzierungsvorschlag sind Gegenstand eines einzigen Beschiusses.

Die Gemeinschatt und der AKP-Staat tretfen die ertorderlichen Maßnahmen, um die Prüfung der Anträge voranzutseiben und um eine rasche Durchführung der geeigneten Finanzhilfe zu ermög. lichen. 


\section{Fortsetzung: Anhang 4}

\section{Artixel 218}

(1) Für das Anlaufen und die Überwachung des Projekts kann im Bedartsfall aus den Mittein der Fazilität eine technische Hilfe gewăht wercen.

(2) Die Vertahren für die Gewährung einer solchen Hilfe und die entsprechenden Durchführúngsbestimmungen sind dem die Zusammenarbeit bei der Entwicklungsfinanzienung betreffenden Tell des Abkommens zu entnehmen.

\section{Artikel 219}

(1) Für die in Artikel 214 genannten Zwecke stellt die Gemeinschath tür die Geltungstauer des Finanzprotokolls zu diesem Abkommen zur Extüllung ihrer gesamten Verpflichtungen im Rahmen der besonderen Finanzienungsfazilitat den in diesem Protokoll genannten Gesamtbetrag bereit. Der dieser Fazilität zugewiosene Betrag wird von der Kommission verwaltet.

(2) a) Er wird entsprechend der Zahl der Anwendungsjahre in gleiche jährliche Tranchen aufgeteilt. Jedes Jahr, außer im letzten Jahr, kann der Ministerrat aut der Grundlage eines ihm von der Kommission vorgelegten Berichts, sofem erforderlich, einen Vorgriff von höchstens $50 \%$ auf die Tranche des folgenden Jahres genehmigen.

b) Restbetrăge am Ende sines joden Anwendungsjahres des Finanzprotokolls zu diesem Abkommen - mit Ausnahme des letzten Jahres - werden automatisch aut das folgende Jahr übertragen.

c) Die für jedes Anwendungsjahr verfügbaren Mittel bestehen demnach aus der Summe

- der jảnrlichen Tranche, gekürzt um die gegebenenfalls aufgrund von Buchstsbe a verwendeten Beträge;

- der gemaß Buchstabe b übertragenen Mittel. 


\section{Fortsetzung: Anhang 4}

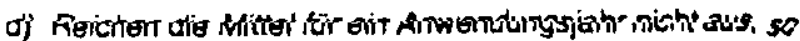
werden unbeschadet der Buchstaben $a, b$ und $c$ die vorgesehenen Beträge entsprechend gekürt.

Der Ministerrat beschließt vor Ablauf der Geltungsdauer des Finanzprotokolis über die Verwendung etwaiger Restmittel aus dem Gesamtbetrag.

(3) Der Betrag der in Artikel 215 vorgesehenen Finanzhilfe wird von der Kommission nach Maß̈gabe der tür die besondere Finan. zierungsfazilität vertügbaren Mittel, der Avt der Durchtührungspro. jekte und -programme, der Möglichkeit einer Mittinanzienthg sowie der relativen Bedeutung der betreffenden Bergbauindustrie für die Volkswirtschaft des AKP-Staates testgelegt.

(4) Auf keinen Fall düren einem einzigen AKP-Staat mehr als $35 \%$ der nach Absatz 2 Buchstabe c vertügbaren Mittel gewähnt werden. Dieser Satz beträgt für die Finanzhilfe nach Artikel 2 is Absatz 1 Buchstabe o $15 \%$.

(5) Die einem AKP-Staat im Rahmen der Finanzierungsfazilität gewährten Hilfen können von diesem an den Enddarlehensneh. mer weitergegeben werden, und zwar zu anderen finanziellen Bedingungen, die im Rahmen des finanzienungsbeschlusses festgelegt werden und sich aus der Prütung des interventions. projekts anhand der üblichen wirtschaftlichen und finanziellen Kriterien für die vorgesehene Projektart ergeben.

(6) Die in Artikel 217 genannte Diagnose wird aus den Mittein der Fazilität finanziert.

(7) In Ausnahmefällen in Zusammenhang mit einer Notsituas, tion, die die Diagnose in einer ersten Phase bestätigen und rechtfertigen mUB, Kann einem AKP-Staat. der einen entsprechenden Antrag stellt, eine Vorauszahlung als teilweise Vortinanzierung cies gepianten Projekts oder Programms gewahnt wercen.

Quelle: Bundesrat (Hrsg.): Entwurf eines Gesetzes zu dem Vierten AKPEWG-Abkommen von Lomé; Bundesratsdrucksache 547/80, Bonn 1990, S. 47 f. 
Anhang 5: Gegenüberstellung der Sysmin-Artikel des Lomé-II- und Lome-III-Abkommens

Lomé II

Lomé III

Änderungen zu Lome II

Art. 49

Art. 176

Art. 50

Art. 177

Art. 51

Art. 178

Art. 52 (Auslöseschwelle)

Art. 179

auch wenn Finanzierungen großer Entwicklungsvorhaben unterbrochen

Art. 53 (Abhängigkeit)

Art. 180

in mindestens zwei der letzten vier Jahre

Art. 53

Art. 180 (a)

Art. 180 (b)

oder $20 \%$ aller Bergbauerzeugnisse (außer Edelmetallen und steinen, Erdöl und -gas); LLDC $12 \%$

Art. 53 Abs. 3

Art. 181

Art. 54 Abs. 1

Art. 182 Abs. 1

und die Regelung der Finanzierung von Studien 


\section{Lomé II}

Art. 54 Abs. 2

$-$

Art. 182 Abs. 3

Art. 54 Abs. 3

Art. 182 Abs, 6

Art. 182 Abs. $2,4,5$

und Alternativen,

Art. 54 Abs. 4

Art. 55

Art. 56

Lomé III

Art. 182 Abs. 7

Art. 183

Art. 184
Änderungen zu Lomé II

falls Wiederherstellung der Kapazitäten nicht mehr möglich

Mittel sollen auch in Anschlußprojekten Verwendung finden, die im Falle eines Rückganges der Bergbaukapazitäten eine Reduzierung der Exporterlöse verhindern

aber nicht mehr als $35 \%$ einer Jahrestranche bzw. $15 \%$ bei Gewährung nach Art. 180 (b)

Quelle: Eigene Zusammenstellung nach: AKP-EWG-Ministerrat (Hrsg.): Zweites AKP-EWG-Abkommen, Brüssel 1981; Derselbe:Drittes AKP-EWG-Abkommen, Brüssel 1985. 
Art. 176

Art. 177

Art. $178 ; 182$ Abs. 4,6

Art. 219

Art. 179 (Auslöseschwelle)

Art. 215 Abs. 2

Art. 180 (Abhäng.-schw.)

Art. 180 (a)

Art. 180 (b)

Art. 181

Art. 182

Art. 214

Art. 215 Abs. 1

Art. 215 (1a)

Art. $215(1 b)$

Art. 217

Art. 216
Art. 215 Abs.1a

s.u.

Festlegung, wie AKP-Staat Mittel an Dritte weitergibt

auch wenn Verschlechterung der Leistungsbilanz; "im übrigen wirtschaftliche Produktionen" nicht mehr Bedingung; Ereignis darf nicht künstlich hervorgerufen worden sein erweitert um Uran

erweitert um Gold

Erfordernis zur Erstellung einer Diagnose

Kein Schaden für EG-Bergbau durch Sysmin 
Fortsetzung: Anhang 5

Lomé III Lomé IV Änderungen zu Lomé III

Art. 218

Finanzierung der Projektüberwachung

Art. 183

Art. 219 Abs. 7

Art. 184

Zuschüsse statt Sonderdarlehen

Quelle: Eigene Zusammenstellung nach: AKP-EWG-Ministerrat (Hrsg.): Drittes AKP-EWG-Abkommen, Brüssel 1985; BMZ (Hrsg.): Lome IV, Bomn (1991). 


\section{LITERATURVERZEICHNIS}

ACP-EEC ASSEMBLEY REPORT ON COMMODITIES (Hrsg.): Commodity studies, in: The Courier, Nr. 116, Juli - August 1989, S. $76-79$.

AKP-EWG-MINISTERRAT (Hrsg.): Zweites AKP-EWG-Abkommen und dazugehörige Dokumente, Brüssel 1981.

AKP-EWG-MINISTERRAT (Hrsg.): Jahresbericht des AKP-EWG-Ministerrates (1982), Brüssel (1983).

AKP-EWG-MINISTERRAT (Hrsg.): Drittes AKP-EWG-Abkommen und dazugehörige Dokumente, Brüssel 1985.

ASANTE, S.K.B.: Lomé II: Alte Abhängigkeit im neuen Gewand?, in: Entwicklung und Zusammenarbeit, Vol. 22, Oktober 1981, S. 7 - 8.

BANK OF ZAMBIA (Hrsg.): Republic of Zambia, Financial Report, Lusaka, Juni 1984.

BANK OF ZAMBIA (Hrsg.): Central statistical office, 0.0 ., Februar 1989.

BERSANI, G.: Dossier: Die Versammlung AKP-EWG 1964 - 1982, Luxemburg, Februar 1983.

BOMSEL, O.: Mining and metallurgy investment in the third world, OECD Development Center Studies, Paris 1990.

BORRMANN, A., et al.: Ausgewählte Modelle und Reformmöglichkeiten der Exporterlösstabilisierung, Forschungsberichte des BMZ, Bd. 56, Köln 1964.

BOUVIER, P.: What to expect from Lome? Neither the best nor the worst!, in: The Courier, Nr. 113, Januar - Februar 1989, S. 10 - 13. 
BRADLEY, K.: Copper Venture, London 1952.

BRAUN, H.-G.: Schulden-Swap und GRIP-Instrumente zor Entschärfung der internationalen Verschuldungskrise?, in: ifo-Schnelldienst 1-2/87, 13. Januar 1987, S. 3 - 5.

BUNDESANSTALT FÜR GEOWISSENSCHAFTEN UND ROHSTOFFE (Hrsg.): Rohstoffwirtschaftliche Länderberichte, XXI Sambia, Hannover, Februar 1979.

BUNDESMINISTERIUM FÜR WIRTSCHAFT (Hrsg.): Mineralische Rohstoffe - Märkte und Perspektiven, Bonn 1982.

BUNDESMINISTERUM FÜR WIRTSCHAFTLICHE ZUSAMMENARBEIT (Hrsg.): Journalistenhandbuch '89/90, Bonn 1989.

BUNDESMINISTERUM FÜR WIRTSCHAFTLICHE ZUSAMMENARBEIT (Hrsg.): Lomé IV; Entwicklungspolitik, Materialien Nr. 82, Bonn (1991)

BUNDESRAT (Hrsg.): Entwurf eines Gesetzes zu dem Zweiten AKPEWG-Abkommen von Lomé vom 31. Oktober 1979 sowie zu den mit diesem Abkommen in Zusammenhang stehenden Abkommen, Bundesratsdrucksache 160/90, Bonn 1980.

BUNDESRAT (Hrsg.): Entwurf eines Gesetzes zu dem Vierten AKPEWG-Abkommen von Lomé vom 15. Dezember 1989 sowie zu den mit diesem Abkommen in Zusammenhang stehenden Abkommen; Bundesratsdrucksache 547/80, Bonn 1990.

BUNDESSTELLE FÜR AUSSENHANDELSINFORMATION (Hrsg.): EWG und AKP-Staaten, Lomé-III-Abkommen, in: Markt-Information, Reihe MI-C, Köln, Februar 1985.

BUNDESSTELLE FÜR AUSSENHANDELSINFORMATION (Hrsg.): Wirtschaftslage, Simbabwe zur Jahresmitte 1988, August 1988.

BUNDESSTELLE FÜR AUSSENHANDELSINFORMATION (Hrsg.): Sambia, September 1988. 
BUNDESSTELLE FÜR AUSSENHANDELSINFORMATION (Hrsg.): Wirtschaftsdaten aktuell, Simbabwe, Oktober 1988.

BUNDESSTELLE FÜR AUSSENHANDELSINFORMATION (Hrsg.): Wirtschaftsentwicklung Sambia, Januar 1989.

BUNDESTAG (Hrsg.): Gesetz zu dem Vierten AKP-EWG-Abkommen von Lomé vom 15. Dezember 1989 sowie zu den mit diesem Abkommen in Zusammenhang stehenden Abkommen; Bundestagsdrucksache 11/8014, Bonn 1991.

CAVALEVU, J.D.V.: Bericht anhand des Jahresberichtes des AKPEWG-Ministerrates fur 1982, und im Hinblick auf die bevorstehenden Verhandlungen über ein Nachfolgeabkommen zu Lomé II, Teil B: Begründung, Beratende Versammlung AKP-EWG, 0.O., 13. September 1983.

CHERRINGTON, J.: The EEC's dairy desperation, in: Financial Times, 18. November 1986.

CHEYSSON, C.: "Our experiment has been converted into a policy, the Lomé-policy", in: The Courier, Nr. 58, November 1979, S. 8 - 9.

CLAUS, B.: Lomé II, Verhandlungspositionen und Ergebnisse, in: GTZ (Hrsg.): Technische Zusammenarbeit in der Entwicklungsplanung, Dokumentation einer Fachtagung vom 24. Nov. bis 5. Dez. 1979, Wölfersheim, S. $158-167$.

COMMENWEALTH FUND FOR TECHNICAL CO-OPERATION (Hrsg.): Papua New Guinea, Informationsmemorandum, o.O., März 1988.

COMMODITIES RESEARCH UNIT (Hrsg.): Copper to 1995, Vol. III, London 1984.

COPLIN, W.; O'LEARY, M.K.: A systematic approach to political risk analysis for extractive industrie, in: CIM Bulletin, 76, Oktober 1983, S. $51-60$. 
DELEGATION DER KOMMISSION DER EUROPÄISCHEN GEMEINSCHAFTEN (Hrsg.): Co-operation Zambia European Communities, Annual Report 1987, Vol. 1 + 2, o.O., Juni 1988.

DENNHARDT, J.; PATER, S. (Hrsg.): Entwicklung muß von unten kommen, Reinbek 1980.

EUROMETAUX (Hrsg.): Lome IV-Sysmin, Comments of the non-ferrous metals industry, unveröffentlichter Entwurf, Brüssel, 21. September 1988.

EUROPÄISCHE INVESTITIONSBANK (Hrsg.): Jahresberichte 1981 1989, Luxemburg 1982 - 1990.

EUROPÄISCHE INVESTITIONSBANK (Hrsg.): 25 Jahre 1958 - 1983, o.O., April 1983.

FOCKE, K.: Von Lomé $1 \mathrm{zu}$ Lomé 2, Texte des Berichts und der am 26.09.80 von der AKP-EWG-Versammlung angenommenen Entschließung, Luxemburg 1980.

FORTIN, C.: Magnitude and impact of the price falls on LCD exporters, in: The Courier, Nr. 116, Juli - August 1989, S. 57 - 59.

FRALON, J., et al.: The new EEC-ACP-convention: from Lome I to Lomé II, Brüssel 1980, zit. in: Rajana, C.: Europe undermined: The Lomé response; An evolution of the EEC-ACP non-fuel-minerals arrangement, in: Africa Development, Vol. VI, Nr. 2, April-Juni 1982, S. 5-36.

FREEMANN, P.V.: Mining in Zambia today, ZCCM (Hrsg.), Lusaka 1983.

FUCHS, G.: Bericht im Namen des paritätischen Ausschusses über die industrielle Zusammenarbeit AKP-EWG und die Auswirkungen des Abkommens von Lomé, Teil B: Begnündung, Beratende Versammlung AKP-EWG Sitzungsdokumente 1983 - 1984, o.O., 24. August 1983. 
FUCHS, G.: Bericht im Namen des paritätischen Ausschusses über die industrielle Zusammenarbeit AKP-EWG und die Auswirkungen des Abkommens von Lomé, Teil: Anhang, Beratende Versammlung AKP-EWG Sitzungsdokumente 1983 - 1984, o.O., 30. August 1983.

GABRISCH, R.; HÄUSSLER, H.-R.: Wachsende Bedeutung der Sekundärstoffe, in: Handelsblatt, 28. November 1990.

GÉCAMINES (Hrsg.): Rapport Annuel 1981, Kinshasa (1982).

GÉCAMINES (Hrsg.): Situation actuelle et perspectives, Lumbumbashi, 13. Februar 1988.

GERDES, U.; BOSSE, P.: Stabex kontra Rohstoff-Fonds, in: epd-DritteWelt-Information 10/75, aktualisierte Fassung 1979.

GERTH-WELLMANN, H.; KAYSER, D.: Die industrielle Zusammenarbeit zwischen der EG und den AKP-Staaten im Rahmen der LoméPolitik, in: Afrika-Spektrum, 15. Jg., 1980/1, S. 37 - 51.

GLASER, T.: Sierra Leone, in: The Courier, Nr. 86, Juli - August 1984, S. $24-28$.

GLASER, T.: EEC-ACP cooperation: the historical perspective, in: The Courier, Nr. 120, März - April 1990, S. 24 - 28.

GOCHT, W.: Wirtschaftsgeologie und Rohstoffpolitik, 2. Aufl., Berlin, Heidelberg, New York, Tokyo 1983.

GOCHT, W. (Hrsg.): Handbuch der Metallmärkte, Berlin, Heidelberg, New York 1985.

GOCHT, W.; ZANTOP, H.; EGGERT, R.G.: International mineral economics, Berlin, Heidelberg, New York, Tokyo 1988.

GRAND, B.: Sysmin, in: The Courier, Nr. 79, Mai - Juni 1983, S. 7 - 8.

GRAND, B.: Sysmin-Mines as instrumentes of international cooperation, in: The Courier, Nr. 94, November - Dezember 1985, S. $82-85$. 
GRIMM, K.: Theorien der Unterentwicklung und Entwicklungsstrategien, Studienbücher der Sozialwissenschaft, Bd. 38, Opladen 1979.

HARMS, U., et al.: Die Investitionspolitik der NE-Bergbaugesellschaften und ihre Auswirkungen auf die Rohstoffversorgung der Bundesrepublik Deutschland, ite, Hamburg 1978.

HARMS, U.; IWERSEN, A.: Rohstoffgewinnung in Entwicklungsländern, ite, Hamburg, Oktober 1981.

HELLDORF, K.v.: Die Abkommen von Lomé: Immer noch Wegweiser?, in: Entwicklung und Zusammenarbeit, Vol. 22, Oktober 1981, S. 9 - 10.

HEMMER, H.R.: Wirtschaftsprobleme der Entwicklungsländer, 2. Aufl., München 1988.

HERDA, M.: Leichtmetalle, in: Gocht, W. (Hrsg.): Handbuch der Metallmärkte, Berlin, Heidelberg, New York 1985, S. 319 - 348.

HERRMANN, R.: On the evaluation of the Stabex-system, in: Intereconomics, Januar - Februar 1982, S. 7 - 11.

HOFFMEYER, M.; SCHRADER, J.V.: Tendenzwandel auf den Rohstoffmärkten, in: Die Weltwirtschaft, Februar 1984, S. 128 - 144.

INSANALLY: Bericht im Namen des Paritätischen Ausschusses über den Fünften Jahresbericht des AKP-EWG-Ministerrates (AKP-EWG 26/81) und eine Untersuchung der ersten Erfahrungen mit dem Zweiten Abkommen von Lomé und Empfehlungen für seine optimale Durchführung, Beratende Versammlung AKP-EWG Sitzungsdokumente 1981 - 1982, 12. Oktober 1981.

INSTITUT FÜR WELTWIRTSCHAFT (KIEL): ACP-EEC Trade (1984), in: The Courier, Nr. 98, Juli - August 1986, S. 61 - 91.

INTERNATIONALER WÄHRUNGSFONDS (Hrsg.): Jahresbericht des Exekutivdirektoriums für das am 30. April 1985 abgelaufene Geschäftsjahr, Washington, D.C. (1985). 
INTERNATIONALER WÄHRUNGSFONDS (Hrsg.): Primary Commodities-Market Developments and Outlock, o.O., Mai 1987.

INTERNATIONALER WÄHRUNGSFONDS (Hrsg.): Jahresbericht des Exekutivdirektoriums für das am 30. April 1991 abgelaufene Geschäftsjahr, Washington, D.C. (1991).

IRMER, U.: Bericht im Namen des Ausschusses für Entwicklung und $\mathrm{Zu}-$ sammenarbeit über das Umfeld des Nachfolgeabkommens von Lomé II, Teil I: Entschließungsantrag, Teil II: Bericht der verschiedenen Unterberichterstatter und Stellungnahmen der mitberatenden Ausschüsse, Europa-Parlament Sitzungsdokumente 1983 - 1984, 12. August 1983.

JÜTTE-RAUHUT, J.: Internationale Marktregulierungen als Risikofaktor bei Projektfinanzierungen im Bergbau, Diss. RWTH Aachen, Internationale Kooperation, Bd. 35, Baden-Baden 1987.

KAISER, M.; WAGNER, N.: Entwicklungspolitik: Grundlagen-Probleme-Aufgaben, Heidelberg 1986.

KERBERT, M.: The Stabex system for Latin American and Asian developing countries, in: The Courier, Nr. 117, September-Oktober 1989, S. $94-95$.

KÖHLER, V.: Die Entwicklungspolitik der Europäischen Gemeinschaft, in: Europa-Archiv, Folge 24, 1987, S. 709 - 714.

KOMMISSION DER EG (Hrsg.): Zusammenfassender Bericht über das mit dem Abkommen von Lomé eingeführte System zur Stabilisierung der Ausfuhrerlöse in den Anwendungsjahren 1975 bis 1979, SEK (81) 1104, Brüssel 1981.

KOMMISSION DER EG (Hrsg.): Der Haushalt der Europäischen Gemeinschaft, Dritte Ausgabe, Europäische Dokumentation, 5/1981, Luxemburg 1981.

KOMMISSION DER EG (Hrsg.): Importations CEE des principaux produits en provenance des etats ACP 1976 - 1980, VIII/1182/81-FR, o.O., 3. Dezember 1981. 
KOMMISSION DER EG (Hrsg.): Memorandum zur Entwicklungspolitik der EG, Bulletin der EG 5/82, Brüssel, Mai 1982.

KOMMISSION DER EG (Hrsg.): Supplement zum Amtsblatt der EG, Nr. S 122, 30. Juni 1982, S. 3 - 7; Nr. S 226, 23. November 1982, S. $10-13$ und Nr. S 37, 23. Februar 1983, S. 3 - 5.

KOMMISSION DER EG (Hrsg.): Auswärtige Beziehungen, in: Europa-Information 63/82, Brüssel, Oktober 1982.

KOMMISSION DER EG (Hrsg.): Das EWG-AKP-System zur Stabilisierung der Ausfuhrerlöse, Informatorische Aufzeichnung, P 46, Brüssel, Mai 1983.

KOMMISSION DER EG (Hrsg.): Bericht der Kommission an den AKPEWG-Ministerrat über die finanzielle und technische Zusammenarbeit 1982, im Rahmen des Abkommens von Lomé, Kom. (83) 486 endg., Brüssel, 29. Juli 1983.

KOMMISSION DER EG (Hrsg.): Amtsblatt der EG (franz. Vers.), C 271, 26. Jg., 8. Oktober 1983.

KOMMISSION DER EG (Hrsg.): Communication de la Commission au Conseil: Les relations de la Communauté Européene et les Etats ACP dans le domaine minier, Com (83) 651 final, Brüssel, 8. November 1983.

KOMMISSION DER EG (Hrsg.): Mitteilungen an die Presse, IP (84) 347, 11. Oktober 1984.

KOMMISSION DER EG (Hrsg.): Beschlussfassungsverfahren für die besondere Finanzierungsfazilität für Bergbauerzeugnisse gemäß Titel II, Kapitel III des Dritten AKP-EWG-Abkommens, Kom (86) 183 endg., Brüssel, 10. April 1986.

KOMMISSION DER EG (Hrsg.): Die Europäische Gemeinschaft und Lateinamerika, Luxemburg, Juni - Juli 1986.

KOMMISSION DER EG (Hrsg.): Zehn Jahre Lomé, Geschichte der Partnerschaft AKP-EWG 1976 - 1985, SEK (86) 1295, Brüssel, 22. Juli 1986. 
KOMMISSION DER EG (Hrsg.): Ten years of Lome; Report on the implementation of financial and technical cooperation under the first two Lome Conventions, o.O., September 1986.

KOMMISSION DER EG (Hrsg.): Amtsblatt der EG, C 177, 30. Jg., 6. Juli 1987.

KOMMISSION DER EG (Hrsg.): Die Europäische Gemeinschaft in der Welt, Luxemburg 1988.

KOMMISSION DER EG (Hrsg.): Bericht der Kommission an den Rat über die Sondierungsgespräche mit den Mittelmeer- und den Beitrittsländern, Kom (84) 107 vom 11.05.1984, zit. in: derselbe: Europa - Dritte Welt, ein Dialog, Luxemburg 1989, S. 43.

KOMMISSION DER EG (Hrsg.): Lomé III - Mid term review 1986 1988, Brüssel 1989.

KOMMISSION DER EG (Hrsg.): Europa - Dritte Welt, ein Dialog, Luxemburg 1989.

KOMMISSION DER EG (Hrsg.): Reviewing aid to the Latin American and Asian LLDC's, in: The Courier, Nr. 117, September - Oktober 1989, S. 48 - 49.

KOMMISSION DER EG (Hrsg.): Amtsblatt der EG, Nr. L 24, 29. Januar 1990.

KOMMISSION DER EG (Hrsg.): Lomé IV 1990 - 2000, Hintergrund, Neuerungen, Verbesserungen, in: Europa Information, März 1990.

KOMMISSION DER EG (Hrsg.): Mitteilungen an die Presse, IP (90) 467, 13. Juni 1990.

KOMMISSION DER EG/EUROPÄISCHE INVESTITIONSBANK (Hrsg.): Die Finanzierung von Projekten zur Erschließung des Bergbau- und Energiepotentiales im Rahmen des 2. Abkommens von Lomé, o.O., 1982. 
KOMMISSION DER EG/EUROPÄISCHE INVESTITIONSBANK (Hrsg.): Financing of mining projects, in: The Courier, Nr. 109, Mai - Juni 1988, S. 11 - 14.

KRABBE, G.: Chiluba steht in Sambia vor einer schweren Aufgabe, in: Frankfurter Allgemeine Zeitung (FAZ), 4. November 1991

KRAPPEL, F.: Die Havanna Charta und die Entwicklung des Weltrobstoffhandels, Berlin 1975.

KRAUSE, K.P.: Die unbewältigte Zinnkrise, in: Frankfurter Allgemeine Zeitung (FAZ), 10. Februar 1986.

KRÖNER, E.: Das Stabex-System, zum Ausgleich der Exporterlösschwankungen - Darstellung, Kritik und Perspektiven, in: Dams, T.; Grohs, G.: Kontroversen in der internationalen Rohstoffpolitik, München 1977, S. 145 - 152.

KROHN, H.B.: Das Abkommen von Lomé (I) zwischen der EG und den AKP-Staaten, in: Europa-Archiv, Folge 6, 1975, S. 177 - 188.

KRÜGER, J., et al.: Kupfer, in: Gocht, W. (Hrsg.): Handbuch der Metallmärkte, Berlin, Heidelberg, New York 1985, S. 177 - 215.

KÜRSTEN, M.: Geographische Verteilung und Rohstofforschung - Elemente der Rohstoffsicherung, in: Vortragsveröffentlichungen Haus der Technik, Heft 439, Essen 1981, S. 3 - 8.

LAIDLAW, K.: The Common Fund: a major breakthrough?, in: ICDAnews, UNCTAD Bulletin, 2. April 1983, S. 2.

LECHNER, H.; SAMES, C.W.; WELLMER, F.W.: Mineralische Rohstoffe im Wandel, Essen 1987.

LINGNAU, H.: Neue Elemente in der Entwicklungszusammenarbeit der Europäischen Gemeinschaft zu Beginn der 90er Jahre, Deutsches Institut für Entwicklungspolitik, Berlin, Mai 1991.

LOFTUS; J.: Cooperation between Papua New Guinea and the European Community, in: The Courier, Nr. 133, Mai - Juni 1992, S. 37 - 40. 
LÜTTIG, G.: Die Entwicklungsländer mit geringem Geopotential, Hannover 1978.

MAENNIG, W.: Zur Kritik des EG-Sonderfonds für Bergbauerzeugnisse (SYSMIN), in: Metall, 41. Jahrgang, Heft 10, Oktober 1987, S. $1042-1046$.

MAILLET, P.: Die Wirtschaft der Europäischen Gemeinschaft, Europäische Dokumentation, 1-2/1982, Luxemburg 1982.

MARIN, M.: Lomé IV - scope of a new convention, in: The Courier, Nr. 120, März - April 1990, S. 12 - 13.

MARTIN, A.: ACP-EEC cooperation in mining, energy and investment, in: The Courier, Nr. 73, Mai - Juni 1982, S. 24 - 26.

McQUEEN, M.: Lomé and industrial co-operation, in: Journal of World Trade Law, 17. Jg., Nr. 6, Dezember 1983, S. 524 - 532.

MEGZARI, A.: The Common Fund for commodities, in: The Courier, Nc. 116, Juli - August 1989, S. $72-75$.

METALLGESELLSCHAFT (Hrsg.): Metalistatistik 1975/1985, Frankfurt am Main 1986; 1979/1989, Frankfurt am Main 1990.

METALS \& MINERALS ANNUAL REVIEW 1990.

MEYER, K.: Die zweite Konvention von Lomé, in: Europa-Archiv, Folge 1, 1980, S. $11-20$.

MICHAELIS, H.: Memorandum über eine Europäische Rohstoffversorgungspolitik, Gutachten im Auftrag der Kommission der EG, o.O., September 1972.

MINING ANNUAL REVIEW: Jahrgänge 1976 bis 1989.

MÜLLER-OHLSEN, L.: Die Weltmetallwirtschaft im industriellen Entwicklungsprozeß, Kieler Studien 165, Tübingen 1981.

NUSCHELER, F.: Lern- und Arbeitsbuch Entwicklungspolitik, 3. Aufl., Bonn 1991. 
OCHEL, W.: Die Entwicklungsländer in der Weltwirtschaft, Köln 1982.

ORGANISATION FÜR WIRTSCHAFTLICHE ZUSAMMENARBEIT UND ENTWICKLUNG (Hrsg.): Zusammenarbeit im Dienst der Entwicklung, Bericht 1988 (Berichterstatter J.C. Wheeler), Paris 1988.

O.V.: Abkommen AKP-EWG von Lomé (I), in: Europa-Archiv, Dokumente, Folge 6, 1975, S. D 164 - 170.

O.V.: Das neue Lomé-Abkommen (II), in: Europa-Archiv, Dokumente, Folge 21, 1979, S. D 578 - 588.

O.V.: Profiles of the ACP and EEC countries, in: The Courier, Nr. 58, November 1979, S. 40 55.

O.V.: European Investment Bank, Breakdown of 1981 investments in the ACP, in: The Courier, Nr. 73, Mai - Juni 1982, S. 27 - 28.

O.V.: First Sysmin operation, ECU 55 mio. for Zambia, in: The Courier, Nr. 73, Mai - Juni 1982, S. gelb I.

O.V.: Stabex, Financing decisions for 1981, in: The Courier, Nr. 76 , November - Dezember 1982, S. gelb I - II.

O.V.: Zambia, in: Africa Research Bulletin, 15. Januar - 14. Februar 1983, S. $6742-6745$.

O.V.: Bonn will die Zusammenarbeit mit den Lomé-Entwicklungsländern fördern, in: BfA/NfA, 22. Februar 1983.

O.V.: Shaba and its copper, the "strong-box" of Zaire, in: The Courier, Nr. 78, März - April 1983, S. 44 - 46.

O.V.: Cobalt: back from the brink, in: Mining journal, Vol. 300, Nr. 7707, 6. Mai 1983, S. $301-302$.

O.V.: EEC-ACP trade, statistical analysis 1970 - 1981, in: The Courier, Nr. 79, Mai - Juni 1983, S. 57 - 60. 
0.V.: ACP memorandum on the Stabex system, in: The Courier, Nr. 79, Mai - Juni 1983, S. 83 - 84.

0.V.: AKP-Länder verlangen besseres NE-System, VWD/NfA, 20. September 1983.

O.V.: Economy struggles as copper stays down, in: Africa Research Bulletin, Oktober - November 1983, S. 7045 - 7048.

0.V.: EEC proposes ACP minex programme, in: Mining journal, Vol. 22, 2. Dezember 1983, S. 401.

O.V.: Negotiations update, in: The Courier, Nr. 85, Mai - Juni 1984, S. gelb I - III.

O.V.: Chiles Produktionskosten konkurrenzlos günstig, in: Handelsblatt, 30. August 1984.

O.V.: Larosiere: Es gibt kein Patentrezept für die Schuldenkrise, in: Frankfurter Allgemeine Zeitung (FAZ), 25. September 1984.

O.V.: Die EG-Agrarausgaben im vergangenen Jahr, in: Neue Zürcher Zeitung, 10. Oktober 1984.

O.V.: Die merkwürdige Rohstoff-Baisse, in: Frankfurter Allgemeine Zeitung (FAZ), 10. Oktober 1984.

O.V.: GNP of ACP-Countries (1981), in: The Courier, Nr. 88, November - Dezember 1984, S. 57.

O.V.: Rebuilding Zimbabwe: at 5 years of independence, ZANU, Department of the Commissariat and Culture, Harare, o.J., (1985).

O.V.: Copper - A Third World View, in: Mining journal, Vol. 304, 17. Mai 1985, S. 341 - 343.

O.V.: Ausschreibungen, in: Nachrichten für den Außenhandel, 17. Oktober 1985, S. 7 .

O.V.: Dissolution de la Somirwa, in: Marchés tropicaux, 8. November 1985, S. 2773. 
O.V.: Mühsame Sanienung der Wirtschaft Sambias, in: Blick durch die Wirtschaft, 30. April 1986.

O.V.: VIth EDF: Division of contributions, in: The Courier, Nr. 97, Mai - Juni 1986, S.V.

O.V.: Bedeutendster Abnehmer ist der Fahrzeugbau, in: Handelsblatt, 12. August 1986.

O.V.: Stabex, in: The Courier, Nr. 100, November - Dezember 1986, S. III.

O.V.: An IMF allible Leader, in: The Economist, 10. Januar 1987.

O.V.: Copper, Short-term shine, in: The Economist, 9. Mai 1987, S. 69.

O.V.: Zambia; New economic policies aim to break vicious circle, in: Africa Economic Digest, 10. Juli 1987.

O.V. Stabex, in: The Courier, Nr. 105, September - Oktober 1987, S. IX.

O.V.: Sambias Alleingang ohne viel Zukunft, in: Neue Zürcher Zeitung, 28. Januar 1988.

O.V.: Desolate Verhältnisse in Sambia, in: Neue Zürcher Zeitung, 13. Februar 1988.

O.V.: Der Kupfergürtel Lusakas verliert schnell an Glanz, in: Handelsblatt, 23. Februar 1988.

O.V.: Afrika sucht nach einem Ausweg aus der Krise, in: Blick durch die Wirtschaft, 26. April 1988.

O.V.: Simbabwe: Der Bergbau mit einem besseren Ergebnis, in: Blick durch die Wirtschaft, 9. Mai 1988.

O.V.: Financing of mining projects, in: The Courier, Nr. 109, Mai - Juni 1988, S. 13. 
O.V.: Sysmin: Niger, research into coal and gold mining, in: The Courier, Nr. 111, September - Oktober 1988, S. XI.

O.V.: Bauxit-Darlehen für Guayana, in: BfA/NfA, 15. November 1988.

O.V.: Aluminium, in: Financial Times, Section IV, 26. Oktober 1988, S. I - IV.

O.V.: Stabex, in: The Courier, Nr. 112, November - Dezember 1988, S. XV.

O.V.: Aufschwung auf schwacher Basis in Simbabwe, in: Neue Zürcher Zeitung, 7. Dezember 1988.

O.V.: Sysmin, in: The Courier, Nr. 113, Januar - Februar 1989, S. XI - XII.

O.V.: Italien und Griechenland blockieren die Verhandlungen mit den AKP-Staaten, in: Handelsblatt, 19. Juli 1989.

O.V.: Sysmin, Botswana, in: The Courier, Nr. 117, September - Oktober 1989, S. IV gelb f.

O.V.: Stabex: Teillösung für das Defizit im Anwendungsjahr 1988, in: Europa Entwicklung, Oktober 1989, Brüssel, S. 4

O.V.: Zahl der AKP-Staaten soll erhöht werden, in: Frankfurter Allgemeine Zeitung (FAZ), 29. November 1989.

O.V.: AKP mit EG über Hilfe einig, in: Blick durch die Wirtschaft, 7. Dezember 1989.

O.V.: Die Minenkrise stürzt die Wirtschaft in Ungewissheit, in: Handelsblatt, 2. Januar 1990.

O.V.: Sysmin - Phosphate mining industry, in: The Courier, Nr. 119, Januar - Februar 1990, S. II gelb.

O.V.: Simbabwe will den Bergbau intensivieren, in: Blick durch die Wirtschaft, 12. Februar 1990. 
O.V.: Mit zahlreichen Programmen will die EG Firmen beider Regionen zusammenführen, in: Handelsblatt, 27. Februar 1990.

O.V.: Details on principal developments and innovations, in: The Courier, Nr. 120, März - April 1990, S. 14 - 16.

O.V.: Lome-IV-Abkommen weitgehend in Kraft, in: Commerzbank, AuBenhandelsblätter 4/90, S. 7 - 8.

O.V.: Kupfer: Vorrãte reichen langfristig zur Deckung des Verbrauchs, in: Handelsblatt, 5. Juli 1990.

O.V.: Umfangreiche EG-Hilfe für Osteuropa, in: Blick durch die Wirtschaft, 12. Dezember 1990.

O.V.: Sysmin, Senegal, in: The Courier, Nr. 125, Januar - Februar 1991, S. IV gelb.

O.V.: Sysmin, ECU 15000000 to help Senegal solve the problem of cadmium, in: The Courier, Nr. 126, März - April 1991, S. IX gelb.

O.V.: Europa-Bank wird eröffnet, in: Frankfurter Aligemeine Zeitung (FAZ), 13. April 1991.

O.V.: Die Aluminium-Industrie rechnet mit einem hohen Materialrücklauf, in: Blick durch die Wirtschaft, 15. April 1991.

O.V.: Gesetz zu dem Vierten AKP-EWG-Abkommen von Lomé vom 15. Dezember 1989 sowie zu den mit diesem Abkommen in Zusammenhang stehenden Abkommen vom 17. Dezember 1990, in: Bundesgesetzblatt Teil II, Z 1998 A, Ausgegeben zu Bonn am 12. Januar 1991

O.V.: Europas Pflichten für den Rest der Welt, in: Europa ohne Grenzen, Alarm für die Umwelt, Spiegel Spezial, 1/1992, S. 150 - 162.

O.V.: Möglichkeiten für europäische Unternehmen im Rahmen von Lome, in: Blick durch die Wirtschaft, 8. Januar 1992.

O.V.: Sysmin, adapting to Lome IV, in: The Courier, Nr. 131, Januar Februar 1992, S. gelb VII. 
O.V.: Ressourcen-Optimierung, in: Handelsblatt, 15. Juni 1992.

O.V.: Verstärkte Kooperation für den Technologietransfer, in: Handelsblatt, 15. Juni 1992.

O.V.: Convention at work, in: The Courier, Nr. 137, Januar - Februar 1993, S. II gelb.

O.V.: Convention at work, in: The Courier, Nr. 139, Mai - Juni 1993, S. I gelb.

OYOWE, A.: A convention for a changing world, in: The Courier, Nr. 120, März - April 1990, S. 2 - 4.

PAGNI, L.: Getting out of the copper straitjacket, in: The Courier, Nr. 85, Mai - Juni 1984, S. 18 - 24.

POMMERENING, G., et al.: Möglichkeiten zur Weiterverarbeitung ausgewählter NE-Metallerze in Entwicklungsländern, ite, Hamburg, Februar 1977.

PRESSE- UND INFORMATIONSBLATT DER BUNDESREGIERUNG (Hrsg.): Aktuelle Beiträge zur Wirtschafts- und Finanzpolitik, Nr. 2/1987: Versorgungslage bei Rohstoffen, Kurzfassung einer Untersuchung im Auftrage des BMWi, Bonn, 8. Januar 1987.

RAJANA, C.: Europe undermined: the Lome response; an evaluation of the EEC-ACP non-fuel-minerals arrangement, in: Africa Development, Vol. VI, Nr. 2, April - Juni 1982, S. 5 - 36.

RECHNUNGSHOF DER EG (Hrsg.): Jahresbericht zum Haushaltsjahr 1985, in: Amtsblatt der EG, C 321, 29. Jg., 15. Dezember 1986.

ROBBINS, P.; EDWARDS, J.: Guide to Non-Ferrous Metals and their markets, 2. Aufl., London 1980.

ROSTOW, W.,W.: Stadien wirtschaftlichen Wachstums. Eine Alternative zur marxistischen Entwicklungstheorie, Göttingen 1960.

SAAGER, R.: Metallische Rohstoffe von Antimon bis Zirkonium, Bank von Tobel (Hrsg.), Zürich 1984. 
SAMES, C.W.: Welt im Rohstoffüberfluß, in: Metall, 38. Jg., Heft 4, April 1984, S. 325 - 327.

SAMES, C.W.: Anaconda, Berichte aus der Rohstoffwelt, München 1986.

SCHIPULLE, H.: Die Entwicklungspolitik der Europäischen Gemeinschaft, (Brüssel) 1981.

SCHIRMER, W.; MEYER-WÖBSE, G.: Internationale Rohstoffabkommen, München 1980.

SCHMIDT, H.; KRUSZONA, M.: Regionale Verteilung der Weltbergbauproduktion und der Weltvorräte mineralischer Rohstoffe, Bundesanstalt für Geowissenschaften und Rohstoffe (Hrsg.), Hannover 1982.

SCHMUCK, O.: Lomé - Zusammenarbeit auf neuer Grundlage, in: Entwicklung und Zusammenarbeit, 3/90, S. 16 - 17.

SCHÜTZDELLER, R.: Sekundäreffekte von Bergbauprojekten, Diss. RWTH Aachen, Internationale Kooperation, Bd. 25, Baden-Baden 1984.

SEFALI, M.: A significant contribution to human understanding and development, in: The Courier, Nr. 120, März - April 1990, S. 6 - 8.

SHARMA, Y.: Welche Zukunft für Stabex, in: Entwicklung und Zusammenarbeit, Vol. 24, November 1983, S. 6 - 7.

SMITH, M.: ZCCM on course for recovery, in: Mining Magazine, Dezember 1987, S. 524 - 535.

SPRENG, D.T.: Tiefgreifender Strukturwandel in der weltweiten Aluminiumindustrie, in: Neue Zürcher Zeitung, 1. August 1981.

STATISTISCHES AMT DER EG (Hrsg.): Statistique de Base de la Communaute 1981, Luxemburg 1982. 
STATISTISCHES AMT DER EG (Hrsg.): Beilagen zum monatlichen Außenhandelsbulletin, Luxemburg, zit. in: Fuchs, G.: Bericht im Namen des paritätischen Ausschusses, Teil Anhang, Beratende Versammlung AKP-EWG Sitzungsdokumente 1983 - 1984, 30. August 1983.

STATISTISCHES AMT DER EG (Hrsg.): EG-Rohstoffbilanzen, 1980 und 1981, Luxemburg 1982, 1983.

STATISTISCHES AMT DER EG (Hrsg.): EC-ACP-Trade, a statistical analysis 1970 - 1981, Luxemburg 1983.

STATISTISCHES AMT DER EG (Hrsg.): EG-Rohstoffbilanzen 1979 1982, Luxemburg 1985.

STATISTISCHES AMT DER EG (Hrsg.): Rohstoffe, Versorgung der EG, 1975 - 1983, Luxemburg 1986.

STATISTISCHES AMT DER EG (Hrsg.): Foreign trade: third countries, statistical yearbook 1977 - 1983, Vol. A, ACP-countries, Luxemburg 1986.

STATISTISCHES AMT DER EG (Hrsg.): Analytische Übersichten des Außenhandels, NIMEXE, Import 1981, 1985, Band B, H, I, Z, Luxemburg 1983, 1986.

STATISTISCHES AMT DER EG (Hrsg.): ACP basic statistics 1986, Luxemburg 1986.

STATISTISCHES AMT DER EG (Hrsg.): EEC-ACP trade pattern: greater expansion in 1985, in: The Courier, Nr. 97, Mai - Juni 1986, S. $58-59$.

STATISTISCHES AMT DER EG (Hrsg.): Key figures, Supplement Target 92, No 6-1992.

STATISTISCHES BUNDESAMT WIESBADEN (Hrsg.): Statistik des Auslandes, Länder(kurz)bericht, Surinam 1987, Zaire 1990, Guayana 1993, Mauretanien 1991, Pazifische Staaten 1986, Sambia 1983 und 1991, Simbabwe 1983 und 1990, Stuttgart $1986-1993$. 
STEVENS, C.: Die Lehren aus Lomé III, in: epd - Entwicklungspolitik: Vorausinformation 7/88, Frankfurt/M. 1988.

STEVENS, C.: Commodity prices in the 1980s: a decade of decline for the ACPs, in: The Courier, Nr. 116, Juli - August 1989, S. $60-62$.

ST. JOHN, B.: "The groundwork of our continued cooperation in the 1980s", in: The Courier, Nr. 58, November 1979, S. 4 - 6.

STRIZEK, H.: Die neue AKP-EWG-Konvention (Lomé II), in: Entwicklungspolitik, Materialien Nr. 66: Lomé II, Bundesministerium für wirtschaftliche Zusammenarbeit (Hrsg.), Bonn 1980, S. 6 - 10.

SULONG, W.: Tin gloom lifts in Malaysia, in: Financial Times, 31. Oktober 1986.

TEZLAFF, R.: Lomé II: "Nachgeschmack von Frustration", in: epd-Entwicklungspolitik 4/80, S. 9-14.

THE ECONOMIST INTELLIGENCE UNIT (Hrsg.): Country Report: Zambia, London, April 1987.

TIETZEL, M.: Internationale Rohstoffpolitik, Bonn 1977.

TWITCHETT, C.C.: Lomé II: die Enttäuschungen der AKP-Länder, in: Europa-Archiv, Folge 3, 1980, S. 81 - 88.

UNCTAD (Hrsg.): Les systemes Stabex et Sysmin, TD/B/C.1/237, Genf, 26. Januar 1983.

UNCTAD (Hrsg.): Compensatory financing of export earnings shortfalls, New York 1986.

VERBERT, M.: The Stabex system for Latin America and Asian developing countries, in: The Courier, Nr. 117, September - Oktober 1989, S. 94 - 95 .

VEREINTE NATIONEN (Hrsg.): Commitee on Natural Resources, New York 1979, zit. in: Gocht, W.: Wirtschaftsgeologie und Rohstoffpolitik, 2. Aufl., Berlin, Heidelberg, New York, Tokyo 1983, S. 279. 
VERGEER, U.: Bericht im Namen des Paritätischen Ausschusses, Teil A: Entschließungsantrag, Teil B: Begründung, Beratende Versammlung AKP-EWG Sitzungsdokumente 1982 - 1983, 3. November 1982.

WAGNER, W., et al.: Die wirtschaftliche und rohstoffpolitische Bedeutung Afrikas und seiner Regionen für die Bundesrepublik Deutschland, ite, Hamburg, Januar 1981.

WAGSTYL, S.: Innovations in mine financing, in: Financial Times, 15. August 1986.

WALTHER, H.W., et al.: Eisen, in: Gocht, W. (Hrsg.): Handbuch der Metallmärkte, Berlin 1985, S. 30 - 69.

WAWRZIK, K.: Bericht im Namen des Ausschusses für Entwicklung und Zusammenarbeit, Europa-Parlament, Sitzungsdokumente 1980 1981, 17. November 1980.

WAWRZIK, K.: Handel und Märkte, Unterbericht zum Bericht im Namen des Ausschusses für Entwicklung und Zusammenarbeit, EuropaParlament, Sitzungsdokumente 1983 - 1984, 12. August 1983.

WELTBANK (Hrsg.): World Development Report 1983, Washington, D.C. (1983).

WELTBANK (Hrsg.): Weltentwicklungsbericht 1984, Washington, D.C. (1984).

WELTBANK (Hrsg.): Jahresbericht 1985, Washington, D.C. (1985).

WELTBANK (Hrsg.): Price prospects for major primary commodities, Vol. IV: Metals and Minerals, Washington, D.C., Oktober 1986.

WELTBANK (Hrsg.): The World Bank Atlas, Washington, D.C., December 1992

WILKINSON, M.: Tin price climbs back above GBP 4000 a tonne, in: Financial Tímes, 22. Oktober 1986.

YPERSELE, J.; KOEUNE, J.C.: Das Europäische Währungssystem, Luxemburg 1985. 
ZAMBIA MINING YEARBOOK 1980, Kitwe, o.J.

ZAMBIA CONSOLIDATED COPPER MINES (Hrsg.): Annual Report 1982, 1983, 1986, 1987, 1988, Lusaka $(1982,1983,1986,1987,1988)$. 


\section{$u b$ \\ RWTHAMCHEI

Diese Seite enthält personenbezogene Daten und wurde daher nicht digitalisiert. 
$s 212156=2$

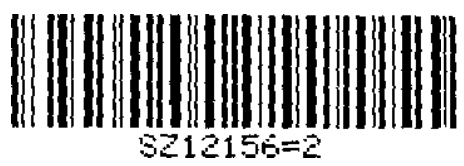


$\Delta$

$4 \pi$

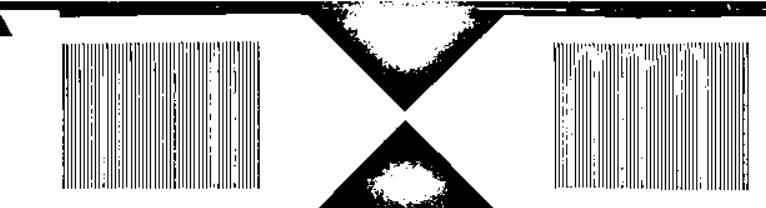

$\Delta$

$\underline{\underline{a}}$ 으 in

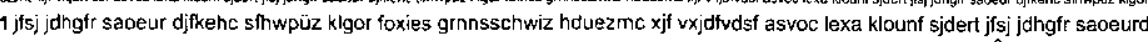

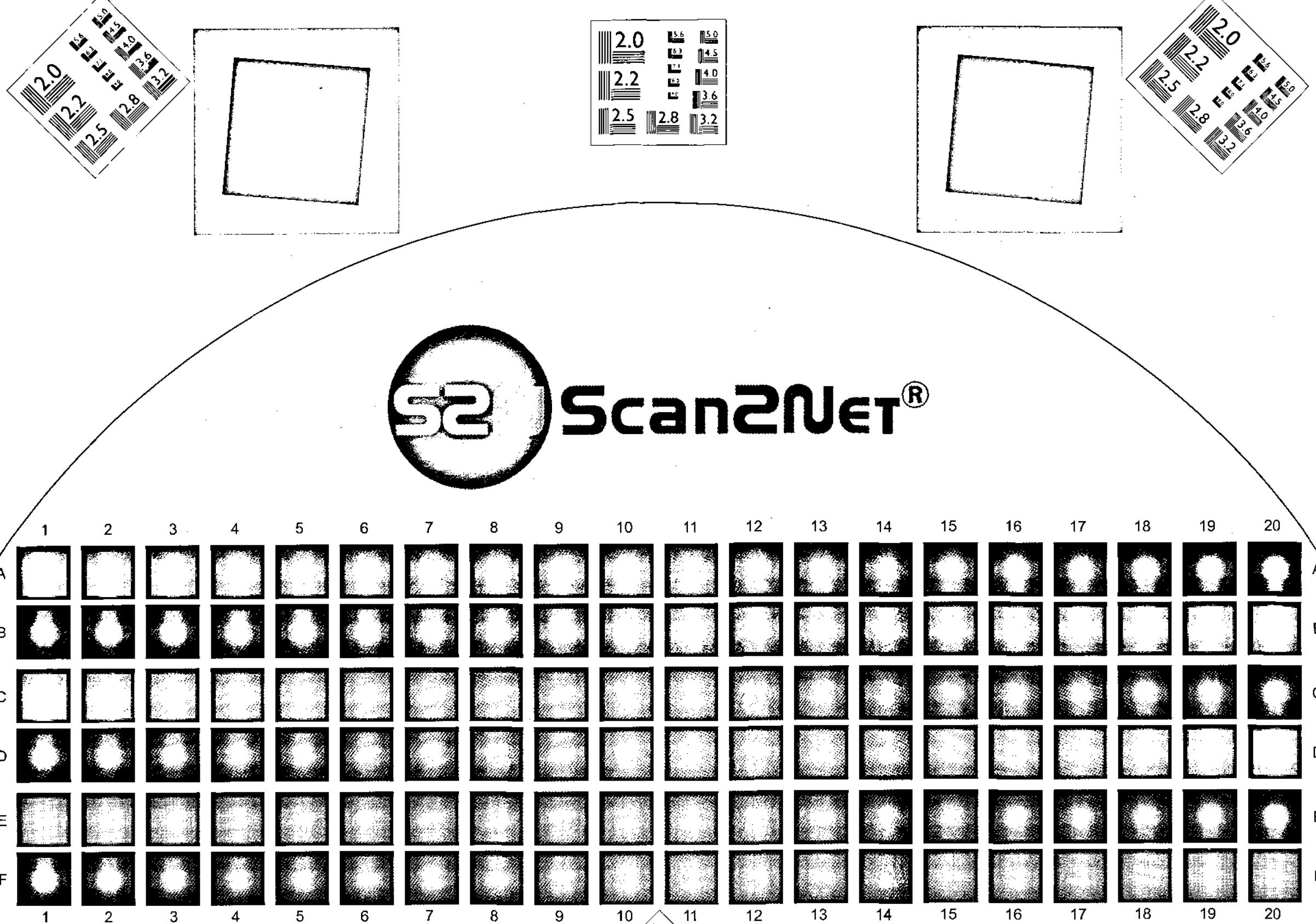

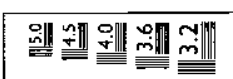

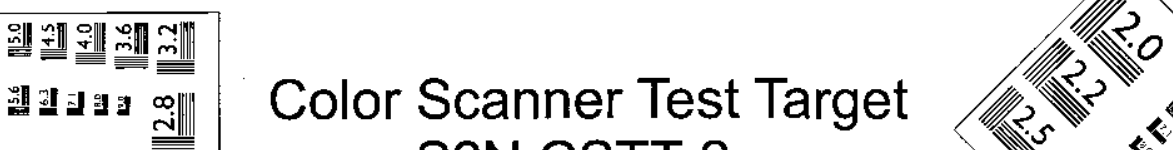

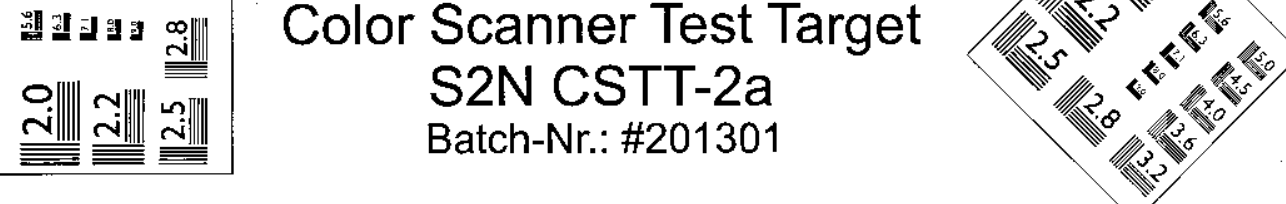

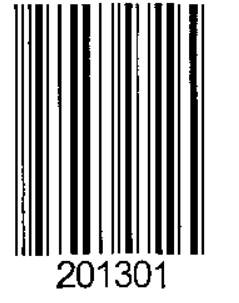

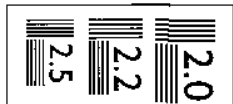

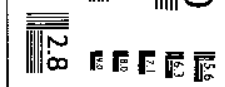

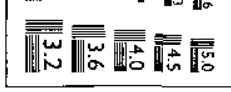

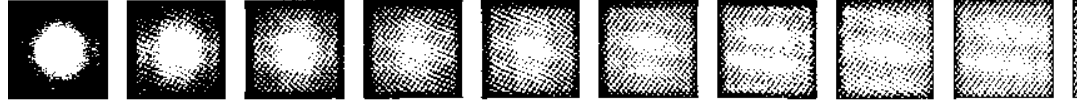

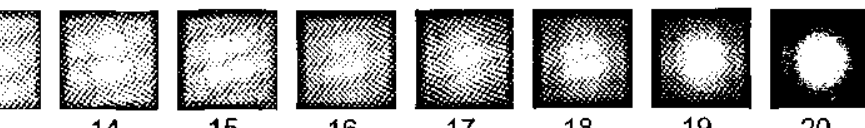

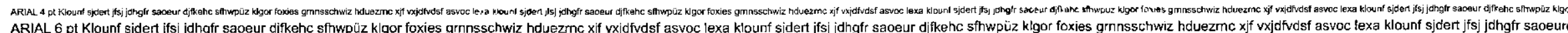

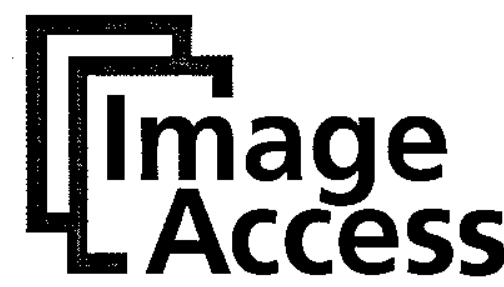

Hatzfelder Strasse $161-163$

D-42281 Wuppertal

Telefon: $++49(0) 20227058-0$

Telefax: ++49 (0)202 27058-40

www.imageaccess.de

www.scan2net.de

E-Mail: info@imageaccess.de

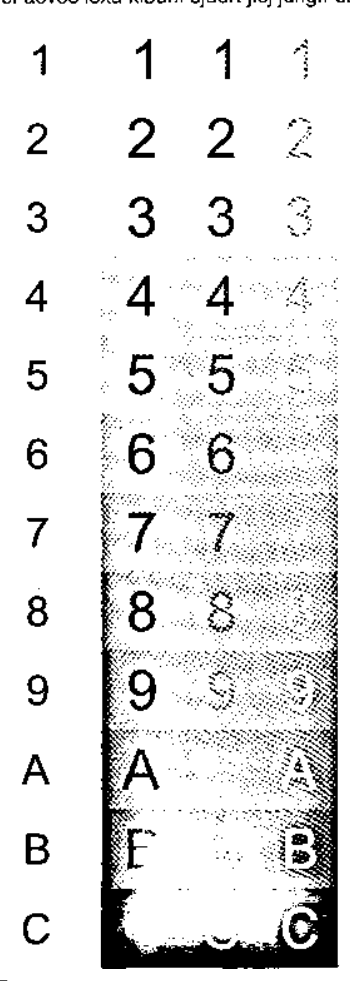

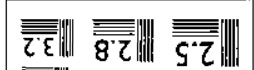

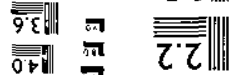

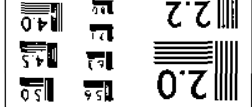

$4 \mathrm{P}$

Klounf sjdert jisj jothgir saoeur djikehc sthwpüz kigor foxies grnn
Wiu liffc jmv prongs wallusi 94846393 shd94 hah 43 gnurz ong

Klounf sjdert jfsj jdhgfr saoeur djfkehc sfhwpüzk

Wju licfc jmv prongs wallusi 94846393 shd94

10D Klounf sjdert jfsj jdhgfr saoeur djfkehc Wju licfc jmv prongs wallusi 9484639

12p Klounf sjdert jfsj jdhgfr saoeur d Wju licfc jmv prongs wallusi 948

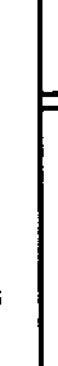

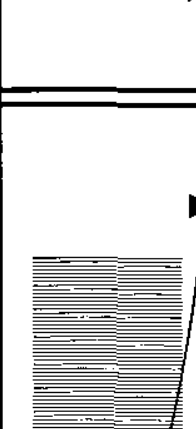

$\langle>$

-
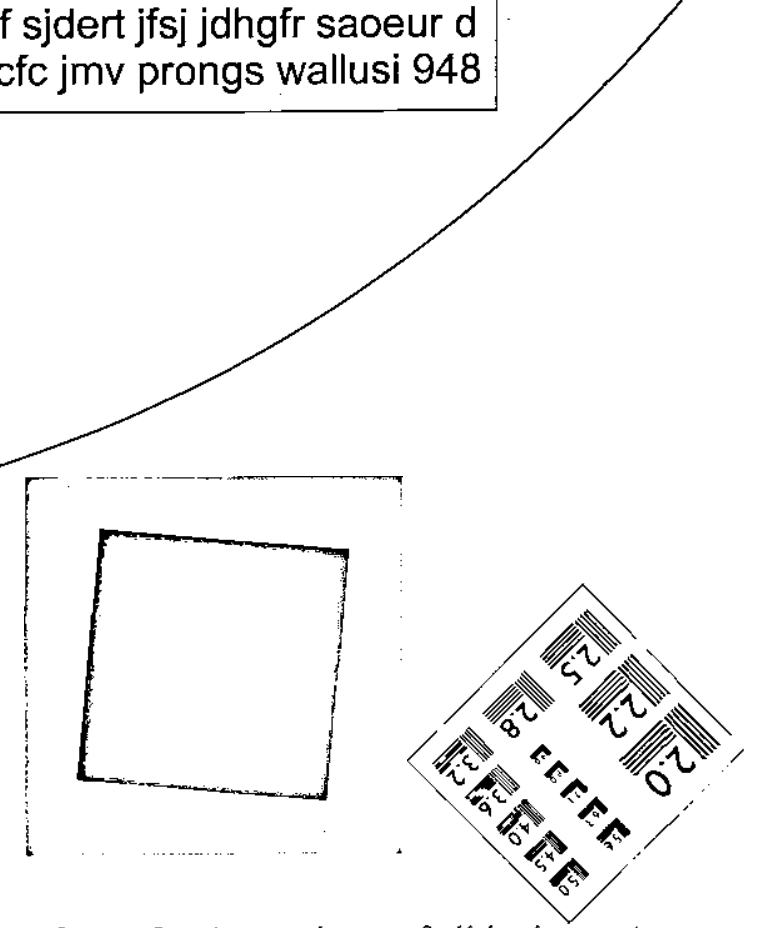

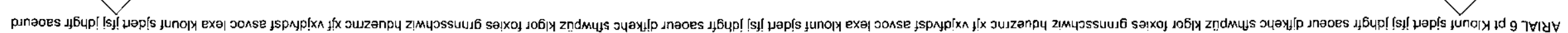
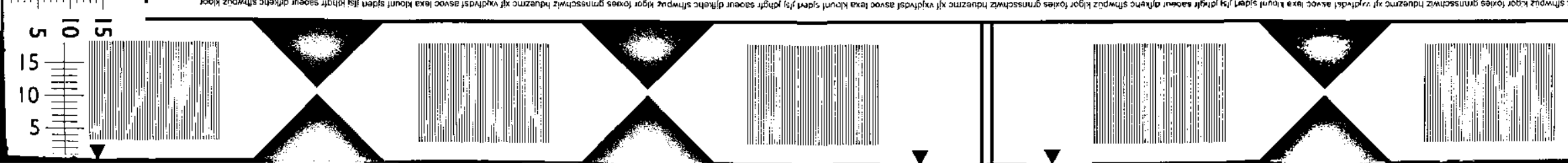